DOE/RA/33210-01

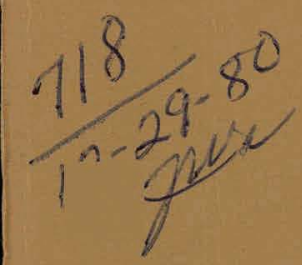

Prepared for: 2

U.S. Department of Energy

Assistant Secretary for

Resource Applications

Office of Oil and Natural Gas

Under Contract No. DE-AC01-79RA-33210

\title{
Abandoned \\ Texas Oil Fields
}

MASTER

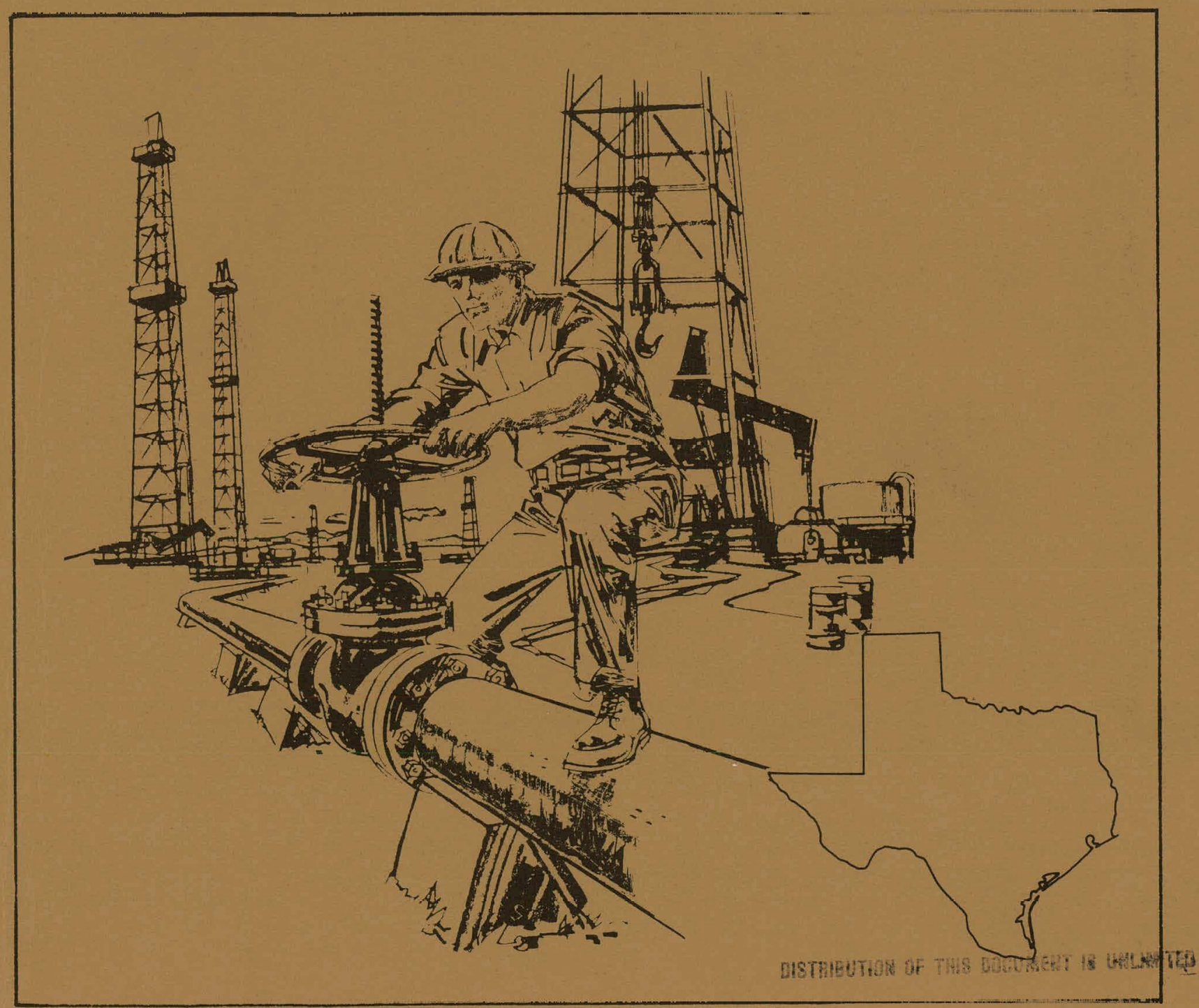




\section{DISCLAIMER}

This report was prepared as an account of work sponsored by an agency of the United States Government. Neither the United States Government nor any agency Thereof, nor any of their employees, makes any warranty, express or implied, or assumes any legal liability or responsibility for the accuracy, completeness, or usefulness of any information, apparatus, product, or process disclosed, or represents that its use would not infringe privately owned rights. Reference herein to any specific commercial product, process, or service by trade name, trademark, manufacturer, or otherwise does not necessarily constitute or imply its endorsement, recommendation, or favoring by the United States Government or any agency thereof. The views and opinions of authors expressed herein do not necessarily state or reflect those of the United States Government or any agency thereof. 


\section{DISCLAIMER}

Portions of this document may be illegible in electronic image products. Images are produced from the best available original document. 


\section{NOTICE}

This report was prepared as an account of work sponsored by the United States Government. Neither the United States nor the United States Department of Energy, nor any of their employees, makes any warranty, express or implied, or assumes any legal liability or responsibility for the accuracy, completeness, or usefulness of any information, apparatus, product, or process disclosed, or represents that its use would not infringe privately owned rights. Reference herein to any specific commercial product, process, or service by trade name, mark, manufacturer, or otherwise, does not necessarily constitute or imply its endorsement, recommendation, or favoring by the United States Government or any agency thereof. The views and opinions of authors expressed herein do not necessarily state or reflect those of the United States Government or any agency thereof.

Ava1lable from:

National Technical Information Service (NIIS)

ర.S. Department of Commerce

5285 Port Royal Road

Spriagfield, Virginia 22161

Price: Priated copy: $\$ 24.50$

Microfiche: $\$ 3.50$ 
DOE/RA/33210-01

Dist. Category UC92a

\section{Abandoned Texas Oil Fields}

December 1980

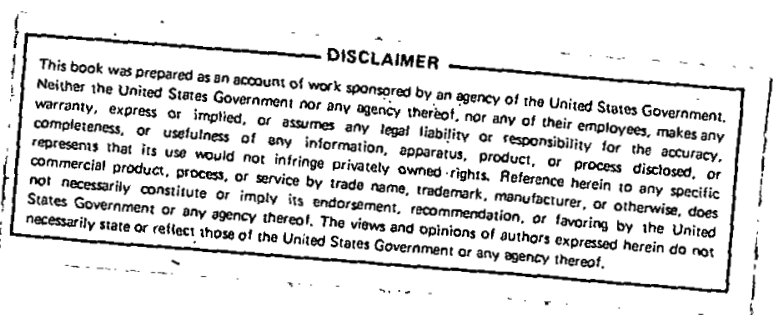

Prepared by:

Boston Technologies Inc.

Cambridge, Maryland 02139

Under Contract No. DE-AC01-79RA-33210

Prepared for:

U.S. Department of Energy

Assistant Secretary for Resource Applications

Office of Oil and Natural Gas

Washington,D.C. 20461

DISTRIBUTION OF THS DOSUAERT IS UALSHTE 
OVERVIEW

The Office of $0 i l$ and Natural Gas, Resource Applications, is charged with sponsoring the commercial development of additional domestic oil and gas supplies. As part of this effort, the office is investigating the potential of recovering additional oil and gas from abandoned oil fields.

A preliminary study was conducted of abandoned oil fields in Texas to:

- Assess the potential resource from abandoned fields

0 Assess the barriers to reactivation of the abandoned fields

- Provide an easily accessible and useful data source to be used as a preliminary screen for identifying fields for further technical and investment evaluations.

This study indicates that a considerable amount of petroleum may be economically recoverable at today's oil prices from reservoirs that were abandoned when prevailing prices were lower. In addition, reservoirs that were never tested or were tested and determined to be uneconomic may now prove to be economical to produce.

A major barrier to the timely production from the abandoned fields has been the lack of pertinent, readily available, and systemized information. Producers have, for some time, learned of previous exploration and production efforts in an area and gone to the State regulatory agency to determine if they might find a good prospect. Drawing on several sources, the DOE has developed a listing of believed unduplicated data in one source. The DOE hopes the general availability of these data will catalyze the private sector recovery of this unproduced resource. 
DATA SOURCES

Data for Texas abandoned oil fields were primarily derived from two sources:

1) Texas Railroad Commission (TRRC), and

2) Dwight's ENERGYDATA.

For purposes of this report, abandoned oil fields are defined as those fields that had no production during 1977. Following the definition used by the Texas State regulatory agency, the Texas Railraod Commission, a "field" is defined as an individual reservoir.

$\therefore$ The TRRC OILMASTER computer tapes were used to identify these abandoned oil fields. The tapes also provided data on formation depth, gravity of $0 i 1$ production, location (both district and county), discovery date, and the cumulative production of the field since its discovery.

In all, the computer tapes identified 9,211 abandoned fields, most of which had less than 250,000 barrel cumulative production. This report focuses on the 676 abandoned onshore Texas oil fields that had cumulative production of over 250,000 barrels.

The Dwight's ENERGYDATA computer tapes provided production histories for approximately two-thirds of the larger fields abandoned in 1966 and thereafter. Fields which ceased production prior to 1966 will show no production history nor abandonment date in this report.

\section{REPORT FORMAT}

Figure I presents a sample page of the data produced with numbered data elements keyed to those listed below: 
(1) Texas Railroad Commission District

(2) Discovery date of field (reservoir)

(3) Year of last production, if known

(4) Field number, as designated by TRRC

(5) Field name

(6) Depth of field (in feet)

(7) Gravity of oil production (in degrees API)

(8) Cumulative production of field since discovery (in 42 gal. barrels)

(9)... County (where fields are located under more than one county only one county is listed)

Data elements 10 though 14 are provided when available.

(10) Calendar year

(11) Average number of flowing wells producing during year

(12) Average number of artificial lift wells producing during year

(13) Yearly field oil production

(14) Average daity oil output per well, measured in barrels per well-day

(15) Yearly field gas production

Data elements 16 through 18 provide summations at the county, district, and State level.

(16) Number of fields and cumulative production of abandoned fields in designated county

(17) Number of fields and cumulative production of abandoned fields in designated TRRC district

(18) Number of fields and cumulative production of the 676 abandoned fields listed. 


\section{Figure 1}

(1) aBANDONED ONSHORE O - I FIELDS WITH OVE
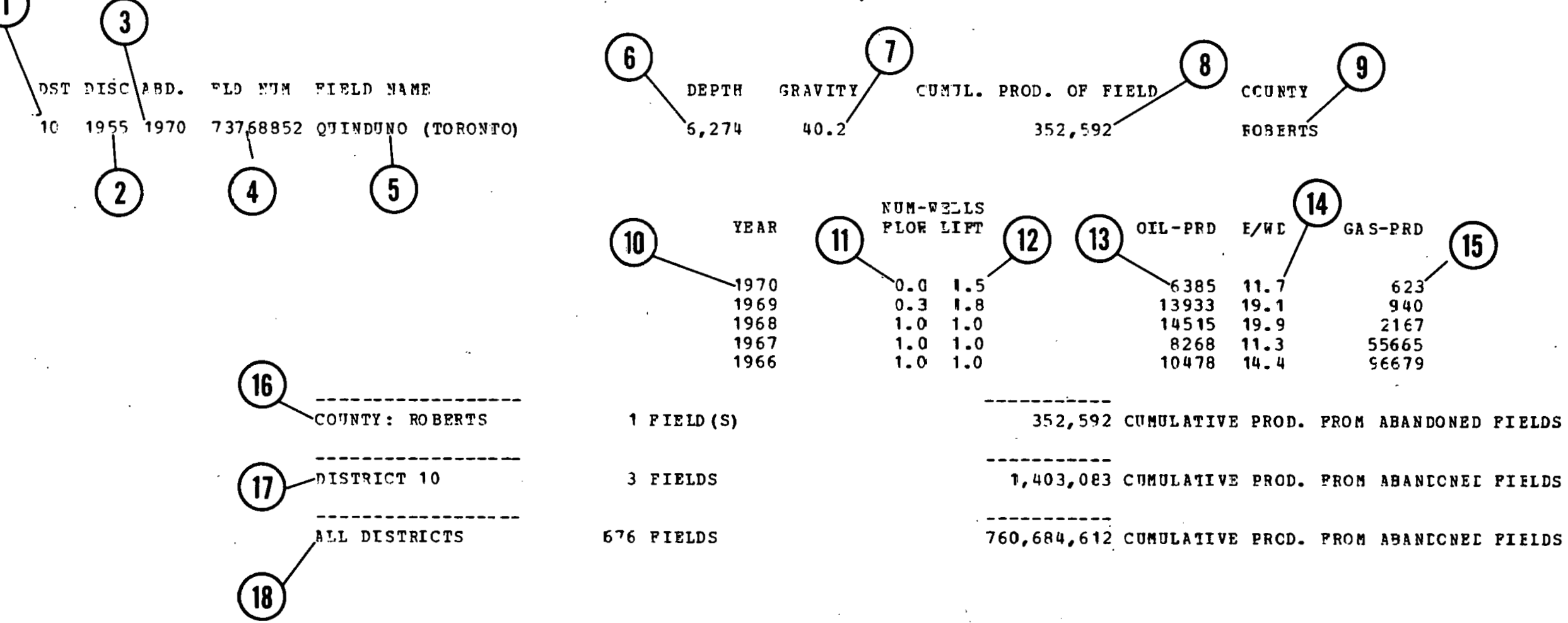


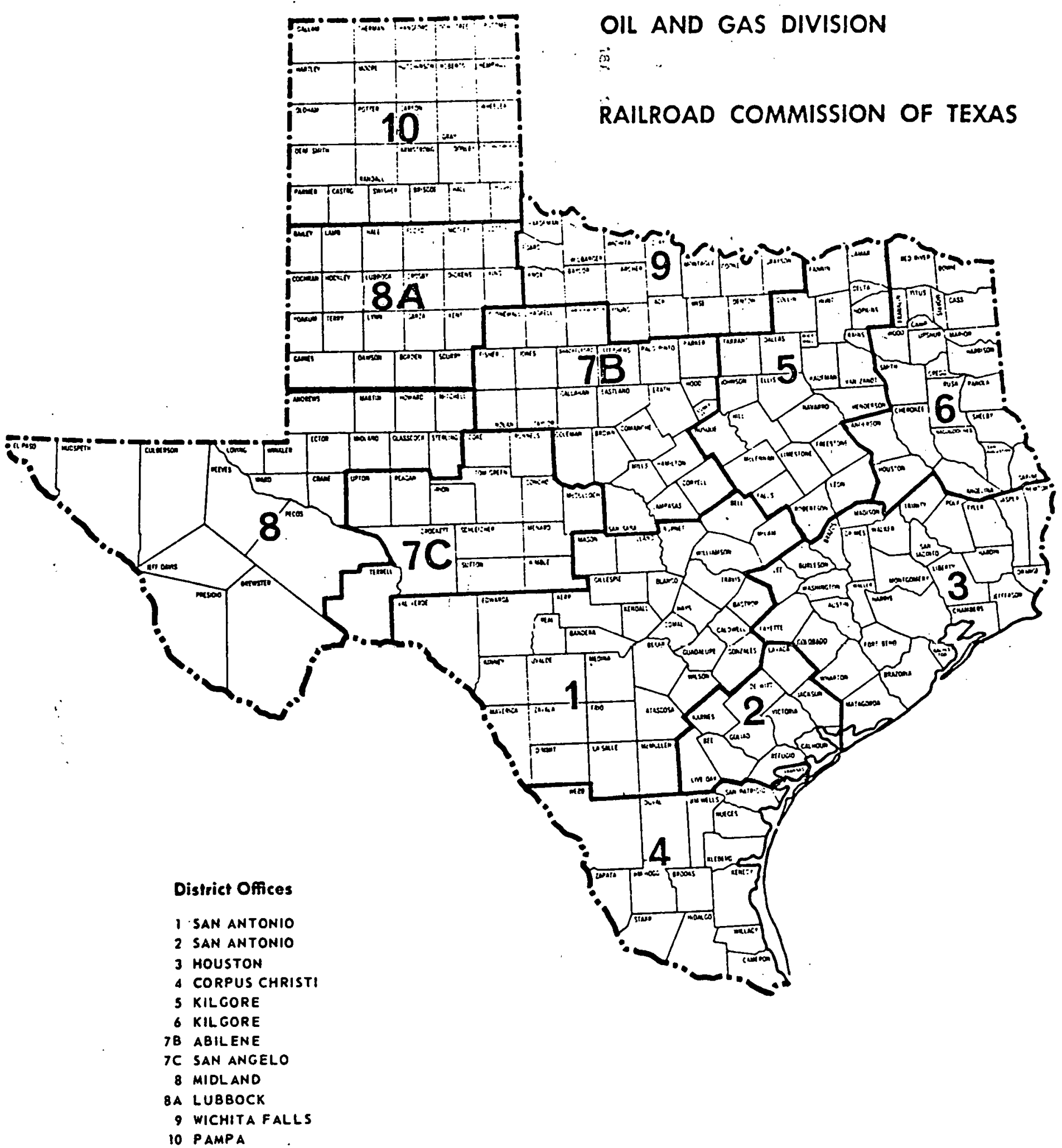


Limited distribution of this document is being made to key areas of interest such as State regulatory agencies and Petroleum Engineering and Geology schools in the region.

Copies of this document are available to the public through:

The National Technical Information Service

U.S. Department of Commerce

5285 Port Royal Road

Springfield, Virginia 22161

If you have any comments regarding usefulness of these data, please send them to:

Office of 0 il and Natural Gas

Office of Resource Applications

Department of Energy

12th and Pennsylvania Avenue, NW.

Washington, D.C: 20461

Your comments will be helpful in assessing feasibility of expanding

this sample study to other oil producing States. 
AEANDONED ONSRORE OIL FIELDS WITH OPER 250,000 BBL. CUAULATIVE PRODUCTION

DST DISC ABD. PLD NOM FIELD NAME

$01 \quad 19411959 \quad 53193001$ LENTZ

DST DISC ABD. FLD NJM FIELD NAME

01. 1913196816550001 CEDAR CREEK

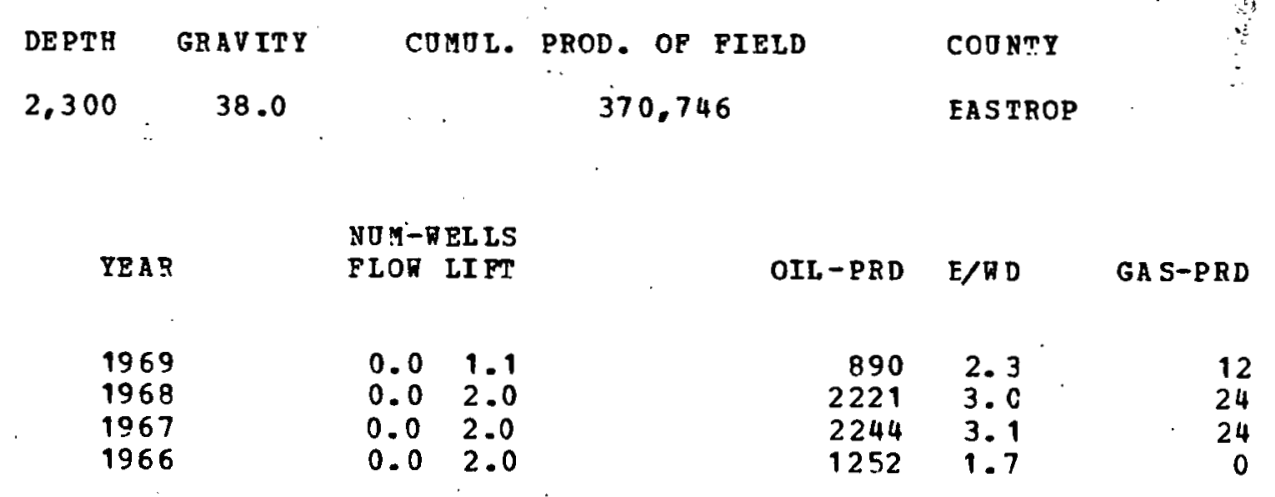

DEPTH GRAVITY COMUL. PROD. OF PIELD CONNTY

$1,650 \quad 34.0 \quad 341,977$

EASTROP

\begin{tabular}{|c|c|c|c|c|}
\hline YEAR & $\begin{array}{l}\text { NUM-WELLS } \\
\text { FLOW LIFT }\end{array}$ & OIL-PRD & E/TD & GAS-PRD \\
\hline $\begin{array}{l}1968 \\
1967 \\
1966\end{array}$ & $\begin{array}{ll}0.0 & 1.0 \\
0.0 & 1.0 \\
0.0 & 1.0\end{array}$ & $\begin{array}{l}110 \\
145 \\
390\end{array}$ & $\begin{array}{l}0.3 \\
0.4 \\
1.1\end{array}$ & . \\
\hline
\end{tabular}

2 FIELD (S)

712,723 CUMOLATIVE PROD. PROM ABANLONED FIELDS 
ABANDONED ONSHORZ OIL FIEIDS WTH OTER 250,000 BBL. CDMULATIVE PRODUCTION

DST DISC : 90. PLD NTM PIELD NAME

$01 \quad 1947 \quad 9962 \quad 47078001$ JOLIE?

COONTY: SALDHELI

DERTH GRAVITY

2,173

35.0

1 PIELD (S)
CUMOL. PROD. OP FIELD

353,817

$\operatorname{COONTY}$

CAL DNELL

\author{
353,817 COHDLATIVE PROD. PROM ABAYLONEL FIELDS
}


ABANDONED ONSHORE OIL FIELDS RITH OVER 250,000 BBL. COMOLATIVF FRODTCTION

DST DISC ABD. FLD UTI PIELD NAME

011959197589385200 THIRTEEN, EAST (3470 OLHOS)

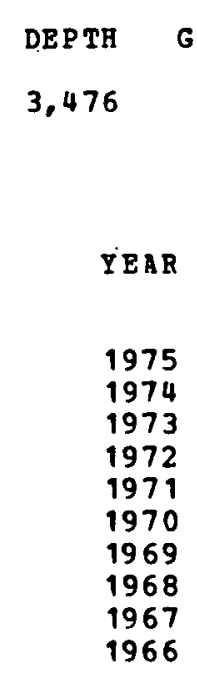

1 PIEID (S)

$\begin{array}{ccc}\text { GRAVITY COUOL. } & \text { PROD. OF FIELD } & \text { COONTI } \\ 43.0 & 1.513 .677 & \text { CIMMIT }\end{array}$

NOM-NELIS

PLON IIPT

OII-PRD E/TD

GAS-PRD

$\begin{array}{rr}0.5 & 1.2 \\ 1.6 & 3.4 \\ 2.3 & 3.1 \\ 3.5 & 2.4 \\ 2.6 & 10.0 \\ 7.8 & 3.1 \\ 10.8 & 0.2 \\ 10.2 & 0.1 \\ 10.0 & 0.0 \\ 8.6 & 0.7\end{array}$

775
13116

$\begin{array}{rr}13116 & 7.2\end{array}$

$\begin{array}{rr}51695 & 26.6 \\ 145639 & 67.4\end{array}$

28105861.2

$185822 \quad 46.6$

$\begin{array}{ll}185822 & 46.6 \\ 146450 & 36.5\end{array}$

$\begin{array}{ll}1: 46450 & 36.5 \\ 124064 & 33.5\end{array}$

12406433.2

$\begin{array}{rr}116918 & 32.0 \\ 89983 & 26.7\end{array}$

2815

30638

66332

$1 \$ 4596$

72294

334438

266730

212826

172996

87457 
ABANONRD ONSHORE OIL FIELJS MITH OVER 250,000 BBL. CUMOLATIVE PRODOCTION

DST DISC ABD. FLD NOM PIELD NAME

$01 \quad 19511970 \quad 41552001$ HTRDES

$\begin{array}{ccccc}\text { DEPTH } & \text { GRAVITY } & \text { COHOL. } & \text { PBOD. OF FIELD } & \text { COOATI } \\ 5.600 & 36.0 & & 543,984 & \text { FRIO }\end{array}$

YEAR

NUA-RELIS PLOW II FT

OIL - P RD

E/RE

GA S-PRD

1970

1968

1967

$\begin{array}{ll}0.0 & 0.1 \\ 0.0 & 1.7 \\ 0.0 & 4.0 \\ 0.0 & 4.0\end{array}$

$497 \quad 16.3$

$367 \quad 0.6$

$6825 \quad 4.7$

$8480 \quad 5.8$

135

2542

1865
1 PIEED (S)
543,984 CUHOLATIVE PROJ. PROA ABANDONED FIELDS 
ABANDONED ONSHORE OIL PIELDS MITH OVER 250,000 BBL. CUMOLATIVE PRODOCT ION

TST DISC.ABD FLO NIM PIELD NAME

$01 \quad 19291959 \quad 57082001$ MANPORD

CONNTY : GUADAT. TPE
DEPTH GRAVITY

2,615

37.0

1 PIEID (S)
COYOL. PROD. OF FIELD

434,825
COONTY

GOADAL UPE

434,825 CUAULATIVE PROD. PROA ABAMDORBD FIBLDS 
ARANDONED ONSHORE OIL PIEIDS QTTH OVER 250,000 BBL. COMULATIVE PRODUCTIDN

DST DISC ABD. FLD NTY SIPLD NAME

$01 \quad 1938 \quad 1975 \quad 63845001$ MTNSON

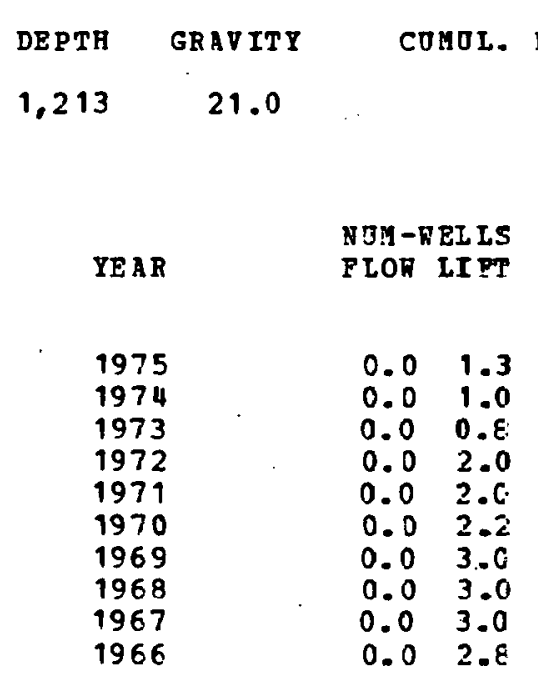

DEPTH

5,561

DST DTSC ABD. PLD NDM FIRLD NAME

GRAVITY

33.0

ก1 19601970 90300315 SAN MIGJEL CREEK (SEG D, C-15)
COMUL. PROD. OF PIELD

275.471
COONTY

RC MULLEN
299.574

$\begin{array}{rr}\text { OIL-PRD } & \text { E/RD } \\ & \\ 345 & 0.7 \\ 150 & 0.4 \\ 123 & 0.4 \\ 445 & 0.6 \\ 240 & 0.3 \\ 897 & 1.1 \\ 518 & 0.5 \\ 963 & 0.9 \\ 1985 & 1.8 \\ 1888 & 1.8\end{array}$

GA S-PRD

$\begin{array}{rr}0.7 & 8 \\ 0.4 & 6 \\ 0.4 & 5 \\ 0.6 & 5 \\ 0.3 & 6 \\ 1.1 & 12 \\ 0.5 & 12 \\ 0.9 & 10 \\ 1.8 & 12 \\ 1.8 & 0\end{array}$

YEAR
1970
1969
1968
1967
1966

NOM- VELLS

PLOH IIFT

$\begin{array}{ll}0.0 & 0.7 \\ 0.0 & 2.5 \\ 0.4 & 2.5 \\ 1.0 & 2.0 \\ 1.0 & 2.0\end{array}$

\section{OIT-PRD E/Й GAS-PRD}

$\begin{array}{rr}43 & 0.2 \\ 14063 & 15.4 \\ 27790 & 25.4 \\ 30292 & 27.7 \\ 24583 & 22.5\end{array}$


AGANDONED ONSBORE OIL FIELDS WITH OVER 250,000 BBL. CUMULATIVE PRODOCTION

DST DLSC ABD. FLD NIS FIELD NAME

$01 \quad 1958 \quad 1965 \quad 86217250$ STOCKDALE (AOSTIN CHALK)

$\begin{array}{cc}\text { DEPTH } & \text { GRAVITY } \\ 4,883 & 31.0\end{array}$

YEAR

1966
COONTY: WTLSON

DISTRICT 01
1 FIELD (S)

9 FIEIDS
COMOL. PROD. OF FIELD

285,985
NOM-TELIS

PLON IIFT

0.01 .9

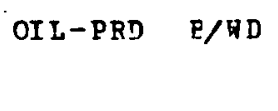

1228

1.8
GAS-P RD

0

$-0---0----$

285,985 CUMTLATIVE PRCD. PROM ABANCONEL FIEIDS

4,420,056 COMDLATIVE PROD. FROM ABANCONED PIELDS 
ABANDONED ONSHORE OIL PIELDS WITH OVER 250,000 BBL. COMOLATIVE PRODUCTICN

\begin{abstract}
DST DISC ABD. FLD NTM PIELD NAME
$0219421970 \quad 92214001$ TYNAN
\end{abstract}

DST DISC ABD. PLD NOM PIELD NAME

021952197215345500 CANHAN $(6800)$

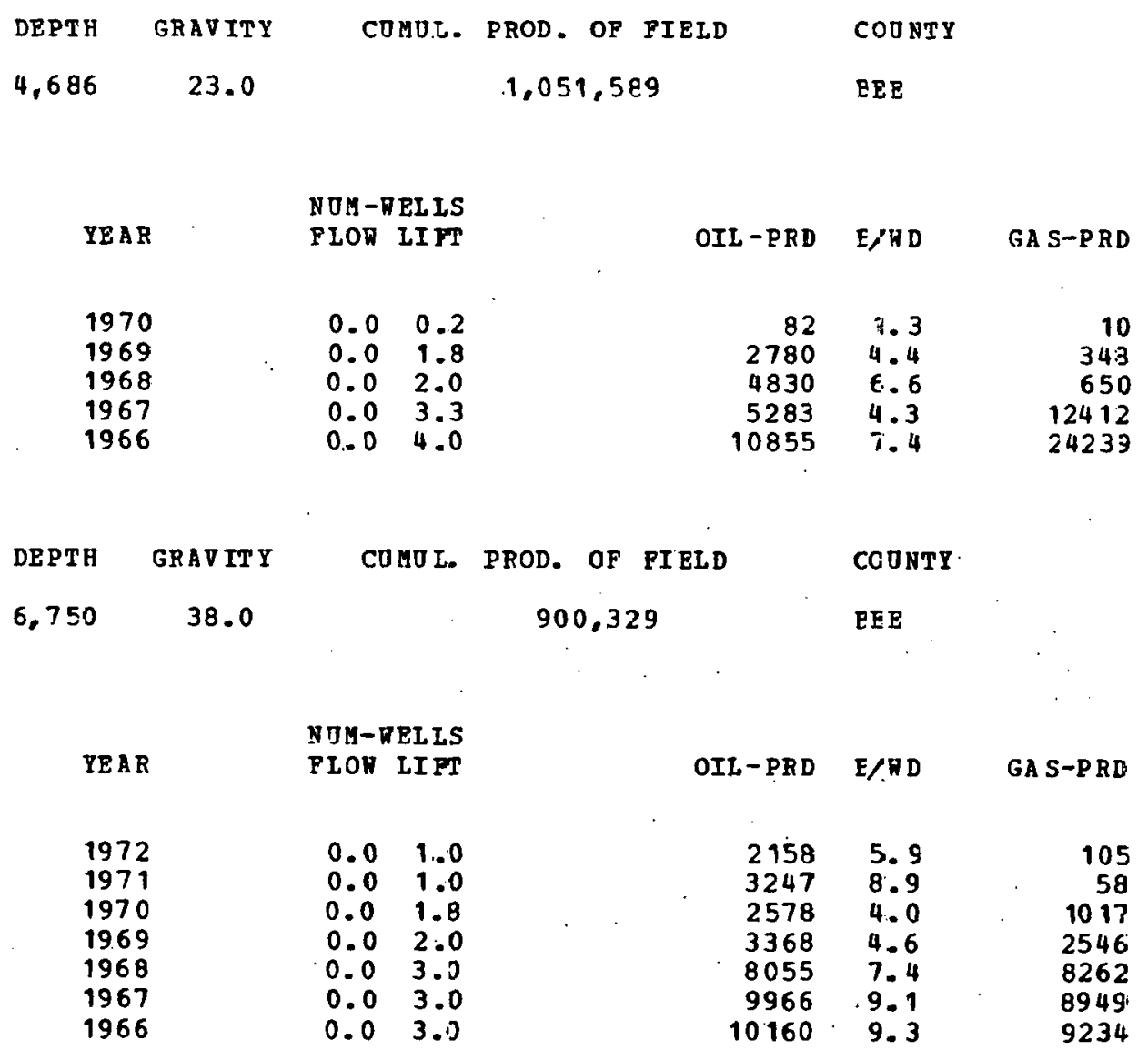


ABA NDONED ONSHORE OII FIELDS NITH OVE3 250,000 BBL. COMULATIVE PRODUCTION

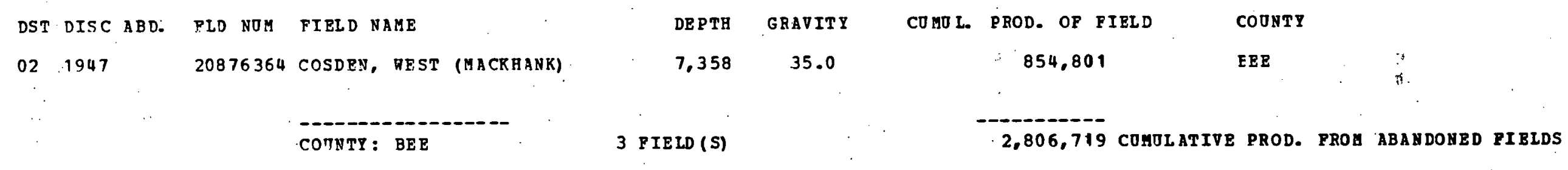


ABA NDONED ONSHORE OIL PIEIDS HITH OVER 250,000 BBL . COMOLATIVE PRODUCTION

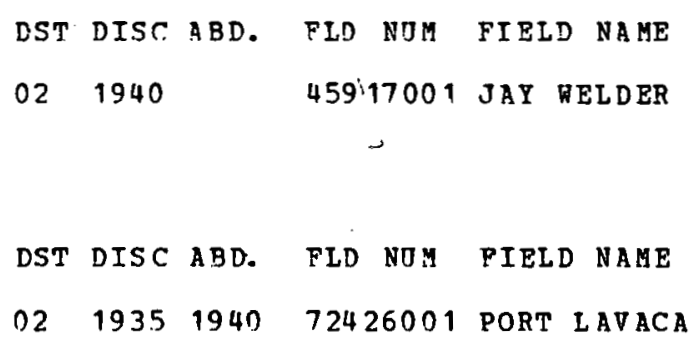

CODNT

CAL RCON

932,473

COUNTY

CAL HOUN

$\begin{array}{ccccc}\text { DEPTH } & \text { GRAVITY } & \text { COMOI. } & \text { PROD. } \text { OP PIELD } & \text { COUNTY } \\ 6,250 & 43.0 & & 363,071 & \text { CAL HOUN }\end{array}$


ABANDONED ONSHORE OII FIELDS WITH OVER 250,000 BBI. CUMOLATIVE PRODUCTION

DST DISC ABD. PLD NOM PIELD NALE

021951197640231000 HELEN GOHLRE (A-2)

DE P TH
8,163

Y E A R

1976
1975
1974
1973
1972
1971
1970
1969
1968
1967
1966

DEPTH

5,353

GRAVITY

43.0

31.0
COYOL. PROD. OF FIELD

$1,268,648$

CONNT

NOM-RELIS

FLON LIFT

0.110 .1

$0.5 \quad 1.5$

1. 12.6

$3.0 \quad 3.0$

$1.3 \quad 4.7$

0.56 .0

1.06 .0

$1.5 \quad 6.4$

$2.8 \quad 1.8$

$4.0 \quad 2.0$

4.02 .3

COMOL. PROD. OF PIELD

423,443

OIL-PRD E/WD

GAS-PRD

$\begin{array}{rrr}262 & 4.3 & 97 \\ 3546 & 4.9 & 16729 \\ 4062 & 3.0 & 6164 \\ 6731 & 3.1 & 76120\end{array}$

$\begin{array}{lll}6731 & 3.1 & 76120\end{array}$

$\begin{array}{lll}28796 & 13.1 & 239347\end{array}$

$\begin{array}{lll}35825 & 15.1 & 268075\end{array}$

$\begin{array}{lll}41486 & 16.2 & 255567\end{array}$

$\begin{array}{lll}25787 & 8.9 & 158788\end{array}$

$21327 \quad 13.0 \quad 202735$

$29605 \quad 13.5 \quad 200809$

$\begin{array}{lll}38334 & 16.6 & 1587.18\end{array}$

$021951 \quad 1976$ 40231429. HELEN GOHLKE (YEGOA K)

YEAR

1976

1975
NOM-TELIS

PLOR LIPT

$\begin{array}{ll}0.0 & 0.5 \\ 0.0 & 0.9\end{array}$
OIL-PRD E/WD

GAS-PRD

$1928 \quad 10.6$

$4481 \quad 13.4$ 
ABANDONED ORS HORE OIL PIELDS ITA OVER 250,000 BBL C CUOLATIVE PRODUCTION

DST DISC ABD: . FLD NOM FIBLD NAME.

O2. 19531972 . 40617875 HENZE $(4500)$

$\begin{array}{ccccc}\text { DEPTH } & \text { GRAVITY } & \text { COMOI. PBOD. OF FIELD } & \text { COUNTY } \\ 4.500 & 45.0 & & 255.723 & \text { CE NITT }\end{array}$

YEAR

NOH-RELIS

1972

1969

1968

1967

1966

3 FIEED (S)

$\begin{array}{rrr}\text { OII-PRD } & \text { E/RD } & \text { GAS-PBD } \\ 410 & 4.5 & \\ 646 & 4.2 & 0 \\ 14781 & 16.8 & 14053 \\ 9926 & 6.8 & 9698 \\ 14123 & 7.9 & 18170\end{array}$

COUNTY: DE NITT 
ABANDONED ONSHORE OIL PIELDS HTH OVER 250,000 BBL. COMULATIVE PRODUCTION

DST DTSC ABD. PID NOY PIELD NAME

$021948 \quad 1973 \quad 36065001$ GOTTSCHALT

DST DISC ABD. FID NOM PIELD NAME

$0219531970 \quad 11023497$ BOYCE (SLICK)

DEPTH
7,676
YEAR

1973
1972
1971
1970
1969
1968
1967
1965

GRAVITY

35.0

NUM-RELLS

FLON LIFT

$0.0 \quad 0.8$

$0.0 \quad 1.0$

0.01 .0

0.02 .2

0.03 .0

$\begin{array}{ll}0.0 & 3.0 \\ 0.0 & 3.0\end{array}$

1965

DEPTH GRAVITY

$7.557 \quad 33.0$

YEAR
1970
1963
1963
1967
1965

NOM-HELIS

FLOW IIFT

$0.0 \quad 0.5$

$\begin{array}{ll}0.1 & 1.1 \\ 1.0 & 2.0\end{array}$

$1.0 \quad 2.0$

2.02 .0
COONTY

GOL IA D
GOL IAD

OIL-PRD E/RD GAS-PRD

$\begin{array}{rrr}2377 & 8.7 & 12 \\ 3546 & 9.7 & 12 \\ 4509 & 12.4 & 12 \\ 4437 & 12.2 & 417 \\ 8539 & 10.8 & 17746 \\ 14933 & 13.6 & 28901 \\ 22329 & 20.4 & 45172 \\ 26165 & 23.9 & 43355\end{array}$

CUMUL. PROD. OF PIELD CODNTY

500,295 GOLIAD

OII-PRD E/RD GAS-PRD

$\begin{array}{rrr}166 & 0.9 & 2752 \\ 5657 & 13.3 & 6512 \\ 24463 & 22.3 & 11944 \\ 29741 & 27.2 & 13467 \\ 23861 & 16.3 & 17943\end{array}$


ABANDONED ONSHORE OIL FIELDS WITH OVER 250,000 BBL. COMOLATIVE PRODOCTION

DST DTSC AGD. TID NOM FIELD NAME

$021946 \quad 1968 \quad 72079001$ POEHLER

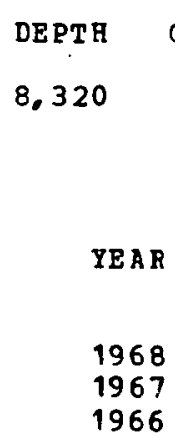

DEPTE

8,320

YEAR

1968
1967

1967
1966

GRAV ITY

26.0

CUMUI. PROD. OF FIEID

462,566

COONTY

DEPTH

GRAVITI

7,650

33.0

NUM-TRILS

FLOR IIFI

$$
\begin{array}{ll}
0.0 & 1.0 \\
0.0 & 0.0 \\
0.0 & 0.9
\end{array}
$$

EST DISC ABD. FLD NOM PIELD NAME

0219521970 . 28919125 EMMA HAYNES (LULING SAND)

YEAR
1970
1969
1968
1967
1966

4 PIELD (S)
COONTI: GOLIAD

$\begin{array}{cc}\text { CONOL. PROD. OF FIELD } & \text { COONTY } \\ 416,479 & \text { GOLIAD }\end{array}$

$\begin{array}{cc}\text { NOM-RELLS } \\ \text { FION IIFT } \\ \\ 0.0 & 1.0 \\ 0.0 & 1.0 \\ 0.0 & 1.0 \\ 0.0 & 1.0 \\ 0.0 & 1.0\end{array}$

$\begin{array}{rr}\text { OIL-PRD } & \text { E/RD } \\ & \\ 733 & 2.0 \\ 2876 & 7.9 \\ 2628 & 7.2 \\ 3337 & 9.1 \\ 3394 & 9.3\end{array}$

GA S-PRD

147

$5 \mathrm{B2}$

406

596
0 
ABANDORED ONSHORE OIL PIBIDS UITH OTER 250,000 BEL. COGOLATIVE PRODOCTIOH

DST DISC ABD. PLD NOH PIELD NAUE

02. 1969 1976. 96549400 REST RANCH, S. (FRIO 7050)

\begin{tabular}{|c|c|c|c|}
\hline DEPTR & GRAVITY & \multicolumn{2}{|c|}{ COHO L.: } \\
\hline .057 & 42.0 & & \\
\hline YEAR & & $\begin{array}{l}\text { NOM- } \\
\text { PLON }\end{array}$ & $\begin{array}{l}\text { EL IS } \\
\text { LI FT }\end{array}$ \\
\hline $\begin{array}{l}1976 \\
1975\end{array}$ & & $\begin{array}{l}0.0 \\
0.0 \\
0.0\end{array}$ & $\begin{array}{l}0.4 \\
1.0\end{array}$ \\
\hline $\begin{array}{l}1973 \\
1972 \\
1971 \\
1970 \\
1969 \\
1968\end{array}$ & & $\begin{array}{l}0.8 \\
2.8 \\
3.0 \\
3.0 \\
1.8 \\
0.2\end{array}$ & $\begin{array}{l}2.0 \\
0.3 \\
0.0 \\
0.0 \\
0.0 \\
0.1\end{array}$ \\
\hline
\end{tabular}

\begin{abstract}
DEPTH
GRAVITY
\end{abstract}

6,750

29. 0
CUMUL. PROD. OP FIELD

460,422
COONTY

$5.93,590$

JACRSON

O2: 1952197396612001 NESTHOPP RARCH

NOH-RELIS

PION LIPT

$0.4 \quad 0.0$

1.90 .0

$2.0 \quad 0.0$

$2.0 \quad 0.0$

$2.0 \quad 0.0$

$2.0 \quad 0.0$

$\begin{array}{ll}2.7 & 0.0 \\ 2.0 & 1.0\end{array}$
OIL-PRD E/RD

GAS-PRD

$\begin{array}{lll}1941 & 12.8 & 4833\end{array}$

$\begin{array}{lll}5933 & 16.3 & 18150\end{array}$

$\begin{array}{rrr}5933 & 16.3 & 18150 \\ 9850 & 21.6 & 8223\end{array}$

$83369 \quad 83.1 \quad 83786$

$121141110.6 \quad 63322$

$\begin{array}{lll}95611 & 87.3 & 51853\end{array}$

$1144668 \quad 132.1 \quad 85579$

$117101183.3 \quad 80075$

$13976153.2 \quad 5955$
OIL-PRD E/RD

GAS-PRD

$2874 \quad 18.9$

$36087 \quad 51.6$

3453347.3

3367046.9

$35886 \quad 49.2$

$26759 \quad 36.7$

$30327 \quad 31.2$

2733325.0
7850 209170 406497 379090 399691

262844

262844

219989 
ABA NDONED ONSGORE OIL PIBLDS ITH OVER 250,000 BBL. COUULATIVE PRODUCTICN

DST DISC ABD. PLD NOM FIELD NAMB

021959

58470400 MAURITZ, EAST (650ग)

DST DISC ABD. PLD NOH PIELD NAME

$0219591976 \quad 33603190$ GABRYSCR (5650)

DST DISC ABD. FID NDA PIELD NABE

021958196654458451 LOLI TA, DEEP (TEN CATE)

$\begin{array}{ccccc}\text { DEPTH } & \text { GRAVITY } & \text { COHOL. } & \text { PBOD. OF FIELD } & \text { COONTY } \\ 6,500 & 41.0 & & 404,279 & \text { JACKSON } \\ & & & & \\ \text { DEPTH } & \text { GRAVITY } & \text { COHOI. PROD. OF FIELD } & \text { COUNTY } \\ 5,656 & 28.0 & 305,684 & \text { JACKSON }\end{array}$

\begin{tabular}{lll} 
& \multicolumn{2}{c}{ NOL-RELIS } \\
YEAR & PLOR LIFT \\
& & \\
1976 & 0.4 & 0.0 \\
1975 & 1.0 & 0.0 \\
1974 & 1.0 & 0.0 \\
1973 & 1.0 & 0.0 \\
1972 & 0.8 & 0.3 \\
1971 & 0.6 & 0.4 \\
1970 & 0.0 & 1.0 \\
1969 & 0.0 & 1.0 \\
1968 & 0.2 & 0.5 \\
1967 & 2.0 & 0.0 \\
1966 & 2.0 & 0.0
\end{tabular}

OIL-PRD E/RD GAS-PED

$\begin{array}{lll}1662 & 10.9 & 7868\end{array}$

$\begin{array}{lll}3298 & 9.0 & 961\end{array}$

$\begin{array}{lll}16874 & 46.2 & 13151\end{array}$

$\begin{array}{lll}17157 \quad 47.0 & 17207\end{array}$

$20819 \quad 57.0 \quad 27080$

$29048 \quad 79.6 \quad 44971$

$\begin{array}{lll}13736 \quad 37.6 & 17316\end{array}$

$\begin{array}{lll}6540 & 17.9 & 2900\end{array}$

$\begin{array}{lll}3537 & 14.5 & 1304\end{array}$

$\begin{array}{rrr}3537 & 14.5 & 1304 \\ 10260 & 14.9 & 4735\end{array}$

$\begin{array}{rrr}10260 & 14.9 & 4735 \\ 24215 & 33.2 & 10055\end{array}$

DEPTH GRAVITI COEO PLOD. OP PIELD COONTI

$\begin{array}{llll}6,502 & 43.0 & 298,030 & \end{array}$

YEAR

NOH-RELLS

PIOR II FT

OIL-PRD. E/QD

GA S-PRD

1966
11045

6347 
ABANDONED ONSHORE OIL PIEIDS MITE OVER 250,000 BBL. COBULATIVE PRODOCTION.

DST DISC ABD. FLD NOM FIELD NAME

021959197251109306 LA WARD, NORTH (PIGPEN SAND)

DST DISC ABD. FLD NOH FIELD NAHE

02195619693312090 APPIING (ROPNICR I -D-)

\begin{tabular}{|c|c|c|c|}
\hline DEP TH & GRAVITY & \multicolumn{2}{|c|}{ CO LO L. } \\
\hline 5,816 & 32.0 & & \\
\hline & & NOM- & ELIS \\
\hline $\begin{array}{l}1972 \\
1971 \\
1970 \\
1969 \\
1968 \\
1967 \\
1966\end{array}$ & & $\begin{array}{l}0.0 \\
0.0 \\
0.0 \\
0.9 \\
0.0 \\
0.0 \\
1.7\end{array}$ & $\begin{array}{l}0.8 \\
2.0 \\
2.0 \\
1.6 \\
3.0 \\
3.6 \\
2.3\end{array}$ \\
\hline
\end{tabular}

DEPTH GRAVITY

8,562

37.0

COMUL. PROD. OP FIELD

232,097
COONTI

$29 \cdot 4,054$

JACKSON

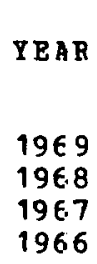

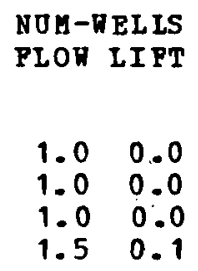

GAS-PRD

$\begin{array}{lrr}1627 & 4.5 & 34199\end{array}$

$\begin{array}{llr}4423 & 12.1 & 83786 \\ 7289 & 20.0 & 108459\end{array}$

$\begin{array}{lll}7289 & 20.0 & 108459 \\ 8478 & 14.7 & 100114\end{array}$ 
ABANDONFD ONSHORE OIL PIELDS RITH OVER 250,000 BBL. COMOLATIVP PRODUCTION

DST DISC ABD. FID NOH FIBLD NAME

02: 1965197533898902 S4 SADO, VEST (6000, LO.)

\begin{tabular}{|c|c|c|c|}
\hline DE PTH & GRAVITY & \multicolumn{2}{|c|}{ CO $90 \mathrm{~L}$} \\
\hline 5,969 & 32.0 & & \\
\hline YEA & & $\begin{array}{l}\text { NOG- } \\
\text { PLOR }\end{array}$ & $\begin{array}{l}\text { ELTS } \\
\text { IIFT }\end{array}$ \\
\hline $\begin{array}{l}1975 \\
1974 \\
1973 \\
1972 \\
197 \\
1970 \\
1960 \\
1968 \\
1967 \\
1966\end{array}$ & & $\begin{array}{l}0.0 \\
0.0 \\
0.0 \\
0.0 \\
0.0 \\
0.0 \\
2.0 \\
2.0 \\
2.4 \\
3.0\end{array}$ & $\begin{array}{l}0.2 \\
0.6 \\
0.0 \\
2.0 \\
2.0 \\
2.0 \\
0.0 \\
0.0 \\
0.0 \\
0.0\end{array}$ \\
\hline
\end{tabular}

\section{DEPTH GRAVITI}

7,274

45.0
D. OP. FIE

271,127

COOHTY

JaCKSOH
DST DISC ABD. FLD NOM PIELD NAME

021965197533989468 GANADO (F-17 SEG NiR)
9 FIELD (S)

\begin{tabular}{rrrrrr} 
YEAR & \multicolumn{1}{c}{ NOM-WELIS } & & \\
& FIOD LIPT & OII-PRD & E/AD & GAS-PBD \\
1975 & & & & & \\
1974 & 0.0 & 0.7 & 1324 & 5.4 & 1343 \\
1973 & 0.0 & 1.0 & 7995 & 21.9 & 6061 \\
1972 & 0.4 & 0.6 & 8065 & 22.1 & 8074 \\
1971 & 1.0 & 0.0 & 16784 & 46.0 & 20141 \\
1970 & 1.0 & 0.0 & 30421 & 83.3 & 26085 \\
1969 & 1.0 & 0.0 & 30316 & 83.1 & 28441 \\
1968 & 1.0 & 0.0 & 22056 & 60.4 & 12452 \\
1967 & 1.0 & 0.0 & 19821 & 54.3 & 9931 \\
1966 & 1.0 & 0.0 & 37274 & 102.1 & 36926 \\
& 1.0 & 0.0 & 53026 & 145.3 & 30009
\end{tabular}

$\begin{array}{rrr}\text { OIL-PRD } & \text { E/HD } & \text { GAS-PBD } \\ & & \\ 257 & 4.2 & 260 \\ 1481 & 7.0 & 612 \\ 4694 & 14.0 & 3214 \\ 9124 & 12.5 & 9574 \\ 11934 & 16.3 & 11864 \\ 15467 & 21.2 & 15375 \\ 20067 & 27.5 & 20966 \\ 18278 & 25.0 & 21278 \\ 35314 & 40.0 & 30263 \\ 103018 & \$ 4.1 & 78638\end{array}$

COHOL. PROD. OF FIELD

COONTY

254,964

JAC KSON 
ABANDONED ONSHORE OIL FIEIDS WITH OEER 250,000 BBL. CUMULATIVE PRODUCTION

DST DISC ABD. FLD NOM FIELD NAHE

$0219431967 \quad 72462001$ PORTER

DST DISC ABD. PLD NTM FIELD NAME

$0219371974 \quad 13652001$ BORNELI, SOOTH

\begin{tabular}{|c|c|c|c|c|}
\hline DEPTH & GRAVITY & CU MU L. & PROD. OF FIEID & COONTY \\
\hline 4.000 & 48.0 & & $2,211,162$ & KARNES \\
\hline
\end{tabular}

\begin{tabular}{|c|c|c|c|c|}
\hline YEAB & $\begin{array}{l}\text { NOA-DELIS } \\
\text { FLON LIPT }\end{array}$ & OIL-PRD & $E / H D$ & GAS-PRD \\
\hline $\begin{array}{l}1967 \\
1966\end{array}$ & $\begin{array}{ll}0.0 & 1.0 \\
0.0 & 1.0\end{array}$ & $\begin{array}{r}301 \\
1905\end{array}$ & $\begin{array}{l}0.8 \\
5.2\end{array}$ & $\begin{array}{l}7 \\
0\end{array}$ \\
\hline
\end{tabular}

$\begin{array}{ccccc}\text { DEPTH } & \text { GRAVITY } & \text { COGOL. } & \text { PROD. OP FIELD } & \text { COONTI } \\ 3,675 & 47.0 & 1,601,244 & \text { RARNES }\end{array}$

YER.F

NOM-VELLS

OII-PRD E/RD

GA S-PRD

1974

1973

1972

1971

1970

1969

1968

1967

1966

$\begin{array}{rr}48 & 1.6 \\ 881 & 1.4 \\ 1668 & 1.5 \\ 6195 & 5.7 \\ 7411 & 6.8 \\ 6519 & 6.0 \\ 22105 & 20.8 \\ 2815 & 7.7 \\ 1726 & 5.7\end{array}$

1
7
8
9
12
12
12
12
3606


ABANDONED ONSHORE CIL FIELDS TTH OVER 250,000 BBL. COHOLATIVE PRODUCTION

DST DISC ABD. FLD NOM PIELD NAME

$0219431970 \quad 72467001$ PORTER, SOUTH

DST DISC ARD. PID NOM FIELD NAME

021962197223473250 DAVY (EDTARDS)

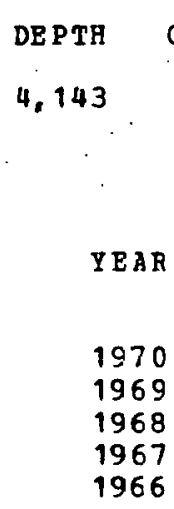

4,143

YEAR

1970

1969

1968

1967

1966

48.0

COMOL. PROD. OP EIELD

879,828

COONTY

NOM-REL ES
PION IIPT

DEPTH GRAVITY

$1.1,001$

38.0
CII-PRD E/RD

0.3

0.2

0.4

2.6

$4424 \quad 5.4$ $\begin{array}{ll}0.0 & 2.0 \\ 0.4 & 1.8\end{array}$

CUMOL PROD. OF FIELD

COUNTY

674,982

RAR NES
YE AR

1972

1971

1970

1969

1968

1967

1966
NOM-NELIS

FIOR LIFT

$0.0 \quad 0.9$

0.81 .5

1.90 .3

$3.0 \quad 0.8$

$3.0 \quad 2.9$

$3.7 \quad 2.3$

5.80 .3
OIL-PRD E/RD

GA S-PRD

$\begin{array}{rrr}7437 & 22.2 & 59227 \\ 8075 & 12.6 & 60283 \\ 11098 & 13.5 & 103702 \\ 15823 & 11.3 & 154253 \\ 41224 & 19.1 & 389935 \\ 84617 & 38.6 & 528502 \\ 105878 & 48.3 & 345996\end{array}$


ABANDONED ONSHORE OIL FIELDS ITH OVER 250,000 BBL, COMULATIVE PRODUCTICH

DST DISC ABD. FLD NOM PIELD NAHE

$02 \quad 1945$

44028001 HYSA

FID MTI PIELD NAEE

$021943 \quad 42415001$ GONDO CREEK

DST DISC ABD. PID NOH. PIELD NAHE

02. .1950

DE PTH

3,900 GRAVITY

26.0

DE P TH

6,582

GRAVITY

49.0

DEPTH

GRAVITI

6,494

37.0

7 FIELD (S)
COUOL. PROD. OP FIELD

459,170

COUOL. PROD. OP PIELD

354,694

COHOL. PROD. OP PIBLD

343,994

COONTY

RARRES 
ABANDONED ONSHORE OIL FEELDS WITH OVER 250,000 BBL COMULATIVE PRODUCTION

DST DISC ABD. FLD NOM FIELD NAME

$02 \quad 1945 \quad 1975 \quad 93777001$ VIENNA

DST DISC ABD. FLD NUM FIELD NAME

021947197573293560 PROVIDENT CITY (YEGOA)

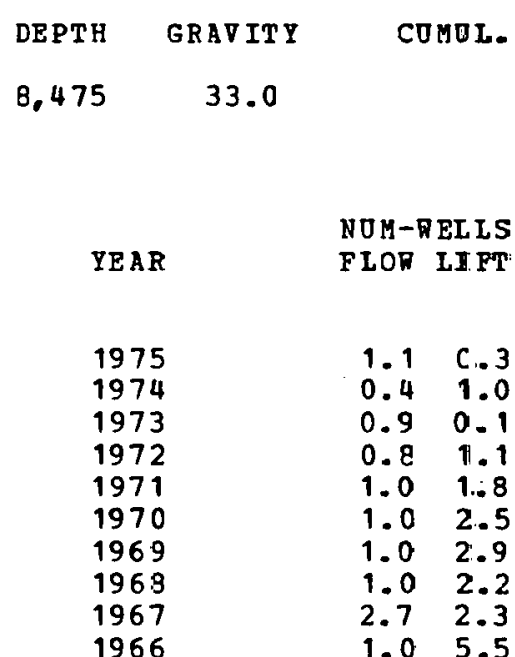

PROD. OF FIELD

$1,398,209$

COOHTY

IAVACA

$\begin{array}{rrr}\text { OIL-PBD } & \text { E/RD } & \text { GAS-P DD } \\ & & \\ 828 & 1.7 & 16256 \\ 1205 & 2.3 & 14887 \\ 1197 & 3.3 & 5938 \\ 3147 & 4.5 & 17364 \\ 5723 & 5.5 & 32313 \\ 6340 & 5.0 & 63819 \\ 10035 & 7.0 & 111016 \\ 12266 & 10.6 & 151455 \\ 12159 & 6.7 & 369453 \\ 18744 & 7.9 & 391624\end{array}$

COMOL. PROD. OF PIELD

826,950
CODNTI

IAVACA
5,495

45.0

\section{NUA-RELLS}

PLOW II?T

1975

1974

1973

1972

1971

1970

1969

1968

1967

1966
0.20 .0

1.00 .0

$1.0 \quad 0.1$

1.90 .2

$2.0 \quad 1.0$

$2.0 \quad 1: 2$

2. 81.2

3.30 .8

3. $5 \quad 0.0$

3.01 .0
OIL-PRD E/RD GAS-PRD

$\begin{array}{rrr}537 & 8.8 & 1000 \\ 5305 & 14.5 & 28828 \\ 5223 & 13.2 & 60725 \\ 9440 & 11.9 & 48717 \\ 21188 & 19.3 & 6022.3 \\ 25015 & 21.6 & 57359 \\ 30305 & 20.8 & 62180 \\ 29159 & 20.0 & 74955 \\ 30139 & 23.6 & 77522 \\ 23623 & 16.2 & 93796\end{array}$


ABANDONED ONSHORE OIL FIELDS MITH OVER 250,000 BBL. CUMOLATIPE PRODOCTICN

DST DISC ABD. FLD NTM FIELD NAHE

021950197278988001 ROSSER

\begin{tabular}{|c|c|c|c|}
\hline DE PTH & GRAVITY & \multicolumn{2}{|c|}{ CO nO I. } \\
\hline 5,284 & 42.0 & & \\
\hline & & NOM- & ELIS \\
\hline YEA 1 & & FLOD & \\
\hline $\begin{array}{l}1972 \\
1971 \\
1970 \\
1969 \\
1968 \\
1967 \\
1966\end{array}$ & $\begin{array}{l}2 \\
1 \\
0 \\
9 \\
8 \\
7 \\
6\end{array}$ & $\begin{array}{l}0.1 \\
1.0 \\
1.0 \\
1.0 \\
1.0 \\
1.0 \\
0.9\end{array}$ & $\begin{array}{l}0.0 \\
0.0 \\
0.0 \\
0.0 \\
0.0 \\
0.0 \\
0.0\end{array}$ \\
\hline
\end{tabular}

3 FIELD (S)
COONTY

352,075

LAVACA
OIL-PRD E/RD GAS-PRD

$\begin{array}{rrr}65 & 2.1 & 1 \\ 849 & 2.3 & 12 \\ 1783 & 4.9 & 12 \\ 710 & 1.9 & 8 \\ 1469 & 4.0 & 977 \\ 2115 & 5.8 & 5846 \\ 997 & 3.0 & 3088\end{array}$

2,577,234 COHOLATIVE PROD. FROA ABANDONED FIELDS 
ABA NDONED ONSHORE OIL FIELDS RTH OVER 250,000 BBI. COHOLATIVE PRODUCTION

DST DISC ABD. PID NOM PIELD NAUE

021953197063354324 MT. LUCAS (BCIVER SAYD)

DST DISC ABD. PID NOH PIBLD NAME

$02 \quad 1953$

63354378 MT. LOCAS (VICKSBORG)

DST DISC ABD. FID NOH PIELD NAME

02 $19541970 \quad 18765370$ CLAYTON [QDEEN [IT

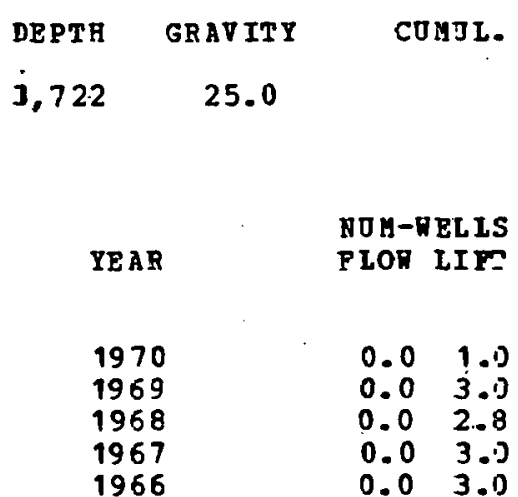

DEPTH

GRAVIT Y

3.748

25.0

DEPTH

GRAVITY

5,119

44.0

COMOI. PROD. OP FIELD

647.248

$$
1,365,862
$$

COUNTY

IIVE OAK

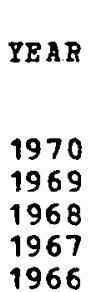

NTM-RELIS

FLOR LIFT

E/MD

1970

1969

1968

1966

$0.0 \quad 1.7$

$2.8 \quad 8.6$

$3.0 \quad 9.0$

3. $0 \quad 9.0$

$3.09: 0$

$\begin{array}{rrr}1779 & 2.9 & 1086 \\ 8839 & 2.1 & 7003 \\ 11273 & 2.6 & 3366 \\ 12388 & 2.0 & 10538 \\ 14046 & 3.2 & 10843\end{array}$


AGANDONED ONSHORE OIL PIELDS HITH OVER 250,000 BBL. COMULATIVE PRODOCTION

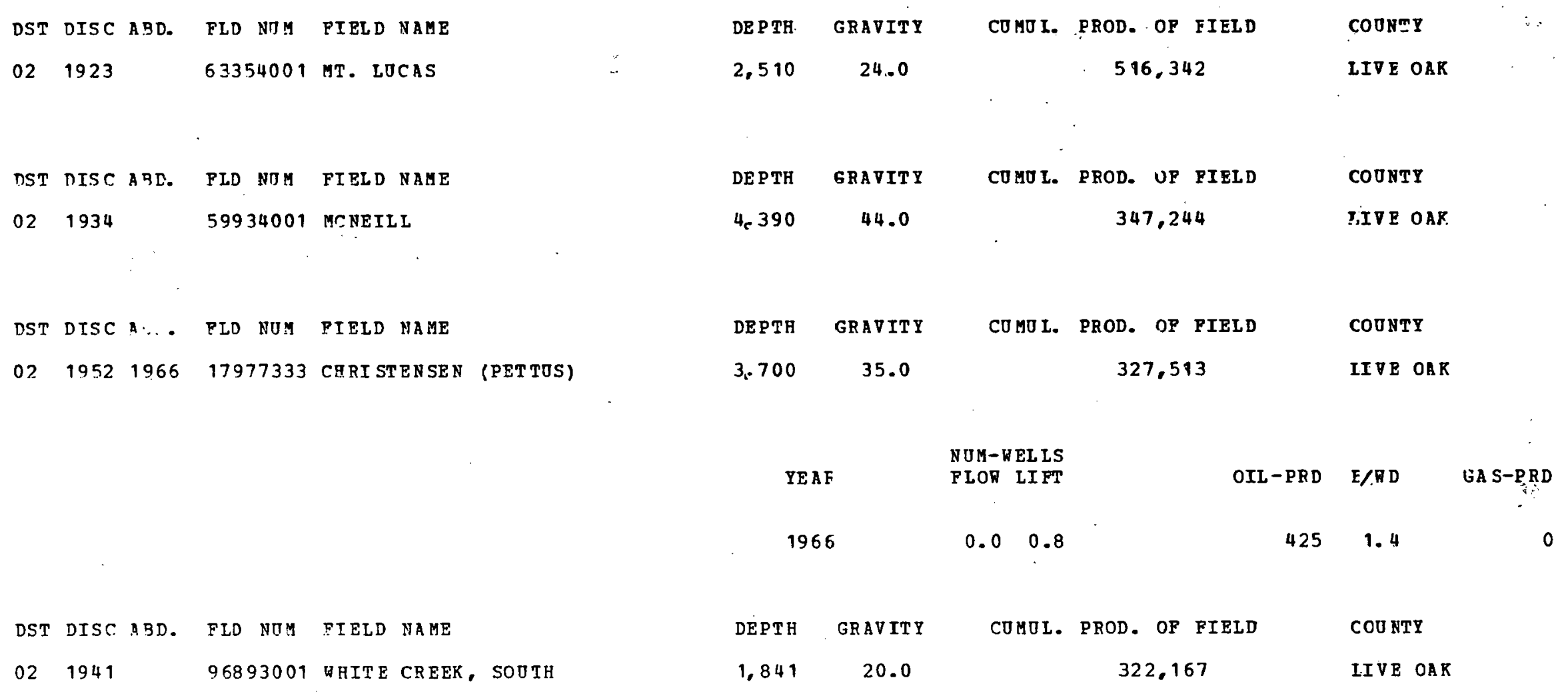


ABANDONED ONSHORE OIL FIELDS VITH OVER 250,000 BBL. COMULATIVE PRODUCTION

DST DISC ABD. FLD NOM PIELD NAME

$02 \quad 1939 \quad 1975 \quad 50975001$ LA ROSA

DST DISC ABD. FLD NUM FIRLD NAME

$021954 \quad 1972 \quad 51512836$ LARE PASTURE (FT-590)

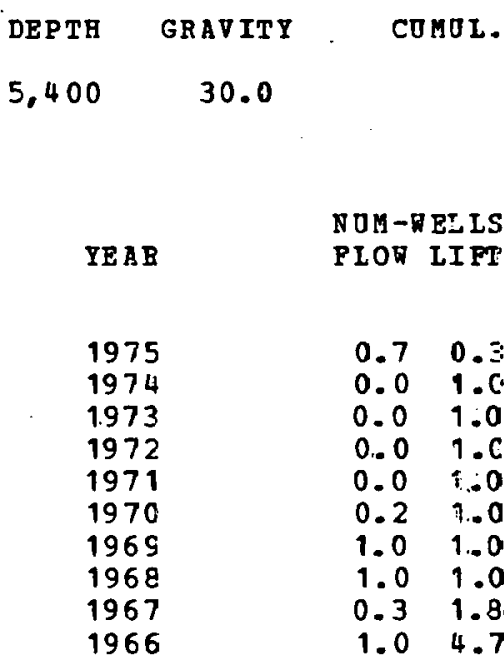

PROD. OP FIELD

COONTY

$17,350,446$

BEPOGIO
COMUI. PROD. OF PIELD

$1,187,417$

$\begin{array}{rrr}\text { OIL - PRD } & \text { E/RD } & \text { GAS-PRD } \\ & & \\ 9418 & 25.8 & 14505 \\ 728 & 2.0 & 146 \\ 578 & 1.6 & 120 \\ 576 & 1.6 & 121 \\ 1853 & 5.1 & 556 \\ 2534 & 6.0 & 16565 \\ 5978 & 8.2 & 36441 \\ 7302 & 10.0 & 55213 \\ 9393 & 11.9 & 90547 \\ 9392 & 4.5 & 65937\end{array}$

COONTY

REP UGIO

\begin{tabular}{lll} 
& \multicolumn{2}{c}{ NDM-TELLS } \\
YEAR & FLOH LIFT \\
& & \\
1972 & 0.0 & 1.5 \\
1971 & 0.0 & 2.0 \\
1970 & 0.0 & 2.1 \\
1969 & 0.0 & 3.0 \\
1968 & 0.0 & 3.0 \\
1967 & 0.0 & 3.0 \\
1966 & 0.0 & 3.2
\end{tabular}

$\begin{array}{rrr}\text { OIL-PAD } & \text { E/HD } & \text { GAS-PRD } \\ & & \\ 12065 & 22.0 & 60690 \\ 22476 & 30.8 & 111492 \\ 25307 & 33.3 & 52197 \\ 42519 & 58.8 & 57903 \\ 32191 & 29.4 & 30436 \\ 35635 & 32.5 & 46351 \\ 33643 & 29.1 & 47467\end{array}$


ABANDONED ONSHORE OIL FIEIDS NITH OVER 250,000 BBL. COMOLATIVE PRODUCTION

DST DISC ASD. FID NOM PIELD HAHE

021961197150975111 LA ROSA (DRISCOLI)

DST DISC ABD. PLD NOM FIBLD FAME

021960196850975037 IA ROSA (BIRDIE SAND)

DEPTH GRAVI

8. 232

45.0

YE R.R

\section{1}

1970

1969

1958

1957
1966

6,831

GRAVIT

44.0
COHUL. PROD. OP FIEID

598,226

CCUNTI

FEP OGIO

\begin{tabular}{|c|c|c|c|c|}
\hline & NOH-RELIS & & & \\
\hline YEAR & PLON LIPT & OIL-PRD & $E / D D$ & GAS-PRD \\
\hline $\begin{array}{l}1968 \\
1967 \\
1966\end{array}$ & $\begin{array}{ll}0.8 & 0.3 \\
1.0 & 0 . .3 \\
2.9 & 0.8\end{array}$ & $\begin{array}{r}5973 \\
52595 \\
46878\end{array}$ & $\begin{array}{r}14.0 \\
108.1 \\
34.2\end{array}$ & $\begin{array}{l}11289 \\
59032 \\
43833\end{array}$ \\
\hline
\end{tabular}


ABANDOYED ONSAORE OIL ZIELDS MITB OVER 250,000 BBL. COUOLATIVE PRODUCTION

DST DISC ABD. FID NOH PIELD NAME

021961197651512684 LAKE PASTURE (PT-5?0)

DST DISC ABD. PID NOS PIELD MA HE

$021951 \quad 197129938055$ PAGAR (GRETA SAND)

\begin{tabular}{|c|c|c|c|c|}
\hline DEPTE & GRAVITY & cosol. & PROD. OF PIELD & COONTY \\
\hline 8 & 38.0 & & 403,405 & REPOGIC \\
\hline
\end{tabular}

YEAR

NOA-RELLS
PLOO LIFT

OIL-PRD E/RD

GA S-PRD

$0.5 \quad 0.0$

0.90 .0

1.10 .0

$2.0 \quad 0.0$

2.20 .0

$2.0 \quad 0.3$

2.01 .0

1.39 .9

1.91 .1

$\begin{array}{ll}2.3 & 0.9 \\ 3.0 & 1.0\end{array}$

1968

1967

1966

DEPTH GRAVIT Y

CO HO L. PROD. OF TIELD

COUHTY

4,709

24.0

379,665

BEP OGIO

\begin{tabular}{lll} 
& \multicolumn{2}{c}{ NUM-NEZLS } \\
YEAR & PLOR & II TT \\
& & \\
1971 & 2.0 & 1.0 \\
1970 & 2.0 & 2.0 \\
1969 & 2.0 & 2.0 \\
1968 & 0.8 & 3.2 \\
1967 & 0.0 & 4.0 \\
1966 & 0.0 & 4.0
\end{tabular}

$\begin{array}{rrr}\text { OIL-PRD } & \text { E/RD } & \text { GR S-PRD } \\ & & \\ 375 & 0.3 & 274 \\ 3033 & 2.1 & 2063 \\ 5448 & 3.7 & 4417 \\ 9344 & 6.4 & 6576 \\ 11381 & 7.8 & 8050 \\ 17140 & 11.7 & 10291\end{array}$


ABANDONED ONSBORE OIL FIELDS IITH OVER 250,000 BBL. COHOLATIPB PRODOCTION

DST DISC ARD. PLD NOM PIELD NAME

$0219661975 \quad 50975777$ LA ROSA (6400)

\begin{tabular}{|c|c|c|c|}
\hline DE PTH & GRAV ITY & \multicolumn{2}{|c|}{ COHOL. } \\
\hline 6,405 & 45.4 & & \\
\hline TEAR & & $\begin{array}{l}\text { NOA- } \\
\text { PLOD }\end{array}$ & $\begin{array}{l}\text { EL LS } \\
\text { II FI }\end{array}$ \\
\hline $\begin{array}{l}1975 \\
1974 \\
1973 \\
1972 \\
1971 \\
1970 \\
1969 \\
1968 \\
1967 \\
1966\end{array}$ & & $\begin{array}{l}0.0 \\
0.0 \\
0.1 \\
0.0 \\
0.5 \\
2.2 \\
2.4 \\
1.8 \\
0.1 \\
0.0\end{array}$ & $\begin{array}{l}0.05 \\
0.8 \\
0.3 \\
1.1 \\
0.5 \\
0.5 \\
0.00 \\
0.0 \\
0.3 \\
0.1\end{array}$ \\
\hline
\end{tabular}

COU RTY

BEF OGIO
DST DISC ABD. PLD NTE FIELD NAHE

$02 \quad 19591974 \quad 75654528$ REPTGIO NEA (6100 STRIHGER 1)
DE PTH

6,036
GRAVITI

39.0
305,206

$\begin{array}{rrr}\text { OIL-PBD } & \text { E/RD } & \text { GAS-PRD } \\ & & \\ 1619 & 8.9 & 15688 \\ 3492 & 11.5 & 42460 \\ 631 & 5.2 & 3032 \\ 9188 & 23.2 & 26965 \\ 19901 & 54.5 & 31398 \\ 49326 & 50.7 & 63000 \\ 59470 & 67.4 & 54350 \\ 106558 & 159.2 & 166501 \\ 51103 & 420.0 & 53140 \\ 3776 & 124.1 & 5579\end{array}$

\begin{tabular}{|c|c|c|}
\hline $\mathbf{C X R}$ & $\begin{array}{l}\text { NOB- } \\
\text { PLON }\end{array}$ & $\begin{array}{l}\text { IEL IS } \\
\text { LI PI }\end{array}$ \\
\hline $\begin{array}{l}1974 \\
1973 \\
1972 \\
1971 \\
1970 \\
1969 \\
1968 \\
1967 \\
1966\end{array}$ & $\begin{array}{l}0.0 \\
0.0 \\
0.0 \\
0.0 \\
0.0 \\
0.0 \\
0.7 \\
1.1 \\
1.0\end{array}$ & $\begin{array}{l}1.3 \\
2.0 \\
2.0 \\
3.0 \\
2.7 \\
1.5 \\
2.0\end{array}$ \\
\hline
\end{tabular}

CUAOL. PROD. OP PIBLD

276,778
COO BTY

REPUGIO

YEAR


1974
1973
1972
1971
1970
1969
1968
1967
1966

NTB-REL IS

0.01 .3

0.02 .0

$0.0 \quad 3.0$

0.02 .7

$0.7 \quad 2.7$

1.02 .0

$\begin{array}{rrr}\text { OIL-PRD } & \text { E/RD } & \text { GAS-PRD } \\ & & \\ 3383 & 7.0 & 11166 \\ 5011 & 6.9 & 13397 \\ 9384 & 12.9 & 8649 \\ 14496 & 13.2 & 15403 \\ 9989 & 10.3 & 17379 \\ 6022 & 8.6 & 35413 \\ 30444 & 25.0 & 32292 \\ 23531 & 20.4 & 26223 \\ 17019 & 15.5 & 26152\end{array}$


ABANDONED ONSHORE OIL FIEIDS RITH OVER 250,000 BBL. CJMOLATIVE PRODOCTION

\begin{abstract}
DST DISC ABD. PLD NITM PIBLD NAHE
$021941 \quad 1973 \quad 75598001$ REPTGIO, NORTH
\end{abstract}

DST DISC ABD. PLO NOM PIBLD NABE

$02 \quad 1940$

DEP.TH
5,664

IEAR

1973
1972
1971
1970
1969
1968
1967
1966

$\begin{array}{cr}\text { DEPTH } & \text { GRAVIT Y } \\ 5.895 & 29.0\end{array}$

10 FIELD (S)

\begin{tabular}{|c|c|c|c|}
\hline GRAVITY & CO HO L. & PROD. OP FIELD & COONTY \\
\hline 41.0 & & 275,485 & REF OGIO \\
\hline
\end{tabular}

$\begin{array}{ll}\text { NOH- RELLS } \\ \text { PLOR LIPT } \\ \\ \\ 0.0 & \\ 0.0 & 0.3 \\ 0.0 & 1.0 \\ 0.0 & 1.0 \\ 0.0 & 1.0 \\ 0.0 & 1.0 \\ 0.0 & 1.0 \\ 0.0 & 1.0\end{array}$

OIL-PRD

E/RD

GAS-PRD

$\begin{array}{rr}75 & 0 \\ 782 & 2 \\ 972 & 2 \\ 2060 & 5 \\ 2521 & 6 \\ 1792 & 4 \\ 1779 & 4 \\ 2357 & 6 .\end{array}$

1125

9754

12341

10540

12489

9591

14936

14752.
COUOL. PEOD. OF FIELD

266,007
COONTI

REPOGIO
$21,468,640$ COMOLATIVE PROD. FROA ABANCONED PIELDS 
ABANDONRD ONSHORE OIL PIELDS NITH OVIR 250,000 BRL. COMULATIVE PRODOCTION

DST DISC ABD. PLD NDM PIELD NAME

$02 \quad 1930 \quad 197159371001$ MCPADDIN

DST DISC ABD. PLD NOH FIELD NAME

021933196919557001 COLETTO CREEK

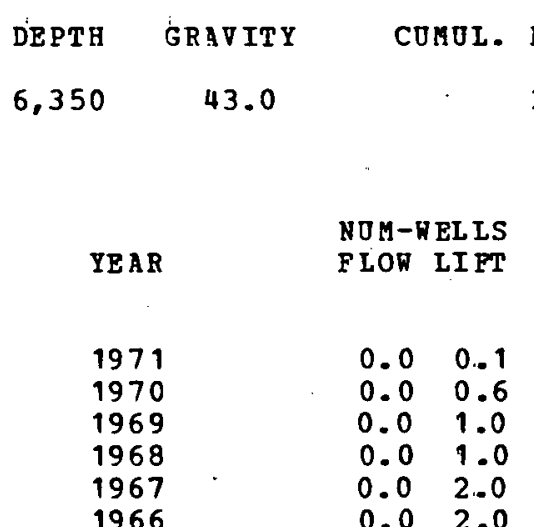

PROD. OF PIELD

$26,216,578$

$\operatorname{CCONTI}$

VICTORIA

$\begin{array}{rrr}\text { OIL-PRD } & \text { E/WD } & \text { GAS-PRD } \\ & & \\ 62 & 2.0 & 1 \\ 816 & 3.8 & 19800 \\ 1749 & 4.8 & 29295 \\ 2217 & 6.1 & 28182 \\ 4898 & 6.7 & 17719 \\ 4796 & 6.6 & 29792\end{array}$

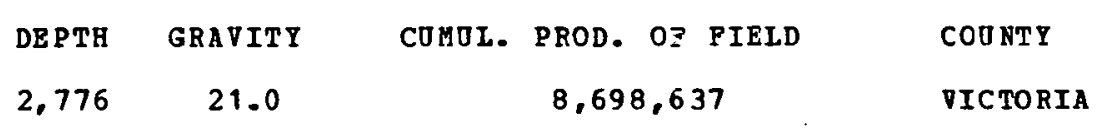

YEAR

NOM-RELIS

OIL-PRD E/PD

GAS-PRD

1969

1968

.1967

$\begin{array}{rr}5.4 & 7.3 \\ 15.9 & 30.7\end{array}$

$13.0 \quad 32.2$

11.133 .9

70835

279315

$\begin{array}{lll}265950 & 16.1 & 122708 \\ 262865 & 16.0 & 105545\end{array}$

15.2
16.4
16.1 
ABANDONED ONSHOEE OIL PIELES VITH OVER 250,000 BBL - CCMOLATIVE PRODUCTION

DST DISC ABD. FLD NDM FIELD NAME

$0219491974 \quad 7064001$ BEN SHELTON

DST DISC ABD. PLD NOH PIELD NAME

$0219391975 \quad 19744001$ COLOGN

\begin{tabular}{|c|c|c|c|c|}
\hline DE PTH & GRAVITY & COMO L. & PROD. OF FIELD & COONTY \\
\hline & 44. & & $1,234,983$ & A \\
\hline
\end{tabular}

\begin{tabular}{|c|c|c|}
\hline & NOM -1 & ELIS \\
\hline Y E A R & FLOด & LIFT \\
\hline $\begin{array}{l}1974 \\
1973 \\
1972 \\
1971 \\
1970 \\
1969 \\
1968 \\
1967 \\
1966\end{array}$ & $\begin{array}{l}0.0 \\
1.3 \\
2.0 \\
2.0 \\
2.0 \\
2.0 \\
2.0 \\
2.0 \\
2.0\end{array}$ & $\begin{array}{l}0.2 \\
0.7 \\
0.26 \\
1.0 \\
1.0 \\
1.0 \\
1.0 \\
1.0 \\
1.0\end{array}$ \\
\hline
\end{tabular}

DEPTH GRAVITY

CUMOL. PROD. OF FIELD

$1,179,957$
COUNTY

VICTORIA
GAS-PRD

$\begin{array}{rrr}222 & 3.6 & 375 \\ 6748 & 9.2 & 11438 \\ 7704 & 8.2 & 13023 \\ 10969 & 10.0 & 18539 \\ 12349 & 11.3 & 20853 \\ 12049 & 11.0 & 20362 \\ 12342 & 11.3 & 20857 \\ 8886 & 8.1 & 15015 \\ 11004 & 10.0 & 17058\end{array}$

$4.827 \quad 25.0$

\begin{tabular}{|c|c|c|}
\hline I BAR & $\begin{array}{l}\text { NOM- } \\
\text { FLON }\end{array}$ & $\begin{array}{l}\text { ELIS } \\
\text { LI FT }\end{array}$ \\
\hline $\begin{array}{l}1975 \\
1974 \\
1973 \\
1967 \\
1966\end{array}$ & $\begin{array}{l}0.0 \\
0.0 \\
0.0 \\
0.0 \\
0.0\end{array}$ & $\begin{array}{l}0.1 \\
0.1 \\
0.1 \\
1.3 \\
2.0\end{array}$ \\
\hline
\end{tabular}

OIL-PRD E/HD GAS-PRD

$\begin{array}{rr}33 & 1.1 \\ 61 & 2.0 \\ 291 & 9.6 \\ 736 & 1.5 \\ 2726 & 3.7\end{array}$


ABANDONED ONSHORE OIL FIEIDS MITH OVER 250,000 BBL. COUDLATIVE PRODUCT ION

DST DISC ABD. FLD NOM FIRID NAME

021961197159373580 MCPADDIN, NORTH (5550)

\begin{tabular}{|c|c|c|c|}
\hline DEPTH & GRAV IT I & \multicolumn{2}{|c|}{ COHOL. } \\
\hline 5,571 & 39.0 & & \\
\hline & & חסו- & ELIS \\
\hline YEAR & & FIOR & LI FT \\
\hline $\begin{array}{l}1971 \\
1970 \\
1969 \\
1968 \\
1967 \\
1966\end{array}$ & & $\begin{array}{l}0.0 \\
0.0 \\
0.3 \\
2.4 \\
3.7 \\
3.1\end{array}$ & $\begin{array}{l}0.5 \\
2.7 \\
4.2 \\
2.4 \\
2.2 \\
2.8\end{array}$ \\
\hline
\end{tabular}

DE PTH

8,180

\section{GRAVITI}

34.0
COHOL. PROD. OP PIELD

345,851
COU NTI

VICTORI A

021953196740239456 HELEN GOHLKE, ค. (NILCOX A-2)

YEAR

1967
NOH-RELIS

0.10 .5
383,596

OIL - PRD

E/R D

$$
\begin{array}{r}
7 \\
22 \\
25 \\
35 \\
44
\end{array}
$$

112
7159 22131 25318 35016

0.6
7.1

7.1
13.7

14.4

16.4

20.7

COO FTP

VICTORIA

\section{4} 19400 32819 65164 109985 86124 
ABANDONED ONSHORE OIL PIELDS NITH OVER 250,000 BBL. COMOLATIVE PRODUCTION

DST DTSC ABD. FLD NTM FIELD NAME

n2 1949197593725825 VICTORIA (4800 SAND)

$\begin{array}{cccc}\text { DEPTH } & \text { GRAVITI } & \text { COHUL. PROD. OF FIELD } & \text { COU FTY } \\ 4,800 & 24.0 & 278,728 & \text { VICTORIA }\end{array}$

N JE- RELIS

FLOT LIFT

OIL -PRD E/RD

GA S-PBD

1975

1974

1973

1972

1971

1970

1969

1968

1967

1966

CODNTI: VICTORIA

7 TIELD (S)

DISTRICT 02

55 EIEIDS

$\begin{array}{llrlr}0.0 & 0.3 & 91 & 1.0 & 18 \\ 0.0 & 0.8 & 1279 & 4.7 & 256 \\ 0.0 & 0.8 & 1598 & 5.8 & 320 \\ 0.0 & 1.9 & 2670 & 3.8 & 462 \\ 0.0 & 2.0 & 3047 & 4.2 & 427 \\ 0.0 & 1.8 & 4211 & 6.6 & 417 \\ 0.0 & 1.9 & 4435 & 6.3 & 425 \\ 0.0 & 1.4 & 3905 & 7.6 & 635 \\ 0.0 & 1.9 & 4201 & 6.0 & 843 \\ 0.0 & 2.0 & 3577 & 4.9 & 926\end{array}$

$38,338,730$ CUMOLATIVE PROD. FROA ABANDONED FIELDS

84,942,202 COMOLATIVB PROD. FROM ABANDONED FIELDS 
ABANDONED ONSRORE OIL PIELDS WITA OVIR 250,000 BBL. CUMULATIVE PRODUCTION

DST DISC ABD. FLD NJM FIELD NAME

031945197265248001 NE OL

$\begin{array}{ccccc}\text { DEPTH } & \text { GṚAVITY } & \text { COMUL. } & \text { PROD. OF FIELD } & \text { COUNTY } \\ 9,150 & 34.6 & 3,379.620 & \text { AOSTIN }\end{array}$

YEAR

NOM-TELIS

OIL-PRD E/RD

GAS-PRD

1972

1971

1970

1969

1968

1967

1966

COONTY : AOSTIN

1 PIELD (S)

$\begin{array}{rrrrr}0.0 & 1.2 & 137 & 0.3 & 27 \\ 0.0 & 1.5 & 1720 & 3.1 & 345 \\ 0.0 & 4.4 & 4454 & 2.8 & 3718 \\ 0.0 & 7.0 & 11566 & 4.5 & 18359 \\ 0.6 & 7.8 & 12511 & 4.1 & 16250 \\ 0.5 & 8.0 & 13244 & 4.3 & 36064 \\ 0.0 & 9.0 & 17408 & 5.3 & 30879\end{array}$

3,379,620 CUMULATIVE PROD. FROM ABANCONEL PIELDS 
ABANDOHED ONSHORE OIL PLĖLDS RTTH OVER 250,000 BBL. COMOLATIVE PRODOCTICA

DST DISC ABD. PLD NOH PIELD NAGE

031941197417915442 CHOCOLATE BAYOU( 月о.PHS. VI-2-Z 9,920
GRAVITY

38.0
COHDL. PROD. OF PIELD

$$
5,250,755
$$

CCONTY

ERAZORIA :

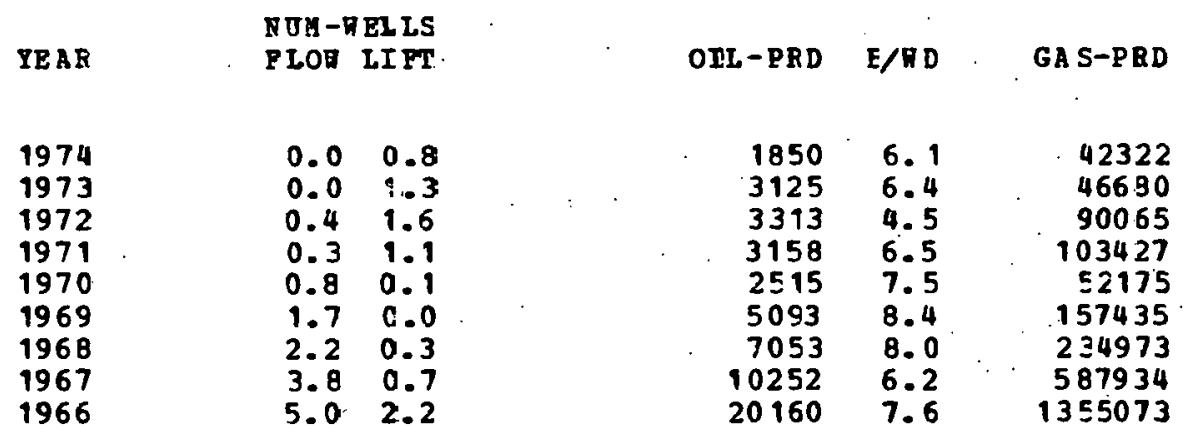

DST DISC ABD. PLD YOI PIELD NARE

031953197686530001 STRATTON RIDGE, SODTE

$\begin{array}{cc}\text { DEPTH } & \text { GRAVITY } \\ 5.619 & 22.8\end{array}$

YEAR

\section{6}

1976

1974

1973

1972

1971

1970

1969

1968

1967

1966
22.8

CDAOI. PROD. OP FIELD

2.047 .205
COUNTY

ERA ZORI A
NOM- TELLS

FLOR LIFT

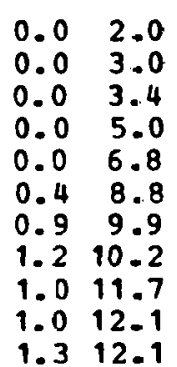

$0.0 \quad 2.0$

$\begin{array}{ll}0.0 & 3.0 \\ 0.0 & 3.4\end{array}$

$0.0 \quad 5.0$

0.06 .8

0.99 .9

$\begin{array}{lll}1.2 & 10.2 \\ 1.0 & 11.7\end{array}$

$\begin{array}{ll}1.0 & 12.1 \\ 1.3 & 12.1\end{array}$

$\begin{array}{rrr}\text { OIL-PRD } & \text { E/RD } & \text { GAS-PRD } \\ & & \\ 4528 & 6.2 & 680 \\ 13917 & 12.7 & 2087 \\ 13892 & 11.1 & 2084 \\ 20044 & 11.0 & 3013 \\ 26591 & 10.7 & 3991 \\ 31996 & 9.5 & 4801 \\ 48884 & 12.4 & 7315 \\ 51838 & 12.5 & 7913 \\ 51366 & 11.1 & 10391 \\ 48080 & 10.1 & 8605 \\ 62377 & 12.8 & 4849\end{array}$


ABANDONED ONSHORE OIL PIELDS NITH OVER 250,000 BBL. CUMULATITE PRODUCTION

DST DISC ABD. PID NITH PIELD NAME

$0.3 \quad 1941 \quad 1970 \quad 67011900$ OLD OCEAN (IARSEN)

DEPTH
10,600

YEAR
1970
1969
1968
1967

DST DISC ABD. FLD NOM FIELD NAME

$03 \quad 1937 \quad 1975 \quad 80700001$ SANDY POINT

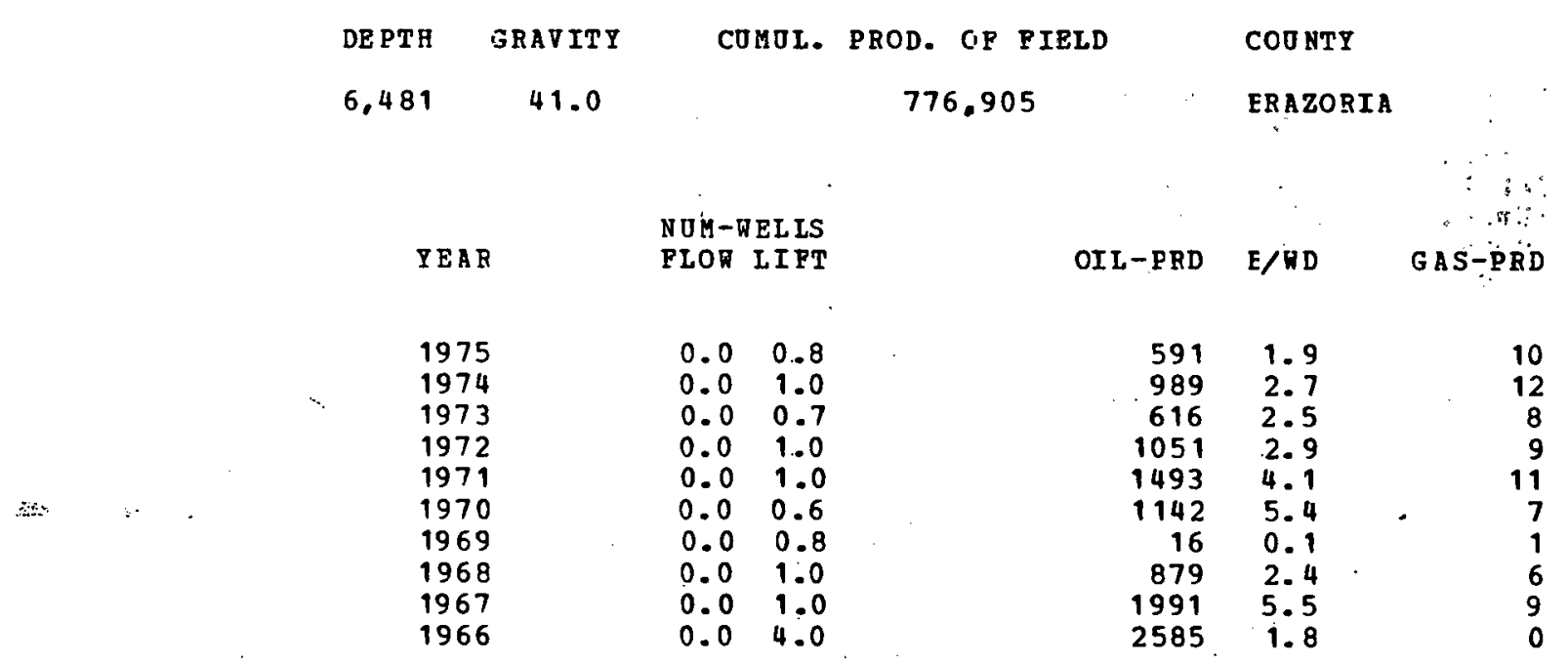

COMOL. PROD. OF FIELD

$1.922,994$

COONTY

ERA 2ORI A
N OU-RELL.LS FIOR IIPT

\section{$0.0 \quad 0.2$}

0.71 .1

$0.0 \quad 1.0$

$\begin{array}{ll}0.0 & 1.0 \\ 0.3 & 0.1\end{array}$ 


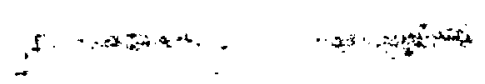

ABANDONED ONSHORE OII FIZLDS RITH OVER 250,000 BBL. CJMULATIVE PRODUCTICN

DST. DISC ABD. FID NOM FIELD NAME

031955196354270750 LOCHRIDGE, NORTH (FRIO 3)

\begin{abstract}
DEPTH
$6,3 \in 2$

GRAVIT $Y$

27.0
\end{abstract}

COMUI. PROD. OF FIELD

716,983

COUNTY

YEAR

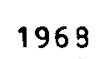

1967

1966

\begin{abstract}
DB PTH
\end{abstract}
7.625

33.7

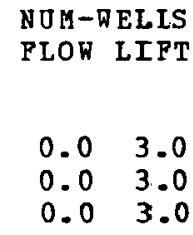

CUMOL. PROD. OP FIEID

$$
670,236
$$

ER A ZOR I A

YEAR
1972
1971
1970
1969
1968
1967
1966

NOM-RELIS

FLOW LIFT

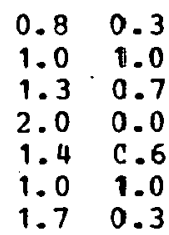

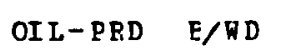

$714 \quad 1.8$

$3420 \quad 4.7$

$9248 \quad 12.7$

2092428.7

$34597 \quad 47.4$

$41312 \quad 56.6$

1746423.9
GAS-PRD

33621

103199

222218

413740

568841

336773

266629 
A3A NDOHED ONSHORE OIL FIELDS NITH OVER 250,000 BBL. CUMULATIVE PRODUCTICN

DST DISC ABD. PID NOY PIELD NAME

031959197517915338 CHOCOLATE BAYOO (GROBBS SAND)
DEPTH GRAVITY

40.8

COMOL. PROD. OP FIELD

669,650

CONNY

ERAZORI A
DST DISC ABD. FLD NDM PIELD NAME

$0319521972 \quad 2882055$ ANGLETON (PRIO 3-A)

\begin{tabular}{lll} 
YEAR & \multicolumn{2}{c}{ NOH-RELIS } \\
& FLON & LIFT \\
1975 & & \\
1974 & 0.0 & 1.0 \\
1973 & 0.0 & 1.8 \\
1972 & 0.0 & 1.3 \\
1971 & 0.0 & 1.3 \\
1970 & 0.0 & 2.0 \\
1969 & 0.0 & 1.0 \\
1958 & 0.6 & 1.4 \\
1967 & 1.0 & 1.0 \\
1966 & 1.0 & 1.0 \\
& 1.0 & 1.0
\end{tabular}

DEPTH GRAVITY

9,452

39.0

COHOI. PROD. OF FIELD

620,142

$\begin{array}{rrr}\text { OIL-PRD } & \text { E/RD } & \text { GAS-PRD } \\ 3262 & 8.9 & 40302 \\ 22953 & 35.9 & 94908 \\ 26187 & 53.8 & 46727 \\ 31110 & 68.2 & 34150 \\ 17170 & 23.5 & 18921 \\ 6996 & 19.2 & 21299 \\ 30819 & 42.2 & 38517 \\ 56071 & 76.8 & 38391 \\ 58120 & 79.6 & 28365 \\ 49394 & 67.7 & 50113\end{array}$

COUNTY

ERAZORIA

\begin{tabular}{lrrrrr} 
IEAR & \multicolumn{2}{c}{ NUE-RELIS } & & \\
& FIOR IIFT & OIL-PRD & E/RD & GAS-PRD \\
1972 & & & & & \\
1971 & 0.0 & 0.8 & 2894 & 9.5 & 8034 \\
1970 & 0.0 & 1.0 & 11898 & 32.6 & 129807 \\
1969 & 0.0 & 0.3 & 5328 & 58.4 & 20443 \\
1968 & 0.7 & 0.0 & 13332 & 54.8 & 147024 \\
1967 & 2.2 & 0.0 & 28588 & 36.1 & 576113 \\
1966 & 3.7 & 0.0 & 55321 & 41.3 & 916595 \\
& 4.0 & 0.0 & 59340 & 40.6 & 736182
\end{tabular}


ABANDONED ONSHORE OIL PIELDS ATTH OVER 250,000 BBL. COMULATIVE PRODUCTION

DST DISC ABD. FLD NOM FIELD NAME

03. 19392882001 ANGLETON

DST DISC ABD. FLD NOH FIELD NAME

031946197096503001 WEST COLOHBIA, DEEP

$\begin{array}{rcccl}\text { DEPTH } & \text { GRAVITY } & \text { COMOI. } & \text { PROD. OF FIELD } & \text { COONTY } \\ 10,500 & 28.0 & & 570,420 & \text { EEAZORIA } \\ \text { DE PTH } & \text { GRAVITY } & \text { COMOI. PROD. OP FIELD } & \text { CODNTY } \\ 6,106 & 33.1 & & 343,144 & \text { EBAZORIA }\end{array}$

6.106

33.1

343,144

EAAZORI A

\begin{tabular}{lll} 
& \multicolumn{3}{l}{ NOR-RELIS } \\
YEAR & PLON & LIFT \\
& & \\
1970 & 0.0 & 0.5 \\
1969 & 0.0 & 1.1 \\
1968 & 0.0 & 1.0 \\
1967 & 0.0 & 1.0 \\
1966 & 0.0 & 1.0
\end{tabular}

$\begin{array}{rrr}\text { OIL-PRD } & \text { E/HD } & \text { GAS-PBD } \\ & & \\ 684 & 3.7 & 2483 \\ 1624 & 4.4 & 8531 \\ 2086 & 5.7 & 8570 \\ 3050 & 8.4 & 8522 \\ 2217 & 6.1 & 6534\end{array}$

DST DISC ABD. FLD NOH FIBLD NAME

031961197222975630 DANBURY DOME (7700 SAND)
CUMUL. PROD. OF PIELD CODNTY

$2 j 2,638$

ERAZORIA

$\begin{array}{cc}\text { DEETH } & \text { GBAVITY } \\ 7.746 & 36.5\end{array}$

7,746

Y B A R
1972
1971
1970
1969
1968
1967
1966

$\begin{array}{lrrrr}\begin{array}{l}\text { NOM-VELIS } \\ \text { PLON LIPT }\end{array} & \text { CI:L-PRD } & \text { E/RD } & \text { GAS-PRD } \\ & & & & \\ 0.0 & 0.7 & 4138 & 17.0 & 1873 \\ 0.0 & 1.0 & 8325 & 22.8 & 22234 \\ 0.0 & 1.0 & 9514 & 26.1 & 15560 \\ 0.0 & 1.0 & 3376 & 9.2 & 5296 \\ 0.0 & 1.7 & 4449 & 7.3 & 4231 \\ 0.0 & 2.0 & 6692 & 9.2 & 14827 \\ 0.4 & 2.3 & 11491 & 11.4 & 29011\end{array}$


ABA RDOARD OASHORE OIL PIELDS RITH OVER 250,000 BBL. COAULATIVE PRODUCTIOA

DST DISC ABD. PID RTI PIELD NA HE

$0319291975 \quad 55036001$ LOST LAKE

DST DISC ABD. FLD NOM PIELD HAME

031944196798085001 MINKIE, NORTE

$\begin{array}{ccccc}\text { DEPTH } & \text { GRATITY } & \text { COAUL. } & \text { PROD. OP FIELD } & \text { COUATY } \\ 2.382 & 20.2 & 1.261 .059 & \text { CBA ABERS }\end{array}$

\begin{tabular}{|c|c|c|c|c|}
\hline YBAR & $\begin{array}{l}\text { עo:-7ELIS } \\
\text { PLOR LIFT }\end{array}$ & OIL - PRD & E/RD D & GA S-PRD \\
\hline $\begin{array}{l}1975 \\
1968 \\
1967 \\
1966\end{array}$ & $\begin{array}{ll}0.0 & 0.0 \\
0.0 & 8.0 \\
0.0 & 8.0 \\
0.0 & 8.0\end{array}$ & $\begin{array}{r}88 \\
1180 \\
7109 \\
5697\end{array}$ & $\begin{array}{l}0.0 \\
0.4 \\
2.4 \\
2.0\end{array}$ & $\begin{array}{r}1 \\
437 \\
2474 \\
2183\end{array}$ \\
\hline
\end{tabular}

DEPTH GRATITY COEOL. PROD. OP PIBLD COOHTY

$\begin{array}{llll}8,925 & 38.0 & 1,223,782 & \text { CBAHBERS }\end{array}$

\begin{tabular}{|c|c|c|c|c|}
\hline YEAR & $\begin{array}{l}\text { NOE-RELIS } \\
\text { FLOR IIFT }\end{array}$ & OIL-PRD & E/प्रD & GAS-PBD \\
\hline $\begin{array}{l}1967 \\
1966\end{array}$ & $\begin{array}{ll}0.1 & 1.3 \\
0.3 & 1.8\end{array}$ & $\begin{array}{l}3198 \\
7296\end{array}$ & $\begin{array}{r}6.2 \\
10.0\end{array}$ & $\begin{array}{r}3902 \\
10565\end{array}$ \\
\hline
\end{tabular}


A BARDORED ORSHORE OIL FIELDS ITH CVER 250,000 BBL C CUOLATIVE PRODUCTIOA

DST DJSC ABD. PLD NOH PIELD NAHE

$031951 \quad 197530824001$ FIG RIDGB, HORTH

\begin{tabular}{|c|c|c|c|}
\hline DE PTH & GRAVIT $Y$ & \multicolumn{2}{|c|}{ COED } \\
\hline 8,198 & 35.7 & & \\
\hline YEAR & & $\begin{array}{l}\text { POA-D } \\
\text { PLOR }\end{array}$ & $\begin{array}{l}\text { ELIS. } \\
\text { LI PT }\end{array}$ \\
\hline $\begin{array}{l}1975 \\
1974 \\
1973 \\
1972 \\
1971 \\
1970 \\
1969 \\
1968 \\
1967 \\
1966\end{array}$ & & $\begin{array}{l}0.0 \\
0.2 \\
0.8 \\
0.6 \\
0.0 \\
0.0 \\
0.2 \\
1.5 \\
2.1 \\
2.6\end{array}$ & $\begin{array}{l}0.1 \\
1.0 \\
1.0 \\
1.01 \\
2.4 \\
3.0 \\
2.8 \\
1.5 \\
0.8 \\
0.4\end{array}$ \\
\hline
\end{tabular}

COUNTI

850,465

CaAabers

$\begin{array}{ccccc}\text { DEPTH } & \text { GRAVITY } & \text { COMO 1. } & \text { PROD. } \text { OF FI ELD } & \text { COOATY } \\ 9.177 & 34.5 & 524.861 & \text { CAA BBERS }\end{array}$

03196419762469500 AYAR OAC, E. (PRIO B-15)

9.177

34.5

524.861

$\begin{array}{rrr}\text { OIL-PRD } & \text { E/RD } & \text { GAS-PRD } \\ & & \\ 377 & 12.4 & 924 \\ 4096 & 9.6 & 10974 \\ 6325 & 9.5 & 17497 \\ 18455 & 30.3 & 26867 \\ 13113 & 14.9 & 39559 \\ 28119 & 25.7 & 54673 \\ 46791 & 42.7 & 61536 \\ 54288 & 49.6 & 83948 \\ 49763 & 46.7 & 77385 \\ 41439 & 37.8 & 59672\end{array}$

\begin{tabular}{|c|c|c|}
\hline YB AR & $\begin{array}{l}\text { YOR- } \\
\text { FLOR }\end{array}$ & $\begin{array}{l}\text { ELIS } \\
\text { LIFT }\end{array}$ \\
\hline $\begin{array}{l}1976 \\
1975 \\
1974 \\
1973 \\
1972 \\
1971 \\
1970 \\
1969 \\
1968 \\
1967 \\
1966\end{array}$ & $\begin{array}{l}0.0 \\
0.0 \\
0.0 \\
0.4 \\
1.3 \\
1.2 \\
0.0 \\
0.1 \\
0.5 \\
1.0 \\
1.0\end{array}$ & $\begin{array}{l}0.8 \\
0.9 \\
1.3 \\
2.7 \\
1.1 \\
1.7 \\
2.0 \\
1.8 \\
0.5 \\
0.0 \\
0.0\end{array}$ \\
\hline
\end{tabular}

$\begin{array}{rrr}\text { OII-PRD } & \text { E/AD } & \text { GAS-PRD } \\ 1227 & 4.0 & 10 \\ 3203 & 9.6 & 11 \\ 1704 & 3.7 & 12 \\ 6216 & 5.4 & 2073 \\ 17097 & 19.4 & 7502 \\ 56387 & 54.5 & 36207 \\ 69372 & 95.0 & 29432 \\ 87806 & 125.5 & 34458 \\ 57589 & 157.8 & 23704 \\ 70280 & 192.5 & 28795 \\ 6.8756 & 188.4 & 31572\end{array}$


ABANDONED ONSHORE OII PIELDS NITH OVER 250,000 BBL. COMOLATIVE PRODUCTICN

DST DISC ABD. FLD NOM FIELD NAHE

0319621974

2468140 ANAHUAC (FB A-2, FRIO 11)

\begin{tabular}{|c|c|c|c|}
\hline DE P TH & GRAVITY & \multicolumn{2}{|c|}{ CU MO L. } \\
\hline 7,542 & 35.9 & & \\
\hline YEAR & & $\begin{array}{l}\text { NOM-n } \\
\text { PIOR }\end{array}$ & $\begin{array}{l}\text { ELIS } \\
\text { IIFT }\end{array}$ \\
\hline $\begin{array}{l}1974 \\
1973 \\
1972 \\
1971 \\
1970 \\
1969 \\
1968 \\
1967 \\
1966\end{array}$ & & $\begin{array}{l}1.2 \\
1.0 \\
0.7 \\
1.0 \\
0.8 \\
0.3 \\
1.4 \\
1.3 \\
1.0\end{array}$ & $\begin{array}{l}0.0 \\
0.5 \\
1.5 \\
0.9 \\
0.3 \\
0.8 \\
0.6 \\
0.7 \\
1.0\end{array}$ \\
\hline
\end{tabular}

\begin{abstract}
DE PTH
GRAVITY
\end{abstract}

6,648

37.3

031943196691960001 TORTLE BAY, EAST

YEAR

1956

\section{NOM-RELIS \\ FLOW LIFT}

$0.0 \quad 0.8$
OIL - P

7880

30999

3809948

38099
22145 31.7

745320.4

$22416 \quad 52.6$

$34719 \quad 47.6$

3416246.8

$29522 \quad 40.4$

COMOL. PROD. OF FIELD

420,874

COUNTY

CHAMBER S
G A S-P RD

240149

510714

209763

138423

34874

56085

94488

111089

34876

$\begin{array}{rr}\text { OIL-PRD } & E / W D \\ 853 & 2.8\end{array}$

GAS-PRD 
ABANDONED ONSHORE OEL FIELDS TITH OVRR 250,000 BBL. CUMULATIVE PRODDCTION

DST DISC ABD. FLD NOM PIELD NAME

03196319752468300 ANAROAC (FB A-4, PRIO 13)

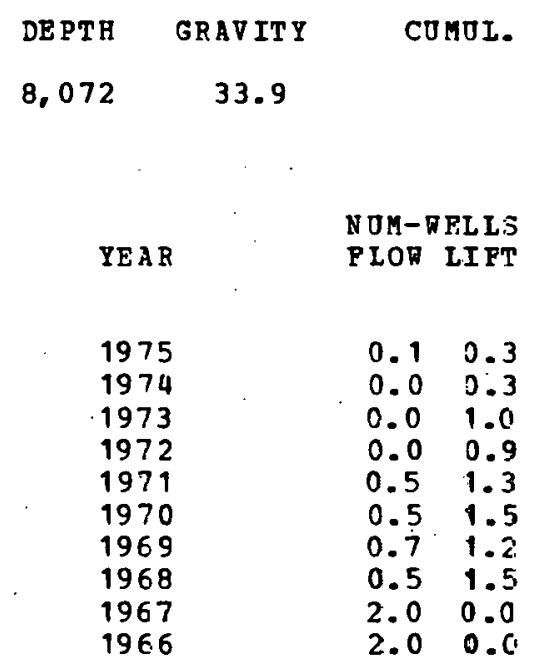

DEPTH

8,088

GRAVITY

37.1
CUHUL. PROD. OF FIELD

404,834

$$
\text { OIL }
$$

OIL-PRD E/HD

$\begin{array}{rr}1398 & 11.5 \\ 598 & 4.9\end{array}$

$\begin{array}{rr}598 & 4.9 \\ 3005 & 8.2\end{array}$

$4046 \quad 12.1$

1218219.1

$23735 \quad 32.5$

$27286 \quad 40.8$

$43388 \quad 59.4$

$\begin{array}{ll}43388 & 59.4 \\ 39041 & 53.5\end{array}$

$32356.44: 3$

COUNTY

CHAHBERS
GA S-PRD

\section{4}

13141

23914

16509

99077

115193

125844

86258

35967

44114

031958197298085075 WINNTE, N. (ENGL IN 8000 SD.)

Y A A R
1972
1971
1970
1969
1968
1967
1966

\section{NOM-VELIS}

FLOW LIFT

$1.6 \quad 0.3$

$2.0 \quad 0.0$

$2.0 \quad 0.0$

$2.0 \quad 0.6$

$3.0 \quad 0.0$

$3.5 \quad 0.4$

\begin{tabular}{rrr} 
OIL-PBD & E/HE & \multicolumn{1}{l}{ GAS-PRD } \\
& & \\
19752 & 29.5 & 67082 \\
30823 & 42.2 & 322359 \\
28918 & 39.6 & 234835 \\
30776 & 3.2 .6$. & 243840 \\
32309 & 29.5 & 421610 \\
32116.5 & 22.5 & 376316 \\
28123 & 19.7 & 373981
\end{tabular}


ABANDONED ONSHORE OIL PIELDS NITH OVER 250,000 BBL. CONOLATIVE PRODUCTION

DST DISC ABD. FLD NDM FIELD NAHE

03196219752468100 ANABOAC (PB A-1, FRIO 13)

DST DISC ABD. FLD NIM PIELD NAMB

031953196998085700 INNIE, N. (KIOCENE 6320)

DEPTH GRAVITT

8,076

34.0

COYOL. PROD. OF PIELD

338,192

$\operatorname{cCONTY}$

CHA GBERS

\begin{tabular}{lll} 
IEAR & \multicolumn{2}{c}{ NOH-RELIS } \\
& FLON & LI PT \\
1975 & & \\
1974 & 0.0 & 0.8 \\
1973 & 0.0 & 1.0 \\
1972 & 0.1 & 0.9 \\
1971 & 1.0 & 0.0 \\
1970 & 0.8 & 0.0 \\
1969 & 1.0 & 0.0 \\
1968 & 1.0 & 0.0 \\
1967 & 1.0 & 0.0 \\
1966 & 1.0 & 0.0 \\
& 1.0 & 0.0
\end{tabular}

$\begin{array}{rrr}\text { OIL-PRD } & \text { E/RD } & \text { GAS-PRD } \\ & & \\ 3197 & 10.5 & 36718 \\ 11942 & 32.7 & 84382 \\ 24580 & 67.3 & 65420 \\ 45390 & 124.4 & 24254 \\ 35257 & 96.6 & 52138 \\ 25441 & 69.7 & 149815 \\ 19452 & 53.3 & 152988 \\ 21696 & 59.4 & 30968 \\ 19524 & 53.5 & 10528 \\ 16463 & 45.1 & 21323\end{array}$

DBPTH GRAVITY

6,321
COHOL. PROD. OF FIELD

310,582
COO NT:Y

CHA MBER S

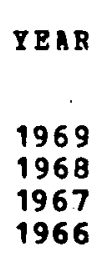

NOH-RELIS PIOD. IIPT

$$
\begin{array}{ll}
0.7 & 0.0 \\
2.0 & 0.0 \\
2.0 & 0.0 \\
2.0 & 0.0
\end{array}
$$

$\begin{array}{rrr}\text { OIL-PRD } & \text { E/ND } & \text { GAS-PRD } \\ & & \\ 2402 & 9.9 & 2957 \\ 30816 & 42.2 & 18585 \\ 34131 & 46.8 & 20854 \\ 27310 & 37.4 & 20764\end{array}$


ABANDONED ONSHORE OIL FIELDS RITH OVER 250,000 BBL. CJMOLATIVE PRODICTION

$\begin{array}{llll}\text { DST } & \text { DTSC ABD. } & \text { PLD NTM PTELD NAME } \\ 03 & 1944 & 1967 & 45423001 \\ \text { JACRSON PASTOR }\end{array}$

DST DISC ABD. FID NOM FIRLD NAME

031945197075235001 BRD FISH REEF, EAST

$\begin{array}{ccccc}\text { DEPTH } & \text { GRAVITY } & \text { COMUL. } & \text { PROD. OP PIELD } & \text { COURTY } \\ 8.128 & 48.0 & 306,216 & \text { CHA GBERS }\end{array}$

\begin{tabular}{|c|c|c|c|c|}
\hline YEAR & $\begin{array}{l}\text { NOG-DELLS } \\
\text { FLOR LIPT }\end{array}$ & OIL-PRD & E/RD & GA S-P RD \\
\hline $\begin{array}{l}1967 \\
1966\end{array}$ & $\begin{array}{ll}0.0 & 0.3 \\
0.0 & 1.0\end{array}$ & $\begin{array}{l}1271 \\
8363\end{array}$ & $\begin{array}{l}10.4 \\
22.9\end{array}$ & $\begin{array}{r}608 \\
2017\end{array}$ \\
\hline
\end{tabular}

\begin{tabular}{|c|c|c|c|}
\hline DEPTH & GRAVITY & COMUL. PROD. OF FIELD & $\operatorname{CoONTY}$ \\
\hline 8,752 & 35.6 & 294,853 & CHAMBERS \\
\hline
\end{tabular}

\begin{tabular}{lll} 
& \multicolumn{2}{c}{ NOE-RELIS } \\
YEAB & PLON IIFT \\
& & \\
1970 & 0.0 & 0.6 \\
1969 & 0.0 & 1.0 \\
1968 & 0.0 & 1.0 \\
1967 & 0.0 & 1.0 \\
1966 & 0.0 & 0.9
\end{tabular}

$\begin{array}{rrr}\text { OII-PRD } & \text { E/RD } & \text { GAS-PBD } \\ & & \\ 999 & 4.7 & 1564 \\ 3513 & 9.6 & 7988 \\ 3510 & 9.6 & 6286 \\ 3062 & 8.4 & 575 \\ 3079 & 9.2 & 5382\end{array}$


ABANDONED ONSHOBE OIL FIELDS WITR OVER 250,000 BBL. CUGOLATIFE PRODUCTION

DST DTSC APD. PLD HOM PIELD NAME

$03 \quad 1964 \quad 1973.96429715$ STORELI (6180)

\begin{tabular}{|c|c|c|c|}
\hline DE PT H & GRAV ITY & \multicolumn{2}{|c|}{ СОМОL. } \\
\hline 6,186 & 38.4 & & \\
\hline YEAR & & $\begin{array}{l}\text { NOM- } \\
\text { FLON }\end{array}$ & $\begin{array}{l}\text { EL IS } \\
\text { LI FT }\end{array}$ \\
\hline $\begin{array}{l}1973 \\
1972 \\
1971 \\
1976 \\
1968 \\
1968 \\
1967 \\
1966\end{array}$ & & $\begin{array}{l}0.1 \\
1.0 \\
1.0 \\
1.9 \\
2.0 \\
2.0 \\
1.9 \\
2.0\end{array}$ & $\begin{array}{l}0.0 \\
0.9 \\
1.0 \\
0.1 \\
0.0 \\
0.0 \\
0.0 \\
0.0\end{array}$ \\
\hline
\end{tabular}

GRAVITY

34.0
CUMU'L. PROD. DF PIELD

262,075
COONTY

CHA MBERS
DE PTH

DST DISC ABD. FLD NII FIELD NAME

031966197525655304 DOJBLE BAYOJ, S. (F-15C, PB 2) 9,600
- YEAR

1975

1974

1973

1972

1971

1970

1969

1967

1966

\section{NOM-RBLIS}

FLOH LIFT

$\begin{array}{ll}0.9 & 0.0 \\ 1.0 & 0.0 \\ 1.0 & 0.0 \\ 1.0 & 0.0 \\ 1.2 & 0.0 \\ 2.0 & 0.0 \\ 2.0 & 0.0 \\ 2.0 & 0.0 \\ 2.0 & 0.0 \\ 1.7 & 0.0\end{array}$

OIL-PRD E/RD

$588 \quad 19$.

1540322.0

22799 31.2

$28796 \quad 39.4$

$32475 \quad 44.5$

$33954 \quad 46.5$

3084244.1

$37987 \quad 52.0$
GA S-PRD

280

17053

27001

60262

31504

29929

34154

34154
57914 
ABANDONED ONSHORE OIL FIELDS NITH OVER 250,000 BBL, CUHULATIVE PRODUCTICN

DST DISC ABD. FLD NUM FIELD NAME

031958197398085150 HINNIE, N. (PB-A, INTMD. SD.)

\section{DEPTH}

8,484
GRAVIT Y

39.1
COMOL. PROD. OP FIELD

259,422
COUNTY

CHAMBERS
YEAR

1973

1968

1967

1966

15 IIELD (S)

\begin{tabular}{|c|c|c|c|c|}
\hline \multicolumn{2}{|c|}{ NUM - WELIS } & \multirow[b]{2}{*}{ OII-PED } & \multirow[b]{2}{*}{$E / W D$} & \multirow[b]{2}{*}{ GAS-PRD } \\
\hline FLOR & L I PT & & & \\
\hline $\begin{array}{l}0.3 \\
0.4 \\
1.4 \\
2.0\end{array}$ & $\begin{array}{l}0.0 \\
0.0 \\
0.03 \\
0.0\end{array}$ & $\begin{array}{r}2071 \\
5874 \\
20679 \\
22429\end{array}$ & $\begin{array}{l}17.0 \\
38.6 \\
34.0 \\
30.7\end{array}$ & $\begin{array}{r}10826 \\
3016 \\
62380 \\
113092\end{array}$ \\
\hline
\end{tabular}

COONTY: CHAMBERS 
ABANDONED ONSHORE OTL FIEIDS MITH OVER 250,000 BBL. COHOLATIVE PRODOCTION

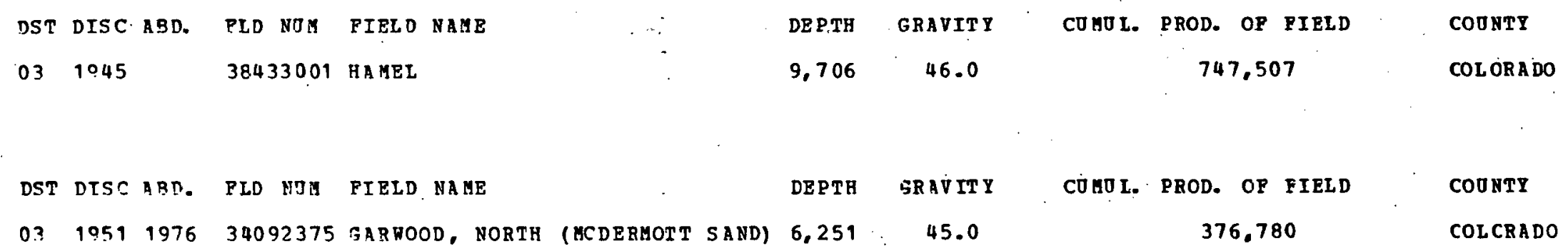

\begin{tabular}{|c|c|c|c|c|c|}
\hline YEAR & $\begin{array}{l}\text { NOM- } \\
\text { FLON }\end{array}$ & $\begin{array}{l}\text { ELIS } \\
\text { LI FT }\end{array}$ & OIL-PRD & $E / R D$ & GA S-P RD \\
\hline $\begin{array}{l}1976 \\
1975 \\
1974 \\
1973 \\
1972 \\
1971 \\
1970 \\
1969 \\
1968 \\
1967 \\
1966\end{array}$ & $\begin{array}{l}0.0 \\
0.0 \\
0.2 \\
0.0 \\
0.0 \\
0.0 \\
0.0 \\
0.0 \\
0.0 \\
0.0 \\
0.0\end{array}$ & $\begin{array}{l}0.8 \\
0.4 \\
0.1 \\
0.3 \\
1.9 \\
2.0 \\
2.0 \\
2.8 \\
3.0 \\
3.0 \\
3.0\end{array}$ & $\begin{array}{r}463 \\
705 \\
914 \\
249 \\
1385 \\
4363 \\
4917 \\
6573 \\
10938 \\
14938 \\
22400\end{array}$ & $\begin{array}{r}1.7 \\
4.6 \\
10.0 \\
2.0 \\
2.0 \\
6.0 \\
6.7 \\
6.4 \\
10.0 \\
13.6 \\
20.5\end{array}$ & $\begin{array}{r}423 \\
637 \\
5515 \\
4 \\
1029 \\
3542 \\
3805 \\
5291 \\
7902 \\
7965 \\
10711\end{array}$ \\
\hline
\end{tabular}


ABANDONED ONSHORE OII PIELDS RITH CVER 250,000 BEL - CJMULATIVE PRODUCTICN

DST DISC ABD. FLD NCM FIELD NAME

031956197119937352 COLTMBOS $(9-8)$

$\begin{array}{ccccc}\text { DEPTH } & \text { GRAVITI } & \text { COKOI. } & \text { EROD. OF FIELD } & \text { CODNTY } \\ 8.795 & 36.9 & 334.713 & \text { COLORADO }\end{array}$

YEAR

NOM-TELLS

1971

1970

1969

1968

1967

1966

3 FIELD (S)
PLOW LIFT

$\begin{array}{ll}0.3 & 0.8 \\ 1.0 & 0.0 \\ 1.0 & 0.0 \\ 1.0 & 0.0 \\ 0.1 & 0.9 \\ 0.0 & 1.5\end{array}$

$\begin{array}{ll}0.3 & 0.8 \\ 1.0 & 0.0\end{array}$

$1.0 \quad 0.0$

0.01 .5
CODNTY: COLORADO
COLORADO

$\begin{array}{rrr}\text { OIL-PRD } & \text { E/RD } & \text { GAS-PBD } \\ & & \\ 6693 & 18.3 & 92539 \\ 5887 & 16.1 & 129066 \\ 9762 & 26.7 & 225800 \\ 17974 & 49.2 & 220542 \\ 18100 & 49.6 & 175049 \\ 15229 & 27.8 & 155263\end{array}$

$1,459,000$ CURUL ATIVE PROD. PROA ABANDONED PIELDS 
ABANDONED ONSHORE OIL PIELDS IITH OVER 250,000 BBL. CDUOLAT IVE PRODDCTION

DST DISC ABD. FID NOH PIELD NAHE

03195119769882250 BIUE RIDGE, N. (VICKSBURG)

\begin{tabular}{|c|c|c|c|c|}
\hline DEPTH & GRAVITI & COMOI. & PROD. OP FIELD & COOATY \\
\hline 7,810 & 28.0 & & $1,610,615$ & FORT BEND \\
\hline
\end{tabular}

TEAR

NOM-RELIS

PLOR LIPT

OIZ-PRD E/MD

GAS-PBD

1976

1975

1974

1973

197

1971

1970

1969

1968

1967

1966

0.00 .3

0.01 .0

$\begin{array}{ll}0.0 & 1.0 \\ 0.0 & 1.0\end{array}$

$0.2 \quad 1.7$

$0.7 \cdot 1.3$

0.02 .0

0.02 .0

0.02 .0

0.02 .0

0.02 .0

$\begin{array}{rrr}582 & 6.4 & 200\end{array}$

$\begin{array}{lll}4273 & 11.7 & 2818\end{array}$

$516214.1 \quad 6780$

$\begin{array}{lll}7022 & 19.2 & 9592\end{array}$

$\begin{array}{lll}11737 & 16.1 & 26272\end{array}$

$\begin{array}{lll}6950 & 9.5 & 18439\end{array}$

$\begin{array}{lll}7557 & 10: 4 & 3221\end{array}$

$\begin{array}{lll}9054 & 12.4 & 5627\end{array}$

63776.7 .6382

$8299 \quad 11.4 \quad 8590$

$\begin{array}{rrr}8299 & 11.4 & 8590 \\ 13543 & 18.6 & 10736\end{array}$

DST DISC ABD. PID NOM PIELD NAME

DEPTH GRAVITY

COMUL. PROD. OP PIELD

CCO RTY

$7,880, \quad 41.0$

856,252

FORT BEND

$\begin{array}{lrrrrr}\text { IEAR } & \begin{array}{r}\text { NOY-RELIS } \\ \text { PLON LIFT }\end{array} & \text { OIL-PRD } & \text { E/RD } & \text { GAS-PRD } \\ & & & & & \\ 1973 & 0.0 & 0.3 & 12 & 0.1 & 22 \\ 1972 & 0.3 & 0.5 & 356 & 1.2 & 2664 \\ 1971 & 0.4 & 0.6 & 1643 & 4.5 & 14769 \\ 1970 & 1.0 & 0.0 & 3875 & 10.6 & 112742 \\ 1969 & 2.3 & 0.1 & 12577 & 14.3 & 424954 \\ 1968 & 3.1 & 1.7 & 31446 & 17.8 & 420280 \\ 1967 & 3.7 & 1.4 & 44428 & 23.9 & 183903 \\ 1966 & 4.8 & 1.2 & 47650 & 21.8 & 93720\end{array}$


ABANDONED ONSHORE OIL PIRLDS NITA OVER 250,000 BBL. COUULATIVE PRODUCTICN

DST DISC ABD. PLD NIB FIBLD NABE

031962197689525775 THOMPSON. (BIOCEN E 12-8 SEG 4)
DE P TH

3,742

Y B A R

1976

1975

1974

1973

1972

1971

1970

1969

1968

1967

1966

DST DISC ABD. FID NTH PIELD NAHE

$\begin{array}{llllllll}03 & 1964 & 1973 & 89525651 & \text { THOHPSON (MIOCENE } & \text { 12-2 SEG 2) }\end{array}$
DEPTH

3,804
COOHTY

FORT BEND
GRAVITY

25.3

CONOI.

$\begin{array}{ll}\text { NOH- RELIS } \\ \text { PLOT LIFT } \\ \\ 0.0 & 0.5 \\ 0.0 & 1.0 \\ 0.1 & 0.9 \\ 0.1 & 1.1 \\ 0.4 & 1.6 \\ 0.0 & 2.0 \\ 0.8 & 1.3 \\ 1.0 & 1.0 \\ 1.0 & 1.0 \\ 1.0 & 1.0 \\ 1.4 & 0.6\end{array}$

350.613

RAVITY

24.2

$\begin{array}{rrr}\text { OII-PRD } & \text { E/RD } & \text { GAS-PRD } \\ & & \\ 7043 & 38.6 & 34781 \\ 21124 & 57.9 & 54485 \\ 26135 & 71.6 & 18377 \\ 5344 & 12.5 & 6959 \\ 27823 & 38.1 & 41828 \\ 37811 & 51.8 & 51229 \\ 42292 & 57.9 & 35590 \\ 29991 & 41.1 & 46423 \\ 24503 & 33.6 & 29684 \\ 19710 & 27.0 & 16048 \\ 16167 & 22.1 & 15009\end{array}$

CONOL. PROD. OP PIELD

311,128

CCORTY

FORT BEM D

$\begin{array}{lrrrrr}\text { YEAR } & \begin{array}{r}\text { BJA-RELIS } \\ \text { PLOR LIPT }\end{array} & \text { OIL-PRD } & \text { E/FD } & \text { GAS-PBD } \\ & & & & & \\ 1973 & 0.0 & 0.1 & 78 & 2.6 & 274 \\ 1972 & 0.0 & 1.5 & 12101 & 22.1 & 6768 \\ 1971 & 0.0 & 2.0 & 24198 & 33.1 & 58163 \\ 1970 & 0.0 & 2.0 & 35443 & 48.6 & 24398 \\ 1969 & 0.0 & 2.0 & 31556 & 43.2 & 15843 \\ 1968 & 0.0 & 2.4 & 27118 & 30.7 & 16436 \\ 1967 & 0.0 & 3.4 & 35840 & 28.7 & 31215 \\ 1966 & 0.4 & 3.6 & 40509 & 27.7 & 25130\end{array}$


ARANDONED ONSHORE OIL PIELDS ITTH OVER 250,000 BBL. COBOLATIVE PRODUCTIOB

DST DISC ABD. PLD YOM FIELD NAME

$03 \quad 19621973 \quad 89525620$ THOHPSON (HIOCENE 12-A LO SEG $4 \quad 3,681$

DE P TH
GRAVITY

25.. 3

\section{Y EAR}

1973

1972

1971

1970

1969

$19 \in 8$

1967

1966

COTNTY: PORT BEND
5 PIELD (S)
COBO I. PROD. OY FIELD

274.188

COONTY

FORT BEKD
ROA-RELIS
PLOR IIPT

0.00 .7

0.02 .0

0.02 .0

0.02 .0

0.02 .0

$0.0 \quad 2.0$

$\begin{array}{ll}0.0 & 2.0 \\ 0.0 & 2.0\end{array}$

$\begin{array}{rrr}\text { OII-PRD } & \text { E/RD } & \text { GAS-PBD } \\ & & \\ 1955 & 8.0 & 1144 \\ 11404 & 15.6 & 11650 \\ 31803 & 43.6 & 40617 \\ 36260 & 49.7 & 18325 \\ 31509 & 43.2 & 18677 \\ 24158 & 33.1 & 12823 \\ 19100 & 26.2 & 7094 \\ 15798 & 21.6 & 5039\end{array}$


ABANDONED ONSHORE OIL PIEINS WITA OVER 250,000 BBL. COHOLATIVE PRODUCTION

DST DISC ABD. FLD NTM PIELD NAHE

$0319401970 \quad 1978001$ ALTA LOHA

DST DISC ABD. PLD NOM PIPLD MAME

$0319541970 \quad 1484564$ ALGOA ( 34 SAND)

$\begin{array}{lcccc}\text { DEPTH } & \text { GRAVITY } & \text { CU.MOI. PROD. OP FIELD } & \text { CODRTY } \\ 9,183 & 37.0 & 1,846,168 & \text { GALVESTON }\end{array}$

YEAR

Q⿻日木-日)

FLOR IIPT

OIL-PRD E/RD

GAS-PRD

1970

1970

1969
1968

1968
1967

1966

$\begin{array}{ll}0.8 & 0.0 \\ 2.6 & 0.0 \\ 3.0 & 0.0 \\ 0.8 & 0.0 \\ 3.0 & 0.0\end{array}$

818
5428

7409
10824

$\begin{array}{ll}10824 & 39.5 \\ 13096 & 12.0\end{array}$

$2.7 \quad 640$

$5.8 \quad .7293$

.8 15733

$\begin{array}{ll}14809 \\ .0 & 20570\end{array}$

\begin{tabular}{|c|c|c|}
\hline DEPTH & GRAVITY & COEOL. PROD. OP FIELD \\
\hline & 34.6 & 359,44 \\
\hline
\end{tabular}

\begin{tabular}{|c|c|c|}
\hline YEAR & $\begin{array}{l}\text { NOH- } \\
\text { PIOR }\end{array}$ & $\begin{array}{l}\text { ELLS } \\
\text { LIFTT }\end{array}$ \\
\hline $\begin{array}{l}1970 \\
1969 \\
1968 \\
1967 \\
1966\end{array}$ & $\begin{array}{l}0.0 \\
0.7 \\
1.0 \\
1.0 \\
1.0\end{array}$ & $\begin{array}{l}0.7 \\
0.3 \\
0.0 \\
0.0 \\
0.0\end{array}$ \\
\hline
\end{tabular}

2 PIEID (S)

$\begin{array}{rrr}\text { OI I-PRD } & \text { E/HD } & \text { GAS-PRD } \\ & & \\ 8520 & 35.0 & 19526 \\ 29428 & 80.6 & 48629 \\ 29929 & 82.0 & 34079 \\ 26195 & 71.8 & 29008 \\ 21868 & 59.9 & 30031\end{array}$


ABANDONED ONSHORE OII FIELDS WITH OVER 250,000 BBL. COMUOLATIVE PRODDCTICN

DST DISC ABD. FLD NOM FIELD NAME

$0319441973 \quad 6757001$ BEECH CREER, NORTH

DST DISC ABD. FID NOM FIEID NAME

$0319471976 \quad 6756001$ BEECH CREER, EAST

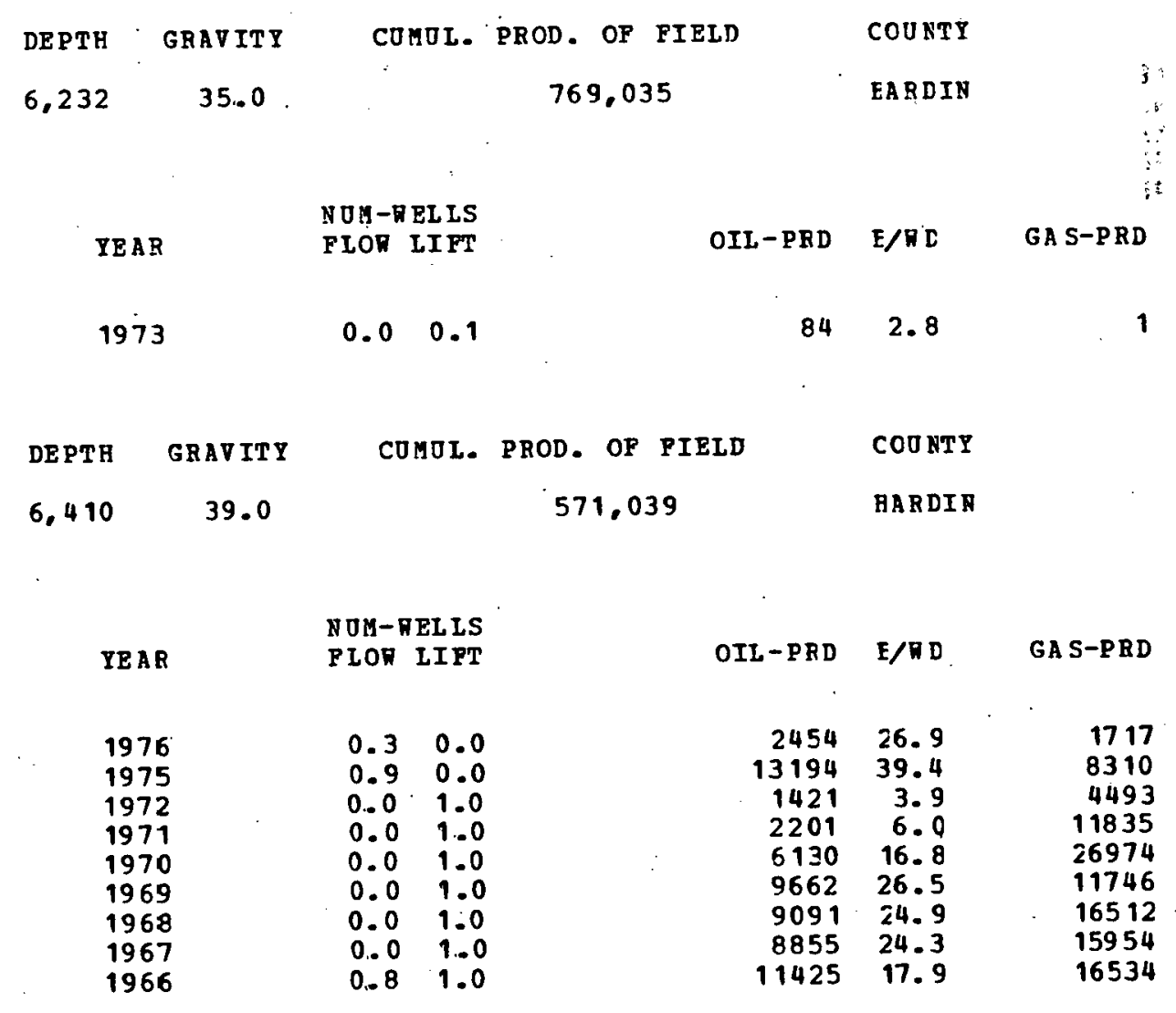


ABANDONED ORSHORE OIL FIELDS RITH OVER 250,000 BBL. COMOLATIVE PRODUCTION

DST DISC ABD. FLD NOM FIELD NAME

$0319451967 \quad 67150001$ OLIVE

$\begin{array}{ccccc}\text { DEPTH } & \text { GRAVITY } & \text { CONOL. } & \text { PROD. OF FIELD } & \text { CODNTY } \\ 10,049 & 44.5 & & 509,170 & \text { EARIIN }\end{array}$

Y E A B

NUH-FELIS
PLON LIFT

1967

1966

3 PIELD (S)

$\begin{array}{rrr}\text { OII-PRD } & \text { E/WD } & \text { GAS-PRD } \\ & & \\ 96 & 0.4 & 479 \\ 821 & 2.2 & 82.59\end{array}$

0.80 .0

$\begin{array}{ll}0.8 & 0.0 \\ 1.0 & 0.0\end{array}$

8212.2

479
82.59 
ABANDONED ONSHORE OIL FIEIDS NITH OVER 250,000 BBL. COMOLATIVE PRODOCTION

DST DISC ABD. FLD NIM FIELD NAME

$03 \quad 19381971 \quad 29996001$ FAIRBANKS

DST DTSC ABD. FLD NTM PIELD NAME

$03 \quad 1929 \quad 1972 \quad 64171001$ MPRAHA

\begin{tabular}{|c|c|c|c|c|}
\hline DEP TH & GRAVITY & COMO I. & PROD. OF FIELD & COONTY \\
\hline 6,836 & 38.5 & & $41,237,925$ & HARRIS \\
\hline
\end{tabular}

YEAR

NOM-WELIS

FLON LIPT

OIL-PRD E/RD

GAS-PRD

1971

1970

1969

1963

1967

1965

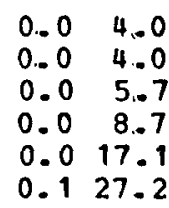

1740

$6543 \quad 4.5$

110245.3

$18610 \quad 5.9$

$26305 \quad 4.2$

$27740 \quad 2.8$

2920
2731
17362
166033
449281
442812

DEPTH GRAVITY

COHO I. PROD. OF FIELD

COUNTY

4.865

27.3

$4,147,689$

HARRIS

YEAR

1972
1971
1970
1969
1968
1967
1966

NOM-VELLS FLOW LIFT

OIL-PRD E/HD

GA S-PRD

$\begin{array}{ll}0.0 & 1.0 \\ 0.0 & 1.0 \\ 0.0 & 1.2 \\ 0.0 & 1.0 \\ 0.0 & 1.5 \\ 0.0 & 4.6 \\ 0.0 & 5.0\end{array}$


ABANDONED ONSHORE OIL FIELDS RITH OVER 250,000 BBI. COMOLATIVE PRODUCTION

DST DISC ABD. FLD NDM FIELD NAME

$0319531972 \quad 7095125$ BENDER (COCKFIELD)

DST DISC ASD. PID NOM PIELD NAME

$03 \quad 19371973 \quad 18992001$ NLINTON

\begin{tabular}{|c|c|c|c|}
\hline DEPTH & GRAVITY & \multicolumn{2}{|c|}{ COMUL. } \\
\hline 5,811 & 39.0 & & \\
\hline YEAB & & NOH- & ELIS \\
\hline $\begin{array}{l}1972 \\
1971 \\
197 \mathrm{C} \\
1969 \\
1968 \\
1967 \\
1966\end{array}$ & & $\begin{array}{l}0.0 \\
0.0 \\
0.0 \\
0.0 \\
0.0 \\
0.0 \\
0.7\end{array}$ & $\begin{array}{r}2.5 \\
7.8 \\
5.5 \\
9.0 \\
10.8 \\
13.0 \\
17.0\end{array}$ \\
\hline
\end{tabular}

PROD. OP PIELD

$3,793,190$

$\cos \operatorname{sT}$

HARBIS

$\begin{array}{rrr}\text { OIL-PRD } & \text { E/RD } & \text { GAS-PED } \\ & & \\ 7638 & 8.4 & 44553 \\ 30110 & 10.6 & 190982 \\ 45830 & 13.2 & 130847 \\ 86308 & 26.3 & 155707 \\ 98172 & 24.8 & 79587 \\ 1138427 & 29.2 & 104386 \\ 252211 & 39.1 & 52972\end{array}$

DEPTH GRAYTIT

COYOL. PROD. OF FIELD

$1,691,997$

COUNTI

HAREIS
6,500

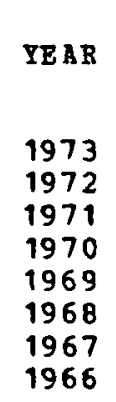

NOH-VELIS

PLON LIFT

$0.0 \quad 0.6$

0.03 .8

0.04 .0

0.04 .0

0.14 .7

$1.24 ! 1$

$\begin{array}{ll}1.0 & 2.6 \\ 1.0 & 5.3\end{array}$

$\begin{array}{rrr}\text { OIL-PRD } & \text { E/RE } & \text { GAS-PRD } \\ & & \\ 2480 & 11.6 & 7 \\ 5717 & 4.1 & 176 \\ 6558 & 4.5 & 2817 \\ 10354 & 7.1 & 4127 \\ 16783 & 9.7 & 12754 \\ 21677 & 11.3 & 9066 \\ 29154 & 22.3 & 9689 \\ 40644 & 97.8 & 10501\end{array}$


ABANDONED ONSHORE OIL FIELDS RITH OVER 250,000 BBL. COHOLATEVE PRODOCTION

DST DISC ABD. PLD NOM PIEID NAME

031939197642905001 HOOSTON, NORTA
DE PTH GBAV IT I

9,035

37.4

YE A B

1976

1975

1974

1973

1972

1971
1970

1970
1969

1968

1967

1966

$$
\begin{aligned}
& \text { NOH- RELIS } \\
& \text { PLOR LI FT }
\end{aligned}
$$

PIOR LIFT

$\begin{array}{ll}0.0 & 0.8 \\ 0.0 & 1.0 \\ 0.0 & 1.0 \\ 0.0 & 0.8 \\ 0.0 & 1.0 \\ 0.0 & 1.0 \\ 0.0 & 1.8 \\ 0.0 & 2.0 \\ 0.0 & 1.9 \\ 0.1 & 1.4 \\ 0.9 & 2.0\end{array}$

$0.0 \quad 0.8$

0.01 .0

$0.0 \quad 0.8$

0.01 .0

$0.0 \quad 1.8$

0.01 .9

0.92 .0

COU RTY

HAREIS

$$
1,607,665
$$

OII-PRD E/RD GAS-PRD

DEPTH GRAVITT

COMUL. PROD. OF FIELD

$\begin{array}{rrr}1888 & 6.2 & 591 \\ 2921 & 8.0 & 910 \\ 3234 & 8.9 & 972 \\ 3970 & 13.1 & 3628 \\ 7775 & 21.3 & 7223 \\ 9698 & 26.6 & 8923 \\ 8263 & 12.9 & 8466 \\ 8224 & 11.3 & 10813 \\ 10046 & 14.4 & 9990 \\ 10656 & 19.5 & 11621 \\ 16739 & 15.7 & 18721\end{array}$

DST DISC ABD. FLD NOA PIELD NAHE

03195519681285600 ALCO-MAG, DEST (YEGOA)

8,056

40.4

$$
1,026,153
$$

\begin{tabular}{|c|c|c|c|c|}
\hline YEAR & $\begin{array}{l}\text { NOA-NELIS } \\
\text { PLOR LIPT }\end{array}$ & OIL-PRD & $E / W D$ & GAS-PRD \\
\hline $\begin{array}{l}1968 \\
1967 \\
1966\end{array}$ & $\begin{array}{ll}2.5 & 0.0 \\
6.0 & 0.0 \\
6.0 & 0.0\end{array}$ & $\begin{array}{r}7873 \\
16574 \\
35571\end{array}$ & $\begin{array}{r}8.6 \\
7.6 \\
16.2\end{array}$ & $\begin{array}{r}26095 \\
60610 \\
156980\end{array}$ \\
\hline
\end{tabular}

CODNTY

EARRIS 
ABANDONED ONSHORE OIL FIELDS WITH OVER 250,000 BBL. COGOLATIVE PRODOCTION

DST NISC ABD. . PLD NDY FIELD NAME

$03.1948197 .50366001 \mathrm{KOHL}$ HAN

\begin{tabular}{|c|c|c|c|}
\hline DEPTH & GRAV IT $Y$ & \multicolumn{2}{|c|}{ COHOL. } \\
\hline 5,876 & 38.0 & & \\
\hline TEAR & & $\begin{array}{l}\text { YOX- } \\
\text { FLOR }\end{array}$ & $\begin{array}{l}\text { EL LS } \\
\text { BI FT }\end{array}$ \\
\hline $\begin{array}{l}1971 \\
1970 \\
1969 \\
1968 \\
1967 \\
1966\end{array}$ & & $\begin{array}{l}0.1 \\
0.0 \\
0.2 \\
0.0 \\
0.6 \\
2.0\end{array}$ & $\begin{array}{l}4.7 \\
5.0 \\
4.7 \\
5.0 \\
2.9 \\
3.0\end{array}$ \\
\hline
\end{tabular}

DEPTH GRAVITY
5,850

37.0

03. 1954197390626500 TOMBALl, SE. (COCKPIELD PIRST)

YEAR

1973
1972
1971
1970
1969
1968
1967
1966

\section{PLOR IIFT}

$\begin{array}{ll}0.1 & 0.0 \\ 3.2 & 2.0 \\ 6.0 & 2.2 \\ 6.8 & 3.2 \\ 3.4 & 6.6 \\ 8.3 & 2.6 \\ 6.5 & 4.5 \\ 4.0 & 7.0\end{array}$

$1,023,834$

COONTY

HAR FIS 
ABANDONED ONSHORE OIL FIELDS RITH CVER 250,000 BBL. COMULATIVE PHODUCTICN

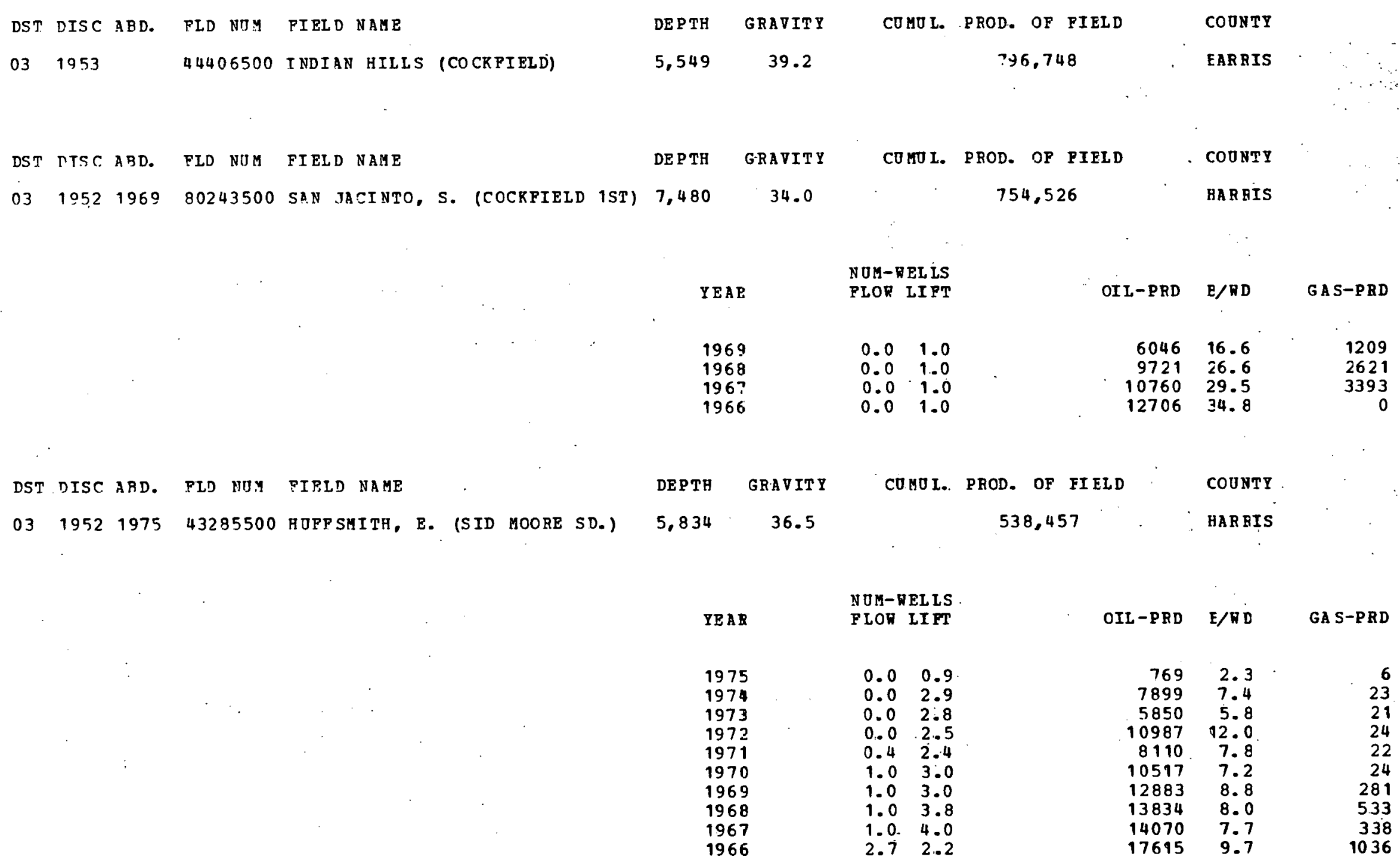


ABANDONED ONSHORE OIL FIEIDS MITH OVER 250,000 BBL. COHOLATIVE FRODOCTION

DST DISC ABD. FLD NTM FIELD NAME

$03 \quad 1944 \quad 1958 \quad 85306001$ SPRING

DST DISC ABC. FLD NIM FIELD NAME

n3 1952197229991400 FAIRBANKS, S. (FAIRBANKS SAND)

$\begin{array}{cc}\text { DEPTH } & \text { GRAVITI } \\ 6,249 & 38.0 \\ & \\ \text { DEPTH } & \text { GRAVITY } \\ 7.030 & 33.0\end{array}$

7,030
COMUL. PROD. OP PIELD

493,864

COMUL. PROD. OP FIELD

407,859

$\begin{array}{rr}\text { OIL-PRD } & \text { E/RD } \\ & \\ 1338 & 7.3 \\ 4435 & 12.2 \\ 5933 & 16.3 \\ 5770 & 15.8 \\ 6873 & 18.8 \\ 7829 & 21.4 \\ 8238 & 22.6\end{array}$

$0.0 \quad 0 . .5$

0.01 .0

$0.0 \quad 1.0$

0.01 .0

0.01 .0

$\begin{array}{ll}0.0 & 1.0 \\ 0.0 & 1.0\end{array}$

1968

1966

DST DISC ABD. FLD Y़IOM FIEID NAME

$03 \quad 1954 \quad 1973$

1279913 ALCO-MAG (8000)
339,872 HARRIS

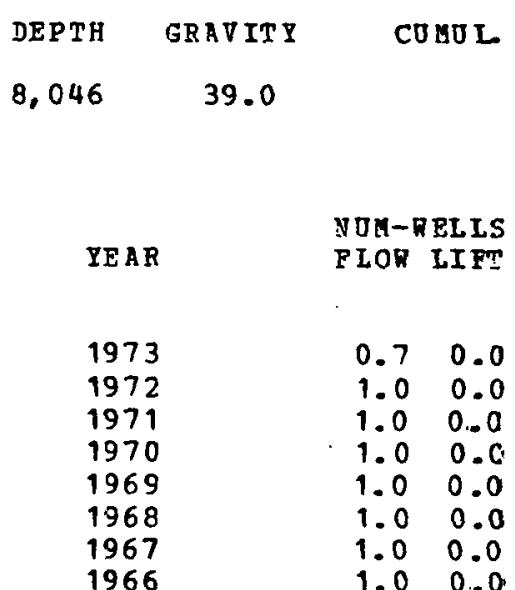

COUNTI

HARRIS

COONTY

EARRIS

\section{GA S-PRD \\ 127


ABANDONED ONSHORE OIL FIELDS ITH OVER 250,000 BBL. COAOLATIVB PRODOCTION

DST DISC ABD. PLD NOH FIELD NAME

031956196795731750 REBSTER (VICKSBORG 7650 SD.)

DST DISC ABD. FID BOH FIBLD NAME

031965197318992624 CLIHTON (VICKSBORG 1)

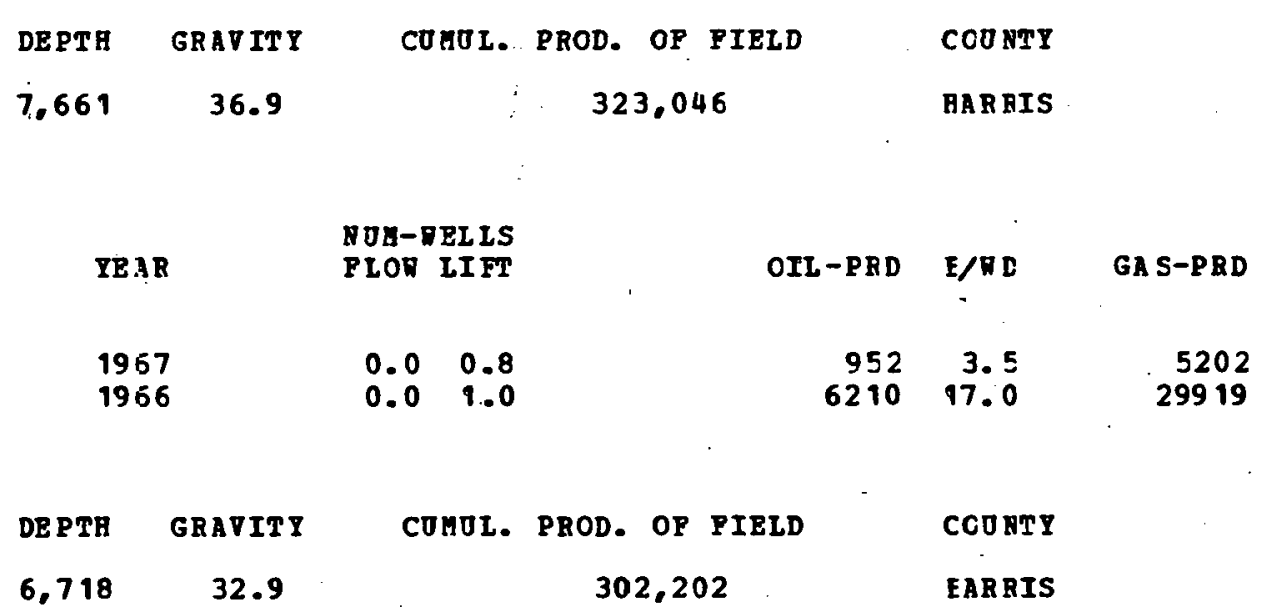

\begin{tabular}{lll} 
& \multicolumn{2}{c}{ NOA-HELIS } \\
PEAR & PLON LIFT \\
& & \\
1973 & 0.5 & 0.0 \\
1972 & 1.4 & 0.4 \\
1971 & 2.0 & 1.0 \\
1970 & 2.0 & 1.0 \\
1969 & 2.8 & 0.3 \\
1968 & 2.9 & 0.0 \\
1967 & 0.7 & 0.0 \\
1966 & 1.3 & 0.0
\end{tabular}


ABANDOEED ONSHORE OIL ZIELDS ITTB OVBR 250,000 BBL. CUBULATIVE PRODUCTICN

DST DISC ABD. FID NOM FIBLD MALE

031939

1327001 ALDINE

DST DISC ABD. PLD ROG FIELD NAHE

$0319551973 \quad 5388360$ BA HAEL (6470, DRYER)

\begin{tabular}{|c|c|c|c|c|}
\hline DE P TH & GRAVITI & CO $80 \mathrm{I}$. & PROD. OP FIELD & COONTY \\
\hline 6,824 & 37.0 & & 283,676 & EARRIS \\
\hline DEPTH & GRAVITY & CONOI. & PROD. OP FIELD & COONTY \\
\hline 6,472 & 33.0 & & 273,468 & BARAIS \\
\hline
\end{tabular}

$\begin{array}{lll} & \text { ROL- } & \text { RELIS } \\ \text { YEAR } & \text { PLON } & \text { LI PT } \\ & & \\ 1973 & 0.0 & 1.1 \\ 1972 & 0.0 & 2.0 \\ 1971 & 0.0 & 2.0 \\ 1970 & 0.0 & 2.0 \\ 1969 & 0.0 & 2.0 \\ 1968 & 0.0 & 2.0 \\ 1967 & 0.0 & 2.0 \\ 1966 & 2.0 & 0.0\end{array}$

DEPTB GRATITI

CUMOL. PROD. OP FIELD

COONTY
OIL-PRD E/ND

$\begin{array}{lll}1744 & 4.4 & 13586 \\ 3618 & 5.0 & 16244\end{array}$

$3618 \quad 5.0 \quad 16244$

$5308 \quad 7.3 \quad 17645$

$\begin{array}{lll}6141 & 8.4 & 24723\end{array}$

$803411.0 \quad 25497$

$9680 \quad 13.3 \quad 23243$

$\begin{array}{lll}15845 & 21.7 & 31396\end{array}$
DST DISC ABD. FID NOA PIELD MBE

031951
16533800 CEDAR PAYOU, H. (HUCKELEOT)
6,316

30.0
BAREIS 
ABANDONED ONSHORE OIL FIELDS NITH OVER 250,000 BBL. COHOLATIVE PRODUCTION

\begin{abstract}
DST DISC ABD. PLD NIM PIELD NAHE
031948

COTNTY: HARRIS
\end{abstract}

$\begin{array}{ccccc}\text { DEPTR } & \text { GRAVITY } & \text { COHOL. } & \text { PBOD. OF FIELD } & \text { CONHTY } \\ 7,082 & 35.6 & 259,385 & \text { EARRIS }\end{array}$

20 PIEID (S)
$60,35 C, 123$ CONOLATIVE PROD. PROB ABANDONED RIELDS 
ABA NDONED ONSHORE OII FIELDS NITH OVER 250,000 BBI. COHOLATIVE PRODOCTIOR

DST DISC ABD. FID NOA FIELD NABE

$\begin{array}{llllll}03 & 1957 & 1973 \quad 34302250 \text { GEBHART (YEGOA 3-D) }\end{array}$

NST DISC ABD. FID NOM PIRLD NAME

$0319591973 \quad 34302500$ GEBHART (YEGTA 4-A)

$\begin{array}{ccccc}\text { DEPTH } & \text { GRAVITY } & \text { COUOL. PROD. OF FIELD } & \text { CCOETY } \\ 6,382 & 41.0 & 926,197 & \text { JASEER }\end{array}$

TE AR

$$
\begin{aligned}
& \text { NOA-RELLS } \\
& \text { FLOR IIFT }
\end{aligned}
$$

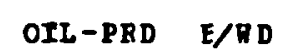

1968

1968
1967

1966

$$
\begin{array}{ll}
0.0 & 0.9 \\
0.0 & 2.0 \\
0.0 & 2.0 \\
0.0 & 1.5 \\
0.0 & 2.3 \\
0.2 & 3.0 \\
1.0 & 3.7 \\
2.8 & 3.2
\end{array}
$$

926,197

JAS EER

$\begin{array}{cccc}\text { DEPTH } & \text { GRAVITY } & \text { COLOL. PROD. OP FIELD } & \text { CODRTY } \\ 6,380 & 39.9 & 760,804 & \text { JASPER }\end{array}$

IEAR

1973
1972
1971
1970
1969
1968
1967
1966

ROA-NELIS PLON IIPT

$$
\begin{array}{ll}
0.0 & 0.6 \\
0.0 & 1.0 \\
0.0 & 1.0 \\
0.0 & 2.0 \\
0.0 & 2.0 \\
0.3 & 1.7 \\
2.0 & 0.07 \\
2.0 & 0.3
\end{array}
$$

$\begin{array}{rrr}\text { OIL-PRD } & \text { E/AD } & \text { GAS-PRD } \\ & & \\ 1312 & 6.2 & 4092 \\ 3169 & 8.7 & 8043 \\ 6952 & 19.0 & 2807 \\ 22947 & 34.4 & 4824 \\ 27295 & 37.4 & 17652 \\ 29411 & 40.3 & 22416 \\ 32973 & 43.4 & 31917 \\ 32719 & 29.9 & 29935\end{array}$


ABANDONED ONSHORE OIL FIELDS NITH OVER 250,000 BBL. COMOLATIUE PRODOCTION

DST DISC ABD. FLD NOM FIELD NAME

03. 1959197216846566 CHAMPION (NILCOX 9050)

\begin{tabular}{|c|c|c|c|c|}
\hline DEPTH & GRAVIT Y & CUMOL. & PROD. OF FIELD & CCONTY \\
\hline 9,050 & 45.1 & & 458,643 & JAS EER \\
\hline
\end{tabular}

YEAR

NOM- MELLS

OIL-PRD E/WD

GA S-PRD

1972

1971

1970

1969

1968

1967

1966

COONTY: JASPRR

3 FIE ID (S)

$\begin{array}{rrrrr}0.0 & 3.0 & 4870 & 4.4 & 17631 \\ 0.0 & 2.2 & 14143 & 17.9 & 20334 \\ 0.0 & 2.0 & 20971 & 28.7 & 21773 \\ 0.0 & 2.0 & 24666 & 33.8 & 25679 \\ 0.3 & 2.5 & 23818 & 23.7 & 31415 \\ 1.0 & 2.0 & 25732 & 23.5 & 41801 \\ 1.8 & 2.0 & 25223 & 18.4 & \Xi 5246\end{array}$

2,145,644 COMOLATIVE PROD. FROA ABANDONED PIEIDS 
ABANDONED ONSHORE OII FIEIDS NITH OVER 250,000 BBL. CJHOLATIVE PRODOCTION

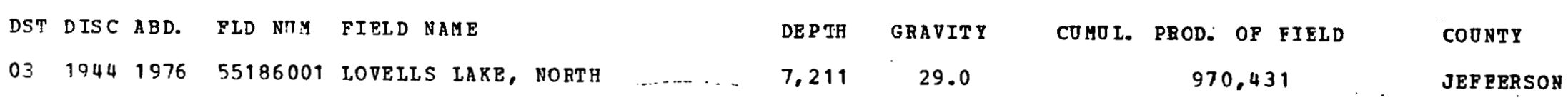

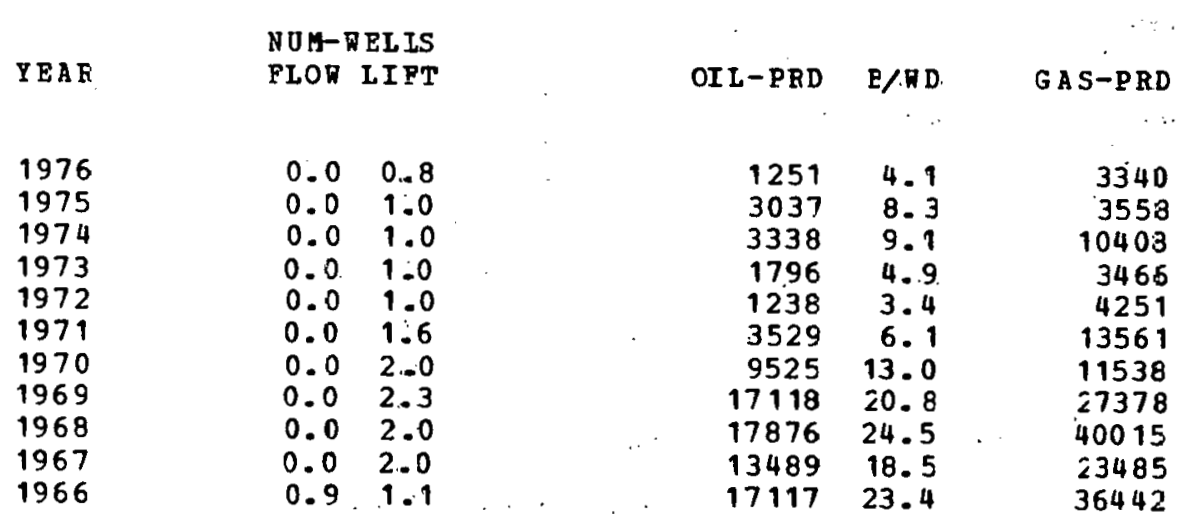

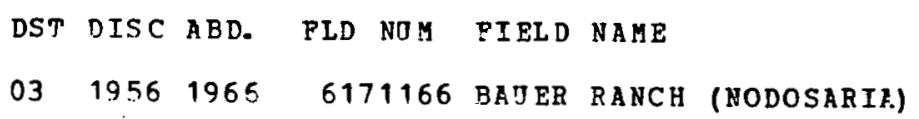


ABANDONED ONSHORE OIL FIELDS UITH OVZR 250,000 BBL. COUDLAT IVE PRODUCTION

DST DISC ABD. PLD MOH FIEID NAME

$031961 \quad 1967 \quad 7988852$ BIG BILL, N⿴. (8900)

DST DISC ABD. PLD NOA FIELD HABE

03.196119742315672 AGBLIA (BACKBERRY $\$ 2$ )

$\begin{array}{ccccc}\text { DEPTE } & \text { GRAVITY } & \text { COYJL. } & \text { PROD. OF PIELD } & \text { COD RTY } \\ 8,984 & 37.1 & 400,945 & \text { JEPPERSON }\end{array}$

\begin{tabular}{|c|c|c|c|c|}
\hline YEAR & $\begin{array}{l}\text { NOM-RELIS } \\
\text { PLOD LIFT }\end{array}$ & OIL -PRD & $E / D D$ & GA S-PRD \\
\hline $\begin{array}{l}1967 \\
1966\end{array}$ & $\begin{array}{ll}0.0 & 1.0 \\
0.0 & 1.5\end{array}$ & $\begin{array}{l}3326 \\
7521\end{array}$ & $\begin{array}{r}9.1 \\
13.7\end{array}$ & $\begin{array}{r}6379 \\
48347\end{array}$ \\
\hline
\end{tabular}

$\begin{array}{ccccc}\text { DEPTE } & \text { SRAVITY } & \text { COHOL. PROD. OP FIELD } & \text { COURTY } \\ 7,438 & 39.8 & 370,314 & \text { JEPEERSON }\end{array}$

$\begin{array}{lrrrrr}\text { YEAE } & \begin{array}{r}\text { ROB-RELIS } \\ \text { PLOD LIFT }\end{array} & \text { OIL-PBD } & \text { E/RD } & \text { GAS-PBD } \\ & & & & & \\ 1974 & 0.0 & 0.8 & 1550 & 5.1 & 14047 \\ 1973 & 0.0 & 0.3 & 439 & 4.8 & 2747 \\ 1972 & 0.0 & 1.0 & 2488 & 6.8 & 6198 \\ 1971 & 0.0 & 1.0 & 1717 & 4.7 & 1263 \\ 1970 & 0.0 & 1.0 & 2333 & 6.4 & 14756 \\ 1969 & 0.0 & 1.0 & 3046 & 8.3 & 14101 \\ 1963 & 0.0 & 1.0 & 4299 & 11.8 & 10141 \\ 1967 & 0.0 & 2.0 & 6837 & 9.4 & 13483 \\ 1965 & 0.1 & 3.2 & 17308 & 14.6 & 45113\end{array}$


ABANDÓNED ONSHORE OIL PIELDS HITH OVER 250,000 BBL. COMOLATIVE PRODOCTION

DST DISC ABD. FID VIM FIELD NAME

$03 \quad 19691976$

2315.964 AMELIA (MABGINDIINA 4)
DEPTH

6,284

GRAV ITY

28.9

CUMOL. PROD. OF FIELD

368,173

COUNTY

JEPFERSON
DST DISC ABD. FID HDM FIBLD NAME

0319601967

2315608 AMELIA. (HACRBERRT \#1)

$\begin{array}{rrr}\text { OIL-PRD } & \text { E/HD } & \text { GAS-PRD } \\ & & \\ 416 & 3.4 & 101 \\ 2302 & 6.3 & 778 \\ 5137 & 7.7 & 11361 \\ 5946 & 8.1 & 4262 \\ 11072 & 12.6 & 5777 \\ 7344 & 11.0 & 2480 \\ 10742 & 29.4 & 5147 \\ 15016 & 23.5 & 8860 \\ 19675 & 29.4 & 18126 \\ 23104 & 31.6 & .16165 \\ 20876 & 28.6 & 12690\end{array}$

DEPTH GRAVITY

7,349

38.7

COMOL. PROD. OP FIELD

327.855

COONTY

JEPFERSON

\begin{tabular}{|c|c|c|c|c|}
\hline YEAR & $\begin{array}{l}\text { NOM- } \text { RELIS } \\
\text { FLON LIFT }\end{array}$ & OIL - PRD & $E / R C$ & GA S-PRD \\
\hline $\begin{array}{l}1967 \\
1966\end{array}$ & $\begin{array}{ll}0.7 & 0.3 \\
1.0 & 0.00\end{array}$ & $\begin{array}{l}3770 \\
5558\end{array}$ & $\begin{array}{c}11.3 \\
15.2\end{array}$ & $\begin{array}{r}11991 \\
0\end{array}$ \\
\hline
\end{tabular}


ABANDONED ONSHORE OIL FIEIDS WITH OVER 250,000 BBL. COUULATITE FRODUCTION

DST DISC ABD. PLD NOM PIELD NAME

$0319621970 \quad 86429897$ STONELI $\quad(7150)$

DEPTH
7,158

YEAE
$197 C$
$196 C$
1968
1967
1966

7 PIEID (S)

(2)

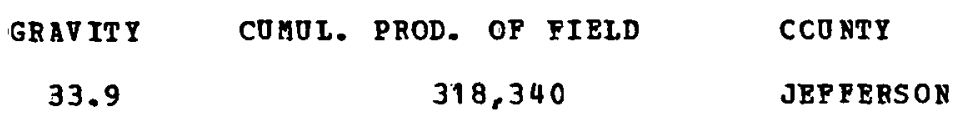

$\begin{array}{lrrrr}\begin{array}{l}\text { ROM-WELIS } \\ \text { PION LIFT }\end{array} & \text { OIL PRD } & \text { E/RD } & \text { GAS-PRD } \\ & & & & \\ 0.0 & 1.0 & 5463 & 15.0 & 1565 \\ 0.0 & 1.0 & 19731 & 54.1 & 9605 \\ 0.0 & 1.8 & 17815 & 27.9 & 12502 \\ 0.8 & 1.3 & 34340 & 45.2 & 20000 \\ 1.3 & 0.7 & 29179 & 40.0 & 13284\end{array}$

\section{COUNT: JEPPERSON}

$3,191,847$ CUMOLATIVE PROD. PROA ABANDONED PIELDS 
ABANDONED ONSHORE OIL FIELDS NITH OVER 250,000 BBL. CUMOLATIVE FRODDCTION

DST DISC ABD. PID NUM FIELD NAME

031954197160561625 MER $A$ HANT, NE. (EY-1A PB 1). $\begin{array}{cc}\text { DEPTH } & \text { GRAVITY } \\ 9,150 & 32.6\end{array}$

COMOL. PROD. OF FIELD

524,975

CODNTT

LI BERTY

$\begin{array}{lll} & \text { NOH-RELIS } \\ \text { YEAR } & \text { PLOR } & \text { IIFT } \\ & & \\ 1971 . & 0.0 & 0.6 \\ 1970 & 0.0 & 1.0 \\ 1969 & 0.1 & 0.9 \\ 1968 & 0.0 & 1.0 \\ 1967 & 0.0 & 1.0 \\ 1966 & 0.0 & 1.0\end{array}$

$\begin{array}{rr}\text { OIL-PAD } & E / H D \\ & \\ 398 & 1.9 \\ 3389 & 9.3 \\ 4164 & 11.4 \\ 3921 & 10.7 \\ 4761 & 13.0 \\ 4082 & 11.2\end{array}$

GAS-PBD

1.9
9.3

11.4

13.0

4082 1. 2

5
12
12
11
12
0

DST DISC ABD. PID NOA PIELD NAUE

DBPTH GRAVITY

COHOL. PROD. OP FIELD

COONTY

03.1954

18929200 CLEVELAND (COCRPIELD A, NE SES) 5,894

42.0

355,576

IIBEBTY

DST DISC ARD. FLD NOM PIELD NAHE

DEPTH GRAVITT

COMOL. PROD. OP PIELD

CONTY

031954196760561250 MERCHANT, NE. (EY-1)

32.0

349,227

I IB ERTY

\begin{tabular}{|c|c|c|c|c|}
\hline YEAR & $\begin{array}{l}\text { NOH-RELLS } \\
\text { PION LIFT }\end{array}$ & OIL-PRD & $E / R I$ & GA S-P RD \\
\hline $\begin{array}{l}1967 \\
1966\end{array}$ & $\begin{array}{ll}0.0 & 0.4 \\
0.0 & 1.0\end{array}$ & $\begin{array}{r}6230 \\
17234\end{array}$ & $\begin{array}{l}41.0 \\
47.2\end{array}$ & $\begin{array}{l}2645 \\
6562\end{array}$ \\
\hline
\end{tabular}


ABANDONED ONSHORE OIL FIELDS WITH OVER 250,000 BBL. CUMULAT IVE PRODUCTION

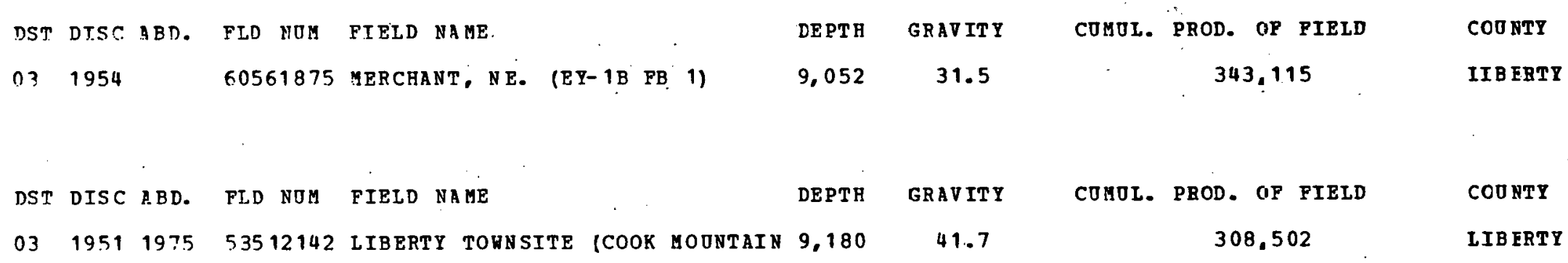

\begin{tabular}{lll} 
& \multicolumn{2}{l}{ NOB-WELIS } \\
YEAR & PLOW LIFT \\
& & \\
1975 & 0.0 & 0.2 \\
1974 & 0.0 & 0.6 \\
1973 & 0.0 & 0.8 \\
1972 & 0.0 & 1.0 \\
1971 & 0.0 & 1.0 \\
1970 & 0.0 & 1.0 \\
1969 & 0.0 & 1.0 \\
1968 & 0.0 & 1.0 \\
1967 & 0.0 & 1.0 \\
1966 & 0.0 & 1.0
\end{tabular}

$\begin{array}{rrr}\text { OIL-PRD } & \text { E/HD } & \text { GAS-PRD } \\ & & \\ 761 & 12.5 & 2 \\ 319 & 1.5 & 8 \\ 2590 & 9.5 & 116 \\ 3664 & 10.0 & 1779 \\ 3172 & 8.7 & 1589 \\ 4003 & 11.0 & 1900 \\ 2548 & 7.0 & 1276 \\ 2312 & 6.3 & 1170 \\ 2689 & 7.4 & 1346 \\ 2393 & 6.6 & 1199\end{array}$

COTNTY: LIBERTY 
ABA NDONED ONSHOBE OII PIZLDS ITH OVER 250,000 BRL. COMOLATIVE PRODOCTICN

DST DISC ABD. FLD NOM PIELD NAME

031946197361048001 MIDPIELDS

DST DISC ABD. FID NOM FIELD NAME

$031946 \quad 1970 \quad 89826001$ TIDEHAVEN

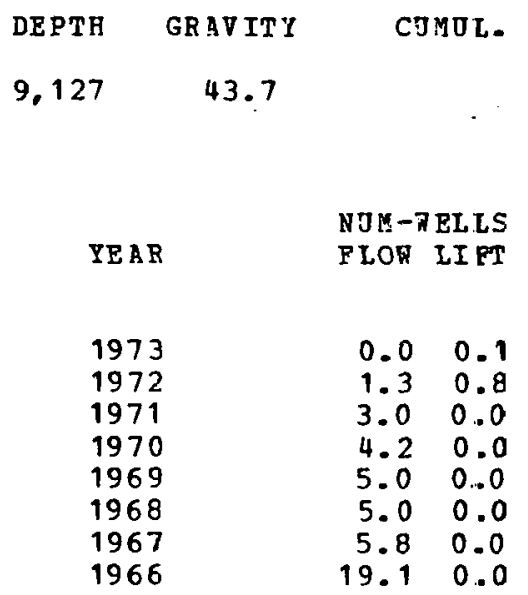

DEPTH.

GRAVITY

8,468

41.4

COMOI. PROD. OF FIELD

OIL-PRD

$\mathrm{E} / \mathrm{B}$

GA S-PRD

$7.684,062$

COONTY

YAT AGORDA

$\begin{array}{rrr}24 & 0.8 & 12 \\ 5714 & 7.5 & 231925 \\ 17552 & 16.0 & 668844 \\ 29746 & 19.6 & 1029289 \\ 13602 & 7.5 & 374090 \\ 33625 & 18.4 & 779539 \\ 62233 & 29.7 & 1376459 \\ 185354 & 26.6 & 4383605\end{array}$

$2,745,916$

CORNTY

MAT AGORDA

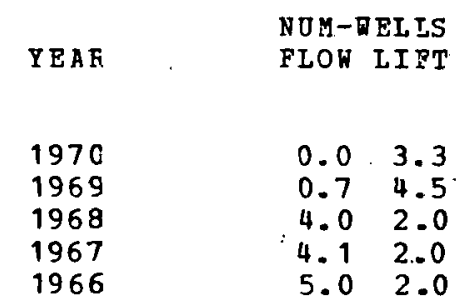

$\begin{array}{rrr}\text { OIL-PRD } & \text { E/WD } & \text { GAS-PRD } \\ & & \\ 7691 & 6.3 & 67865 \\ 24305 & 12.7 & 85956 \\ 34801 & 15.9 & 69821 \\ 35936 & 16.2 & 134745 \\ 46394 & 18.2 & 75022\end{array}$


ABANDONED ONSHORE OIL FIELDS IITH OVER 250,000 BBL. COMDLAT IVE PRODOCTION

DST DISC ABD. PLD NOM FIELD NAME

$0319601975 \quad 8871259$ BLESSING $(\mathrm{P}-12)$

\begin{tabular}{|c|c|c|c|}
\hline DEPTH & GRAV IT Y & \multicolumn{2}{|c|}{ COMUL. } \\
\hline 7,790 & 40.6 & & \\
\hline YEAR & & $\begin{array}{l}\text { NOM- } \\
\text { PLOV }\end{array}$ & $\begin{array}{l}\text { ELIS } \\
\text { LIFT }\end{array}$ \\
\hline $\begin{array}{l}1975 \\
1974 \\
1973 \\
1972 \\
1971 \\
1970 \\
1969 \\
1968 \\
1967 \\
1966\end{array}$ & & $\begin{array}{l}0.0 \\
0.0 \\
0.0 \\
0.2 \\
0.0 \\
0.3 \\
3.2 \\
5.0 \\
4.9 \\
5.1\end{array}$ & $\begin{array}{l}0.44 \\
1.0 \\
1.2 \\
3.0 \\
5.7 \\
7.7 \\
4.7 \\
3.4 \\
4.0 \\
3.5\end{array}$ \\
\hline
\end{tabular}

$\operatorname{CCONTY}$

$$
1,073,847
$$

PAT AGORD A
DST DISC ABD. PID NOA FIEID NAME

$03 \quad 1932 \quad \$ 2907001$ BDCREYE

DST DISC ABD. FID FOH PIELD NAME

$0319601973 \quad 71121052$ PHEASANT (EBERSTEIN)
DEPTH

7.750

DEP T

8,758

GRAVITY

38.8
COMOL. PROD. OF FIELD

857,457

COHOI. PROD. OP FIELD

752.487
E/RD GAS-PRD

$\begin{array}{rrr}480 & 3.2 & 2407 \\ 1904 & 5.2 & 5216 \\ 5519 & 13.0 & 4806 \\ 15847 & 13.4 & 80392 \\ 19258 & 9.3 & 116018 \\ 45647 & 15.6 & 157830 \\ 84677 & 29.0 & 184850 \\ 1.29997 & 42.3 & 224200 \\ 137021 & 42.1 & 217093 \\ 118024 & 37.7 & 161408\end{array}$

COONTY

MAT AGORDA

COONTY

IAT AGORDA

IEAR
1973
1972
1971
1970
1969
1968
1967
1966

\section{NOH-RELLS} PLOT IIFT

0.90 .8

$1.0 \quad 1.3$

$2.0 \quad 1.9$

1.12 .0

1.82 .0

2.02 .0

2.02 .0

3.01 .0

$\begin{array}{rrr}\text { OIL-PRD } & \text { E/RD } & \text { GAS-PRD } \\ & & \\ 5045 & 7.9 & 1180 \\ 18183 & 22.1 & 14548 \\ 55523 & 38.8 & 52024 \\ 14138 & 12.6 & 7599 \\ 25045 & 18.3 & 13794 \\ 37594 & 25.7 & 22541 \\ 52828 & 26.2 & 38198 \\ 78632 & 53.9 & 54332\end{array}$


ABANDONED OYSHORE OIL PIELDS MITH OVER 250,000 BBL. COMOLAT IVE PRODOCTIOA

DST DISC ABD. PLD YYTOK FI PLD NABE

031954197455468129 LOCKY (BADODGH)

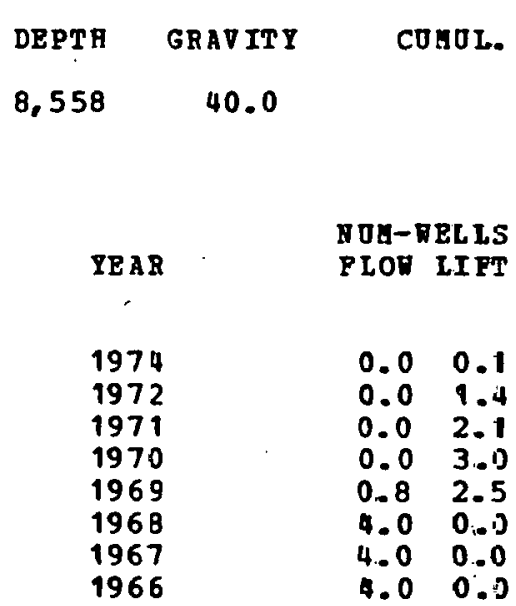

DEPTH

9.046
GRATITI

36.1
COKUL. PROD. OF PIELD

451.496
COONTY

EAT AGORDA

\footnotetext{
$03 \quad 1959 \quad 1972$

68481777 PALACIOS (BELBODRA E-4 SEG C)
}

NOB-DELIS

PLOR IIPT

Y E A R

$0.8 \quad 0.0$

$\begin{array}{ll}0.8 & 0.0 \\ 1.0 & 0.0\end{array}$

$\begin{array}{ll}1.0 & 0.0 \\ 1.0 & 0.0\end{array}$

1.00 .0

$2.70 . .2$

2.80 .2

$4.0 \quad 0.0$

1971

1969

1968
736,920

$\begin{array}{rrr}\text { OIL-PRD } & \text { E/HE } & \text { GAS-PRD } \\ & & \\ 105 & 3.5 & 0 \\ 636 & 1.2 & 14280 \\ 1615 & 2.1 & 12715 \\ 3421 & 3.1 & 16137 \\ 4202 & 3.5 & 19583 \\ 9557 & 6.5 & 28871 \\ 8516 & 5.8 & 37814 \\ 12007 & 8.2 & 100374\end{array}$

COD NTY

MAT AGORDA

$\begin{array}{rrr}\text { OIL-PRD } & \text { E/ND } & \text { GAS-PBD } \\ & & \\ 165 & 0.5 & 2928 \\ 3377 & 9.3 & 52488 \\ 4161 & 11.4 & 115645 \\ 3690 & 10.1 & 96117 \\ 5787 & 5.6 & 206807 \\ 16396 & 15.9 & 266842 \\ 24428 & 16.7 & 249164\end{array}$


ABANDONED ONSHORE OIL FIELDS RITH OVE 250,000 BBL. COUOLATIVE PRODOCTIOH

DST DISC ABD. PLD NOM PIELD NABE

$0319561970 \quad 30056333$ PAITH-HAG (PRIO 9500)

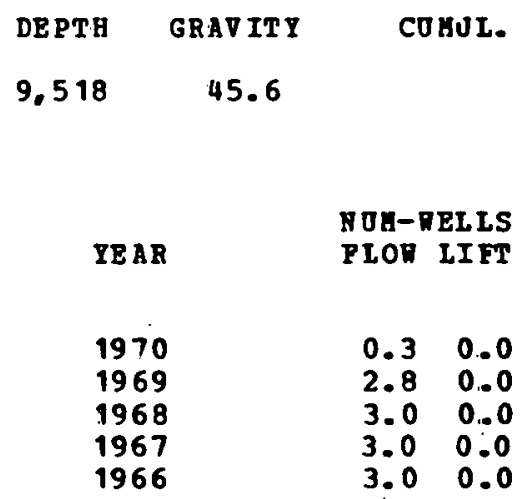

DE PTH

8, 182
GRAVITI

39.4
COUUL. PROD. OF PIELD

398,440
COO RTY

EA T AGORDA
441,897

$\begin{array}{rrr}\text { OIL-PBD } & \text { E/MC } & \text { GAS-PRD } \\ & & \\ 397 & 4.4 & 36319 \\ 9870 & 9.5 & 1075987 \\ 15438 & 14.1 & 1153224 \\ 20942 & 19.1 & 1244276 \\ 22190 & 20.3 & 1358196\end{array}$

COORTY

HAT AGOR DA

0.90 .5

\begin{tabular}{|c|c|c|}
\hline OIL-PRD & $E / R D$ & GAS-PAD \\
\hline $\begin{array}{r}12 \\
1503 \\
1365 \\
1958 \\
3013 \\
2111 \\
2713 \\
4395 \\
4241 \\
5447 \\
7939\end{array}$ & $\begin{array}{r}0.4 \\
4.1 \\
3.7 \\
5.4 \\
8.3 \\
5.8 \\
7.4 \\
12.0 \\
11.6 \\
14.9 \\
15.4\end{array}$ & $\begin{array}{r}133 \\
3603 \\
6459 \\
10549 \\
16572 \\
21236 \\
18031 \\
21260 \\
19650 \\
16578 \\
26926\end{array}$ \\
\hline
\end{tabular}


ABANDONED ONSHOPE OIL PIELDS MITH OVER 250,000 BBL. CUMULATIVE PRODUCTION

DST DISC ABD. FLD NOM FIPLD NAME

031955196961059332 MIDPIELDS TONNSTE (FRIO 8430)

\begin{abstract}
DE PTH
\end{abstract}
8,440

\section{GRAVIT Y}

49.2
COMOL. PROD. OP FIELD

371.416
CODNTY

EAT AGORDA
IEAR

1969

1968

1967

1966

\section{NOM-RELIS}

PLOT LIET

$\begin{array}{ll}0.6 & 0.4 \\ 1.0 & 0.0 \\ 1.8 & 0.2 \\ 2.3 & 0.0\end{array}$

GRAVIT T

43.5
COMUL. PROD. OP PIELD

319,087

$\begin{array}{rrr}\text { OIL-PRD } & \text { E/RI } & \text { GAS-PRD } \\ & & \\ 359 & 1: 0 & 195 \\ 3040 & 8.3 & 2963 \\ 11197 & 15: 3 & 8788 \\ 20310 & 24.7 & 17571\end{array}$

COT NTY

GAT AGORDA
7.542

YEAR

\section{NOM-RELIS}

PIOR LIFT

1972

1971

1970

1969

1968

1967

1966 $\begin{array}{ll}0.0 & 0.8 \\ 0.8 & 3.0\end{array}$

$0.8 \quad 3.0$

$1.7 \quad 4.60$

3.04 .0

$3.2 \quad 3.7$

2.94 .0
$1: 0 \quad 3.2$
OIL-PRD E/HD GAS-PRD

$\begin{array}{rrr}372 & 1.4 & 2138 \\ 5526 & 3.9 & 26383 \\ 9574 & 6.3 & 57374 \\ 13943 & 6.7 & 78367 \\ 21190 & 8.3 & 114956 \\ 22366 & 8.8 & 115961 \\ 17925 & 7.1 & 145080\end{array}$


ABANDONED ONSHORE OIL FIELDS WITH OVER 250,000 BBL. CUMULATIVE FRODUCTION

DST DISC 49D. FID NOM FIELD NAME

031960196861069830 MIDFIELDS TONNSTTE (8350)

\begin{abstract}
DEPTH
RAVITY
\end{abstract}

8,357

$43.0^{\circ}$

\section{NOM- DELIS}

YE A R

1968

1967

1966

DST DISC ARD. FLD NOM PIELD NAME

$0319631974 \quad 89926043$ TIDEHAVEN ( HEPFELFINGER)

\begin{tabular}{|c|c|c|c|}
\hline DEPTH & GR A V ITY & \multicolumn{2}{|c|}{ CUMO I. } \\
\hline 8,536 & 38.0 & & \\
\hline YEAR & & $\begin{array}{l}\text { NOH- } \\
\text { FLOR }\end{array}$ & $\begin{array}{l}\text { EL IS } \\
\text { LI FT }\end{array}$ \\
\hline$=$ & & & \\
\hline $\begin{array}{l}1974 \\
1973 \\
1972 \\
1971 \\
1970 \\
1969 \\
1968 \\
1967 \\
1966\end{array}$ & & $\begin{array}{l}0.7 \\
1.0 \\
1.0 \\
1.0 \\
1.0 \\
1.0 \\
1.0 \\
1.0 \\
2.0\end{array}$ & $\begin{array}{l}0.0 \\
0.0 \\
0.0 \\
0.3 \\
1.0 \\
1.0 \\
1.0 \\
0.3 \\
0.0\end{array}$ \\
\hline
\end{tabular}

C CDNTY

PATAGORDA

$$
\begin{array}{ll}
0.0 & 0.8 \\
0.0 & 1.0 \\
0.0 & 1.0
\end{array}
$$$$
297,624
$$ 
ABANDONED ONSHOFE OIL FIEIDS WITH OVER 250,000 BBL. CEUULATIVE PRODUCTION

DST DISC ABD. FID NOM FIELD NAỤE

031960197173740348 QUICRSAND CREER (FILCOX $\left.10,40 C^{(}\right) 10,444$
DEPTH GRAVITY

46.5
COMUI. PROD. OF PIELD

$1,690,109$
COUNTI

NERTON
DST DISC ABD. FLD NOH FIELD NABE

031951196823865001 DECRERS PRAIRIE, SOUTH

\begin{tabular}{lll} 
& \multicolumn{2}{l}{ NOM- MELIS } \\
FEAW & FIIFT \\
& & \\
1971 & 0.0 & 0.6 \\
1970 & 1.0 & 1.5 \\
1969 & 4.3 & 0.7 \\
1968 & 6.2 & 0.3 \\
1967 & 9.5 & 0.0 \\
1966 & 9.5 & 0.0
\end{tabular}

DEPTH

GRAVIT Y

5,505
OII-PRD

E/WD

1842

10930

31017

65569

202492

8.
12.
17.
28.
33.
58.

8.7
12.0

17.3

3726120

3202112

TEAR

NOM-NELIS

PLON LIFT

OIL-PRD E/HD

GA S-PRD

1968
1967

1967
1966

0.02 .0

$\begin{array}{ll}0.0 & 2.0 \\ 0.0 & 3.0\end{array}$
617
533

533
6152
COUNTY

EEWTON

690,489
G A S - P RD

$1156 \mathrm{~B}$

153486

952513

(1966


ABANDONED ONSHORE OIL PIELDS ITH OVER 250,000 BBL. CUMULATIUE PRODUCTICN
DST DISC ABD. FLD NTM FIELD NAHE
DEPTH
GRAVIT Y
COHUL. PROD. OP FIELD
COONTY
031959197173740232 QOICRSAND CREER (HILCOX, 10,260) 10,357
46.3
604,172
METTON

$\begin{array}{rrrrrr}\text { YEAR } & \begin{array}{r}\text { NOH-RELIS } \\ \text { PLON LIFT }\end{array} & \text { CIL-PRD } & \text { E/RD } & \text { GAS-PBD } \\ & & & & & \\ 1971 & 0.0 & 0.3 & 697 & 5.7 & 26196 \\ 1970 & 0.8 & 1.6 & 5999 & 6.8 & 228869 \\ 1969 & 2.9 & 1.0 & 16727 & 11.7 & 637448 \\ 1968 & 2.3 & 1.5 & 20740 & 14.8 & 839913 \\ 1967 & 4.7 & 0.8 & 31435 & 15.9 & 1191579 \\ 1966 & 4.5 & 0.0 & 73114 & 44.5 & 1204204\end{array}$

\begin{abstract}
DST DISC ABD. FLD YISM PIELD NAGE
031950 . 53075001 LEMONVILLE

DST DTSC ABD. PLD NOM FIELD NAME
\end{abstract}

031941195920219001 CONROE, DEST

\begin{tabular}{|c|c|c|c|c|}
\hline DEPTH & GRAVITY & COMU L. & PROD. OP FIBLD & COUNTY \\
\hline 7,303 & 41.4 & & 559,250 & IER TON \\
\hline DEPTH & GRAVITY & CUnOL. & PROD. OF PIBID & $\operatorname{coosT}$ \\
\hline 4,734 & 36.0 & & 463,672 & TEHTON \\
\hline
\end{tabular}


ABANDONED ONSHORE OIL FIELDS MITH OVER 250,000 BBL. CJMOLATIVE PRODUCTION

DST DISC A SD. FLD NTM PIELD NAHE
031956197339424200 AARTBORG, N. (NODOSARIA)

$\begin{array}{cc}\text { DEPTH } & \text { GRAVITY } \\ 7,545 & 41.5\end{array}$

7,545

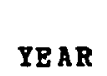

1973

1972

1971

1970

1969

1968

1967

1966

DST DISC ABD. PID NOM FIELD NAGE

DEPTH

031959197173740174 QUICKSAND CREEK (RILCOX 1J,1(10) 10,132

\section{GRAVITY}

40.1
COMOL. PROD. OF FIELD.

$$
380,065
$$

$\operatorname{conNTY}$

IEYTON

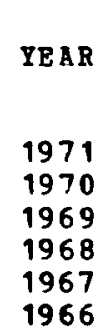

NOM-RESIS

FLOR LIET

OIL-PRD E/RE

GA S-P 3D

$\begin{array}{rr}89 & 1.5 \\ 559 & 1.5 \\ 1696 & 4.6 \\ 2723 & 7.5 \\ 7718 & 21.1 \\ 11355 & 31.1 \\ 9793 & 26.8 \\ 9141 & 25.0\end{array}$

356

$\begin{array}{ll}0.0 & 0.2 \\ 0.0 & 1.0\end{array}$

$0.0 \quad 1: 0$

$0.9 \quad 0.0$

$1.0 \quad 0.0$

$\begin{array}{ll}1.0 & 0.0 \\ 1.0 & 0.0\end{array}$

9141

COHEL. PROD. OP FIELD

$\cos \operatorname{sT} Y$

289,271

IENTON

TEAR
1971
1970
1969
1968
1967
1966

$$
\begin{array}{ll}
0.5 & 3.1 \\
1.0 & 0.0 \\
0.9 & 1.0 \\
2.0 & 1.3 \\
2.6 & 1.1 \\
3.8 & 0.3
\end{array}
$$$$
289,271
$$

IEHTO

1937
147
3734
14584
31015
29445
23812

1966

$\begin{array}{rrr}\text { OIL-PRD } & \text { E/RD } & \text { GAS-PRD } \\ & & \\ 782 & 3.7 & 38 \\ 1496 & 4.1 & 6729 \\ 3333 & 4.8 & 14175 \\ 7998 & 6.7 & 51225 \\ 14185 & 10.6 & 32193 \\ 12740 & 8.7 & 51739\end{array}$


ABANDONED ONSHORE OIL PIEIDS RITH OVER 250,000 BBL. COMOLAT IVE PRODUCTION

DST DISC ABD. FLD NTM FIELD NAME

03196219739860360 BLOE MARSH (VILCOX B-2)
DEPTH

9,878
GRAVITY

46.3
COMOL. PROD. OP PIELD

271.080

$\operatorname{codNTY}$

KERTON

\section{DST DISC ABD. FLD NTM PIELD NAME}

$03 \quad .1951$

10385001 BON REIR

DST DISC ABD. PLD NOM FIELD NAHE

$0319581976 \quad 65398664$ NETTON, S. (YEGOA 3-A)

\begin{tabular}{|c|c|c|}
\hline \multirow[b]{2}{*}{ YEAR } & \multicolumn{2}{|c|}{ NOM-WELLS } \\
\hline & FLON & LI FT \\
\hline $\begin{array}{l}1973 \\
1972 \\
1971 \\
1970 \\
1967 \\
1963 \\
1967 \\
1965\end{array}$ & $\begin{array}{l}0.9 \\
1.0 \\
1.0 \\
1.0 \\
1.0 \\
1.0 \\
1.0 \\
1.0\end{array}$ & $\begin{array}{l}0.0 \\
0.0 \\
0.0 \\
0.0 \\
0.0 \\
0.0 \\
0.0 \\
0.0\end{array}$ \\
\hline
\end{tabular}

$\begin{array}{rrr}\text { OIL-PRD } & \text { E/HE } & \text { GAS-PRD } \\ & & \\ 1818 & 5.4 & 2329 \\ 3273 & 9.0 & 8190 \\ 4132 & 11.3 & 16808 \\ 8279 & 22.7 & 24027 \\ 14185 & 38.9 & 19677 \\ 17986 & 49.3 & 22965 \\ 20100 & 55.1 & 24810 \\ 21445 & 58.8 & 19447\end{array}$

$\begin{array}{rc}\text { DEPTH } & \text { GRAVITI } \\ 11.354 & 47.5\end{array}$

COMUL. PROD. OF PIELD

261.053

$\operatorname{COONTY}$

NEWTON

$\begin{array}{ccccc}\text { DEPTH } & \text { GRAVITY } & \text { COMOI. } & \text { PROD. OF FIELD } & \text { COUNTY } \\ 7,145 & 46.3 & & 251.565 & \text { NEHTON }\end{array}$

\section{NOM-WEL LS}

FLON IIPT

\section{$0.3 \quad 0.0$}

$\begin{array}{ll}0.3 & 0.0 \\ 0.3 & 0.0\end{array}$

$\begin{array}{ll}0.3 & 0.0 \\ 0.0 & 0.5\end{array}$

$\begin{array}{ll}0.0 & 0.5 \\ 0.0 & 0.2\end{array}$

$0.0 \quad 0.2$

0.01 .3

$0.2 \quad 1.7$

$0.9 \quad 1.1$
251.565

$\begin{array}{rrr}\text { OIL-PRD } & \text { E/HD } & \text { GAS-PRD } \\ & & \\ 3009 & 33.0 & 3168 \\ 2779 & 30.5 & 3444 \\ 2380 & 13.0 & 19182 \\ 811 & 13.3 & 4123 \\ 1 & 0.0 & 1 \\ 5993 & 12.3 & 22559 \\ 9787 & 14.6 & 21512 \\ 11470 & 15.7 & 44130\end{array}$


ABANDONED ONSHOZE OII PIELDS NITH OVER 250,000 BBL. COUULATIVE PRODUCTICN

DST DISC ABD. PLD NOH FIELD NAHE

031946196672443001 PORT NECHES, NORTH
COTNTY : ORANGE

$\begin{array}{cc}\text { DEPTH } & \text { GRAVITI } \\ 8,744 & 29.0\end{array}$

IEAR

1966

8.744

29.0
NOA-RELLS
PLOR II PT

0.01 .0

1 PIEID (S)
COUOL. PROD. OP FIELD

$4,756,042$

$\operatorname{cod} \operatorname{sT}$

CRA RGE

\section{OIL-PBD E/RD}

$49 \quad 0.1$
GA S-P RD

0

$4,756,042$ CONOLATIVE PROD. FROM ABAUDONED RIELDS 
ABANDONED ONSHORE OIL FIELDS WITH OVER 250,000 BBL. COMOLATIVE PRODOCTICN

DST NISC ABD. FLD NJM TIELD NAME

031949197520510001 COPELAND CREEK

$\begin{array}{cccc}\text { DEPTH } & \text { GRAVITY } & \text { CUMUL. PROD. OF FIELD } & \text { COONTY } \\ 7,155 & 38.2 & 593,483 & \text { EOLK }\end{array}$

YEAR

NOM TRLLS

FLOR LIFT

OIL-PRD E/RD

GA S-PRD

1975

1974

1973

1972

1971

1970

1969

1963

1967

1965

$\begin{array}{ll}0.0 & 0.7 \\ 0.0 & 0.7 \\ 0.0 & 1.0 \\ 0.0 & 1.0 \\ 0.0 & 1.0 \\ 0.0 & 1.0 \\ 0.0 & 1.0 \\ 0.0 & 2.0 \\ 0.0 & 2.0 \\ 2.0 & 0.0\end{array}$

1000

4. 1

$\begin{array}{ll}634 & 2.6 \\ 933 & 2.6\end{array}$

$\begin{array}{ll}933 & 2.6 \\ 976 & 2.7\end{array}$

$668 \quad 1.8$
$1344 \quad 3.7$

14223.9

6543900

$\begin{array}{rr}6543 & 9.0 \\ 10185 & 14.0\end{array}$

$\begin{array}{rr}10185 & 14.0 \\ 9801 & 13.4\end{array}$

840

840
59

120
120
540

1700

1800

11100

11100
13770

13770
7580

GRAVIT Y

COMO L. PROD. OF FIELD

CODNT

3.430

31.1

353.465

EOL $\mathrm{K}$

\section{NUM-RELLS}

FIOW LIFT

$\begin{array}{ll}0.0 & 0.6 \\ 0.0 & 1.0 \\ 0.0 & 2.0 \\ 0.0 & 4.0 \\ 0.0 & 4.0\end{array}$

OIL-PRD E/RD

$\begin{array}{rr}6 & 0.0 \\ 578 & 1.6 \\ 2948 & 4.0 \\ 5199 & 3.6 \\ 8450 & 5.8\end{array}$

GA S-P RD

1
3
110
149
0


ABANDONED OHSHCRE OT= FIELDS ITH OVER 250,000 BBL. COMOLATIVE PRODOCTION

DST DISC ABD. PLD NOH FIELD NAHE -

$03 \cdot 1949$
DEPTH

4,382

1 PIELD (S)
COMOL. PROD. OF PIELD

810.541

42.3

COOHTY: SAN JACIRTO

COONTY

SAN JACINTO
$---10-10-$

810,541 CUMOLATIVE PRCD. FROM AEANLONEC PIELDS 
ABANDONED ONSHORE OIL FIEIDS IITE OVER 250,000 BBL. COUOLATIVE FRODUCTION

\begin{abstract}
DST DISC ABD. PLD NOM FIBLD NALE $03 \quad 19371967 \quad 46732001$ JOES LAKE
\end{abstract}

$\begin{array}{cc}\text { DEP TH } & \text { GRAVITI } \\ 7,613 & 44.0\end{array}$

COMUL. PROD: OP FIELD

$6,475,552$
COTNTY

TYL ER

\begin{tabular}{|c|c|c|c|c|}
\hline Y EAR & $\begin{array}{l}\text { XOU-RELIS } \\
\text { PLOR LIPT }\end{array}$ & OIL-PRD & $E / M D$ & GAS-PBD \\
\hline $\begin{array}{l}1967^{\circ} \\
1966\end{array}$ & $\begin{array}{rr}0.1 & 1.5 \\
11.0 & 3.0\end{array}$ & $\begin{array}{l}1412 \\
9935\end{array}$ & $\begin{array}{l}2.4 \\
1.9\end{array}$ & $\begin{array}{r}916 \\
20697\end{array}$ \\
\hline
\end{tabular}

RST DIST ARD. PID NDY PIELD NAME

DEPTH

GRAVIT Y

COHOL. PROD. OF FIELD

COVNTY

031959197589338500 THEOVENINS CR., N. (RILCOX8400 8,494

46.2

833,565

TYI ER

YEAR

1975
1974
1973
1972
1971
1970
1969
1968
1967
1966

NOH-RELLS

FLON LIFT

$0.0 \quad 0.5$

0.0 .2 .1

0.02 .3

0.02 .0

0.02 .0

$0.0 \cdot 2.0$

0.02 .0

0.02 .0

0.13 .5

0.13 .8
OIL-PRD E/RD GA S-PRD

$\begin{array}{rrr}1164 & 6.4 & 1616 \\ 8712 & 11.5 & 10661 \\ 9563 & 11.2 & 23829 \\ 12538 & 17.2 & 54319 \\ 13307 & 18.2 & 67143 \\ 10371 & 14.2 & 50385 \\ 18545 & 25.4 & 61611 \\ 24404 & 33.4 & 47450 \\ 27116 & 20.7 & 49309 \\ 26965 & 19.3 & 41041\end{array}$


ABANDONED ONSHORE OIL FIELDS प्रITH OVER 250,000 BBL. COHOLAT IVE PRODUCTION

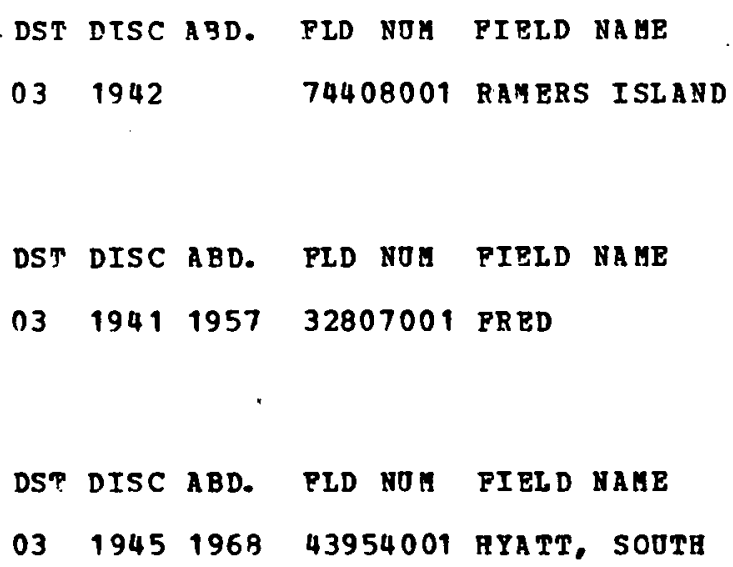

DEPTH GRAVITY

$5,406 \cdot 39.8$

CUMU1. PROD. OP PIBLD

\begin{abstract}
DEPTH GRAVITY
\end{abstract}
8,180

39.5

DEPTH GRAVITI

9,015

54.9

NOG-RELIS
TEAB . PLON IIFT

1968

1967

1966

COOHTY: TYLER
5 FIELD (S)
459,716

CCONTY

TYIER

COU HTY

339,435

TYL ER

COONTI

295,797

TYL ER

$\begin{array}{rr}\text { OIL-PRD } & \text { E/RD } \\ & \\ 1966 & 6.4 \\ 2377 & 6.5 \\ 4015 & 8.3\end{array}$

GAS-P BD

$$
\begin{array}{ll}
0.5 & 0.0 \\
1.0 & 0.0 \\
1.2 & 0.2
\end{array}
$$$$
\begin{array}{ll}
2377 & 6.5 \\
4015 & 8.3
\end{array}
$$ 
ABARDONED OHSHORE OTL FIBLDS DITH OVER 250,000 BBL. CUUULATIVE PRODUCTICA

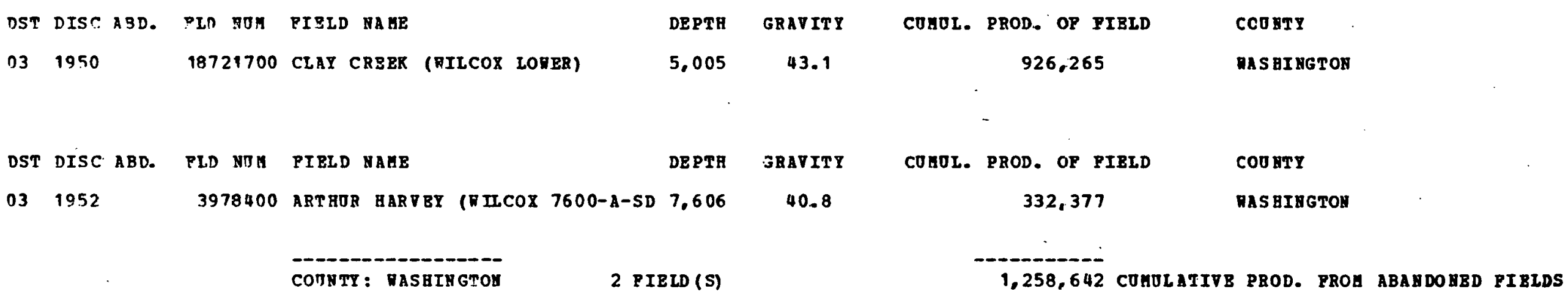


ABANDOAED OHSHORE OIL PIBLDS RITH OVER 250,000 BBL. CUMULATIVE PRODOCTION

DST DTSC ABD. PLD NOM FIELD NAME

$0319441970 \quad 27923001$ BL CAMPO

DST DISC ABD. PID WOA PIBLD HAHE

$031951 \quad 1974 \quad 72918284$ PRASIPKA (SBGHEHT MORTE)

DEPTH
6,880
YEAR
1970
1969
1968
1967
1966

GRAVITY
35.0

COUUL. PROD. OF FIELD

$1,684,855$

COONTY

DHA RTOR

$\begin{array}{lll} & \text { NOH-RELIS } \\ \text { TEAR } & \text { PLOR LIFT } \\ & & \\ 1970 & 0.0 & 0.4 \\ 1969 & 0.1 & 1.0 \\ 1968 & 0.0 & 1.3 \\ 1967 & 0.0 & 2.0 \\ 1966 & 0.7 & 1.6\end{array}$

DE PTH

GRAVITY

COHOL. PROD. OP FIELD

844,071

E/PD

5,459

25.4

yOg-nELIS

CIL-PRD E/RD

GAS-P DD

1974

1967

1966

$0.0 \cdot 0.0$

0.01 .3

0.02 .0

0.0

2.8
15.5

61 
ABANDONED ONSHORE OIL FIELDS NITH OVER 250,000 BBL. COHOLATIVE PRODOCTION

DST DISC APD. FLD MOM FIELD NAME

$03,19471972,43820001$ HTTCHINS, SOTTH

\begin{tabular}{|c|c|c|c|}
\hline DEPTH & GRAVITY & \multicolumn{2}{|c|}{ COMOL. } \\
\hline 5,876 & 30.3 & & \\
\hline & & NOM-R & EL LS \\
\hline YEER & & PLOR & IIFT \\
\hline $\begin{array}{l}1972 \\
1971 \\
1970 \\
1969 \\
1968 \\
1967 \\
1966\end{array}$ & 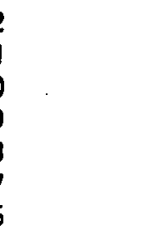 & $\begin{array}{l}0.0 \\
0.0 \\
0.1 \\
0.0 \\
0.0 \\
0.0 \\
0.0\end{array}$ & $\begin{array}{l}0.1 \\
1.0 \\
0.9 \\
1.0 \\
1.0 \\
1.0 \\
1.0\end{array}$ \\
\hline
\end{tabular}

$\begin{array}{cc}\text { DEPTH } & \text { GRAVITY } \\ 6,687 & 35.1\end{array}$

$0319631976 \quad 56688936$ MAGNET NITHERS (6600)

6,687
$Y B A R$

1976
1975
1971
1973
1972
1971
1970
1969
1968
1967
1966

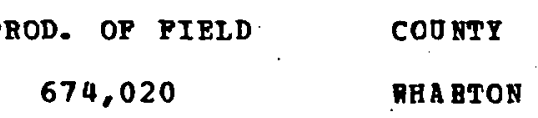


ABANDONED ONSHCRE OIL FIEIDS VITH OVER 250,000 BBL. COMULATIVE ERODUCTION

DST DISC ABD. PID NUM PIELD NAME

$031944 \quad 22688001$ DABOVAL

DST DISC ABD. FLD NDM FIELD NAME

C3 1962197556688270 MAGNET MITHERS (P-15)

DST DISC ABD. FLD NOM FIELD NAME

$0319621973 \quad 41490648$ HILLJE (PRTO 7100)

$\begin{array}{lcccc}\text { DEPTH } & \text { GRAVITY } & \text { COMUL. PROD. OP FIELD } & \text { CODNTY } \\ 7.430 & 56.7 & 457,569 & \text { FHARTON } \\ & & & & \\ \text { DEPTH } & \text { GRAVITY } & \text { COMIL. PROD. OP FIELD } & \text { CONNTY } \\ 6,551 & 34.5 & 366,995 & \text { MHARTON }\end{array}$

YEAR

NOM-WELIS

$\begin{array}{lll}1975 & 0.4 & 3.0 \\ 1974 & 0.5 & 0.2\end{array}$

$\begin{array}{lll}1974 & 0.4 & 0.0 \\ 1973 & 0.5 & 0.2\end{array}$

$\begin{array}{lll}1973 & 0.0 & 1.07 \\ 1972 & 0.0 & 1.0\end{array}$

1971

1970
1969

1968

1967

0.110 .7

0.00 .9

$\begin{array}{rr}1.8 & 0.2 \\ 2.3 & 0.2\end{array}$

$3.4 \quad 1.6$

1966

4.5 0.1

$\begin{array}{rrr}\text { OIL-PRD } & \text { E/RD } & \text { GAS-PRD } \\ & & \\ 5985 & 39.4 & 4058 \\ 19164 & 78.8 & 32428 \\ 1330 & 5.5 & 4904 \\ 1714 & 4.7 & 4063 \\ 934 & 3.4 & 7469 \\ 3948 & 11.8 & 62508 \\ 23315 & 33.3 & 355728 \\ 32104 & 36.4 & 470098 \\ 64344 & 35.3 & 654349 \\ 58132 & 34.7 & 261250\end{array}$

DEPTH GRAVITY COMOL. PROD. OP FIELD CCONTY

$7,108 \quad 38.0$

267,155

… WHARTON

Y E AR

NOM- HEL IS

FLOW L.ITT

OIL-ERD. E/WD GAS-PRD

1973

1972

1971

1970

1969

1968

1967

1966
0.10 .1

1.00 .9

1.01 .0

$\begin{array}{ll}0.2 & 1.3 \\ 0.0 & 2.0\end{array}$

0.02 .5

$\begin{array}{rr}1.2 & 2.7 \\ 4.0 & 0.0\end{array}$

6
1305
4692
5358
7728
17525
25130
36824

5400 19800 21600

21500

21600

25630

104554

14174 
ARA NDONED OKSHORE OIL PIPLDS NITH OVER 250,000 BBL. CUMULAT IVE PRODUCTION

DST DISC ABD. FID NTM FIELD NAME

031964197056693250 MAGNET NITHERS, S. (PRIO 1)

$\begin{array}{cc}\text { DEPTH } & \text { GRAVITY } \\ 5,966 & 31.4\end{array}$

COHOL. PROD. OP FIELD

257,805
COONTY

DHA BTON
YEAR

1970

1969

1968

1967

1965

COUNTY: MHARTON.

8 FIELD (5)

DISTRICT 03

117 PIEIDS

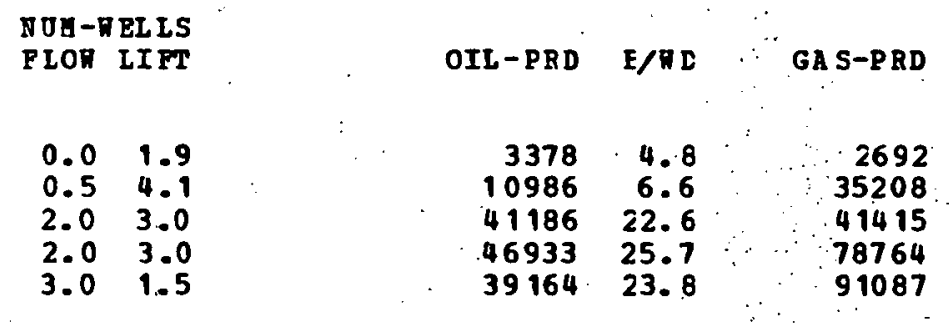

5,071,493 COMOLATIVE PROD. PROH ABAMDONED FIBLDS $144,40 \mathrm{C}, 173$ COHOLATIVE PROD. PROH ABAHCOHEC FIELDS 
APANDONPD ONSHORE OIL FIELDS NTA OVER 250,000 BBL. COMULATIVE PRODUCTICN

DST DISC ABD. PLD NOE PIELD NAME

$04 \quad 1949 \quad 197179940001$ SALT LARE

DST DISC ABD. PLD NOM FIBLD NAHE

041952.197033298777 PDLTON BEACH, EAST (E SAND)

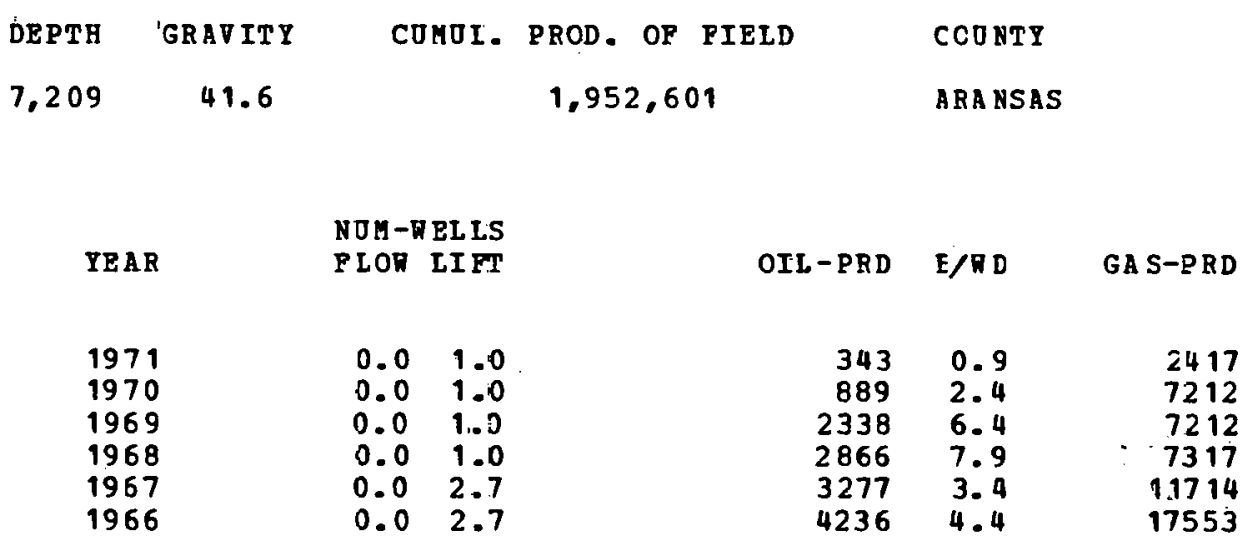

DEPTH GRAVIT I

7,715

42. 3

COMUI. PROD. OF FIELD

$\operatorname{CONNTY}$

$1,150,389$

ARANSAS

NOL-TELIS FLOR LIFT

$\begin{array}{rrr}\text { OIL-PRD } & \text { E/RD } & \text { GAS-PRD } \\ & & \\ 38 & 0.2 & 3 \\ 705 & 1.9 & 12 \\ 2086 & 5.7 & 946 \\ 2846 & 4.9 & 106 \\ 10006 & 13.7 & 0\end{array}$


ABANDONED ONSHORE OIL FIELDS MITH OVER 250,000 BBL. COHOLATIVE FRODOCTION

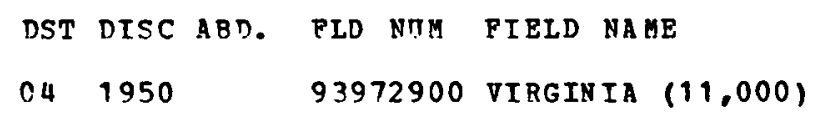

DST DISC ABD. PLD NOM PIELD NAME

041967197613466830 BORGENTINE LAKE, ST. (7700)

DST DISC ABD. PLD NOM PIELD NAME

$04 \quad 1954$

77731348 ROCK PORT, R. (-I-3 SAND)

\begin{tabular}{|c|c|c|c|}
\hline DEPTH & GRAV IT Y & \multicolumn{2}{|c|}{ COMOL. } \\
\hline 10,933 & 45.8 & & \\
\hline & & & \\
\hline DEPT日 & GR AVIT Y & co & MOL. \\
\hline 7,683 & 38.2 & & \\
\hline YEAE & & $\begin{array}{l}\text { NOH- } \\
\text { PLOR }\end{array}$ & $\begin{array}{l}\text { ELIS } \\
\text { LII FT }\end{array}$ \\
\hline $\begin{array}{l}1976 \\
1975 \\
1974 \\
1973 \\
1972 \\
1971 \\
1970 \\
1969 \\
1968 \\
1967\end{array}$ & & $\begin{array}{l}0.0 \\
0.0 \\
0.8 \\
2.7 \\
3.0 \\
4.0 \\
5.0 \\
4.9 \\
3.8 \\
0.8\end{array}$ & $\begin{array}{l}0.1 \\
1.0 \\
0.5 \\
0.0 \\
0.0 \\
0.0 \\
0.0 \\
0.0 \\
0.0 \\
0.0\end{array}$ \\
\hline
\end{tabular}

PROD. OF PIELD

CODNTY

$1,121,622$

ARA NS AS

COONTY

AR ANSAS

585,184

$\begin{array}{rrr}\text { OII-PRD } & \text { E/RD } & \text { GAS-PRD } \\ & & \\ 559 & 18.4 & 2393 \\ 8675 & 23.8 & 21157 \\ 12494 & 27.4 & 37289 \\ 15924 & 16.4 & 221189 \\ 21424 & 19.6 & 349974 \\ 42263 & 28.9 & 584911 \\ 92889 & 50.9 & 806614 \\ 158011 & 88.0 & 529717 \\ 193638 & 141.5 & 288633 \\ 39307 & 129.2 & 36773\end{array}$

DEPTH GRAVITY

COMOL. PROD. OP FIELD

COD NTY

8,821

38.5

513,469

ARANSAS 
ABANDONED ONSHOZE OIL FIZLDS WITH OVER 250,000 BBL. COMOLATIVE PRODOCTION

DST DISC ABD. FID NOM FIELD NAME

041953

33298333 FULTON BEACH, EAST $(3-2)$

DST DISC ABD. FID NOM FIELD NAME

$04 \quad 1967 \quad 1973$

13466166 SURGENTINE LAKE, SW. (A-1)

\begin{abstract}
DEPTH GRAVITY
\end{abstract}
7,135

39.8

DST DISC ABD. FLD NOM FIELD NAME

$04 \quad 1948$

51694001 LAMAR
COONTY: ARANSAS
DEPTH GRAVITY

7,673

YEAR

1973
1972
1971
1970
1969
1968
1967

40.5

CUMUL. PROD. OF PIELD

268,687

CCONTY

ARANSAS

$\begin{array}{ll}\text { NOM-WEL.IS } \\ \text { PLOW } & \text { LIFT } \\ & \\ 1.0 & 0.0 \\ 1.8 & 0.0 \\ 2.0 & 0.0 \\ 2.0 & 0.0 \\ 2.0 & 0.0 \\ 3.0 & 0.0 \\ 0.8 & 0.0\end{array}$

DEPTH

GRAVITY

CUMEI. PROD. OF FIELD

266,623

OIL - P PD

$E /$ N

383
16577

3728451.1

$37704 \quad 51.6$

4766465.3

10383594.8

$25240 \quad 92.2$

7,286

38.0

8 FIELD (S) 
ABANDONED ONSHORE OIL FIELDS ITH OVER 250,000 BBL. COHOLATIVE PRODUCTICA

DST DTSC. ABD. PLD NOM PIELD NAME

$04 \quad 1957 \quad 1975 \quad 93647804$. VIBORAS $(8,300)$

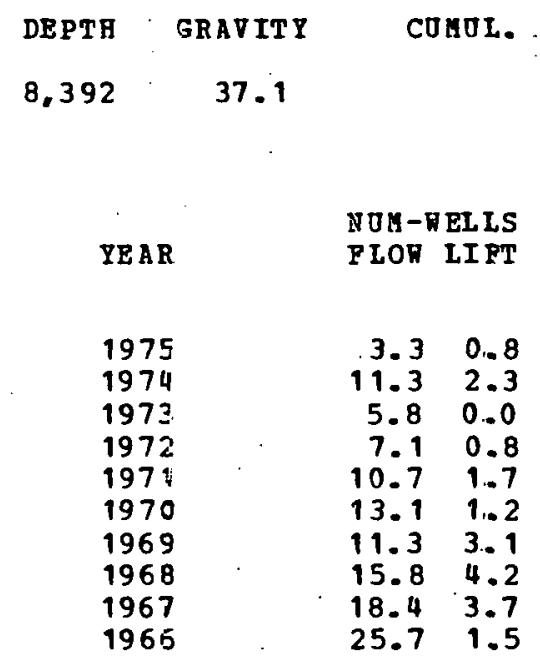

OF FIELD

$5,026,222$

COONTY

EROCKS
DST DISC ABD. FLD NOM FIELD NAME

$04 \quad 1947$
50744416 LA GLORIA (HAMMOND ZONE)

\section{DEPTH GRAVITY}

6,880
CUMOL. PROD. OP PIELD

$1,585,153$

$\begin{array}{rrr}\text { OIL-PRD } & \text { E/RD } & \text { GAS-PRD } \\ & & \\ 4598 & 0.3 & 44423 \\ 15064 & 3.0 & 1324488 \\ 50148 & 23.9 & 2931156 \\ 104261 & 36.1 & 3676019 \\ 187511 & 41.7 & 5356759 \\ 306602 & 58.9 & 5974477 \\ 410451 & 78.0 & 5918081 \\ 563241 & 77.5 & 4339747 \\ 553698 & 68.7 & 2724250 \\ 410393 & 41.4 & 2348806\end{array}$

COU NTY

EROOKS 
ABANDONED ONSHORE OIL PIELDS MITR OVER 250,000 BBL. CUMOLATIVE BRODUCTION

DST DISC ABD. FLD NOM FIELD NAGE

041945

50.744234 LA GLORIA (CHORCB ZONE)

DST DISC ABD. FID NOI FIELD NAME

041966197448631776 KELSEY, DEEP (ZOBE 22-1, N)

\section{DEPTH GRAVITY}

7. 142

39.4

DEPTH GRAVITI

6,328

34.5
COUOL. PROD. OP PIELD

850,879

CDKOL. PROD. OF PIELD

689.339
$\operatorname{COUNTY}$

EROCRS

CCOATY

EROOKS

\begin{tabular}{|c|c|c|}
\hline YRAR & NOM-K & ELAIS \\
\hline & & \\
\hline $\begin{array}{l}1974 \\
1973 \\
1972 \\
1971 \\
1970 \\
1969 \\
1968 \\
1967 \\
1966\end{array}$ & $\begin{array}{l}0.7 \\
1.3 \\
0.5 \\
1.0 \\
2.7 \\
3.5 \\
4.7 \\
4.4 \\
0.1\end{array}$ & $\begin{array}{l}0.7 \\
1.0 \\
1.1 \\
2.0 \\
1.0 \\
1.6 \\
0.8 \\
0.0\end{array}$ \\
\hline
\end{tabular}

$\begin{array}{rrr}\text { OIL-PRD } & \text { E/RD } & \text { GAS-PRD } \\ & & \\ 3354 & 6.9 & 196098 \\ 11842 & 14.4 & 503826 \\ 5273 & 10.9 & 168615 \\ 16956 & 15.5 & 403214 \\ 59259 & 44.3 & 249634 \\ 117248 & 63.2 & 485314 \\ 269026 & 134.0 & 1381232 \\ 202861 & 125.8 & 467060 \\ 2520 & 82.8 & 0\end{array}$

DST DISC ABD. FLD NDM PIELD NAME

DEPTH GRAVTTY

COGOI. PROD. OF PIELD

COONTY

5,310

41.0

656,131

EROCKS

\begin{tabular}{lll} 
& \multicolumn{2}{c}{ NUM-WELLE } \\
YEAR & PLON LIFT \\
& & \\
1975 & 0.8 & 0.0 \\
1974 & 1.0 & 0.0 \\
1973 & 1.0 & 0.0 \\
1972 & 1.7 & 0.0 \\
1971 & 2.0 & 0.0 \\
1970 & 1.9 & 0.0 \\
1969 & 2.3 & 0.0 \\
1968 & 3.0 & 0.0 \\
1967 & 5.4 & 0.0 \\
1966 & 6.9 & 0.0
\end{tabular}

GA S-PRD

$\begin{array}{rrr}1357 & 5.0 & 25316 \\ 2698 & 7.4 & 26223 \\ 6092 & 16.7 & 68171 \\ 12166 & 20.0 & 150182 \\ 18254 & 25.0 & 230300 \\ 19900 & 28.4 & 65057 \\ 25431 & 29.9 & 85031 \\ 26514 & 24.2 & 56645 \\ 47105 & 23.8 & 128215 \\ 50051 & 19.8 & 146802\end{array}$


ABANDONED ONSHORE OIL FIELDS RITH OVER 250,000 BBL. COUOLATIVE PRODOCTION

DST DISC ABD. PID NOB PIEID NAME

041946196650744039 IA GLORIA (ATLEE - B- 2ONE)

DST DISC ABD. PLD NTA FIELD NAHE

041961197693647120 VIBORAS ( P-95)

$\begin{array}{ccccc}\text { DEPTH } & \text { GRAVITY } & \text { COMOL. } & \text { PROD. OP FIELD } & \text { COONTY } \\ 6,961 & 43.4 & & 559.268 & \text { ERCGKS }\end{array}$

Y B AR

\section{N OL-RELIS}

PLOR LIPT

OII-PRD E/RD

GAS-PRD

1966

0.60 .0

1650.8

6420

$\begin{array}{ccccc}\text { DEPTH } & \text { GEAVITY } & \text { COHOL. } & \text { PROD. OF FIELD } & \text { COONTY } \\ 6,925 & 44.0 & & 386,861 & \text { EROOKS }\end{array}$

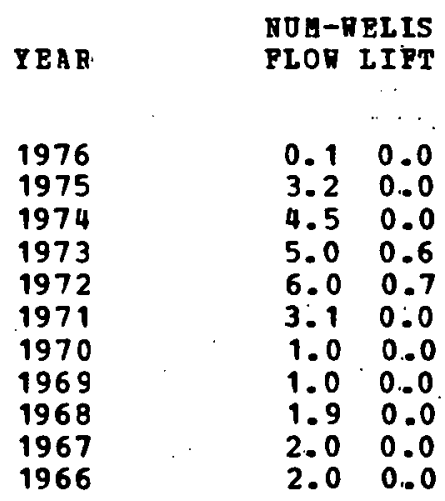

$\begin{array}{rrr}\text { OIL-PRD } & \text { E/RD } & \text { GAS-PRD } \\ & & \\ 93 & 3.1 & 15083 \\ 10009 & 8.7 & 766148 \\ 36705 & 22.3 & 2256862 \\ 60273 & 29.6 & 2174428 \\ 84818 & 34.9 & 2449834 \\ 49393 & 43.9 & 443850 \\ 4798 & 13.1 & 95277 \\ 7719 & 21.1 & 100823 \\ 14482 & 20.7 & 166523 \\ 20856 & 28.6 & 282833 \\ 14802 & 20.3 & 72373\end{array}$


ABANDONED ONSHOHE OIL FIELDS RITH OVER 250,000 B3L. CEMULAT IVE PRODUCTION

DST DISC ABD. FLD NOM FIELD NAME

041952197693647828 VIBORAS ( 8,500 SA ND)

DEPTH GRAVIT I

8,495

38.0

YEAR

1976

1975

1974

1973
1972

1971

1970

1969

1968

1967

1966

CONNTY: BROOKS
8 FIELD (S)
COMOL. PROD. OF PIELD COONTY

263,580

EROCKS

$\begin{array}{llrrr}\text { RUA-RELIS } & & & \\ \text { FIOR LIFT } & \text { OIL-PRD } & \text { E/RD } & \text { GAS-PRD } \\ & & & & \\ 0.7 & 0.0 & 3841 & 15.8 & 555330 \\ 1.0 & 0.0 & 7024 & 19.2 & 656392 \\ 1.3 & 0.0 & 6645 & 13.7 & 750886 \\ 2.7 & 0.0 & 27255 & 28.0 & 2486521 \\ 2.9 & 0.0 & 51398 & 48.3 & 4018696 \\ 2.2 & 0.0 & 44470 & 56.2 & 2600225 \\ 1.8 & 0.0 & 49782 & 74.4 & 2379213 \\ 1.1 & 0.0 & 16205 & 41.0 & 736523 \\ 1.0 & 0.0 & 15350 & 42.1 & 625583 \\ 1.0 & 0.0 & 23871 & 65.4 & 537359 \\ 2.8 & 0.0 & 5466 & 5.3 & 101825\end{array}$

$10,017,433$ COMOLATIVE PROD. PROM ABA RDONED PIELDS 
ABANDONED ONSHORE OIL FIELDS IITH OVER 250,000 BBL. COUOLATIVE PRODOCTION

DST. DISC ABD. PLD NOE FIELD NABE

$04 \quad 1945 \quad 1974 \quad 82204001$ SE JITA

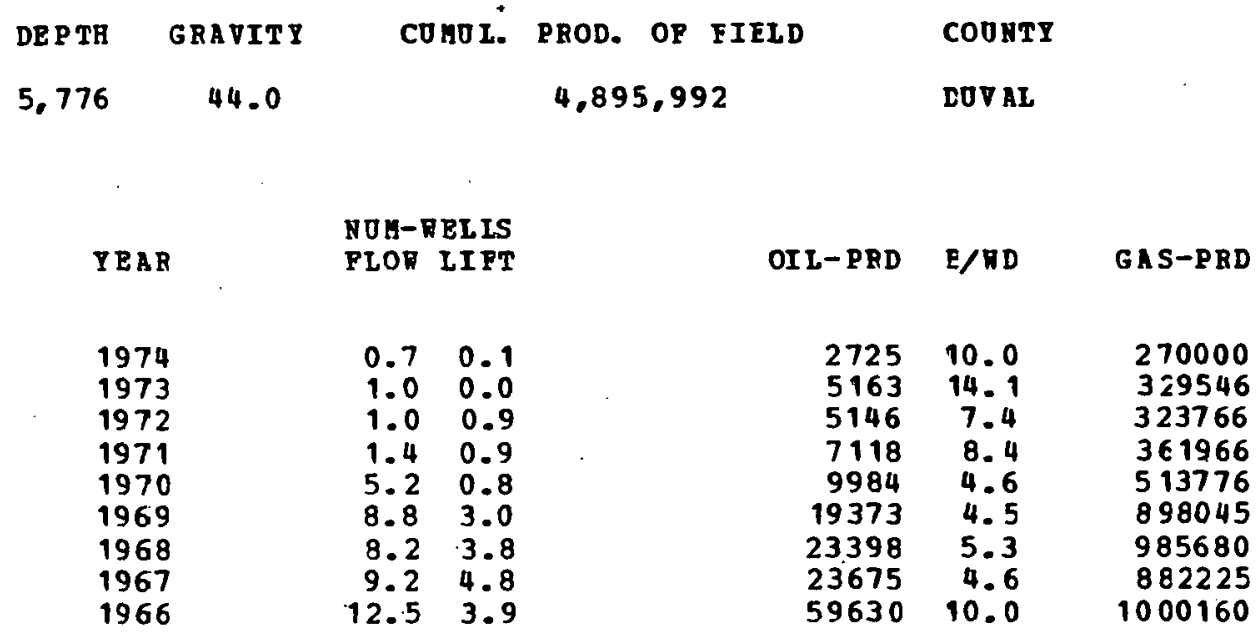

DST DISC ABD. FLD NUM FIELD NAME

$04 \quad 1939$

54756001 LONGHORN

DST DISC ABD. FLD NOM FIRLD NAME

$04 \quad 1929 \quad 1968 \quad 94756001$ SOOTHLAND
DE PTH

4,100

GRAVITY

45.7

DE PTH

3,900

GRAVITY

48.1
CUMUL. PROD. OF FIEID

$4,239,624$

COMOI. PROD. OP FIELD

$1,814,905$
COONTY

cOV AL

COONTY

cov ad

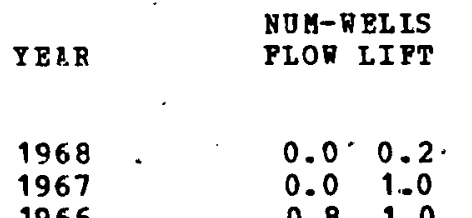

$\begin{array}{rrr}\text { OIL-PRD } & \text { E/RD } & \text { GAS-PRD } \\ & & \\ 68 & 1.1 & 1 \\ 282 & 0.8 & 43 \\ 707 & 1.1 & 257\end{array}$


ABANDONED ONSGORE OIL FIELDS RITH OVER 250,000 BEL. COUOLATIVE PRODUCTION

DST DISC ABD. FID NOA FIELD MAME

$041943 \quad 1976 \quad 77407001$ ROBINSON

DEPTE
4,649

TE AR

1976
1975
1974
1973
1972
1971
1970
1969
1968
1967
1966

DST DISC ABD. PLD NOM PIELD NAHE

$04 \quad 19571974 \quad 70842750$ PETERS, NORTH (QOEEY CITY 1ST)

\section{DEPTH \\ GRAVITY}

5,740

36.0

\section{GRAV ITY}

39.3

\section{FOM-日ELS}

FION. LIET

$\begin{array}{ll}0.0 & 0.3 \\ 0.0 & 2.5 \\ 0.0 & 3.0 \\ 0.0 & 3.0 \\ 0.0 & 3.0 \\ 0.0 & 3.0 \\ 0.0 & 3.0 \\ 0.0 & 3.0 \\ 0.0 & 3.0 \\ 0.0 & 3.0 \\ 0.0 & 3.0\end{array}$

COMUL. PROD. OF FIELD

949,206

$\begin{array}{rr}\text { OII-PRD } & \text { E/PD } \\ & \\ 129 & 1.1 \\ 1924 & 2.0 \\ 3015 & 2.8 \\ 2252 & 2.1 \\ 3683 & 3.4 \\ 3762 & 3.4 \\ 5672 & 5.2 \\ 6076 & 5.5 \\ 6265 & 5.7 \\ 6496 & 5.9 \\ 7944 & 7.3\end{array}$

CODNTY

\begin{tabular}{lll} 
& \multicolumn{2}{l}{ YOM-FELIS } \\
YEAR & PLON LIFT \\
& & \\
1974 & 0.0 & 0.2 \\
1972 & 0.0 & 3.0 \\
1971 & 0.2 & 3.2 \\
1970 & 0.0 & 4.0 \\
1969 & 0.0 & 4.0 \\
1968 & 0.0 & 4.8 \\
1967 & 0.0 & 8.0 \\
1966 & 1.0 & 7.0
\end{tabular}

$\begin{array}{rrr}\text { OIL-PRD } & \text { E/RD } & \text { GAS-PRD } \\ & & \\ 30 & 0.5 & 1 \\ 2611 & 2.4 & 568 \\ 4389 & 3.6 & 1569 \\ 5031 & 3.4 & 2726 \\ 9490 & 6.5 & 1941 \\ 11869 & 6.7 & 10081 \\ 26708 & 9.1 & 17376 \\ 78638 & 26.9 & 24539\end{array}$

CO $\nabla$ AI
GA S-P RD

$\begin{array}{rr}.1 & 25 \\ .0 & 379 \\ .8 & 1456 \\ 1 & 1293 \\ .4 & 1916 \\ .4 & 1951 \\ .2 & 3006 \\ .5 & 3530 \\ .7 & 3639 \\ .9 & 3928 \\ .3 & 0\end{array}$

COONTY

LDVAL

376 
ABANDONFD ONSHORE OII FIELDS WITH OVER 250,000 BBL. CUHULATIVE PRODUCTION

DST DISC ABD. FID IITM FIELD NAHE

$04 \quad 19431967 \quad 86463001$ STRAKE

DST DISC ABD. FID NOM FIBID NAME

$04 \quad 1948 \quad 1973 \quad 68991001$ PANTEX

$\begin{array}{ccccc}\text { DEPTH } & \text { GRAVITY } & \text { COMOL. } & \text { PROD. OP PIELD } & \text { CONNTY } \\ 3.179 & 23.6 & & 929,853 & \text { C.OVAL }\end{array}$

YEAR

NOU-RELLS

FLOT LIFT

OIL-PRD E/VE

GA S-P RD

1967

$\begin{array}{ll}0.0 & 1.0 \\ 0.0 & 2.0\end{array}$

$\begin{array}{rr}405 & 1.1 \\ 4112 & 5.6\end{array}$

8
0

1966

$\begin{array}{cc}\text { DEPTH } & \text { GRAVITY } \\ 2,593 & 23.7\end{array}$

$\begin{array}{cc}\text { DEPTH } & \text { GRAVITY } \\ 2,593 & 23.7\end{array}$

COHOI. PROD. OP PIELD

CONNTY

919,948

IOVAL

\begin{tabular}{|c|c|c|c|}
\hline YEAR & & $\begin{array}{l}\text { YOH-n } \\
\text { PLOR }\end{array}$ & $\begin{array}{l}\text { IEL IS } \\
\text { I I FT }\end{array}$ \\
\hline $\begin{array}{l}1973 \\
1972 \\
1971 \\
1969 \\
1968 \\
1967 \\
1966\end{array}$ & $\therefore$ & $\begin{array}{l}0.0 \\
0.0 \\
0.0 \\
0.0 \\
0.0 \\
0.0 \\
0.0\end{array}$ & $\begin{array}{l}3.1 \\
4.0 \\
5.0 \\
8.0 \\
7.0 \\
8.0 \\
8.0\end{array}$ \\
\hline
\end{tabular}

GAS-PRD

10
12
4
2
4
151
0 


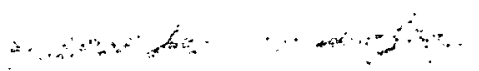

ABANDONED ONSHORE OIL FIELDS NITH OVER 250,000 BBL. COMOLATIVE PRODUCTICN

\section{DST DISC ABD. FID NDM FIELD NAME}

$04 \quad 1917$

14953001 CAM

DST DISC ABD. PLD NOM FIELD NAHE

041956197570840605 PETERS (QUEEN CITY)

$\begin{array}{ccccc}\text { DEPTH } & \text { GRAVITY } & \text { COMOI. } & \text { FROD. OP FIELD } & \text { COONTY } \\ 2.654 & 39.3 & & 392,461 & \text { IOVAL } \\ & & & & \\ \text { LEPTH } & \text { GRAVITY } & \text { COMML. } & \text { PROD. OP FIELD } & \text { CODNTY } \\ 5.708 & 39.0 & & 599,692 & \text { TOVAL }\end{array}$

\begin{tabular}{lll} 
& \multicolumn{2}{c}{ NUM-NELIS } \\
YEAR & PLON LIFT \\
& & \\
1975 & 0.0 & 1.7 \\
1974 & 0.0 & 1.6 \\
1973 & 0.0 & 1.0 \\
1972 & 0.0 & 1.0 \\
1971 & 0.0 & 1.0 \\
1970 & 0.0 & 1.7 \\
1969 & 0.0 & 2.0 \\
1968 & 0.0 & 2.0 \\
1967 & 0.0 & 3.0 \\
1966 & 0.0 & 3.0
\end{tabular}

DEPT: GRAVITY

1,849

19.4
CCONTY

464,537

COV AL

\section{O4 $19371970 \quad 74554001$ RAMCHO SOLO}

\section{NOM-HELIS}

PLOR IIPT

19.70

1969

1968

1967

1966

$$
\begin{array}{ll}
0.0 & 1.0 \\
0.0 & 1.0 \\
0.0 & 1.0 \\
0.0 & 1.0 \\
0.0 & 1.0
\end{array}
$$

$\begin{array}{rrr}\text { OIL-PRD } & \text { E/RI } & \text { GAS-PRD } \\ & & \\ 2268 & 3.7 & 571 \\ 5525 & 9.6 & 8658 \\ 4255 & 11.7 & 7313 \\ 2763 & 7.6 & 3404 \\ 2977 & 3.2 & 3499 \\ 6422 & 13.6 & 9761 \\ 7940 & 1.9 .9 & 7294 \\ 8650 & 11.8 & 6327 \\ 10760 & 9.8 & 5362 \\ 13668 & 12.5 & 6243\end{array}$

GA $-P$ RD


571
8658
7313
3404
3499
9761
7294
6327
5362
6243

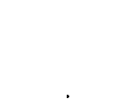

$\begin{array}{rrr}\text { OII-PRD } & \text { E/MD } & \text { GAS-PBD } \\ & & \\ 316 & 0.9 & 9 \\ 1314 & 3.6 & 12 \\ 1494 & 4.1 & 12 \\ 1451 & 4.0 & 12 \\ 1.587 & 4.3 & 0\end{array}$


ABANDONED ONSHORE OIL FIELDS WITH OVER 250,000 BBL. COMULATIVE PRODOCTION

DST TTSC ABD. FLD NOM FIELD NAME

$04 \quad 1936 \quad 1976 \quad 70840001$ PETERS

DST DLSC ABD. FLD NUM EIELD NAME

041958197154762001 LONGHORN, SOOTH

\begin{tabular}{|c|c|c|c|}
\hline DEPTH & GRAV ITY & CTMUL. PROD. OF FIELD & CODNTY \\
\hline 2,410 & 23.1 & 458,598 & LOVAL \\
\hline
\end{tabular}

YEAR

NUM-NELIS

FLOW LIFT

OIL-PRD E/WD

GA S-PRD

1976
1975

1975
1974

1974
1973

1972

1971

1970

1969

1968

1967

1966

0.0 .0 .5

0.01 .0

0.01 .0

$0.0 \quad 1.1$

0.11 .8

0.31 .8

0.02 .8

$0.3 \quad 2.7$

0.03 .0

$0.0 \quad 3.0$

$0.0 \quad 5.0$

$297 \quad 1.6$

$\begin{array}{ll}962 & 2.6 \\ 995 & 2.7\end{array}$

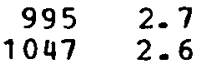

$\begin{array}{ll}1047 & 2.6 \\ 1140 & 1.6\end{array}$

$1188 \quad 1.6$

$1209 \quad 1.2$

$1557 \quad 1.4$

$1461 \quad 1.3$

$1702 \quad 1.6$

3371 1.8

.

DEPTH

GRAVITY

COMTI. PROD. OP FIELD

COUNTY

5.780

45.6

378,775

IOVAL
NOM-NELLS

FLOW LIFT

$\begin{array}{ll}0.0 & 1.0 \\ 0.0 & 1.0 \\ 0.0 & 1.0 \\ 0.0 & 1.2 \\ 0.0 & 2.0 \\ 0.0 & 2.0\end{array}$
OIL-PRD E/RD

$245 \quad 0.7$

$480 \quad 1.3$

15354.2

33327.8

$\begin{array}{ll}2960 & 4.1 \\ 2550 & 3.5\end{array}$
GAS-P BD

15
16
1473
3785
4928
10584


ABANONED ONSHORE OIL FIELDS NITH OVER 250,000 BBY.. COMOLATIVE PRODOCTION

DS" DISC ABD. PLD NOM PIELD NAME

04195019714293001 ATLEE
DEPTH
GRAVITY
5,000
47.7
COMUL. PROD. OP PIELD
377.078
$\cos N T Y$
LOVAL

\begin{tabular}{|c|c|c|c|c|c|}
\hline TEAR & $\begin{array}{l}\text { NDE- } \\
\text { FLON }\end{array}$ & $\begin{array}{l}\text { ELLS } \\
\text { LI FT }\end{array}$ & OIL - PAD & $E / D E$ & GAS-P RD \\
\hline $\begin{array}{l}1971 \\
1968 \\
1967 \\
1966\end{array}$ & $\begin{array}{l}0.7 \\
0.0 \\
0.0 \\
0.0\end{array}$ & $\begin{array}{l}0.0 \\
0.9 \\
2.3 \\
3.0\end{array}$ & $\begin{array}{l}2345 \\
1242 \\
2209 \\
7024\end{array}$ & $\begin{array}{l}9.6 \\
3.7 \\
2.6 \\
6.4\end{array}$ & $\begin{array}{r}1172 \\
870 \\
1167 \\
1530\end{array}$ \\
\hline
\end{tabular}

DST DISC ABD. PLD NTM FIELD NABE

$\begin{array}{llll}04 & 1951 & 1972 & 93813333 \text { VIGGO (IEGOA) }\end{array}$
DEPTH GRAVITY

3.700

38.0
COEUL. PROD. OP FIELD

351,698

\section{NOM-WELIS}

YEA

1972

1971
1970

1969

1968

1967

1966
PLON IIPT

$0.0 \quad 0.7$

$\begin{array}{ll}0.0 & 0.7 \\ 0.0 & 1.0 \\ 0.0 & 1.0 \\ 0.0 & 1.0 \\ 0.0 & 1.0 \\ 0.0 & 1.4 \\ 0.0 & 2.0\end{array}$

OIL-PRD

E/RD

GAS-PRD

4341.

$4293 \quad 11.8$

$\begin{array}{ll}3764 & 10.3\end{array}$

$5086 \quad 13.9$

$5906 \quad 16.2$

569411.0

$5527 \quad 7.6$ cot NTY

COV AL 
ABANDONED ONSHORE OIL FIELDS RITH OVER 250,000 BBL. COHOLATIVE PRODUCTION

\begin{abstract}
DST DISC ABD. FLD NOM DIELD NAME
$04 \quad 1949$

75040001 REALITOS, EAST
\end{abstract}

DST DISC ABD. FLD NOM EIELD NAME

$04 \quad 1950$

78193001 ROSALIA, SOUTH

DST DISC ABD. FLD NOM PIELD NAME

041958196835758666 GOOD PRIDAY (BILDA SAND)
DEPT

GRAV ITY

COMOL. PROD. OP PIELD

46.1

309,817

COONTY

4.246

DEPTH GRAVITY

$3,355 \quad 30.6$

COMOL. PROD. OP PIELD

308,263

DEPTH GRAVITY

3,562

47.9

CUMUL. PROD. OF PIELD

286,403

COUNTY

COV AL.

\begin{tabular}{|c|c|c|}
\hline & \multicolumn{2}{|c|}{ NUM-VELIS } \\
\hline YELR & & \\
\hline $\begin{array}{l}1968 \\
1967 \\
1966\end{array}$ & $\begin{array}{l}0.0 \\
0.0 \\
0.0\end{array}$ & $\begin{array}{l}0.3 \\
1.0 \\
2.0\end{array}$ \\
\hline
\end{tabular}

DE PTH

GRAVITY

DST DISC ABD. PID NOY FIELD NAME

2,937
36.2
OIL-PRD E/RD

$\begin{array}{rr}189 & 2.1 \\ 2835 & 7.8\end{array}$

$\begin{array}{rr}2835 & 7.8 \\ 7875 & 10.8\end{array}$

CUHOL. PROD. OF PIEID

270.691

COUNTY

IOV AL

\begin{tabular}{lll} 
& & \multicolumn{2}{c}{ NOM-WELIS } \\
YEA & PIOW IIPT \\
& & \\
1976 & 0.0 & 0.8 \\
1975 & 0.0 & 1.1 \\
1974 & 0.0 & 1.1 \\
1973 & 0.0 & 2.0 \\
1972 & 0.0 & 2.0 \\
1971 & 0.0 & 2.0 \\
1970 & 0.0 & 2.0 \\
1969 & 0.0 & 2.0 \\
1968 & 0.0 & 2.0 \\
1967 & 0.0 & 2.0 \\
1966 & 0.0 & 2.0
\end{tabular}

$\begin{array}{rr}\text { OIL-PRD } & \text {. E/WD } \\ & \\ 1373 & 4.5 \\ 3015 & 7.6 \\ 3093 & 7.8 \\ 3151 & 4.3 \\ 4277 & 5.9 \\ 4857 & 6.7 \\ 5362 & 7.3 \\ 5191 & 7.1 \\ 6890 & 9.4 \\ 7524 & 10.3 \\ 3516 & 4.8\end{array}$

GAS-P RD

1338 2485 
ABANDONED ONSHORE OIL PIEIDS ITH OVER 250,000 BBL. CUMOLATIVE FRODUCTION

\begin{abstract}
DST DISE ABD. FID NTE FIELD NAME
04: 1949 1969 17751001 CHILTIPIN, NORTH
\end{abstract}
DEPTH
GRAV ITY
COMOL. PROD. OP PIELD
COUNTY
4. 148
44.0
257,390
covac

\begin{tabular}{|c|c|c|c|c|}
\hline FIOR & I FT & OIL - PED & E/HD & GAS-PRD \\
\hline $\begin{array}{l}0.0 \\
0.0 \\
0.0 \\
0.0\end{array}$ & $\begin{array}{l}0.6 \\
1.0 \\
1.0 \\
1.0\end{array}$ & $\begin{array}{r}622 \\
1752 \\
1990 \\
1877\end{array}$ & $\begin{array}{l}2.9 \\
4.8 \\
5.5 \\
5.1\end{array}$ & $\begin{array}{r}90 \\
253 \\
289 \\
61\end{array}$ \\
\hline
\end{tabular}

СOUNTY: DOVAL.

19 PIELD (S)

19,745,265 CUHOLATIVE PROD. PROH ABAMDONEL PIEIDS 
ABANDONED ONSHORE OII FIELDS WITH CVER 250,000 BBL. CUMULATIVZ PRODOCTICN

DST DISC ARD. FID NOH FIELD NAME

041934197180033001 SAM FORDYCE

DST DISC ABD. FID NTM PIELD NAME

$04 \quad 1939 \quad 1968 \quad 87039001$ SULIIVAN CITY

DEPTH
2,750

YEAR
1971
1970
1969
1968
1967
$196 \epsilon$

DEPTH

3,437

GRAVITY

24.0

YEAR

1968

1967

1966

\section{GRAVIT I}

21.5
NOM-VELIS
PLOW LIFT

$0.0 \quad 0.8$

$0.0 \quad 1.0$

0.01 .3

$\begin{array}{ll}0.1 & 1.7 \\ 0.0 & 1.0\end{array}$

0.01 .0

COHOL. PROD. JF FIELD

$1,692,525$

$7,934,138$
CCONTY

FIDALGO

OIL

E/W D

GAS-PRD

$\begin{array}{lll}1416 & 4.7 & 196\end{array}$

20125.5260

20120.1260

$\begin{array}{lll}2947 & 6.1 & 241 \\ 3808 & 6.0 & 239\end{array}$

$1420 \quad 3.9 \ldots 11$

16754.6

COṆTY

EI D ALGO

$\begin{array}{rrrrrr}\text { YEAR } & \begin{array}{r}\text { NDM-REIIS } \\ \text { FLOW IIPT }\end{array} & \text { OTI-PRD } & \text { E } / \text { FD } & \text { GAS-PRD } \\ & & & & & \\ 1968 & 0.0 & 1.9 & 2235 & 3.2 & 3689 \\ 1967 & 0.0 & 2.0 & 7795 & 10.7 & 14068 \\ 1966 & 0.3 & 1.8 & 11119 & 15.2 & 22976\end{array}$


ABANDONED ONSHORE OIL FIEIDS RITH OTER 250,000 BBL. COMOLATIPE FRODUCTION

\begin{abstract}
DST DTSC ABD. FID NUM FIELD NAME
$041953196 ? 31533495$ DLORES (SULLIVAN, OPEER)
\end{abstract}

DST DISC ABD. FLD NIY FIRLD NAME

$04 \quad 1958 \quad 1971$ 93046462 SHEPHERD (MCKINSEY)

\begin{tabular}{|c|c|c|c|c|c|c|}
\hline DEPTH & GRAV IT Y & Comol. & PROD. OP & PIBLD & COUNTY & \\
\hline 4,430 & 49.1 & & 942,73 & & BI D ALGO & \\
\hline YEAR & & $\begin{array}{l}\text { NOM-QRELLS } \\
\text { PLOW LIFT }\end{array}$ & & OIL -PRD & $E / R D$ & GAS-PRD \\
\hline $\begin{array}{l}1967 \\
1966\end{array}$ & & $\begin{array}{ll}0.0 & 0.3 \\
0.0 & 1.0\end{array}$ & & $\begin{array}{r}556 \\
4791\end{array}$ & $\begin{array}{r}4.6 \\
13.1\end{array}$ & $\begin{array}{r}434 \\
3675\end{array}$ \\
\hline ETH & GRAVITY & conot. & PROD. OP & PIELD & COUNTP & \\
\hline 7.238 & $43 . .4$ & & 849,15 & & EIDALGO & \\
\hline YEAR & & NOH-WELIS & & or:_-PRD & E & GAS-P RD \\
\hline $\begin{array}{l}1971 \\
1970 \\
1969 \\
1968 \\
1967 \\
1966\end{array}$ & & $\begin{array}{rr}0.8 & 0.4 \\
1.3 & 1.0 \\
1.8 & 1.3 \\
2.8 & 1.2 \\
6.6 & 1.5 \\
10.6 & 16.8\end{array}$ & & $\begin{array}{r}96 \\
4483 \\
9909 \\
11705 \\
31850 \\
34095\end{array}$ & $\begin{array}{r}0.2 \\
5.5 \\
8.8 \\
8.0 \\
10.8 \\
3.4\end{array}$ & $\begin{array}{l}153808 \\
285237 \\
277007 \\
311364 \\
154580\end{array}$ \\
\hline
\end{tabular}


ABANDONED ONSHORE OIL PIELDS ITT OVER 250,000 BBL. COMULATIVE PRODUCTICA

DST DISC ABD. PLD NOM PIELD NAME
$04 \quad 1957197470481248$ PÉnTtAs (HARTIR SAND)

\begin{tabular}{|c|c|c|c|}
\hline P TH & GRAVITI & \multicolumn{2}{|c|}{ COHO 1.} \\
\hline 4,863 & 42.5 & & \\
\hline YREA & & $\begin{array}{l}\text { ROA-n } \\
\text { PLOR }\end{array}$ & $\begin{array}{l}\text { ELIS } \\
\text { LIFT }\end{array}$ \\
\hline $\begin{array}{l}1974 \\
1973 \\
1972 \\
1971 \\
1970 \\
1969 \\
1968 \\
1957 \\
1956\end{array}$ & & $\begin{array}{l}0.0 \\
0.0 \\
0.0 \\
0.0 \\
0.0 \\
0.0 \\
0.0 \\
2.0 \\
5.0\end{array}$ & $\begin{array}{l}0.8 \\
1.9 \\
2.0 \\
2.0 \\
2.0 \\
2.0 \\
2.0 \\
1.5 \\
0.0\end{array}$ \\
\hline
\end{tabular}

DEPTE GRAVITY

3,500

65440001 NTCHOLS

$04 \quad 1940$

DST DISC A PD. PLD HOH PIBLD MAEE

041954197125318720 DOWHA (SCROSTER)

DEPTH SRAVITY

6,955

45.0

$$
\text { OII-PRD }
$$

E/RD

GAS-P RD

$\begin{array}{lll}1290 & 4.2 & 3178\end{array}$

$2379 \cdot 3.4 \quad 8053$

$23653.2 \quad 3187$

$\begin{array}{lll}5627 & 7.7 & 11420\end{array}$

$\begin{array}{lll}3019 & 4.1 & 14445\end{array}$

$\begin{array}{lll}9868 & 13.5 & 56480\end{array}$

$1486120.4 \quad 92336$

$1934715.1 . \quad 187009$

2918316.0 . 252167

\begin{tabular}{|c|c|c|}
\hline & $\nabla D H-B$ & BI. IS \\
\hline YEAR & FLOR & LIFI \\
\hline $\begin{array}{l}1071 \\
1970 \\
1969 \\
1968 \\
1967 \\
1966\end{array}$ & $\begin{array}{l}0.0 \\
0.0 \\
0.8 \\
2.0 \\
2.0 \\
1.1\end{array}$ & $\begin{array}{l}0.0 \\
1.8 \\
0.8 \\
0.8 \\
1.8 \\
1 .\end{array}$ \\
\hline
\end{tabular}

OIL-PED E/RD GAS-PRD

$1631.3 \quad 183$

$\begin{array}{lll}6355 & 17.4 & 4571\end{array}$

$23917 \quad 39.3 \quad 14393$

$\begin{array}{lll}40171 & 41.3 & 11287\end{array}$

$39906 \quad 36.4 \quad 41809$

$24299 \quad 32.0 \cdot 44634$ 
ABANDONED ONSEORE OIL FIELDS IITH OVER 250,000 BBE. CUEOLATIVE PRODUCTION

DST DISC ABD. FLD NIM FIELD NAHE

041947

DEPTH GRAVITY

7,114

41.2 a PIEID(S)
COUOI. PROD. OF PIELD

268,506

COD ATY

HID ALGO
COUNTY: HIDALGO
13,740,562 COHOLATIVE PROD. PROH ABAHDONED PIELDS 
ARANDONED ONSHORE OIL FIEIDS AITH OVER 250,000 BBL. CUMOLATIVE PRODOCTION

\section{DST TTSC ABD. PLD NUM FIELD NAME \\ $04 \quad 1924$ \\ 40448001 HENNE-WINCH-FERRIS}

DST DTSC ARD. FLD NOM PTELD NAME

$0419421972 \quad 27260270$ EAST (SEGNENT EAST)

DE P TH
1,950
DEPTH
4,739

YEAF

1972
1971
1969
1968
1967
1966

DST DISC ABD. PILD NOM PIELD NAME

D4 $19491968 \quad 27643001$ EDIASATER

DE PTH

2,096

\begin{tabular}{|c|c|c|c|c|}
\hline DE P TH & GRAVITY & COHOL. & PROD. OF FIELD & COONTY \\
\hline 1,950 & 21.0 & & $3,215,987$ & JIB HOGG \\
\hline PT & GRA P ITY & CO MO.L. & PROD. OF FIELD & COUNTI \\
\hline 39 & 51.0 & & $1,099,218$ & JI HOG \\
\hline
\end{tabular}

$\begin{array}{lll} & \text { NOH- MELIS } \\ \text { YEAR } & \text { FLOR LIFT } \\ & & \\ 1972 & 0.3 & 0.0 \\ 1971 & 0.8 & 0.0 \\ 1969 & 0.0 & 1.0 \\ 1968 & 1.1 & 0.3 \\ 1967 & 2.0 & 0.0 \\ 1966 & 2.0 & 0.0\end{array}$

$\begin{array}{rrr}\text { OIL-PRD } & \text { E/RE } & \text { GAS-PRD } \\ & & \\ 145 & 1.2 & 1550 \\ 720 & 2.4 & 12208 \\ 353 & 1.0 & 15030 \\ 1256 & 2.4 & 25994 \\ 5032 & 6.9 & 93418 \\ 7580 & 10.4 & 214398\end{array}$

GRAVITY

CUMUL. PROD. OF PIELD

COUNTY

22.0

408,531

JIM BOGG

\begin{tabular}{|c|c|c|c|c|}
\hline & NOM-WELIS & & & \\
\hline Y EAR & FLOW LIET & CI:L-PRD & $E / D D$ & GAS-PRD \\
\hline 1968 & $0 . .2$ & 140 & 2.3 & \\
\hline
\end{tabular}


ABANDONED ONSHORE OII FIEIDS RITH OVER 250,000 BBI. COMOLATIVE PRODUCTION

DST NISC ARD. PLD NOH PIELD NAME

$041953196648631940 \mathrm{KELSEY}$, DEQEP (ZONE 23)

DST DISC ABD.: PID NJM PIEID HAHE

041940197140575750 BENSHAD (5100 SAND)

$\begin{array}{ccccc}\text { DEPTE } & \text { GRAVITY } & \text { CONUL. } & \text { PROD. OP FIELD } & \text { CCONTY } \\ 6,508 & 42.7 & 386,352 & \text { JIM HCG! }\end{array}$

YEAR

YDE- TELIS
PLOR IIET

1966

0.20 .1

OII-PRD E/RL

GA S-P RD

5456.0

4941

DEPTH GRAVITY

COHUL. PROD. OP PIELD

CCO NTY

$5.140 \quad 31.1$

285,541

JI H HCGG

\begin{tabular}{|c|c|c|}
\hline & \multicolumn{2}{|c|}{ NOH-KELZS } \\
\hline YEAR & FION & LIFI \\
\hline $\begin{array}{l}1971 \\
1970 \\
1969 \\
1968 \\
1967 \\
1966\end{array}$ & $\begin{array}{l}0.0 \\
0.0 \\
0.0 \\
0.0 \\
0.0 \\
0.0\end{array}$ & $\begin{array}{l}0.7 \\
1.0 \\
1.0 \\
1.0 \\
1.0\end{array}$ \\
\hline
\end{tabular}

CIL-PRD E/VI GAS-PRD

$\begin{array}{rr}55 & 0.2 \\ 3770 & 10.3 \\ 3228 & 8.8 \\ 2631 & 7.2 \\ 3039 & 8.3 \\ 3767 & 10.3\end{array}$

0
11
12
10
12
0 
A BANDONED ONSHORE OIL FIELDS WITH OVER 250,000 BBL. COMULATIVE PRODUCTION

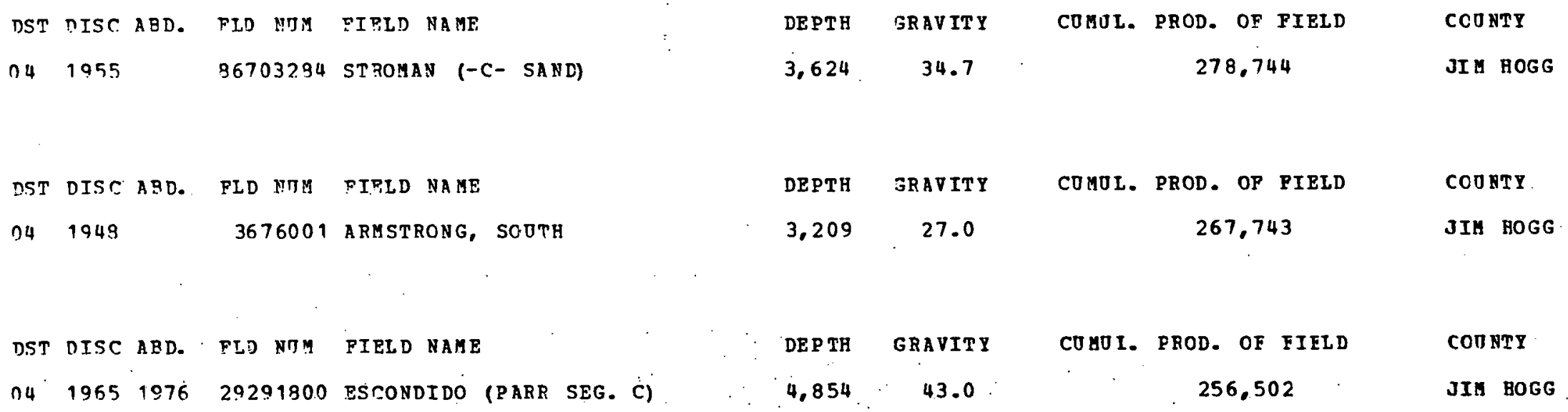
n4 $19651976 \quad 29291800$ ESCONDIDO (PARR SEG. C)
256.502
JII BOGG

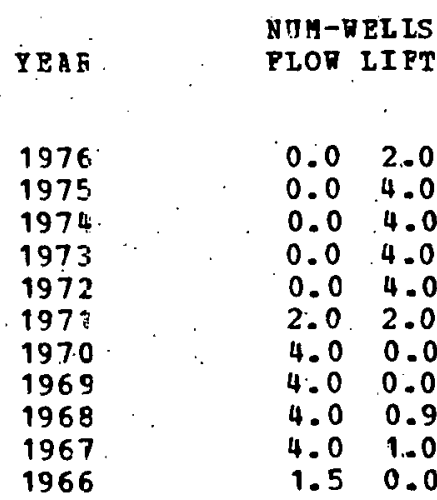

\begin{tabular}{rrr} 
OIL-PRD & E/RD & \multicolumn{1}{r}{ GAS-PBD } \\
& & \\
1772 & 2.4 & 10 \\
2716 & 1.9 & 12 \\
4484 & 3.1 & 12 \\
5208 & 3.6 & 12 \\
7976 & 5.5 & 12 \\
15236 & 10.4 & 12 \\
16944 & 11.6 & 5487 \\
16223 & 11.1 & 9036 \\
36205 & 20.2 & 23639 \\
59299 & 32.5 & 37688 \\
86380 & 157.8 & 50049
\end{tabular}


ABANDONED ONSHORE OIL FIEIDS VITH OVER 250,000 BBL. COMULATIVE PRODUCTICN

DST DISC QPD. TLD NITY FIELD NAME

ก4 1939197190493001 TOM GRAHAM

DST DTSC ABO. FID NDM FTELD NAME

$04 \quad 1943 \quad 1974 \quad 56741001$ MASNOLIA CITY, NORTHI

\begin{tabular}{|c|c|c|c|}
\hline DEPTH & GRAVITY & \multicolumn{2}{|c|}{ COMOL. } \\
\hline 5,400 & 42.6 & & \\
\hline & & \multicolumn{2}{|c|}{ NOM-NELIS } \\
\hline YEAR & & F LOW & LIFT \\
\hline $\begin{array}{l}1971 \\
1970 \\
1969 \\
1968 \\
1967 \\
1966\end{array}$ & & $\begin{array}{l}0.0 \\
0.0 \\
0.0 \\
0.0 \\
0.0 \\
0.0\end{array}$ & $\begin{array}{l}1.0 \\
1.0 \\
1.0 \\
1.0 \\
1.0 \\
1.0\end{array}$ \\
\hline
\end{tabular}

DEPTH GRAVITY

5,040

34.8

\section{COMUL. PROD. OF FIELD}

$4,385,573$
COUNT

JIM WELLS

\begin{tabular}{llr} 
& & \multicolumn{2}{c}{ NOM-WELLS } \\
YEAR & FLOW & LIFT \\
& & \\
1974 & & \\
1973 & 0.0 & 0.8 \\
1972 & 0.0 & 1.0 \\
1971 & 0.0 & 1.0 \\
1970 & 0.0 & 1.0 \\
1969 & 0.0 & 1.3 \\
1963 & 0.0 & 2.9 \\
1967 & 0.0 & 2.8 \\
1966 & 0.0 & 3.0 \\
& 1.2 & 1.8
\end{tabular}

$\begin{array}{rrr}\text { OIL-PRD } & \text { E/HD } & \text { GAS-PRD } \\ & & \\ 488 & 1.6 & 569 \\ 772 & 2.1 & 1076 \\ 1054 & 2.9 & 1430 \\ 1283 & 3.5 & 1657 \\ 1929 & 4.2 & 3499 \\ 8984 & 8.4 & 22240 \\ 10056 & 9.7 & 33891 \\ 8513 & 7.8 & 31736 \\ 11286 & 10.3 & 24019\end{array}$


ARANDONED ONSHORE OII FIELDS ITH CVER 250,000 BBL. COHOLATIVE PRODUCTICA

DST DISC ABD. FLD NOM FIBLD NAME

$041950 \quad 55078001$ LOT ELLA

DST DISC ABD. PLD NOM PIELD NAKE

$04 \quad 19371973 \quad 1435001$ ALPRED

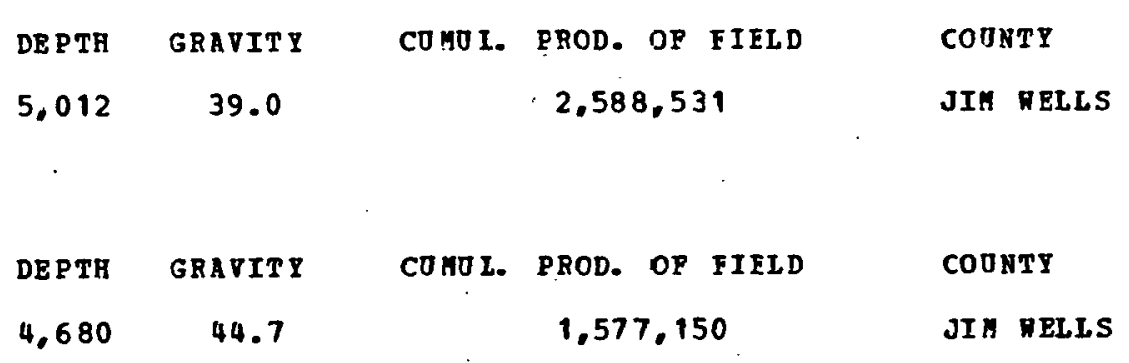

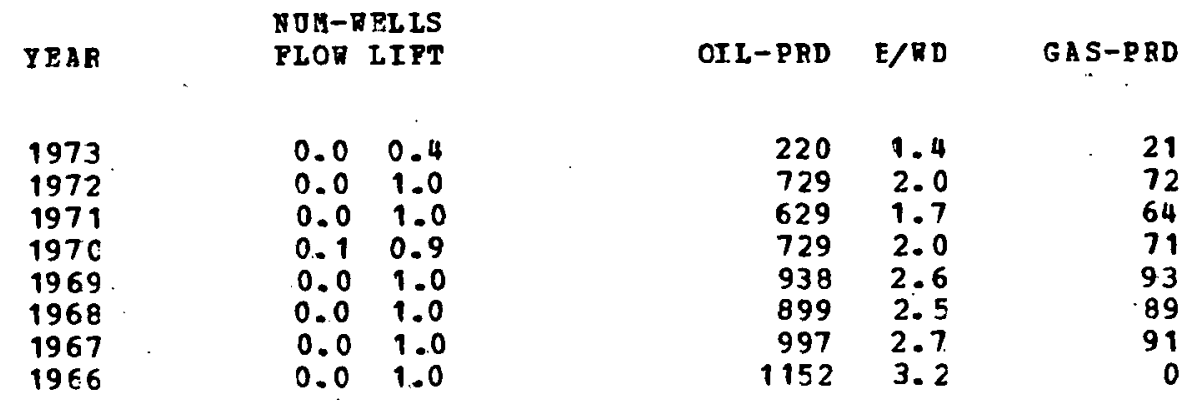

DST DISC ABD. PID NTM FIRLD NAEE

DEPTH GRATITY CUHDL. PROD. OF FIELD COUNTY

$\begin{array}{lll}4.687 & 34.9 & 935,333\end{array}$

\begin{tabular}{rrrrrr}
\multicolumn{2}{c}{ NDM-RELLS } & & \\
YEAR & FLON LIFT & OIL-PRD & E/HD & GAS-PRD \\
1974 & 0.0 & 0.3 & 228 & 2.5 & 231 \\
1959 & 0.0 & 1.3 & 1446 & 3.0 & 8600 \\
1958 & 0.0 & 1.0 & 1654 & 4.5 & 2234 \\
1967 & 0.0 & 1.7 & 1892 & 3.1 & 3740 \\
1966 & 0.0 & 2.3 & 3675 & 4.3 & 4051
\end{tabular}


ABANDONED ONSHORE OTL FIELDS WITH OVER 250,000 BBL. COMULATIVE FRODUCTICN

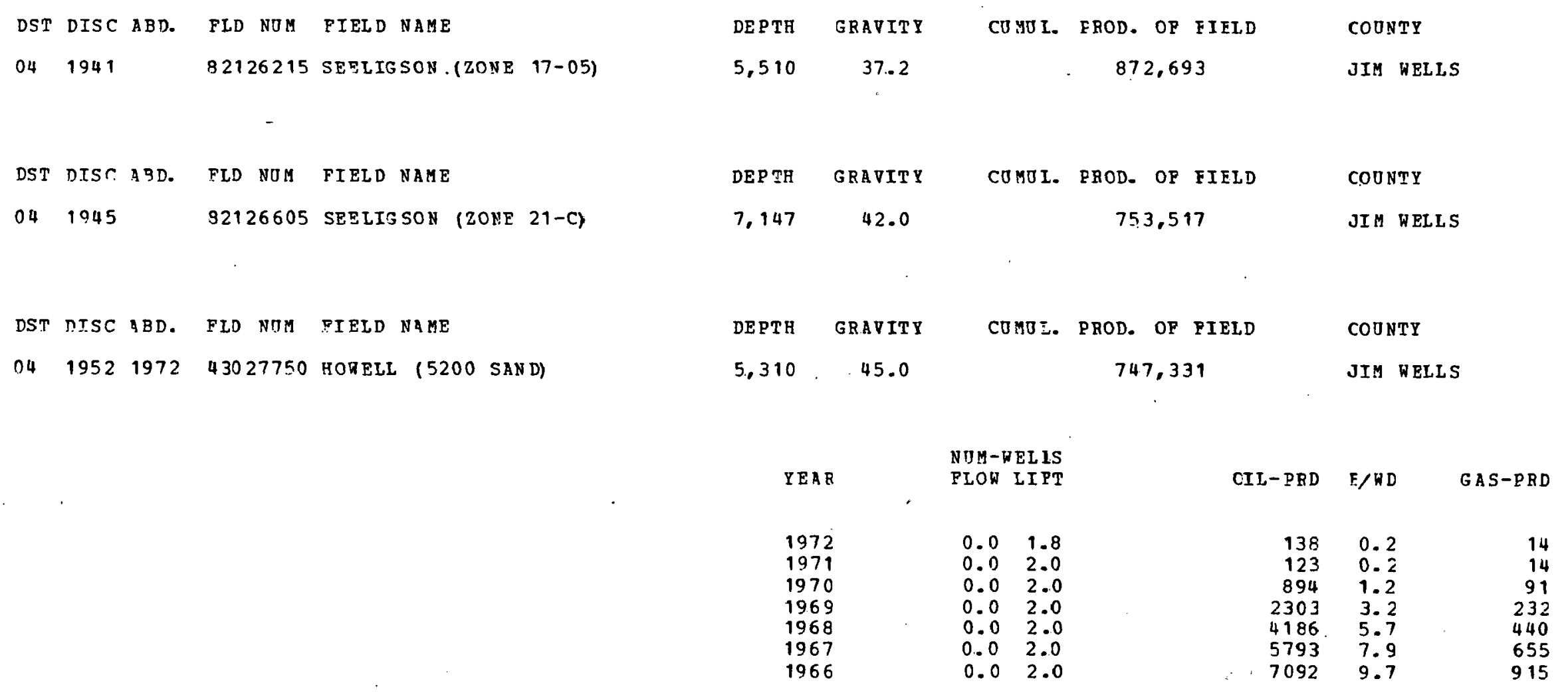


ABANDONED ONSHORE OIL FIELDS NITH OVER 250,000 BBL. COMULATIVE PRODOCTION

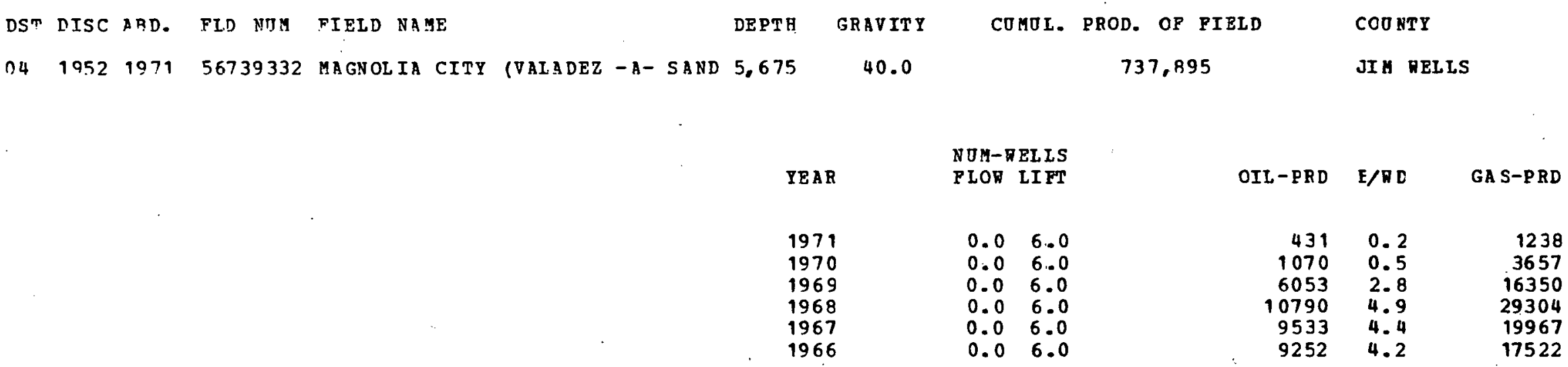

DST DTSC A.BD. FID NTY FIELD NAME

041947196938100249 HALDEMAN, SOJTH (ZONE 14-A)

\section{DE PTH \\ GRAVITY}

4. 195
43.9
COMOL. PROD. OP PIEID

657,459
COD NTY

JIM VELLS

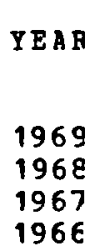

\section{NOH-RELIS}

PLOR IIPT

$\begin{array}{ll}0.0 & 0.8 \\ 0.0 & 1.8 \\ 0.0 & 2.0 \\ 0.0 & 2.0\end{array}$

OIL-PRD E/RD

GAS-PRD

$\begin{array}{rr}1671 & 6.1 \\ 2535 & 4.0\end{array}$

$8569 \quad 11.7$

10181 13.9 
49ANDONED ONSHORE OIL FEELDS WITH OVER 250,000 3BL. COBULATIVE PRODOCTICN

\section{NST DISC ABD. PLD NTM FIELD NAME}

OL $1942 \quad 73830001$ QDTNTO CRFEK

DST DIST ARD. FLD NOM FIELD NAME

OU 1952196754065333 LITTLE ROSE (PONDT SAND)

DST DTSC ABD. FLD NOM EIELD NAME

041766197482126795 SERIIGSON (ZONF 22-G-4)

$\begin{array}{ccccc}\text { DEPTH } & \text { GRAVITY } & \text { COMEI. FROD. OF FIELD } & \text { COUNTY } \\ 4,860 & 29.0 & & 371,250 & \text { JIM WELLS } \\ \text { DEPTH } & \text { GRAVITY } & \text { COMEL. PROD. OF FIELD } & \text { COUNTY } \\ 5,080 & 41.8 & & 321.883 & \text { JIM RELLS }\end{array}$

Y E A B

NOM-BELLS

FLOW LIPT

OIL-PRD E/HD

GAS-PRD

1967

1966

$$
\begin{array}{ll}
0.0 & 0.1 \\
0.0 & 0.8
\end{array}
$$

$\begin{array}{ll}240 & 7.9 \\ 726 & 2.4\end{array}$

1

DEPTH · GRAVITY

COMUI. PROD. OF FIELD

COONTY

7.721

32.0

278,476

JIM NELLS

\section{NOM-VELIS}

PLON LIPT

$\begin{array}{ll}0.5 & 0.0 \\ 1.0 & 0.0 \\ 1.0 & 0.0 \\ 1.0 & 0.0 \\ 1.0 & 0.0 \\ 1.5 & 0.3 \\ 3.0 & 1 . .3 \\ 4.0 & 1.0 \\ 2.7 & 0.0\end{array}$

OII - PRD

E/R D

GAS-P ED

1974

1973

1972

1971
1970

1969

1968

1967

$\begin{array}{rrr}1475 & 8.1 & 8864 \\ 4175 & 11.4 & 40350 \\ 6854 & 18.8 & 14531 \\ 6982 & 19.1 & 29647 \\ 9660 & 26.5 & 25542 \\ 15944 & 23.8 & 96722 \\ 39031 & 24.7 & 204580 \\ 107074 & 58.7 & 277601 \\ 87281 & 87.0 & 10027.2\end{array}$


ABANDONED ONSHORE OIL FIEIDS WITH OVER 250,000 9BL. ZUMULATIVE PRODUCTICN

\begin{tabular}{|c|c|c|c|c|c|c|c|c|c|}
\hline DST & DISC & $A B D$. & FED NTM & FIELD NAME & DE PTH & GRAVIT Y & COMO L. & FROD. OP FIELD & COONTY \\
\hline 04 & 1951 & & 15519864 & CAPTAIN LUCEY (5600 SAND) & 5,671 & 43.3 & & 276,634 & JIM NELLS \\
\hline DST & DISC & A BD. & FLD NOM & FIRID NAME & DEPTH & GRAVITY & COMUL. & PROD. OF FIEID & COONTY \\
\hline 04 & 1948 & & 82126250 & SEELIGSON (ZONE 18-C) & 5,852 & 40.0 & & 268,903 & JIM MELLS \\
\hline DST & DISC & $\mathrm{a}$ & FID NOM & FIEID NAME & DEPTH & GRAVITY & COMOI. & PROD. OF FIELD & COONTY \\
\hline 04 & 1951 & 1974 & 72947250 & EAST (ZCNE 18-A) & 4,557 & 32.5 & & 260.031 & JIM RELLS \\
\hline
\end{tabular}

\begin{tabular}{lrrrrr}
\multicolumn{1}{c}{ NUM-TELIS } & OIL-PRD & E/RD & GAS-PRD \\
YEAF & FLOF LIFT & & & \\
1974 & 0.0 & 0.4 & 409 & 2.7 & 1122 \\
1973 & 0.0 & 0.7 & 672 & 2.8 & 1912 \\
1972 & 0.0 & 1.0 & 847 & 2.3 & 7597 \\
1971 & 0.0 & 1.0 & 737 & 2.0 & 2887 \\
$197 C$ & 0.0 & 0.9 & 805 & 2.4 & 6950 \\
1959 & 0.0 & 3.3 & 11113 & 9.4 & 61975 \\
$196 E$ & 0.0 & 4.0 & 10643 & 7.3 & 13136 \\
1967 & 0.0 & 4.0 & 16816 & 11.5 & 23402 \\
1966 & 0.0 & 4.0 & 19285 & 13.2 & 19245
\end{tabular}

COONTY : JIM NELIS 16 FIELD(S)

$20,953,300$ CUMULATIVE PROD. FROM ABANDONED FIELDS 
ABANDONED ONSHOZE OII FIELDS VITH OVER 250,000 BBL. CJMOLATIVE FRODOCTICN

DST DISC ABD. PLD NOM FIELD NAME

$04 \quad 1967 \quad 1975 \quad 81115747$ SARITA (16-H)

\begin{tabular}{|c|c|c|c|c|}
\hline DEP TH & GRAVITY & CO MO L. & PROD. OF FIELD & COONTY \\
\hline .75 & 39.0 & & 444,685 & KEN EDY \\
\hline
\end{tabular}

YEA A

NUM-NELIS

FLOW IIPT

OIL-PRD E/WD

G A S - P BD

1976

1975

1974

1973

1972

1971

1970

1969

1968

1967

$\begin{array}{ll}0.0 & 0.5 \\ 0.0 & 1.0 \\ 0.1 & 0.9 \\ 0.2 & 0.8 \\ 0.0 & 1.2 \\ 0.0 & 2.4 \\ 0.0 & 4.0 \\ 1.1 & 2.9 \\ 3.1 & 0.7 \\ 1.4 & 0.0\end{array}$

$8352 \quad 45.8$

$28266 \quad 77$.

$\begin{array}{lll}32577 & 89.3\end{array}$

$28100 \quad 77.0$

$35898 \quad 84.3$

$32138 \quad 36.4$

$24263 \quad 16.6$

5113135.0

445869106.6

58091112.3

1 FIELD (S)
444,685 CUMOLATIVE PROD. PROM ABANDONED FIELDS 
ABANDONED ONSHORE OIL FIELDS HITR OVER 250,000 BBL. COUULAT IVE PRODOCTION

DST DISC ABD.: PID NTY PIELD NAME

04. 19461972 ,82126590 SRELIGSON (ZONE 21-A)

\author{
DE P TH \\ GRAVIT Y \\ 7,030
}

24.. 2

CU HOL. PROD. OP FIELD

$1,075,883$

COUNTY

KL E EERG

$\begin{array}{lrrrrr}\text { YEAR } & \begin{array}{c}\text { NOM-RELIS } \\ \text { PLOE LIPT }\end{array} & \text { OIL-PRD } & \text { E/RD } & \text { GAS-PBD } \\ & & & & & \\ 1972 & 0.2 & 0.1 & 16 & 0.2 & 670 \\ 1971 & 1.2 & 0.5 & 9977 & 16.4 & 211695 \\ 1970 & 1.4 & 0.9 & 16537 & 19.4 & 277131 \\ 1969 & 1.0 & 1.0 & 27416 & 37.6 & 257575 \\ 1963 & 1.0 & 1.0 & 36642 & 50.2 & 255947 \\ 1967 & 2.3 & 0.8 & 40600 & 36.1 & 257534 \\ 1965 & 1.1 & 0.0 & 36974 & 14.3 & 310745\end{array}$

DST DISC ABD. FID NMY FIELD NAME

DE PTH

GRAVIT T

COMOL. PROD. OP PIELD

$\operatorname{cov}$ HT

04.196619737780568 BIG CAESAR, SE. (SEG B PPL. OP) 7,570

40.6

786,114

RLEERRG

\begin{tabular}{lll} 
& \multicolumn{3}{c}{ NOM-NELIS } \\
PEAR & PLON LITT \\
& & \\
1973 & 0.0 & 0.1 \\
1972 & 0.0 & 1.7 \\
1971 & 0.6 & 2.1 \\
$197 C$ & 1.7 & 1.3 \\
1969 & 2.4 & 0.9 \\
1968 & 4.2 & 0.8 \\
1967 & 6.7 & 0.0 \\
1966 & 4.9 & 0.0
\end{tabular}

$\begin{array}{rrr}\text { OII-PRD } & \text { E/RD } & \text { GAS-PRD } \\ & & \\ 78 & 2.6 & 56 \\ 8642 & 14.2 & 3958 \\ 23571 & 24.2 & 13933 \\ 54091 & 49.4 & 29316 \\ 63400 & 52.1 & 43618 \\ 92832 & 50.9 & 125303 \\ 309519 & 127.2 & 390205 \\ 261863 & 145.9 & 206430\end{array}$


ABANDONED ONSHORE OIL FIEIDS ITT OVER 250,000 RBL. CUROLATIVE PRODOCTION

DST DTSC ABD. FLD NTM FIELD NAME

$04 \quad 1966 \quad 1976$
DEPTH GRAVITY

6,406

46. 0

YEAR
1976
1975
1974
1973
1972
1971
1970
1969
1968
1967
1966

NOH-RELIS PLON LIPT

$\begin{array}{ll}0.0 & 0.4 \\ 0.0 & 1.0 \\ 0.0 & 1.9 \\ 0.0 & 2.0 \\ 0.0 & 2.9 \\ 0.0 & 3.0 \\ 0.0 & 3.7 \\ 0.0 & 3.8 \\ 0.3 & 4.2 \\ 2.8 & 1.5 \\ 0.1 & 0.0\end{array}$

COMOI. PROD. OF FIELD

628,668

COONTY

KL BEERG
DST DISC ABD. PLD NTM FIELD VAME

DEPTH GRAVITT
35.8
COMOL. PROD. OP FIELD

504,107
GAS-PRD

1002
8157
16342
29286
23683
35582
37492
43479
96655
66252
256

$0419651974 \quad 7780284$ BIG CAESAR, SE. (PPIBUGER, LD.) 7,515

Y E A

NOM-RELIS
PLOR IIPT

OIL-PRD E/R D

GAS-PRD

1974

1973

1972

1971

1970
1969

1968

1967

1966

$$
\begin{array}{ll}
0.0 & 0.8 \\
0.0 & 1.9 \\
0.0 & 2.0 \\
0.0 & 1.9 \\
0.0 & 2.5 \\
0.2 & 3.3 \\
1.2 & 2.5 \\
4.1 & 0.5 \\
2.5 & 0.0
\end{array}
$$

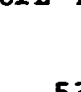


ABAYDONRD ONSHORE OII FIEIDS VITH OVER 250,000 BBL. COHOLATIVE FRODUCTION

DST DISC ABD. PLO NUM PIELD NAHE

041964197656559666 HADERO, S. (J-69-75)

\begin{tabular}{|c|c|c|c|}
\hline DEDTE & GRAV IT Y & \multicolumn{2}{|c|}{ COHOL. } \\
\hline 9,630 & 38.4 & & \\
\hline YEAR & & $\begin{array}{l}\text { NUL- } \\
\text { PLON }\end{array}$ & $\begin{array}{l}\text { EL LS } \\
\text { LI FT }\end{array}$ \\
\hline $\begin{array}{l}1976 \\
1975 \\
1974 \\
1973 \\
1972 \\
1971 \\
1970 \\
1969 \\
1968 \\
1967 \\
1966\end{array}$ & 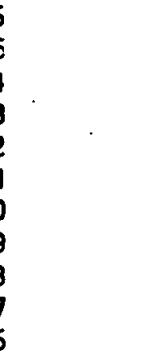 & $\begin{array}{l}0.7 \\
2.1 \\
3.0 \\
2.8 \\
2.0 \\
2.5 \\
2.1 \\
1.8 \\
2.3 \\
3.0 \\
2.3\end{array}$ & $\begin{array}{l}0.3 \\
1.0 \\
0.2 \\
0.1 \\
1.0 \\
1.0 \\
1.2 \\
2.0 \\
1.8 \\
1.0 \\
0.3\end{array}$ \\
\hline
\end{tabular}

CCUNTY

RLEER RG
DST DISC ASD. PLO NOM PIELD RAME

041964197315289728 CANELO (H-26 SD.)

$\begin{array}{ccccc}\text { DEPTH } & \text { GRAVITY } & \text { COMOI. } & \text { PROD. } 0 ? \text { FIELD } & \text { COUNTY } \\ 7.276 & 40.7 & 477,578 & \text { RLEEERG }\end{array}$

$$
539,796
$$

\begin{tabular}{rrr} 
OIL-PRD & E/HD & \multicolumn{1}{c}{ GAS-PRD } \\
& & \\
737 & 2.0 & \multicolumn{1}{c}{4349} \\
8979 & 8.0 & 56634 \\
20970 & 18.1 & 131156 \\
61983 & 59.9 & 311652 \\
33926 & 31.0 & 257.219 \\
45612 & 35.7 & 650389 \\
48879 & 41.2 & 270511 \\
40852 & 29.8 & 466994 \\
60901 & 41.7 & 1230066 \\
79835 & 54.7 & 759814 \\
60843 & 64.5 & 479284
\end{tabular}

\section{NOM- TELIS}

FIOR LIFT

OIL - PR

E/P D

GA S-P RD

$\begin{array}{ll}0.1 & 0.1 \\ 0.4 & 1.7 \\ 0.6 & 1.4 \\ 1.9 & 0.1 \\ 2.3 & 0.2 \\ 2.7 & 0.0 \\ 2.7 & 0.0 \\ 3.7 & 0.0\end{array}$

0.10 .1

1973

1972

1971

.4969

1968

1967

1966

$\begin{array}{rr}654 & 10.8 \\ 28865 & 38.0 \\ 34544 & 47.3 \\ 51018 & 69.9 \\ 56438 & 61.8 \\ 50043 & 51.4 \\ 44765 & 46.0 \\ 52967 & 39.6\end{array}$

2301 81669 51925 70840 52900 45131 45131 48001
38420 
ABANDONED ONSHORE OIL FIELDS NITA OVER 250,000 BBL. COUOLATIVE PROCUCTION

DST DISC ABD. FID NOM FTELD NAGE

$0419451974 \quad 92126650$ SEELIGSON (ZONE 21-E)

$\begin{array}{ccccc}\text { DEPTH } & \text { GRAVITY } & \text { CUMUL. } & \text { PROD. OP PIELD } & \text { CODNTY } \\ 6,906 & 32.8 & & 355,362 & \text { RLEEERG }\end{array}$

IEAR

1974

1973

1972

1971

1970

1969

1968

1967

1966

COUNTY: RLEBEFG

7 P IEUD (S)
NOM-RELLS PLOR LIFT

$\begin{array}{ll}0.8 & 0.0 \\ 0.9 & 0.0 \\ 0.8 & 0.0 \\ 1.0 & 0.0 \\ 1.0 & 0.0 \\ 1.0 & 0.0 \\ 0.8 & 0.0 \\ 1.0 & 0.0 \\ 1.0 & 0.0\end{array}$

$\begin{array}{ll}0.8 & 0.0 \\ 0.9 & 0.0\end{array}$

0.80 .0

$\begin{array}{ll}1.0 & 0.0 \\ 1.0 & 0.0\end{array}$

1.00 .0

$1.0 \quad 0.0$

355,362

RLEEERG
A S-PRD
177494
259326
474429
561441
336131
419326
43226


ABANDONED ONSHORE OIL FIEIDS HITH OVER 250,000 BBL. CUMULATIVE PRODOCTION

DST DISC ABD. FID NOM FIELD VAME

041952196937993407 HAGIST RANCH (WILCOX G)

$\begin{array}{ccccc}\text { DEPTH } & \text { GRAVITY } & \text { COMOI. } & \text { PROD. OF FIELD } & \text { COONTY } \\ 7,730 & 32.4 & 1,904,659 & \text { EOVAL }\end{array}$

YEAB

NOM-MELIS

PIOW LIPT

OIL-PRD E/RD

G.AS-P.RD

1969

1968

1967

1966.

$$
\begin{array}{ll}
0.3 & 1.8 \\
1.0 & 3.2 \\
1.0 & 7.5 \\
1.2 & 8.0
\end{array}
$$

$\begin{array}{llr}1523 & 1.9 & 39294\end{array}$

$\begin{array}{lll}4463 & 2.9 & 100245\end{array}$

$8962 \quad 2.9 \quad 131240$

$\begin{array}{rrr}8962 & 2.9 & 131240 \\ 21718 & 6.5 & 178480\end{array}$ 
ABANDONED ONSHCRE OII FIELDS HITH OVER 250,000 BBL: CUGULATIVE PRODOCTION

DST DISC ABD. FID NTM FIELD NAME

$04 \quad 1937 \quad 1969 \quad 55389001 \quad$ LOBY

DET DISC ABD. PID NOM FIRID NAME

$041936 \quad 1971 \quad 31582001$ FLODR BLUPF
DEPTH
GRAVITY
CUMUL. PRCD. OP FIELD
COONTY
4,300
45.7
$19.820,768$
NTE.CES

$\begin{array}{lrrrrr}\text { YEAR } & \begin{array}{l}\text { NOM-WELIS } \\ \text { PLOW LIPT }\end{array} & \text { OIL-PRD } & \text { E/WD } & \text { GAS-PRD } \\ & & & & & \\ 1969 & 0.2 & 1.0 & 7219 & 17.0 & 21858 \\ 1968 & 1.1 & 6.9 & 44271 & 15.3 & 160854 \\ 1967 & 0.5 & 3.2 & 57146 & 42.7 & 272183 \\ 1966 & 2.7 & 10.4 & 84273 & 17.6 & 405603\end{array}$

\begin{tabular}{|c|c|c|c|c|}
\hline DEPTH & GR A V IT Y & CU MT L. & PROD. OF FIEID & COONTY \\
\hline 6,650 & 41.5 & & 29.496 & KUECES \\
\hline
\end{tabular}

\begin{tabular}{lrrrrr}
\multicolumn{7}{c}{$\begin{array}{l}\text { NOM-WELLS } \\
\text { TEAF }\end{array}$} & FLOW LIFT & OIL-PRD & E/BD & GAS-PRD \\
& & & & & \\
1971 & 1.8 & 0.8 & 5155 & 6.7 & 148480 \\
1970 & 7.5 & 3.3 & 45684 & 11.6 & 850939 \\
1969 & 12.2 & 1.8 & 94645 & 15.3 & 1661716 \\
1968 & 18.4 & 6.7 & 168325 & 18.4 & 3190863 \\
1967 & 27.0 & 10.7 & 323530 & 23.5 & 5109867 \\
1966 & 30.2 & 12.7 & 398578 & 25.4 & 5002821
\end{tabular}


ABANDONED ONSHORE OIL FIELDS WITH OVER 250,000 BBL. CUMULATIVE PRODUCTION

DST DISC ABD. PID NOM PIELD NAME

041938

91832001 TURK EY CREER

DST DISC ABD. FLD NTM FIELD NAME

041944196911546001 BRAYTON

DST DISC ABD. FLD NTM FIELD NAME

$04 \quad 1935$

20739001 CORPOS CHRISTI

DST DISC ABD. FLD NOM FIELD NAME

$041941 \quad 1967 \quad 70904001$ PFTRONILLA
DEPTH
GRAV IT Y
COMOL. PROD. OF FIELD
COUNTY
5,800
25.0
$9,545,734$
RUECES

\begin{tabular}{|c|c|c|c|c|}
\hline DEPTH & GRAVITY & COMOL. & PROD. OF FIELD & COONTY \\
\hline 7,196 & 40.0 & & $7,681,953$ & NDECES \\
\hline
\end{tabular}

YEAR

$$
\begin{array}{ll}
\multicolumn{3}{l}{\text { NDM-KELIS }} \\
\text { FLOF } & \text { LIFT } \\
& \\
0.8 & 0.0 \\
1.0 & 0.6 \\
1.0 & 3.4 \\
1.0 & 3.5
\end{array}
$$

$$
\text { OIL-PRD E/HD }
$$

GAS-PRD

$\begin{array}{lll}1969 & 0.8 & 0.0 \\ 1968 & 1.0 & 0.6 \\ 1967 & 1.0 & 3.4 \\ 1966 & 1.0 & 3.5\end{array}$

\section{5.}

40387.0

1966

13. 1

349924

$\begin{array}{ccccc}\text { DEPTH } & \text { GRAVITY } & \text { COMUL. } & \text { PROD. OF PIEID } & \text { CCONTY } \\ 4,070 & 35.6 & 6,790,627 & \text { NOECES } \\ & & & \\ \text { DEPTH } & \text { GRAVITY } & \text { COMOI. } & \text { PROD. OF FIELD } & \text { COUNTY } \\ 7,308 & 40.0 & & 2,563,746 & \text { NUECES }\end{array}$

YEAR

NUM-NELLS

FLOW IIFT

$\begin{array}{rr}\text { CIL-PRD } & \text { E/ } \\ & \\ 58853 & 47 . \\ 72616 & 15 .\end{array}$

GAS-P RD

596522 1040935 
ABA NDONED ONSHORE OIL FIELDS WITH OVER 250,000-BBL. COMULATIVE PRODUCTICN

DST DISC ABD. PLD NOM PIBLD NAME

$0419581973 \quad 77468747$ ROBSTOWN, . EAST (5800 SAND)

DST RISC ABD. PLD NUM FIELD NABE

$041940 \quad 83230001$ SHIELD

DST DISC A9D. FLD NTM PIELD NAME

$04 \quad 19461969^{\circ} 30195001$ FARENTHOLD

$\begin{array}{ccccc}\text { DEPTH } & \text { GRAVITY } & \text { COMIL. } & \text { FROD. OF FIEID } & \text { COONTY } \\ 5,800 & 40.9 & 2,182,601 & \text { NOECES }\end{array}$

YEAR
1973
1972
1971
1970
1969
1968
1967
1966

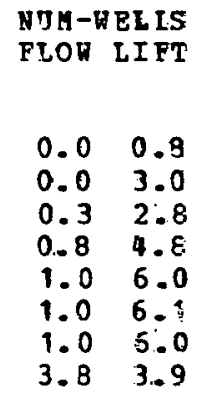

OTL-PRD E/RD

GAS-PRD

1900.7

$1048-1.0$

$\begin{array}{rr}5501 & 4.9 \\ 20005 & 10.0\end{array}$

$28598 \quad 11.2$

$28900 \quad 11.2$

$43179 \quad 16.9$

$45779 \quad 16.4$
DEPTH GRAVITY CUMJI. PROD. OP FIELD COUNTY
$6,600 \quad 44.3 \quad 1,932,069$
NOECES

DEPTH GRAVIT

COLTI. PROD. OF PIELD

CCONTY

$5,886 \quad 38.2$

$1,917,349$

KOECES

\begin{tabular}{lrrrrr}
\multicolumn{2}{c}{ NJW-WELLS } & & \\
YEAR & FLOW LIFT & OIL-PRD & E/WD & GAS-PRD \\
& & & & & \\
1969 & 0.0 & 2.0 & 653 & 0.9 & 1710 \\
1968 & 0.0 & 2.4 & 4719 & 5.3 & 10293 \\
1967 & 0.0 & 3.0 & 4380 & 4.0 & 22535 \\
1966 & 0.0 & 3.0 & 6522 & 6.0 & 18148
\end{tabular}


ABANDONED ONSBORE OIL FIELDS MITH OVER 250,000 BBL. COMOLATIOE ERODOCTION

DST DISC ARD. FLD NTM FIELD NAME

$04 \quad 19491969 \quad 5031001$ EAIL EY

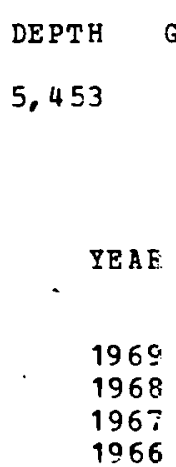

DE PTH

5,665

GRAVITY

48.2
COMUL. PROD. OP FIELD

$1,170,806$

$\operatorname{CCDNTY}$

NOECES
DST DISC ABD. FID NTM FIELD NAME

$04 \quad 19611973 \quad 3776700$ ARVOLD-DAVID $(5600)$

\begin{tabular}{lr}
\multicolumn{3}{l}{ NOM-MELLS } \\
FION \\
\\
\\
0.2 & 0.0 \\
4.2 & 1.0 \\
2.8 & 1.2 \\
6.4 & 2.5
\end{tabular}

CUMOL. PROD. OF FIELD.

COONTY

885,746

NUECES

\begin{tabular}{lrr} 
& \multicolumn{2}{c}{ NOM-FELLS } \\
YEAR & \multicolumn{2}{c}{ FIOR LIFT } \\
& & \\
1973 & 0.8 & 0.3 \\
1972 & 0.7 & 1.3 \\
1971 & 2.1 & 0.4 \\
1971 & 4.3 & 1.0 \\
1969 & 5.7 & 0.9 \\
1963 & 7.1 & 0.0 \\
1967 & 10.0 & 0.0 \\
1965 & 11.0 & 0.8
\end{tabular}

$\begin{array}{rrr}\text { ONL-PRD } & \text { E/RD } & \text { GAS-PRD } \\ & & \\ 1188 & 3.3 & 77983 \\ 5660 & 7.8 & 122114 \\ 45754 & 50.1 & 23370 \\ 50671 & 26.4 & 284200 \\ 68795 & 28.6 & 502231 \\ 94514 & 36.6 & 709614 \\ 135368 & 37.1 & 651256 \\ 107794 & 25.0 & 725460\end{array}$


A BANDONED ONSHORE OIL FIZLDS ITH OVER 250,000 BBL - COHULATIVE PRODOCTICN

DST DISC ABD. FID NOM PIELD NAME

041954197514690726 CALALLEN, NEST (7500 SAND)

\begin{tabular}{|c|c|c|c|}
\hline DE P TH & GRAVITY & \multicolumn{2}{|c|}{ CD MO L. } \\
\hline 7,491 & 36.1 & & \\
\hline Y EAR & & $\begin{array}{l}\text { NOH - } \\
\text { FLON }\end{array}$ & $\begin{array}{l}\text { EI, IS } \\
\text { LIFT }\end{array}$ \\
\hline $\begin{array}{l}1975 \\
1974 \\
1973 \\
1972 \\
1971 \\
1970 \\
1969 \\
1968 \\
1967 \\
1966\end{array}$ & & $\begin{array}{l}0.0 \\
0.0 \\
0.0 \\
0.0 \\
0.7 \\
1.1 \\
1.8 \\
1.0 \\
1.0 \\
0.2\end{array}$ & $\begin{array}{l}0.2 \\
1.0 \\
1.0 \\
1.7 \\
1.3 \\
1.7 \\
1.3 \\
2.0 \\
2.0 \\
2.1\end{array}$ \\
\hline
\end{tabular}

DST DISC ABD. PLD NOH FIBLD VAME

041940196661916448 aI THIE BOCK, N. (2 13)

$\begin{array}{cc}\text { DEPTR } & \text { GRAVIT } \\ 6.218 & 37.5\end{array}$

COMUI. PROD. OF FIELD

841.589
COONTY

NOECES

\begin{tabular}{|c|c|c|c|c|}
\hline YEAR & $\begin{array}{l}\text { NOK- } \\
\text { PLOWL LIST }\end{array}$ & OIL - PRD & E/H C & DR SA S-P \\
\hline 1966 & $0.0 \quad 0.9$ & 896 & 2.7 & 0 \\
\hline
\end{tabular}


ABANDONED ONSHORE OIL PIELDS VITH OVER 250,000 BBL. COMULATIVE PRODUCTICN

DST DISC ABD. FLD NUM FIELD NAME

$04 \quad 19391973 \quad 33912001$ GANDY

DEPTH
5,440
YEAE
1973
1972
1971
1970
1960
1968
1967
1966

DST NISC ABD. FLD NITM FIELD. NAME

$04 \quad 1930$

77047001 RIVFRSIDE

DST DISC ABD. PLD NOM FIELD NAME

$04 \quad 1956 \quad 1972 \quad 19289890 \quad \operatorname{CODY} \quad(4000 \quad \mathrm{SAND})$

5,037

\section{NOH-NELIS}

$\begin{array}{ll}0.0 & 0.5 \\ 0.0 & 1.0 \\ 0.0 & 1.0 \\ 0.0 & 1.0 \\ 0.0 & 1.0 \\ 0.0 & 1.0 \\ 0.3 & 0.8 \\ 0.7 & 0.1\end{array}$

DEPTH GRAVITY

32.2

COKUL. PROD. OF FIELD.

635.629

$\begin{array}{cc}\text { DEPTH } & \text { GRAVITY } \\ 4,035 & 24.0\end{array}$

COMUL. PROD. OP FIELD

COUNTY

597,657

$\varnothing$

NOM-WELIS

FLOW LIFT

$0.0 \quad 1.0$

0.01 .0

0.01 .0

$0.0 \quad 0.9$

0.01 .8

$\begin{array}{ll}0.0 & 2.7\end{array}$

0.0 .3 .1

COUNTY

NUECES

NOECES
OIL-PRD E/FD GAS-PRD

$803 \quad 4.4 \quad 6$

$2535 \quad 6.9 \quad 12$

$\begin{array}{lll}3382 & 9.3 & 12\end{array}$

12

$473193.0 \quad 12$

$\begin{array}{rrr}3879 & 10.6 & 12 \\ 5926 & 16.2 & 245\end{array}$

$6427 \quad 23.5 \quad 0$

\begin{tabular}{|c|c|c|}
\hline OIL-PRD & $E / W D$ & GAS-PRD \\
\hline $\begin{array}{r}1323 \\
4825 \\
3920 \\
4732 \\
4508 \\
5395 \\
15968\end{array}$ & $\begin{array}{r}3.6 \\
13.2 \\
10.7 \\
14.1 \\
7.1 \\
5.5 \\
14.2\end{array}$ & $\begin{array}{r}84 \\
748 \\
280 \\
233 \\
269 \\
1186 \\
3969\end{array}$ \\
\hline
\end{tabular}


A BAHDONED ONSHORE OIL PIELDS VITH OVER 250,000 BBL. COBOLAT IVE. RRODUCT ION

DST DISC ABD. FLD NEM FIELD NAME

$0419551965 \quad 5210759$ BALDHIN (7350)
DST DISE ABD. FLO NTM PIELD NAHE

n4. 1930

77467001 RÓBSTONN

DST DISC ABD. FLD NIM FIELD NAME

$04 \quad 19651973$
7775710 BIG CAESAR, E. (SEG C PFLETGER DP 7,384

\begin{abstract}
DEPTH
GRAVIT Y
\end{abstract}

7,385

40.0

YEAR

1966
GRAVITY

41.8
COHOL. PROD. OP FIELD

586,604

NUM-REILS

FLON LIFT

0.01 .0

DEPTH GRATITI

5,200

39.9

COHUL. PROD.: OF PIELD

551,421

COMUL. PROD. OP PIELD

545,664

C.CONTY

IOECES

E/R E

GA S-PRD

2059

5.6

5617

CCORTY

SOECES

COONTY

NOECES

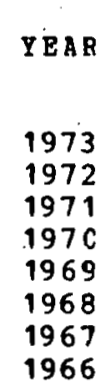

NOA-NELLS

PLON LIFT

$0.0 \quad 0.2$

0.01 .7

0.02 .7

$0.0 \quad 3.0$

0.02 .8

$\begin{array}{ll}1.7 & 0.9\end{array}$

$3.5 \quad 0.0$

$3.3 \quad 0.0$
OIL-PRD E/WD

$\begin{array}{rr}821 & 13.5 \\ 10831 & 17.8\end{array}$

$26512 \quad 27.2$

4316939.4

6579465.5

4402746.7

$89353 \cdot 69.9$

158346130.1
GAS-P $3 D$

2905
9406
37913
63093
41515
57067
125634
166006


ABANDONED ONSHORE OII FIEIDS ITA OVER 250,000 BBL C COULATIVE PRODOCTICA

DST DISC ABD. PLD NOY FIELD NAME

$0419561971^{\circ} 19299110$ CODY (CODY, LORER)

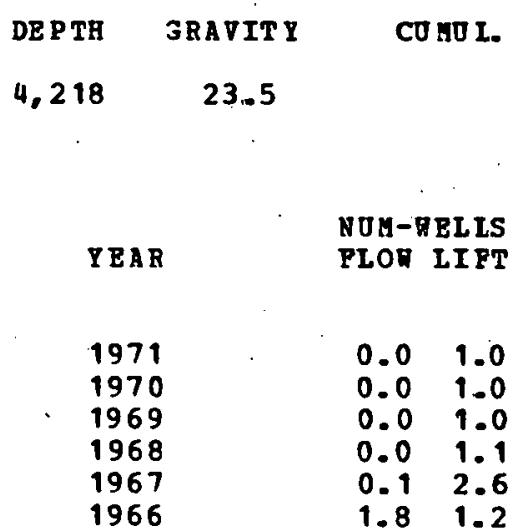

$$
483,057
$$

CONNTY

NUECES
DST DTS: A?D. FID NTM FIELD NAME

$041953 \quad 70904264$ PETRONILLA (4300 SAND)

DST DJSC AD. PLD NTI PIELD NAHE

94 1958197077458664 ROBSTONN, EAST (5600)
DEPTH

GRAVIT Y

4,337

50.3

COMTL. PROD. OP FIELD

472,042

DEPTH GRAVITY

5,650

42.0

COUOL. PROD. OF PIBID

460,452

OIL-PRD E/RD

GAS-PRD

2091 .

9443

4769

16322

16322
25804

5.7
25.9
19.9
37.4
16.8
23.6

12
12
1469
2250
9643
18596

COU BTY

KOBCBS

YEAR
1970
1969
1968
1967
1966

\section{NOM-RELIS}

PLOR LIPT

0.02 .5

0.03 .0

0.03 .3

0.04 .0

0.04 .3
OIL-PRD

E/RD

GAS-P RD

1334
45618
56050
42944
23062

1.5

41.7

46.1

29.4
14.9

23062

52
5773
7056
4971
5011

1967
1966 
ABANDONED ONSHOZE OIL FIELDS WITH OVER 250,000 BBL. COMOLATIVE FRODUCTION

DST DISC ABD. FLD NOM FIELD NAME

041956197219289165 CODY (CODY, OPPER)

DEPTH
4.205
YEAR

1972
1971
1970
1969
1968
1967
1966

DST DISC ABD. PID NOM PIELD NAHE

$0419581968 \quad 70925500$ PETRONILLA CREEK (5100 SAND)

$\begin{array}{cc}\text { DEPTH } & \text { GRAVITY } \\ 5,119 & 28.8\end{array}$

YBAR

1968

1967

1966

\section{GRAVITY}

23.5

NOM-RELLS

PLON LIPT

$0.01 . C$

$0.01 . C$

0.0 1.0

0.02 .0

0.0 3..

CUMD L. PROD. OF FIELD

445,280

$\begin{array}{rr}1540 & 4.2 \\ 3365 & 9.2 \\ 5545 & 15.2 \\ 4716 & 14.1 \\ 7584 & 13.9 \\ 11148 & 15.3 \\ 13024 & 11.9\end{array}$

GAS-PRD

67

489

420

305

1555

6788

25406 
ABA NDONED ONSHORE OIL PIEIDS MITH OVER 250,000 BBL. CUMULATIVE PRODOCTICN

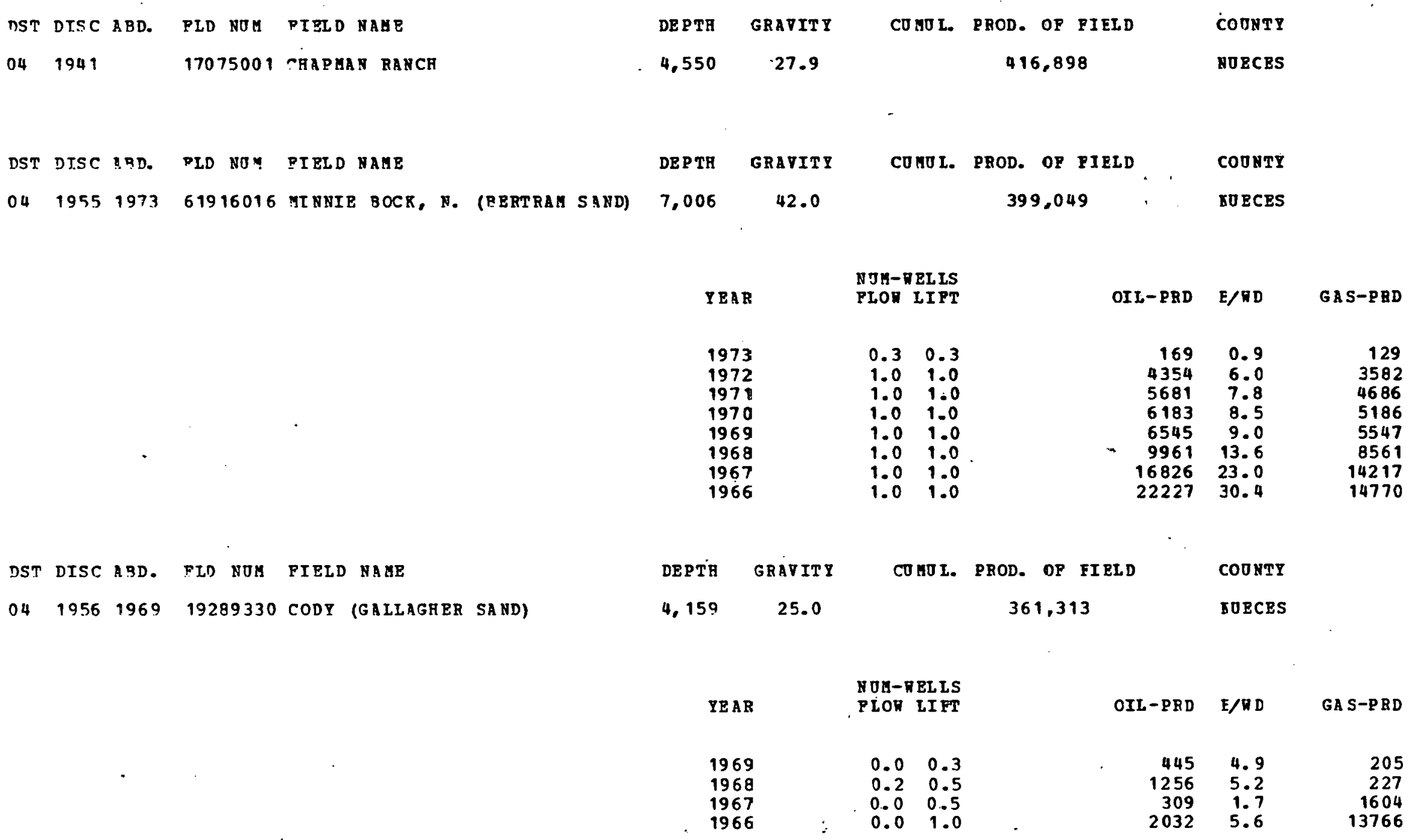


ABAEDOYED ORSHORE OII FIELDS RITH OVER 250,000 BBL. COMULATIVE PRODOCT ION

\begin{abstract}
DST DISC ABD. PID NOM PIELD MAME
\end{abstract}
04. 1964196855389403 LOBT ( 5250$)$

DST DISC ABD. PLD NOM PIELD NAME

041953197418519330 CLARA DRISCOLI, SE. (PRIO)

\begin{tabular}{|c|c|c|}
\hline DE PTH & GRAVITY & COHOL. \\
\hline 5.263 & 46.7 & \\
\hline YEAR & & $\begin{array}{l}\text { NUA-REL IS } \\
\text { FLOR LI PT }\end{array}$ \\
\hline $\begin{array}{l}1968 \\
1967 \\
1966\end{array}$ & 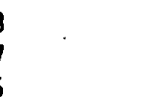 & $\begin{array}{ll}0.5 & 2.1 \\
2.8 & 2.0 \\
5.2 & 0.7\end{array}$ \\
\hline
\end{tabular}

DEPTH GRAVITY

5. 398

41.9
CUMO 1. PROD. OF FIELD

399.768

\section{IE AR}

1974

1973

1972

1971

1970
1969

1968

1967

1966

\section{RUE-VELLS}

$\begin{array}{ll}0.0 & 0.3 \\ 0.7 & 0.2 \\ 1.0 & 0.0 \\ 0.8 & 0.2 \\ 1.1 & 0.0 \\ 1.1 & 0.0 \\ 1.9 & 0.0 \\ 1.3 & 0.2 \\ 2.0 & 0.0\end{array}$

343.732

CCO RTY

IOECES 
ABANDONED ONSHORE OTL FIELDS VITH ORER 250,000 BBL. COMOLATITE PRODUCTICA

DST DISC ABD. FID NOM PIPLD NAME

041960197496509750 STRATTON, EAST (PFLUEGER, TPPER 7,092.

\begin{abstract}
DE P TH
\end{abstract}
GRAVITY

40.2

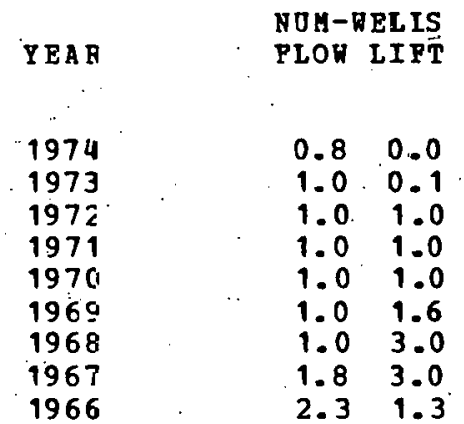

COULL. EROD. OF FIELD

309,511

COONTY

NOECES

DE PTH GRAVITI

60.3
CUMOL. PROD. OF PIELD

296,240

$\begin{array}{rrr}\text { OIL-PRD } & \text { E/WD } & \text { GAS-PRD } \\ & & \\ 806 & 2.6 & 46358 \\ 1515 & 3.8 & 124163 \\ 3115 & 4.3 & 157827 \\ 4638 & 6.4 & 172123 \\ 4958 & 6.8 & 164566 \\ 6781 & 7.2 & 191234 \\ 10982 & 7.5 & 192934 \\ 25786 & 14.6 & 173339 \\ 23951 & 18.3 & 145441\end{array}$

CCONTY

NOECES

$041961 \quad 1972 \quad 3778568$ ARNOLD-DAVID, NORTH (HIGHT SAND 6,940

\begin{tabular}{|c|c|c|}
\hline IEAS & $\begin{array}{l}\text { NUM- } \\
\text { PLON }\end{array}$ & $\begin{array}{l}\text { EL IS } \\
\text { LI FT }\end{array}$ \\
\hline $\begin{array}{l}1972 \\
1971 \\
1970 \\
1969 \\
1968 \\
1967 \\
1966\end{array}$ & $\begin{array}{l}0.0 \\
0.0 \\
0.0 \\
0.0 \\
0.0 \\
0.0 \\
0.0\end{array}$ & $\begin{array}{l}0.5 \\
1.0 \\
1.0 \\
1.0 \\
1.0 \\
1.0 \\
1.0\end{array}$ \\
\hline
\end{tabular}

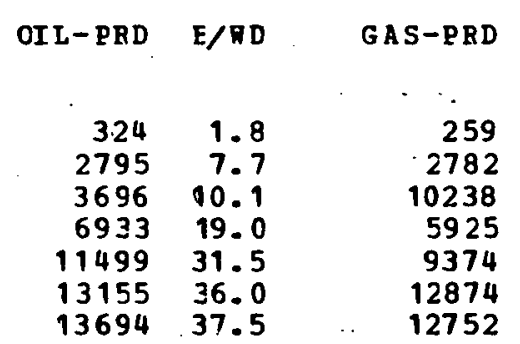


ABANDONED ONSHORE OIL FEELDS WITH OVER 250,000 BBL. CUMOLATIVE PRODOCTION

DST DEST. ABD. FLD NUM EIELD NAME

n4 196? 1973 77055333 RIVERSIDE, WRST (FRTO 6500)

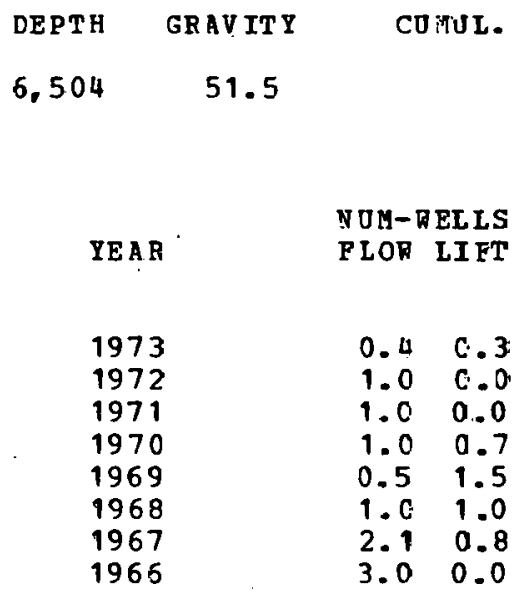

DEPTH GRAVIT Y

4,703

YEAR

1970

1969

1968

1967

1966
24.4

NJM-WEL IS

PLOW IIFT

$\begin{array}{ll}0.0 & 1.0 \\ 0.0 & 1.0 \\ 0.0 & 1.0 \\ 0.0 & 1.0 \\ 0.0 & 1.0\end{array}$
OIL - PR

$\begin{array}{rr}1560 & 6 . \\ 3620 & .9 . \\ 5604 & 15 . \\ 7401 & 12 . \\ 10085 & 13 . \\ 14645 & 20 . \\ 22312 & 21 . \\ 33025 & 30 .\end{array}$

3302530.
CCONTY

SOECES

\section{3,256}

GAS-PRD

15600

$362+6$

53053

68601

80333

99495

142560

202197

COMOL. PROD. OF FIELD. COUNTY

281.801

DOECES

OIL - PRD E/W D

GA S-P FD

$1142 \quad 3.1$

17054.7

$2489 \quad 6.8$

16024.4

$\vdots$
$\ddots$
$\vdots$
3
GA S-P FD

9
12
10
11
12


ABANDONED ONSHORE OIL PIELDS RITH OVER 250,000 BBL COBOLATIVE PRODOCTICN

DST DISC ABD. PLD NTI FIELD NAME

041957197349920500 KNOLLE, NORTE (ROERLER SAND)

DEPTH
5,320

YEA

1973
1972
1971
1970
1969
1968
1967
1966

DST DISC ABD. PID HOE FIELD NAEE

$04 \quad 1958 \quad 1971 \quad 93937250$ VI.OLET (3850)

\begin{tabular}{|c|c|c|c|}
\hline DEPTH & GRAVITY & \multicolumn{2}{|c|}{ COnOL. } \\
\hline 3,853 & 22.9 & & \\
\hline YEAR & & $\begin{array}{l}\text { YOL- } \\
\text { FLOR }\end{array}$ & $\begin{array}{l}\text { BLLS } \\
\text { LI FT }\end{array}$ \\
\hline $\begin{array}{l}1971 \\
1970 \\
1969 \\
1968 \\
1967 \\
1966\end{array}$ & & $\begin{array}{l}0.0 \\
0.0 \\
0.0 \\
0.0 \\
0.0 \\
0.0\end{array}$ & $\begin{array}{l}2.0 \\
2.0 \\
2.0 \\
2.0 \\
2.0 \\
2.0\end{array}$ \\
\hline
\end{tabular}

CODNTY

NURCES

$\begin{array}{lrrrrr}\text { TEAF } & \begin{array}{r}\text { NOH-VELIS } \\ \text { FION LIFT }\end{array} & \text { CIL-PRD } & \text { E/WD } & \text { GAS-PRD } \\ 1973 & 0.0 & 0.4 & & & \\ 1972 & 0.0 & 1.0 & 220 & 1.4 & 11 \\ 1971 & 0.0 & 1.0 & 118 & 1.7 & 144 \\ 1970 & 0.0 & 1.0 & 1289 & 3.2 & 293 \\ 1969 & 0.0 & 1.0 & 1821 & 5.0 & 324 \\ 1968 & 0.0 & 1.0 & 2173 & 6.0 & 459 \\ 1967 & 0.0 & 1.0 & 3722 & 10.2 & 1628 \\ 1966 & 0.0 & 1.0 & 3097 & 8.5 & 1350\end{array}$

28.9

.9

$\begin{array}{ll}0.0 & 1.0 \\ 0.0 & 1.0\end{array}$

281,787

COINTY

IOECES

$$
260.596
$$

$\begin{array}{rr}\text { OII-PRD } & \text { E/HD } \\ & \\ 1441 & 2.0 \\ 6082 & 8.3 \\ 7921 & 10.9 \\ 8498 & 11.6 \\ 10482 & 14.4 \\ 10999 & 15.1\end{array}$

GAS-PRD

674

3116

4960

5149

7229

6811 
ABANDONED ONSHCRE OIL PIELDS ITH OVER 250,000 BBL. COUOLATIVE PROEUCTION

DST DISC ABD. FLD NOM FIELD NAME

049958197493922684 VIOLA (6100 SAND)

DST DISC ABD. FI.D NOM FIELD NAME

O4 1953197177472498 ROBSTOWN, SODTH (560J SD)

DEPTH
6,127

YEAR

1974
1973
1972
1971
1970
1969
1968
1967
1966

DEPTH GRAVITY

5,598

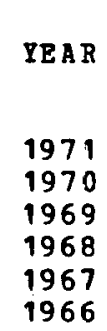

40.5
TON-HELIS

$0.0 \quad 0.5$

$0.0 \cdot 2.0$

0.02 .0

0.0 2. 0

0.02 .0

0.02 .0

0.71 .3

1.30 .8

COMGL. PROD. OF FIELD

257,840

OIL-PRD E/RE GAS-PRD

\begin{tabular}{|c|c|c|}
\hline $\begin{array}{r}208 \\
2758 \\
4230 \\
4852 \\
6146 \\
14921 \\
26036 \\
31059 \\
24346\end{array}$ & $\begin{array}{r}1.1 \\
3.8 \\
5.8 \\
6.6 \\
8.4 \\
20.4 \\
35.7 \\
40.8 \\
44.5\end{array}$ & $\begin{array}{r}3 \\
697 \\
5483 \\
5304 \\
4671 \\
17814 \\
29511 \\
43673 \\
38255\end{array}$ \\
\hline
\end{tabular}

$39346 \quad 44.5 \quad 43673$

259,249

IORCES

COONTY

IOECES

\begin{tabular}{rrr} 
& & \multicolumn{1}{c}{} \\
OIL-PRD & E/HD & GAS-PBD \\
& & \\
444 & 2.1 & 217 \\
3212 & 8.8 & 1620 \\
4977 & 13.6 & 2491 \\
5352 & 14.7 & 2671 \\
5063 & 13.9 & 2483 \\
7144 & 19.6 & 4359
\end{tabular}


ABANDONED ONSHORE OIL FIELDS NITR OVER 250,000 BBL. COMOLATIVE PRODOCTION

DST DTSC A $9 D$. FLD NOM FIELD NAME

$041951 \quad 1966 \quad 77047188$ RIVERSIDE (IOPEZ SAND)

DEPTH GRAVITY

4.870 .36 .0

YEAR

1966

COONTY : NOECES
38 PIEID (S)
COHOL. PROD. OP FIELD CCONTY

254.728

IOECES

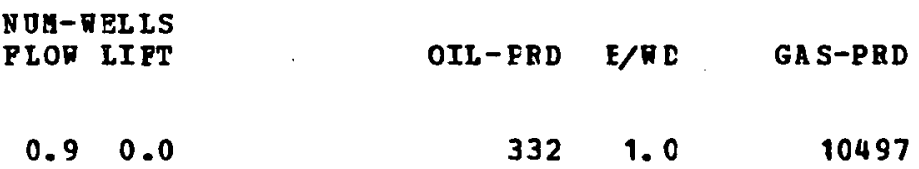

$85,179,830$ CUMOLATIVE PROD. PROM ABANDONED PIBIDS 
ABANDONED ONSHCRE OIL FIEIDS WITH OVER 250,000 BBL. COUOLATIVE PRODOCTION

DST DISC ABD. FLD NUM FIELD NAME

$04 \quad 1937 \quad 1974 \quad 61154001$ MIDWAY

DST DISC ABD. FLD NIM FIELD NAME

$04 \quad 1944 \quad 1975 \quad 83760001$ SINTON, WEST

\begin{tabular}{|c|c|c|c|c|}
\hline DE P TH & GRAVIT Y & COMOL. & PROD. OF FIELD & COONTY \\
\hline 5,229 & 28.4 & & $16,530,634$ & SAN PATRICO \\
\hline
\end{tabular}

\begin{tabular}{lll} 
& \multicolumn{2}{l}{ NUM-WELIS } \\
YEAR & FLON LIFT \\
& & \\
1974 & 0.0 & 1.2 \\
1973 & 0.0 & 2.5 \\
1972 & 0.0 & 3.3 \\
1971 & 0.0 & 3.8 \\
1970 & 0.2 & 3.1 \\
1969 & 0.0 & 3.0 \\
1968 & 0.0 & 3.0 \\
1967 & 0.3 & 3.7 \\
1966 & 1.0 & 5.0
\end{tabular}

OIL-DRD

GAS-PRD

DEPTH GRAVITY

COMUL. PROD. OF FIELD

$7,651,387$

$\begin{array}{rrr}1491 & 3.5 & 1210 \\ 7298 & 8.0 & 5203 \\ 16174 & 13.6 & 4842 \\ 19861 & 14.2 & 17823 \\ 16830 & 14.2 & 17072 \\ 10502 & 9.6 & 28685 \\ 12944 & 11.8 & 16526 \\ 13661 & 9.4 & 11450 \\ 15725 & 7.2 & 31541\end{array}$

5,450

18.9

COUNT Y

SAN PATRICO

औi.

\section{NTM-WELIS}

PLOR LIFT

$\begin{array}{rrr}\text { OIL-PRD } & \text { E/WD } & \text { GAS-PRD } \\ & & \\ 454 & 2.5 & 2126 \\ 2282 & 3.1 & 6471 \\ 2260 & 2.8 & 2358 \\ 4124 & 2.3 & 25413 \\ 2057 & 1.1 & 1359 \\ 5196 & 2.8 & 9230 \\ 21172 & 10.7 & 33758 \\ 30749 & 13.7 & 66389 \\ 52312 & 17.5 & 102885 \\ 70941 & 25.6 & 60575\end{array}$

$0.0 \quad$ C. 5

0.02 .0

0.02 .3

$0.0 \quad 4 . .3$

$0.0 \quad 5.0$

$0.0 \quad 5.0$

0.05 .4

$0.0 \quad 6.2$

$\begin{array}{ll}0.1 & 8.1 \\ 0.0 & 7.6\end{array}$
$70941 \quad 25.6$

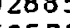


ABANDONED ONSHORE OIL FIELDS MITH OVER 250,000 BBL. CUMOLATIVE PRODOCTICH

\section{DST DISC ABD. PLD NOM PIELD NAHE}

$04 \quad 1942 \quad 1974 \quad 78123001$ ROORE

$\begin{array}{cccccl}\text { DEPTH } & \text { GRAVITI } & \text { ～CUHOL. PROD. OF FIELD } & \text { COONTY } \\ 7.135 & 39.1 & \cdots & 1,840,066 & \text { SAN PATRICO }\end{array}$

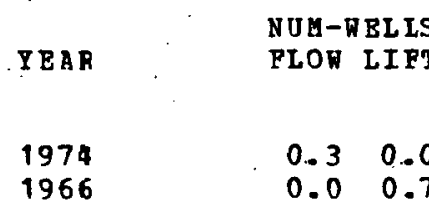

DEPTE GRAVITY

$5,446 \quad 29.8$
$1,840,066$

SAN PATRICO
NTM-MELLS

PLOR LIFT

$0.0 \quad 0.3$

0.02 .1

0.0 .4 .4

0.08 .5

0.011 .3

0.011 .7

$\begin{array}{rr}0.0 & 10.8 \\ 0.0 & 9.6\end{array}$
1970

1963

1967

1965
OIL-PRD E/WD

GA S-PRD

$\begin{array}{rr}772 & 8.5 \\ 3653 & 4.8 \\ 13369 & 8.3 \\ 22419 & 7.2 \\ 33669 & 8.1 \\ 55739 & 13.1 \\ 72216 & 18.4 \\ 64532 & 18.4\end{array}$

794 3311 10721 16817
33718 33718 102874 130957 
ABANDONED ONSACRE OIL FEELDS VITH OVER 250,000 BBL. CUMOLATIVE PRODUCTION

DST DISC ABD. PID. NOM FIELD NABE

041952197297708900 TILLYAN, NORTH (47C0)

DST DISC ABD. FLD NDM PIELD NAME

04.19551973 .72043742 PLYMOOTH (6200 A SAND)

DEPTH
4,750


IEAR

1972
1971
1970
1969
1968
1967
1966

$\begin{array}{cc}\text { DEPTH } & \text { GRAVITY } \\ 6.203 & 46.0\end{array}$

GR AV IT Y

30.0
NUA-REL LS

FLOW LIET

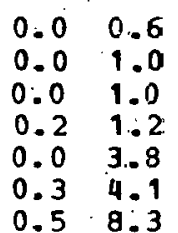

$\begin{array}{ll}0.0 & 0.6 \\ 0.0 & 1.0\end{array}$

$0.0 \quad 1.0$

0.03 .8

$0.5 \cdot 8.3$

COHOL. PROD. OP EIELD

885,645

921,234
CCONTI

SAN PATRICO

$\begin{array}{rrr}\text { OIL-PRD } & \text { E/HD } & \text { GAS-PRD } \\ & & \\ 200 & 0.9 & 3 \\ 2084 & 5.7 & 12 \\ 2297 & 6.3 & 12 \\ 2505 & 5.1 & 2671 \\ 6264 & 4.6 & 249554 \\ 6958 & 4.3 & 281958 \\ 8668 & 2.7 & \$ 3444\end{array}$

COUNTY

SAN PATRICO

\begin{tabular}{lll} 
& \multicolumn{2}{l}{ NOH-WELLS } \\
IEAR & FLON LIFT \\
& & \\
1973 & 0.0 & 0.2 \\
1972 & 0.0 & 1.0 \\
1971. & 0.0 & 1.0 \\
1970 & 0.0 & 1.4 \\
1969 & 0.0 & 2.0 \\
$1968^{\circ}$ & 0.0 & 2.4 \\
1967 & 0.0 & 3.0 \\
1966 & 0.0 & 4.4
\end{tabular}

$\begin{array}{rrr}\text { OIL-PRD } & \text { E/HD } & \text { GAS-PRD } \\ & & \\ 574 & 9.4 & 1427 \\ 4243 & 11.6 & 15280 \\ 5884 & 16.1 & 6067 \\ 6345 & 12.3 & 5606 \\ 8101 & 11.1 & 14193 \\ 14089 & 16.0 & 14315 \\ 20549 & 18.8 & \equiv 5267 \\ 18831 & 11.7 & 25838\end{array}$


ABANDONED ONSHORE OIL FIELDS NITH OVER 250,000 BBL. CUMOLATIVE PRODOCTION

NST DTSC ABD. FLD NTM FIRLD MAME

$04.19 .531971 \quad 73371952$ POERTO EAT (6750 SAYD)

\begin{tabular}{|c|c|c|c|}
\hline DEPTH & GRAV IT I & \multicolumn{2}{|c|}{ COHUL. } \\
\hline 6,746 & +2.0 & & \\
\hline YEAR & & $\begin{array}{l}\text { NOM- } \\
\text { ELOR }\end{array}$ & $\begin{array}{l}\text { EL IS } \\
\text { LI FT }\end{array}$ \\
\hline $\begin{array}{l}1971 \\
1970 \\
1969 \\
1968 \\
1967 \\
1966\end{array}$ & & $\begin{array}{l}0.8 \\
1.0 \\
0.9 \\
1.0 \\
0.8 \\
2.0\end{array}$ & $\begin{array}{l}1.3 \\
2.0 \\
2.0 \\
2.0 \\
3.3 \\
2.0\end{array}$ \\
\hline
\end{tabular}

DEPTH

5,800

\section{GRAV ITY}

40.0
DST DTSC ABD. FLD NUM FIELD NABE

041955197058271072 MATHIS, E. (CHOP ELAS SAND)
CUMOL. PROD. OP FIELD

764,542
COUNTY

SAN PATRICO
$850.2 \cup 8$

$\begin{array}{rrr}\text { OIL-PRD } & \text { E/RD } & \text { GAS-PRD } \\ & & \\ 913 & 1.2 & 3238 \\ 3890 & 3.6 & 10623 \\ 6866 & 6.4 & 19469 \\ 13725 & 12.5 & 34427 \\ 23978 & 16.4 & 53892 \\ 28140 & 19.3 & 67285\end{array}$

COOATY

SAN PATRICO

\begin{tabular}{lll} 
YEAR & \multicolumn{2}{c}{ NOM-RELIS } \\
& PLOR LI FT \\
& & \\
1970 & 0.0 & 1.0 \\
1969 & 0.0 & 2.0 \\
1968 & 0.0 & 2.6 \\
1967 & 0.0 & 3.9 \\
1966 & 0.0 & 4.0
\end{tabular}

\begin{tabular}{rrr} 
OIL-PRD & E/TD & \multicolumn{1}{r}{ GAS-PRD } \\
& & \\
942 & 2.6 & 21473 \\
3547 & 4.9 & 55306 \\
5856 & 6.2 & 98325 \\
13363 & 9.3 & 144058 \\
18415 & 12.6 & 60342
\end{tabular}


ABA NDONFD ONSHORE OIL FIEIDS ITH OVER 250,000 BBL. COMULATIVE FRODOCTICN

DST DISC ABD. FLD NTM PRELD NAME

041955197196979800 H.ITE POINT, E. (5950 SAND)

\section{DEPTH GRAVITY}

5.945

43.1

COHOL. FROD. OF FIELD

670,897

COONTY

SAN PATRICO

\begin{tabular}{lll} 
& \multicolumn{2}{l}{ NOM-HELIS } \\
YEAR & PLON IIFT \\
& & \\
1971 & 0.0 & 0.3 \\
1970 & 0.0 & 0.8 \\
1969 & 0.0 & 1.7 \\
1968 & 0.1 & 2.8 \\
1967 & 0.0 & 3.0 \\
1966 & 1.0 & 2.9
\end{tabular}

$\begin{array}{rrr}\text { CIL-PRD } & \text { E/RD } & \text { GAS-PRD } \\ & & \\ 334 & 2.7 & 2205 \\ 1332 & 4.9 & 22054 \\ 16566 & 27.2 & 21531 \\ 22946 & 21.6 & 36736 \\ 27235 & 24.9 & 33760 \\ 26319 & 18.4 & 28560\end{array}$

DST DISC ABD. PLD NTI PIELD NAUE

DEPTH GRAVITY

COMUL. EROD. OP PIELD

COONTY

$5,088 \quad 32.8$

645,904

SAN PATRICO

$\begin{array}{lr}\text { DEPTH } & \text { GRAVITY } \\ 4,914 & 32.0\end{array}$

COHUL. PROD: OP FIELD

CCONTY

623,466

SAN PATRICO

041952197541977333 HODGES (STILLEELI SAND, OP)

YEAR
1975
1974
1973
1972
1971
1970
1969
1968
1967
1966

NOH RELLS

FLON LIFT

$0.0 \quad 0.2$

0.01 .3

$0.0 \cdot 2.0$

0.02 .0

0.02 .4

0.43 .0

1.03 .0

0.83 .6

$\begin{array}{ll}1.0 & 5.0 \\ 1.0 & 5.0\end{array}$

\section{3,466}

$\begin{array}{rrr}\text { OIL-PRD } & \text { E/RE } & \text { GAS-PRD } \\ & & \\ 149 & 2.4 & 2 \\ 1311 & 2.9 & 116 \\ 2013 & 2.8 & 266 \\ 4539 & 6.2 & 24 \\ 7781 & 8.8 & 24 \\ 4360 & 3.5 & 2921 \\ 8656 & 5.9 & 20911 \\ 10878 & 6.9 & 51480 \\ 15853 & 7.2 & 96866 \\ 19553 & 8.9 & 116871\end{array}$


ABANDONED ONSHORE OIL PIELDS ITH OVER 250,000 BBL. COUOLATIVE FROdOCTION

DST DISC ABD. FID NOH PIRLD HABE

$04 \quad 19521974 \quad 93754714$ STNTON, NORTR (4700 SAND)

DEPTH
4,733

YE BR

1974
1973
1972
1971
1970
1969
1968
1967
1956

DE PTH

8,730

$\begin{array}{ll}\text { DST } & \text { DISC } \\ 04 \quad 1954\end{array}$

61154063 IDHAY (PRIO 8700 SAND)

DST DISC ABD. PLD NOM FIELD NAME

$0419651975 \quad 34990800$ GINNY, E. (7650)
GRAVIT Y

30.7

509,548

CCONTY

SAN PATRICO
GRAVIT Y

43.0

GRAVITY

7,645

42.8

COMOI. PROD. OF FIELD

$50 \cdot 3,438$
OIL-PRD F/MD GAS-PRD

$\begin{array}{ll}0.0 & 0.4 \\ 0.0 & 1.0 \\ 0.0 & 0.9 \\ 0.0 & 1.0 \\ 0.0 & 1.0 \\ 0.0 & 2.2 \\ 0.0 & 2.8 \\ 0.0 & 3.2 \\ 0.0 & 4.5\end{array}$

COHOL. PROD. OP PIELD

507,867

$\begin{array}{rr}491 & 3.2 \\ 1927 & 5.3 \\ 2499 & 7.5 \\ 1644 & 4.5 \\ 2202 & 6.0 \\ 5844 & 7.4 \\ 7435 & 7.4 \\ 10730 & 9.3 \\ 18269 & 19.1\end{array}$

784

9759

12013

5188

5846

12581

14734

25369

37898
Con NTY

SAN PATRICO

COONTY

SAN PATRICO

\begin{tabular}{lll} 
& \multicolumn{2}{c}{ NOH- WELIS } \\
YEAR & \multicolumn{2}{c}{ FLON LIPT } \\
& & \\
1975 & & \\
1974 & 0.8 & 0.0 \\
1973 & 2.6 & 0.0 \\
1972 & 3.0 & 0.0 \\
1971 & 3.0 & 0.0 \\
1970 & 3.7 & 0.0 \\
1969 & 4.0 & 0.0 \\
1968 & 4.0 & 0.0 \\
1967 & 4.0 & 0.0 \\
1966 & 4.0 & 0.0 \\
& 3.0 & 0.0
\end{tabular}

$\begin{array}{rrr}\text { OIL-FRD } & \text { E/HD } & \text { GAS-PRD } \\ & & \\ 12685 & 41.7 & 12629 \\ 17352 & 18.4 & 17856 \\ 21005 & 19.2 & 22314 \\ 29664 & 27.1 & 36696 \\ 46114 & 34.5 & 60478 \\ 58528 & 40.1 & 69605 \\ 66096 & 45.3 & 88960 \\ 74280 & 50.9 & 104549 \\ 75369 & 51.6 & 73907 \\ 67812 & 61.9 & 63437\end{array}$


ABANDONED ONSHORE OIL PIELDS ITT ? ?R 250,000 BBL COMULATIVE PRODUCTICH

DST DISC ABD. PLD NOM PIELD NAHE

041955197072043702 PLYMODTH $\cdot(6150$ SAND)

\begin{tabular}{|c|c|c|c|}
\hline DE PTH & GRAVITY & \multicolumn{2}{|c|}{ COUE } \\
\hline 6,118 & 41.0 & & \\
\hline YEAE & & $\begin{array}{l}\text { NOM- } \\
\text { FLOR }\end{array}$ & $\begin{array}{l}\text { IL IS } \\
\text { LI PT }\end{array}$ \\
\hline $\begin{array}{l}1970 \\
1969 \\
1968 \\
1967 \\
1966\end{array}$ & & $\begin{array}{l}0.0 \\
0.0 \\
0.0 \\
0.0 \\
0.5\end{array}$ & $\begin{array}{l}0.2 \\
1.4 \\
1.0 \\
1.0 \\
0.5\end{array}$ \\
\hline
\end{tabular}

DEPTH

6,110

\section{GRAVITY}

41.0
COMUL. PROD. OF FIELD

476,271
COONTY

SAN PATRICO

$0419581976 \quad 80331333$ SAN PATRICIO (VICKSBORG 6100)

\begin{tabular}{|c|c|c|}
\hline YEAR & $\begin{array}{l}\text { NDM- } \\
\text { PLOR }\end{array}$ & $\begin{array}{l}\text { ELIS } \\
\text { I I FT }\end{array}$ \\
\hline $\begin{array}{l}1976 \\
1975 \\
1974 \\
1973 \\
1972 \\
1971 \\
1970 \\
1969 \\
1969 \\
1967 \\
1966\end{array}$ & $\begin{array}{l}0.0 \\
0.0 \\
0.0 \\
0.0 \\
0.0 \\
0.0 \\
0.0 \\
0.0 \\
0.0 \\
0.0 \\
0.0\end{array}$ & $\begin{array}{l}0.3 \\
0.3 \\
0.9 \\
0.03 \\
1.3 \\
1.0 \\
2.0 \\
3.0 \\
3.10 \\
3.0 \\
3.0\end{array}$ \\
\hline
\end{tabular}

$\begin{array}{rrr}\text { OIL-PRD } & \text { E/WD } & \text { GAS-PRD } \\ & & \\ 616 & 2.0 & 26 \\ 761 & 2.5 & 73 \\ 719 & 2.1 & 70 \\ 740 & 2.4 & 71 \\ 488 & 1.3 & 42 \\ 1056 & 2.9 & 98 \\ 1703 & 1.8 & 146 \\ 3931 & 3.6 & 386 \\ 6048 & 5.5 & 595 \\ 7773.5 & 7.1 & 964 \\ 9977 & 9.1 & 2516\end{array}$

$\begin{array}{rcr}\text { OII-PRD } & \text { E/RE } & \text { GAS-PRD } \\ & & \\ 826 & 13.6 & 206 \\ 8824 & 17.1 & 7191 \\ 4792 & 13.1 & 5923 \\ 7762 & 21 . .2 & 6557 \\ 4903 & 13.4 & 14183\end{array}$

9977

COONTY

SAN PATRICO 
ABANDONED ONSHORE OIL PIEIDS ITH OVER 250,000 BBL. CUMOLATIVE PRODUCTICN

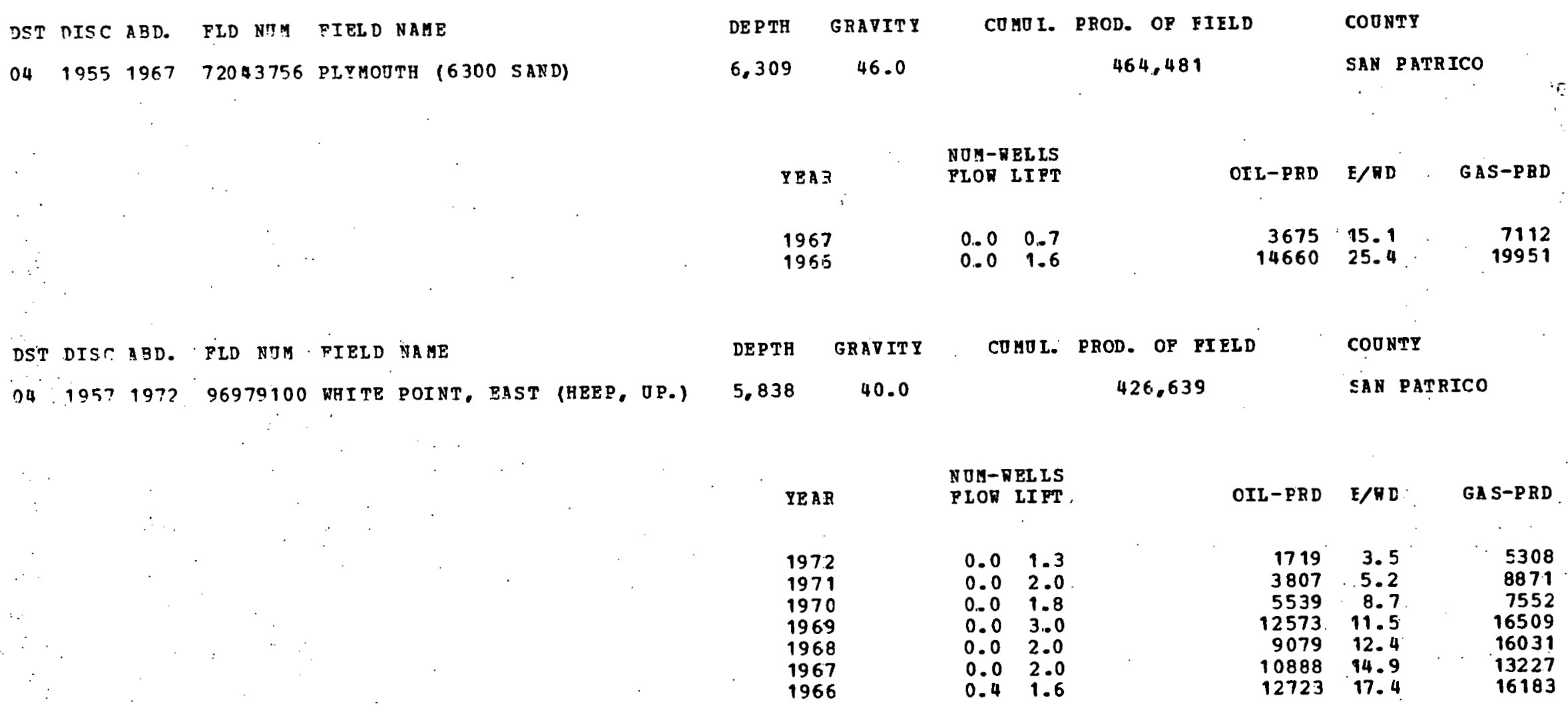


ARANDONED ONSHORE OEL FIELDS HTH OVER 250,000 BBL. CUMULATIVE PRODUCTION

DST DISC ABD. FLD NOM PIELD NAME

$04 \quad 1951 \quad 196783754850$ SINTON, NORTH (5700 SAND)

EST DIS: ARD. FID NCM FIELD NAHE

$04 \quad 19551970 \quad 72043738$ PLYMOJTH (6200 SAND)

\begin{tabular}{|c|c|c|c|}
\hline DEPTH & GRAVITY & CUMOI. PROD. OF FIELD & COONTY \\
\hline 5,785 & 35.4 & 387,749 & SAN PATRICO \\
\hline
\end{tabular}

\begin{tabular}{|c|c|c|c|c|}
\hline YEAB & $\begin{array}{l}\text { NOM-WELIS } \\
\text { PLOW IIPT }\end{array}$ & OIL-PQD & $E / N D$ & GAS-PRD \\
\hline $\begin{array}{l}1967 \\
1966\end{array}$ & $\begin{array}{ll}0.0 & 0.6 \\
0.0 & 1.0\end{array}$ & $\begin{array}{l}1199 \\
4602\end{array}$ & $\begin{array}{r}5.6 \\
12.6\end{array}$ & $\begin{array}{l}5413 \\
5968\end{array}$ \\
\hline
\end{tabular}

$\begin{array}{ccccl}\text { DEPTH } & \text { GRAVITY } & \text { COMOL } & \text { PROD. OF FIELD } & \text { COUNTY } \\ 6,188 & 42.0 & 332,5 E 4 & \text { SAN PATRICO }\end{array}$

YEAR

NOH-RELIS

PLON LIFT

\begin{tabular}{rrr} 
OIL-PRD & E/ND & GAS-PRD \\
& \multicolumn{1}{c}{} \\
723 & 4.8 & 1955 \\
8969 & 24.6 & 5831 \\
10565 & 28.9 & 9654 \\
6838 & 18.7 & 7112 \\
4265 & 6.4 & 8051
\end{tabular}


ARANDONED ONSHORE OIL FIEIDS WITH OتER 250,000 BEL. CUMOLATIVE PRODUCTICN

DST DTSC ABD. FLD NOM FIELD NAME

$04 \quad 19591970 \quad 72043846$ PLYMOUTH (6620 SAND)

DST DISC AED. FLD NUM FIELD NAME

$04 \quad 1953$

88129945 TAFT, SOUTH (7200 SAND)

DST DISC ABD. FID NOI FIELD NAME

$04 \quad 1951$

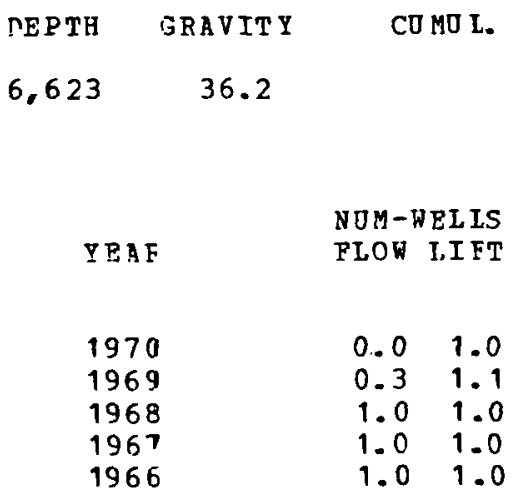

DEPTH GRAVITY

7,185

43.7

DEPTH GRAVITY

CDMOI. EROD. OF FIELD

315,543

$6,824 \quad 40.4$

325.476
COUNTY

SAN PATRICO

$\begin{array}{rrr}\text { OIL-PRD } & \text { E/RD } & \text { GAS-PRD } \\ & & \\ 2603 & 7.1 & 4768 \\ 12460 & 24.1 & 26119 \\ 7970 & 10.9 & 8723 \\ 12854 & 17.6 & 24700 \\ 17479 & 23.9 & 36002\end{array}$

$\operatorname{CONNTY}$

SAN PATRICO

COUNTY

SAN PATRICO 
ABA NDONED ONSHORE OIL FIRLDS NITH OVER 250,000 BBL. COMULATIVE PRODUCTICN

DST DISC ABD. PLD NHM PIBLD NAME

041950197583754068 SINTON, NORTH (GFTSER SAND)

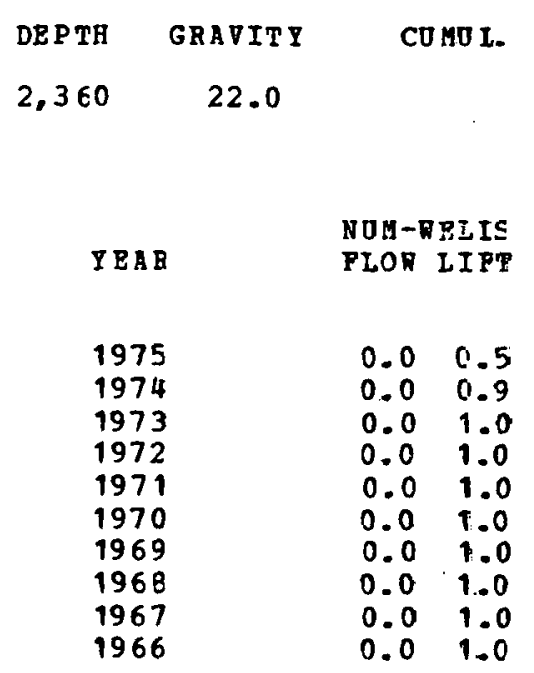

DEPTH

GRA ITT Y

4,124

21.7

88132498 T4PT, REST (4100 SAND)

PID NTM FIEId NauE

DST DISC ABD.

$04 \quad 1954$
DEPTE GRAVITY

6,114

41.0
COMO1. PROD. OF FIELD

263,373
COUNTY

SAN PATRICO

$$
\text { SAN PATRICO }
$$

E/MD GAS-PRD

$\begin{array}{rr}3.9 & 1254 \\ 4.8 & 3027 \\ 5.2 & 5350 \\ 7.3 & 11697 \\ 7.3 & 4021 \\ 8.4 & 15762 \\ 4.8 & 15878 \\ 7.5 & 10656 \\ 10.5 & 8864 \\ 12.5 & 7857\end{array}$

CODNTI

SAN PATRICO

CCONT

SAN PATRICO 
ABANDONED ONSHORE OIL FIELDS WITH OVER 250,000 BBL. CUMULAT IVE PRODUCT ION

DST NTSC ARD. FLD NOM PIEID NAME

$0419561966 \quad 57883400$ MARY BLONTZEP (6 100 -B- SD.)
DEPTH

6,124

YRA

1966
GRAV IT Y

44.0

$$
\text { NOM-MELIS }
$$$$
\text { PLON LIPT }
$$

0.21 .8
CCONTY

SAN PATRICO

256,369

$\begin{array}{rrr}\text { OIL-PRD } & \text { E/AD } & \text { GAS-PRD } \\ 4670 & 6.4 & 3\end{array}$


ABANDONED ONSHORE OIL FIELDS VITH OVER 250,000 BBL. COHULATIVE PRODOCTICA

DST DISC ABD. PID NOM FIRLD NAME

Q4 $1933 \quad 1973 \quad 37300001$ GTERRA

DST DISC ABD. PLD WOM PIELD NAME

O4. 1941 1973, 99549001 YTDRRIA

$\begin{array}{ccccc}\text { DEPTH } & \text { GRAVITY } & \text { CORDL. PROD. OP PIELD } & \text { GOORTY } \\ 2,245 & 34.5 & 3.791,988 & \text { STARR }\end{array}$

YEAF
1973
1972
1971
1970
1969
1968
1967
1966

NOH-RELIS

OIL-PRD E/HD GAS-PRD

$0.0 \quad 1.0$

$0.0 \quad 1.0$

0.01 .0

0.02 .0

0.02 .3

$0.0 \quad 2.1$

$0.8 \quad 2.2$

$1.0 \quad 2.0$

$\begin{array}{rr}2126 & 5.8 \\ 2251 & 6.2 \\ 2798 & 7.7 \\ 4340 & 5.9 \\ 8842 & 10.4 \\ 11712 & 15.4 \\ 13803 & 12.6 \\ 12869 & 11.8\end{array}$

943

1778

2311

6476

5123
11705

73834

130126

$\begin{array}{cccc}\text { DEPTH } & \text { GRAVITY } & \text { CIIUT. PROD. OF PIELD } & \text { CCORTY } \\ 4,225 & 43.3 & 2,905,289 & \text { STRRR }\end{array}$

YEA R

NOS-HELIS

PLON IIFT

CIL-PRD E/RD

GAS-PBD

1973

1972

1971

1970

1969

1968

1967

$\begin{array}{ll}0.0 & 0.9 \\ 0.0 & 2.6 \\ 0.0 & 3.0 \\ 0.0 & 3.0 \\ 0.0 & 3.3 \\ 0.0 & 4.0 \\ 0.0 & 3.7 \\ 1.2 & 4.5\end{array}$

$0.0 \quad 0.9$

$\begin{array}{ll}0.0 & 2.6 \\ 0.0 & 3.0\end{array}$

$0.0 \quad 3.0$

0.04 .3

$\begin{array}{ll}0.6 & 3.7 \\ 1.2 & 4.5\end{array}$

$\begin{array}{rr}744 & 2.2 \\ 3952 & 4.2 \\ 5036 & 4.6 \\ 5122 & 4.7 \\ 5616 & 4.7 \\ 8712 & 6.0 \\ 12297 & 7.9 \\ 17142 & 8.3\end{array}$

9
28
36
48
02
74
66
87


ABANDONED ONSHORE OIL FIELDS WITA ORER 250,000 BBL, COMOLATIVE PRODUCTICN

DST DISC ABD. FLD NOM FIELD NAME

$0419401972 \quad 11096001$ BOYLE

DST DISC ABD. FID NOM FIELD NAME

$04 \quad 1947 \quad 1970 \quad 23893001$ DEE DAVENPORT
DE PTH
GRAVITY
3,500
44.0
COMOI.
$2,408,693$
COONTY

YEAR
1972
1971
1970
1969
1968
1967
1966

$\begin{array}{cc}\text { NOM-WELIS } \\ \text { FLON } \\ \\ \\ 0.9 & \\ 0.4 & 1.0 \\ 0.4 & 1.5 \\ 0.0 & 1.7 \\ 0.0 & 2.0 \\ 0.0 & 3.7 \\ 0.0 & 4.3 \\ 0.0 & 4.7\end{array}$

OIL-PRD E/WD

$1088 \quad 1.6$

$450 \quad 0.6$

$2068 \quad 3.4$

1059 1.5

$1618 \quad 1.2$

$4213 \quad 2.7$

$5572 \quad 3.3$

GAS-PRD

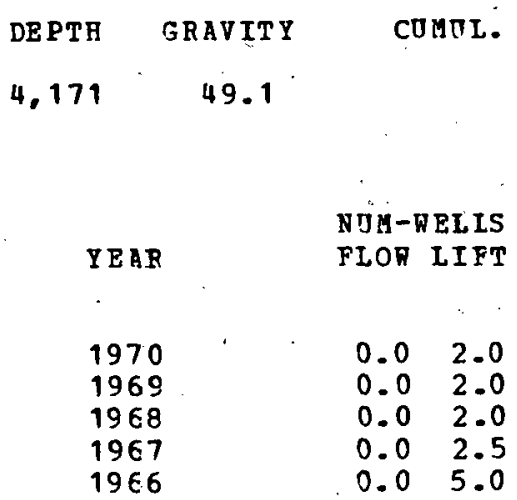

ROD. OF FIELD

$1,307.232$
COONTY

STARR
. 5.0

$\begin{array}{rr}\text { OI.L-PFD } & E / H C \\ & \\ 2093 & 2.9 \\ 4243 & 5.8 \\ 5230 & 7.2 \\ 7241 & 7.9 \\ 19046 & 10.4\end{array}$

GAS-PRD

1014

1426

1400

4903 44499 
ARANDONFD ONSHORE OIL PIEIDS VITH OVER 250,000 B8L. CUMULATIVE PRODUCTION

DST DISC ABD. FLD NOM FIELD NAME

$04 \cdot 19481972 \quad 10711001$ BOROSA

DST DISC ABD. PLD NOT PIELD NAME

$0419481970 \quad 50700001$ LA COPITA

$\begin{array}{ccccc}\text { DEPTH } & \text { GRATITY } & \text { COMOL. } & \text { PROD. OF FIELD } & \text { COONTY } \\ 4.273 & 47.6 & 821,004 & \text { STARR }\end{array}$

YEAR

NOM-RELIS

PLON LIPT

E/WE

GAS-FRD

1972

1970

1969

1968

1967

0.80 .9

0.32 .4

1. 04.6

1.05 .8

1.05 .9

$9.6 \quad 5.8$

1478

$\begin{array}{ll}1478 & 2.3 \\ 1799 & 1.8\end{array}$

$1799 \quad 1.8$

$6144 \quad 3.0$

$11202: 4.5$

127875.1

$192315 \quad 34.2$

20035

8620

8620
51854

$\mathbf{7} 956$
57936

$\begin{array}{r}57936 \\ \hline\end{array}$

274144
DEPTH GRAVITY COMUI. PROD. OF FIELD COUNTY
6,950
40.8
657,250
STA BR

$\begin{array}{lll} & \text { NOL-VELLS } \\ \text { PEAR } & \text { FLON LIFT } \\ & & \\ 1970 & 0.0 & 0.4 \\ 1969 & 0.0 & 1.0 \\ 1968 & 0.0 & 1.0 \\ 1967 & 0.0 & 1.0 \\ 1966 & 0.1 & 1.2\end{array}$

$\begin{array}{rrr}\text { OIL-PED } & \text { E/KE } & \text { GAS-PBD } \\ & & \\ 706 & 4.6 & 950 \\ 1983 & 5.4 & 2939 \\ 2116 & 5.8 & 2495 \\ 2730 & 7.5 & 3785 \\ 2409 & 5.3 & 4455\end{array}$


A3ANDONED ONSHORE OII PIELDS ITH CVEB 250,000 BEL. CUMULATITE PAODUCTION

DST DISC ABD. FLD NDM FIELD NAME

04. 1932197376842001 RIO GRANDE CITY

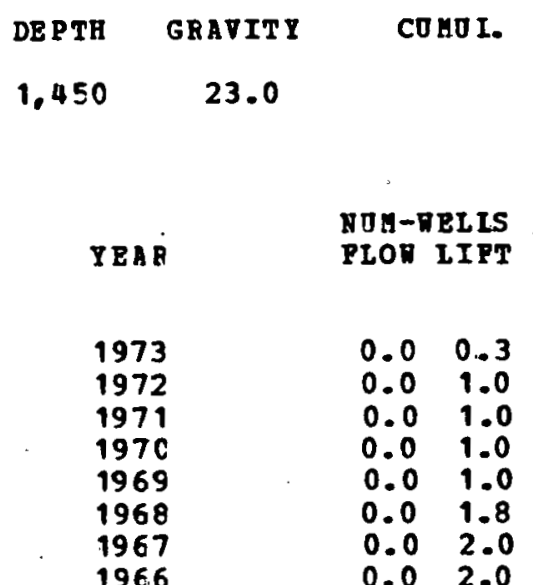

DEPTB GRAVITY

$5,695 \quad 43.8$
COMOL. PROD. OP FIELD

530,122
COONTI

STARR
DST DISC ABD. PID NTE PIELD VALE

04. 1953196727905369 PL BENADITO (KEBIETT SAND)

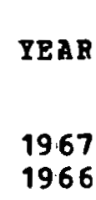


ABANDONED ONSHORE OIL FIELDS RITH OVER 250,000 BBL. COUOLATIVE PROLOCTION

DST NTSC ATD. PI.D NDM FIRLD HAME

041952197327905451 EL BENADITO (ZANARINE)

NST DISC ABD.

041960

87124731 SUN, NORTH $(4950$ SAED)

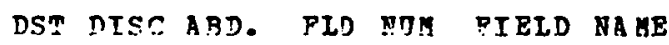

$04 \quad 1948 \quad 1968 \quad 19141001$ COASTAL

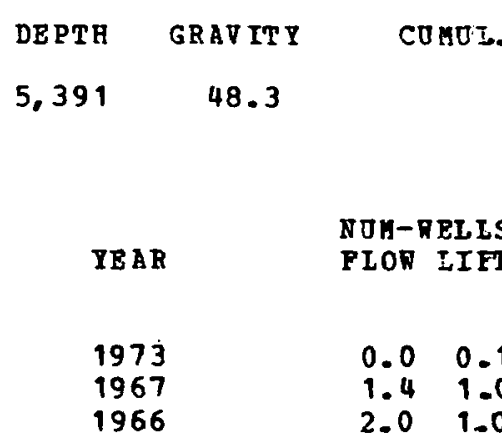

GRAVIT Y

4,953

47. 0

DE PT H

5.750
GRAVITY

42.5

COMOL: PROD. OF FIELD

384.573

NOM-DELIS

PLOR LIPI

YEAR

1968

1967

1966

$$
\begin{array}{ll}
0.5 & 0.0 \\
1.0 & 0.0 \\
1.0 & 0.0
\end{array}
$$

468,743

CCUNTY

STA BR

$\begin{array}{rrr}\text { OIL - PRD } & \text { E/RD } & \text { GAS-PRD } \\ & & \\ 52 & 1.7 & 1 \\ 933 & 1.1 & 17447 \\ 1899 & 1.7 & 54702\end{array}$

431,112

COONTY

STARR

CCOATY

STARR

$\begin{array}{rlr}\text { OIL PRD } & \text { E/RD } & \text { GAS-P.RD } \\ & & \\ 95 & 0.5 & 868 \\ 2066 & 5.7 & 18892 \\ 1106 & 3.0 & 10089\end{array}$


ABANDONED ONSHORE OIL FIELDS NITR OVER 250,000 BBL . CUUULATIVE PRODUCTIGN

DST DISC ABD. FID NTM FIELD NAHE

$04 \quad 1955 \quad 1970 \quad 11504090$ RRAOLIA (MILLER)

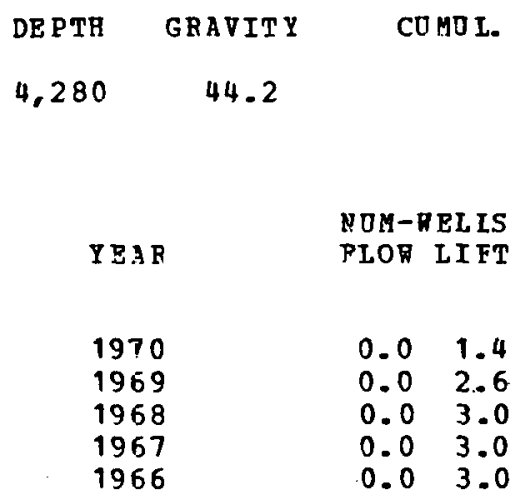

DE PTH

4,056

\section{GRAVIT Y}

41.6
COMOL.- PROD. OF FIELD

366,779
COONTY

STARR

\footnotetext{
041953197476723744 RINCON, NORTH (4060 SAND)
}

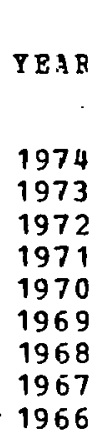

\begin{tabular}{lll} 
& \multicolumn{2}{l}{ NOH-QELIS } \\
YEAR & PLOR IIFT \\
& & \\
1974 & 0.0 & 0.7 \\
1973 & 0.0 & 1.00 \\
1972 & 0.0 & $1 . .7$ \\
1971 & 0.0 & 3.0 \\
1970 & 0.0 & 3.0 \\
1969 & 0.0 & 3.0 \\
1968 & 0.0 & 3.0 \\
1967 & 0.0 & 3.0 \\
1966 & 0.0 & 3.0
\end{tabular}

367,460

$\begin{array}{rrr}\text { CII_PRD } & \text { E/RD } & \text { GAS-PRD } \\ & & \\ 1037 & 2.0 & 1060 \\ 2975 & 3.2 & 896 \\ 6032 & 5.5 & 1360 \\ 10816 & 9.9 & 1889 \\ 14628 & 93.4 & 771\end{array}$

$\operatorname{CONNT}$

ST A RR

$\begin{array}{rrr}\text { OIL-PRD } & \text { E/RD } & \text { GAS-PRD } \\ & & \\ 413 & 1.7 & 956 \\ 942 & 2.6 & 1348 \\ 1378 & 2.3 & 1349 \\ 1425 & 1.3 & 1466 \\ 2169 & 2.0 & 2779 \\ 4137 & 3.8 & 4392 \\ 5826 & 5.3 & 4262 \\ 5719 & 5.2 & 4372 \\ 8503 & 7.8 & 5230\end{array}$


ABANDONED ONSBORE OII FIELDS DITH OVER 250,000 BBL. CUMOLATIVE PRODOCTION

DST DISC ABD. FID NUM PTELD NAGE

$04: 1963197636799496$ GRRGG DOOD, S. (3950)

DST DISC ABD. FLD HOM FIELD NAHE

$04.1965197348635084 \mathrm{KPISEY}$, SOOTR (ZCNE 16-A)
DEPTH

3,937

GRAV IT Y

CUMUL. PROD. OP PIELD

$\operatorname{CCONTI}$

STA RR

\begin{tabular}{lll} 
& \multicolumn{2}{c}{ YUY-TELLS } \\
YEAR & PLOR & IIFT \\
& & \\
1976 & 1.3 & 2.7 \\
1975 & 1.8 & 3.8 \\
1974 & 2.0 & 4.2 \\
1973 & 2.0 & 4.8 \\
1972 & 2.0 & 5.0 \\
1971 & 2.7 & 5.2 \\
1970 & 4.9 & 3.9 \\
1969 & 6.8 & 2.3 \\
1968 & 7.0 & 1.0 \\
1967 & 6.9 & 1.1 \\
1966 & 1.6 & 5.7
\end{tabular}

358,714

DEPTH GRAVITY COUOL. PROD. OP FIELD COONTY

5.295

45.6

329,883

STA RR

\begin{tabular}{lll} 
& \multicolumn{2}{c}{ MOM- WELLS } \\
YEAR & FLN LIFT \\
& & \\
1973 & 0.0 & 0.3 \\
1972 & 0.0 & 1.0 \\
1971 & 0.0 & 1.0 \\
1970 & 0.2 & 1.3 \\
1969 & 1.8 & 0.3 \\
1968 & 2.0 & 0.0 \\
1967 & 2.7 & 0.0 \\
1966 & 3.0 & 0.0
\end{tabular}

$\begin{array}{rrr}\text { OIL-PRD } & \text { E/RE } & \text { GAS-PRD } \\ & & \\ 90 & 1.0 & 180 \\ 3779 & 10.3 & 4328 \\ 6629 & 18.2 & 25903 \\ 17528 & 33.9 & 25222 \\ 34487 & 47.2 & 26099 \\ 25468 & 34.9 & 41801 \\ 53071 & 54.5 & 98549 \\ 916067 & 1 \text { C6.0 } & 124218\end{array}$

GA S-P BD

$\begin{array}{rrr}134 & 0.1 & 8 \\ 991 & 0.5 & 284 \\ 1821 & 0.8 & 1610 \\ 2290 & 0.9 & 1796 \\ 4799 & 1.9 & 3540 \\ 5399 & 1.9 & 6575 \\ 12540 & 3.9 & 184843 \\ 25002 & 7.6 & 293772 \\ 35205 & 12.1 & 210946 \\ 43589 & 14.9 & 296070 \\ 52293 & 19.8 & 208279\end{array}$

8

1610

6575

93772

10946

208279 
ABANDONED ONSHORE OIL PIEIDS VITH OFER 250,000 BBL. CUMULATIVE FRODUCTION

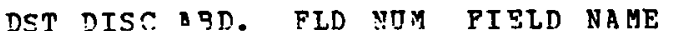

ก4 $19581970 \quad 97124638$ STN, NORTH (SAENZ - D-)

DST.DISC QDD. FID NTYY FIELD NAME

04. $1959.1976 \quad 76729568$ RINCON, NEST (3500 PB C)

\begin{tabular}{|c|c|c|c|}
\hline EPTH & GRAV IT Y & \multicolumn{2}{|c|}{ COMOL. } \\
\hline 5,349 & 45.6 & & \\
\hline YFAR & & $\begin{array}{l}\text { NOH- } \\
\text { PIOD }\end{array}$ & $\begin{array}{l}\text { EL LS } \\
\text { II PT }\end{array}$ \\
\hline $\begin{array}{l}1970 \\
1969 \\
1968 \\
1967 \\
1966\end{array}$ & & $\begin{array}{l}0.0 \\
0.0 \\
0.0 \\
0.0 \\
3.0\end{array}$ & $\begin{array}{l}9.0 \\
2.0 \\
2.0 \\
2.03 \\
0.4\end{array}$ \\
\hline
\end{tabular}

1966 .

$\begin{array}{cc}\text { DEPTH } & \text { GRAVITY } \\ .3 .541 & 38.5\end{array}$

COMOL. PROD. OF FIELD

OIL-PRD. E/WD GAS-PRD

$\begin{array}{rrr}195 & 0.5 & 6754 \\ 1729 & 2.4 & 5718 \\ 3617 & 5.0 & 12778 \\ 4295 & 5.0 & 21312 \\ 9825 & 7.9 & 69633\end{array}$

98257.9 .69633

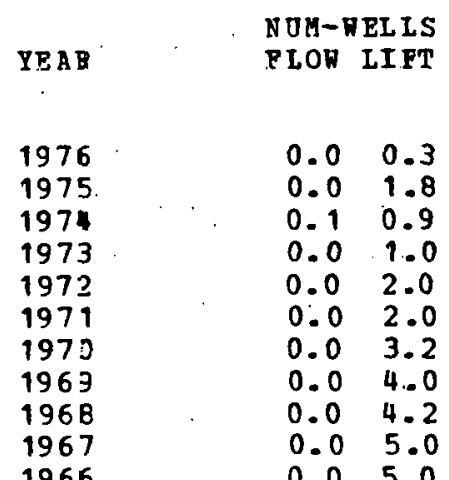

270,837

287,718

CCONTY

STARA

CODNTI

STA BR

$\begin{array}{rrr}\text { OII-PRD } & \text { E/RD } & \text { GAS-PRD } \\ & & \\ 43 & 0.5 & 1 \\ 913 & 1.4 & 12 \\ 4537 & 12.4 & 12 \\ 7253 & 19.9 & 12 \\ 11517 & 15.8 & 19 \\ 7285 & 10.0 & 24 \\ 3392 & 2.9 & 190 \\ 4014 & 2.7 & 2226 \\ 4932 & 3.2 & 2402 \\ 5285 & 2.9 & 1916 \\ 8081 & 4.4 & 616\end{array}$


ABA NDONED ONSHORE OII FIEIDS VITH OVER 250,000 BBL. COHOLATIVE PRODUCTICN

DST DISC ABD. FLD NTM FTELD NAME

$04 \quad 1949 \quad 1967 \quad 13033001$ SกISVA VISTA

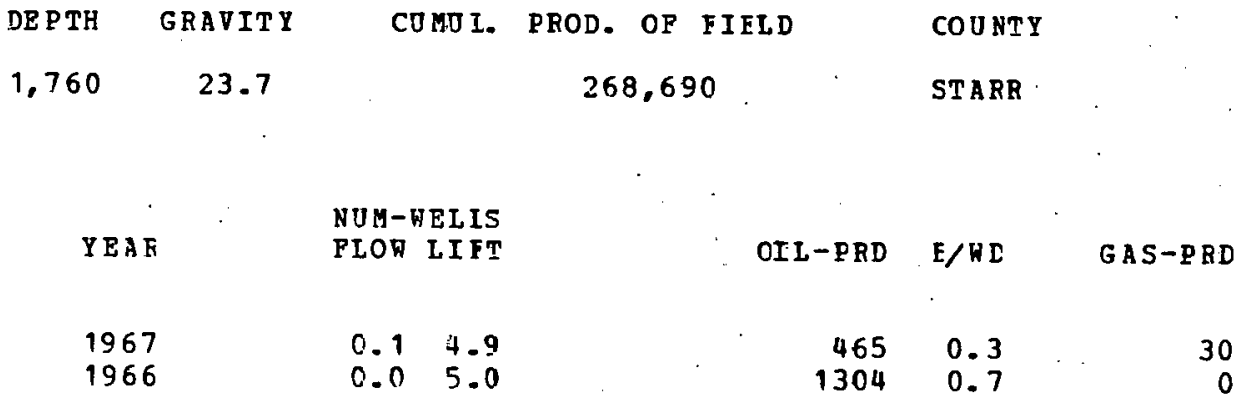

18 PIELD (S)
$16,509,490$ COMULATIVE PROD. FROM ABANDONED PIELDS 
ABANDONED ONSHORE OII FIELDS NITH ORER 250,000 BBL. CUMOLATIVE FRODDCTION

DST DISC ABD. PLD NOM PIELD NABE

0419289943 . 1186001 ALBRRCAS

NST DISC ABD. PLD NOM PIELD MALE

O4 $19521972 \quad 37514500$ GOTIORREZ (ROSENERRG SAND)

DST DISC ABD. FLD NOM FIELD NAME

$04 \quad 1946 \quad 1971 \quad 43319001$ н ORHES

\begin{tabular}{|c|c|c|c|c|}
\hline DEP TH & SRAVITY & conol. & PROD. OF EIELD & COONTY \\
\hline 2,150 & 21.0 & & $2,544,614$ & DEBE \\
\hline & & & & \\
\hline DEPTR & GRAVITY & CUnOL. & PROD. OF FIEID & COUNTY \\
\hline 1,667 & 25.0 & & $1.611,240$ & NEAP \\
\hline
\end{tabular}

IEAE:

VUR-RELIS FIOR IIPT

$$
\text { OIL-PRD E/RD }
$$

GA S-P RD

1972

1979

1970

1969

1968
1967

1966

$\begin{array}{rr}0.0 & 0.9 \\ 0.0 & 2.6 \\ 0.0 & 26.7 \\ 0.0 & 30.0 \\ 0.0 & 30.0 \\ 0.0 & 30.0 \\ 0.0 & 30.0\end{array}$

$\begin{array}{rr}394 & 1.2 \\ 1245 & 1.3\end{array}$

$\begin{array}{ll}1245 & 1.3 \\ 1662 & 0.2\end{array}$

$2766 \quad 0.3$

$13912 \quad 1.3$

$21088 \quad 1.9$

$19254 \quad 1.8$

COONTY

DEPTH

GRAVITI

CU UOL. PROD. OP FIELD

805.711

TEP.
NOM-WELIS

FLOW IIFT

$0.0 \quad 6.7$

$0.0 \quad 10.0$

0.010 .0

$0.0 \quad 10.3$

$0.0 \quad 11.0$

0.011 .0
OIL-PRD E/RD

GA S-PRD 
ABANDONED ONSHORE OIL FIELDS WITH OVER 250,000 BBL. COMOLAT IVE PRODUCTION

DST DISC ARD. FLD NUM FIELD NAME

04194519721198001 ALBERCAS, NORTH

DST DISC ABD. FID NOM FIELD NAME

$04 \quad 1932 \quad 52299001$ LAUREL

DST DISC ABD. EID NOM EIELD NAME

0419511975 \$8188568 TAL VEZ (ROSENBERG, MI. SD.)

$\begin{array}{cc}\text { DEPTH } & \text { GRAVITY } \\ 2,313 & 24.0\end{array}$

CUMUL. PROD. OF FIEID

639,307

$\operatorname{CCDNTI}$

REBE

\begin{tabular}{lll} 
& \multicolumn{2}{c}{ NUM-WELLS } \\
YEAR & FLOW & LIFT \\
& & \\
1972 & 0.0 & 1.3 \\
1971 & 0.0 & 2.0 \\
1970 & 0.0 & 2.7 \\
1969 & 0.0 & 3.5 \\
1968 & 0.0 & 5.0 \\
1967 & 0.0 & 5.0 \\
1966 & 0.0 & 5.0
\end{tabular}

EEPTH GRAVITY

COMUL. RROD. OF FIELD

628,339

OIL-PRD E/ND

GAS-PRD

2,250

49.0

DEPTH

GRAVITY

1,975

35.4

COMOL. PROD.. OP PIELD

619,585

$\begin{array}{rr}738 & 1.5 \\ 1533 & 2.1 \\ 2064 & 2.1 \\ 3206 & 2.5 \\ 4009 & 2.2 \\ 4399 & 2.4 \\ 4789 & 2.6\end{array}$

14
24
24
24
24
24
0

COONTY

LEค

$\operatorname{COUNTY}$

EEB

\begin{tabular}{lll} 
& \multicolumn{2}{c}{ NINM-WELIS } \\
YEAR & FLOW & LI FT \\
& & \\
1975 & 0.0 & 1.5 \\
$197 \equiv$ & 0.0 & 0.4 \\
1972 & 0.0 & 1.0 \\
1971 & 0.0 & 1.0 \\
$197 C$ & 0.0 & 1.4 \\
1969 & 0.0 & 2.7 \\
1968 & 0.0 & 2.0 \\
1967 & 0.0 & 1.9 \\
1966 & 0.0 & 5.0
\end{tabular}

GA S-PRD

$\begin{array}{rr}582 & 1.1 \\ 358 & 2.4 \\ 1032 & 2.8 \\ 2445 & 6.7 \\ 8272 & 16.0 \\ 13692 & 13.6 \\ 11962 & 16.4 \\ 10633 & 15.2 \\ 8718 & 4.8\end{array}$


ABANDONED ONSHORE OIL FIEIDS VITH OVER 250,000 BBL. CUHOLATIVE PRODUCTICN

DST DISC ABD. FLD HOM PIELD NAME

$04 \quad 1950 \quad 1972 \quad 8187001$ RIILINGS

DST DISC ABD. FLD NOM PIELD NAME

$04 \quad 1926$

\begin{tabular}{|c|c|c|c|}
\hline DEPTH & GRAVITY & \multicolumn{2}{|c|}{ COU } \\
\hline 2,389 & 31.9 & & \\
\hline YEAR & & $\begin{array}{l}\text { NOY- } \\
\text { PLOR }\end{array}$ & $\begin{array}{l}\text { ELIS } \\
\text { LIPT }\end{array}$ \\
\hline $\begin{array}{l}1972 \\
1971 \\
1970 \\
1969 \\
1968 \\
1967 \\
1966\end{array}$ & & $\begin{array}{l}0.1 \\
1.1 \\
0.3 \\
0.0 \\
0.0 \\
0.1 \\
0.0\end{array}$ & $\begin{array}{l}0.9 \\
0.3 \\
1.07 \\
2.0 \\
3.0 \\
4.0 \\
3.7\end{array}$ \\
\hline
\end{tabular}

DEPTH GRAVITY

$5,050 \quad 34.0$

8 PIEID (S)
COUNTY

581,950

AREE

$\begin{array}{rrr}\text { OIL-PRD } & \text { E/RD } & \text { GAS-PRD } \\ & & \\ 449 & 1.2 & 11 \\ 1042 & 2.0 & 12 \\ 1534 & 2.1 & 12 \\ 2340 & 3.2 & 12 \\ 3110 & 2.8 & 20 \\ 3358 & 2.3 & 35 \\ 4021 & 3.0 & 0\end{array}$

COTNTY: 
ABANDONED ONSHCRE OII FIEIDS RITH OVER 250,000 BBL. COMULATIVE ERODUCTION

DST DISC ABD. PID NTM PIELD NAME

$04 \quad 1954 \quad 1973 \quad 51046348$ IA SARA (NO. 30 SAND

DST RISC AD. PIN TOM FIELD NAME

04. $19551973 \quad 17535576$ CHESS (NO. 44)

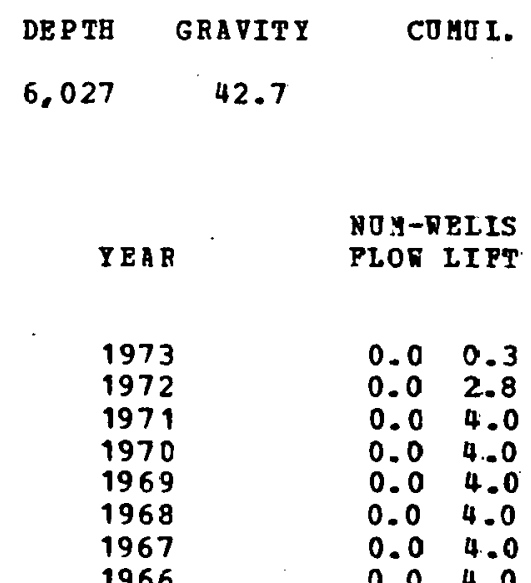

1966

\begin{abstract}
DEPTH GRAVITY
\end{abstract}
6,975

40.3

CONOL. PROD. OF FIELD

580.780

$$
\text { OIL-PRD E/RD }
$$

GAS-PBD

$\begin{array}{rr}316 & 2.6 \\ 4686 & 4.7 \\ 6414 & 4.4 \\ 8157 & .5 .6 \\ 6736 & 4.6 \\ 6909 & 4.7 \\ 7433 & 5.1 \\ 16902 & 11.6\end{array}$

COONTY

DILIACY

$\begin{array}{lrrrrr}\text { YEAR } & \begin{array}{r}\text { NOK-PELLS } \\ \text { FIOW LIFT }\end{array} & \text { OIL-PRD } & \text { E/HD } & \text { GAS-PRD } \\ & & & & & \\ 1973 & 0.0 & 0.1 & 81 & 2.7 & 137 \\ 1972 & 0.0 & 1.0 & 1259 & 3.4 & 4364 \\ 1971 & 0.0 & 1.4 & 1855 & 3.6 & 2767 \\ 1970 & 0.0 & 3.6 & 2219 & 1.7 & 6115 \\ 1969 & 0.0 & 4.0 & 4516 & 3.1 & 13868 \\ 1968 & 0.0 & 4.0 & 5433 & 3.7 & 20200 \\ 1967 & 0.4 & 6.2 & 9544 & 3.9 & 32388 \\ 1966 & 1.0 & 6.5 & 13067 & 4.8 & 30689\end{array}$

COINTY: WILLACY
2 FIELD (S) 
ABA NDONED ONSHORE OIL FIELDS ITH OVER 250,000 BBL. COHOLATIVZ PRODUCTICN

DST DISC ABD. PID NOH PIELD NAME

$04 \quad 19571972 \quad 22128001$ CUELLAR

DST DISC ABD. PLD NUM FIELD NAME

$04 \quad 19341966 \quad 19914001$ COMITAS

DEPTH
1,600

YEA

197
197
197
1969
196
1967
1966
DE РT н
800

\section{GRAVIT Y}
20.6

YEAP

1966

20.9

\begin{abstract}
GRAVITY
\end{abstract}

$$
\begin{aligned}
& \text { NDM-NELIS } \\
& \text { FLON LIFT }
\end{aligned}
$$

$$
\begin{array}{ll}
0.0 & 10.8 \\
0.0 & 13.0 \\
0.0 & 13.0 \\
0.0 & 13.0 \\
0.0 & 13.0 \\
0.0 & 13.0 \\
0.0 & 13.0
\end{array}
$$

COMOI. PROD. OF FIELD

$2,626,525$

NOM-NELIS
FION LIPT

$0.0 \quad 6.7$

$2,818,280$
C.ONTY

2A PATA

$$
\text { OIL-PRD E/WD GAS-PRD }
$$

$\begin{array}{ll}1188 & 0.3 \\ 2537 & 0.5\end{array}$

$3243 \quad 0.7$

$3955 \quad 0.8$

44550.9

$4493 \quad 0.9$

51151.1

COONTY

ZAPATA

OIL-PRD E/RD

GA S-PRD 
A BANDONED ONSHORE OIL FEELDS VITH OVER 250,000 3BL. COMOLATIVE PRODUCTICN

DST NISC ABD. FLD NTM FIELD NAME

$0419211976 \quad 62065009$ MT RANDO VALLEY

\begin{tabular}{|c|c|c|}
\hline DE FTH & GRAVIT Y & CO ID I. \\
\hline 1,900 & 20.9 & \\
\hline YEAR & & $\begin{array}{l}\text { YUM-NELIS } \\
\text { FLOW LIFT }\end{array}$ \\
\hline $\begin{array}{l}1976 \\
1968 \\
1967 \\
1966\end{array}$ & & $\begin{array}{ll}0.0 & 0.2 \\
0.0 & 3 . .5 \\
0.0 & 7.0 \\
0.0 & 7.0\end{array}$ \\
\hline
\end{tabular}

PROD. OF FIELD

$1,739,271$
COUNTY

ZAP ATA

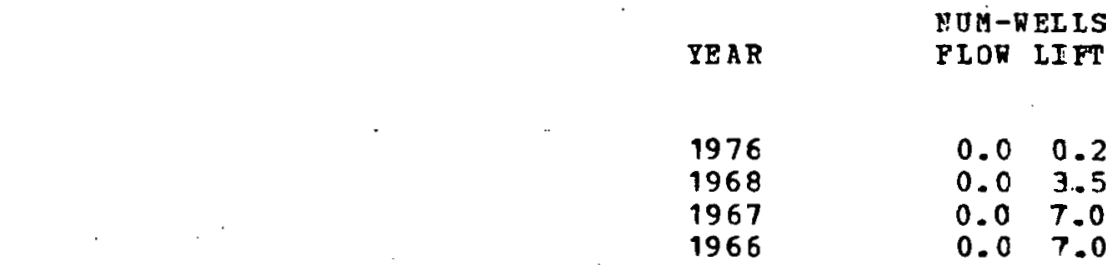

COUNTI: ZAPATA

DISTRICT 04
3 PIELD (S)

172 PIELDS

$\begin{array}{rrr}\text { OIL-PRD } & \text { E/HD } & \text { GAS-PRD } \\ & & \\ 46 & 0.8 & 2 \\ 211 & 0.2 & 3 \\ 3165 & 1.2 & 12 \\ 3523 & 1.4 & 0\end{array}$

$7,184,076$ COMULATIVE PROD. FROM ABANLONEL PIEIDS $240,886,516$ COMOLATIVE PROD. PROM ABANDONED FIELDS 
ABANDONED ONSHORE OIL FIELDS YITH OVER 250,000 BBL- CDUOLATIVE ERODOCTION

DST DISC ABD. FID NOM FIELD NAME

$05 \quad 19551967 \quad 32706333$ PRANRSTON (JAMES IIME, OP.)

\begin{abstract}
DEPTH GRAVITY
10,046

49.0
\end{abstract}

\begin{tabular}{|c|c|c|}
\hline & NOM- & REL IS \\
\hline YEAR & PLON & LIFT \\
\hline $\begin{array}{l}196.7 \\
1966\end{array}$ & $\begin{array}{l}2.3 \\
3.0\end{array}$ & $\begin{array}{l}0.0 \\
0.7\end{array}$ \\
\hline
\end{tabular}

DEPTH GRAVITY

8.850

43.0
COHOL. PROD. OP PIELD

461,019

$\begin{array}{rrr}\text { OIL-PRD } & \text { E/RD } & \text { GAS-PRD } \\ & & \\ 36144 & 42.4 & 149799 \\ 72471 & 54.2 & 312377\end{array}$

COMOL. PROD. OP FIELD

270.075

COONTY

HENDERS ON
COONTY

BEN DERSON

$05 \quad 1965 \quad 1974 \quad 22759500$ DAR (RODESSA G)

IEAR
1974
1973
1972
1971
1970
1969
1968
1967
1965

2 P IEID (S)
CONNTY : HEN DERSON
OIL-PRD E/HD GAS-PRD

$\begin{array}{rrr}966 & 5.3 & 6 \\ 282 & 1.0 & 9 \\ 12674 & 34.7 & 20529 \\ 19647 & 53.8 & 61859 \\ 15877 & 43.5 & 119694 \\ 20266 & 55.5 & 71648 \\ 34492 & 94.5 & 158334 \\ 61528 & 168.6 & 118291 \\ 65688 & 180.0 & 59709\end{array}$

731,094 COMOLATIVE PROD. FROA ABANDONED FIBLDS 
ABAYDONED ONSHORE OIL FIELDS TITH OVER 250,000 BBL. CUHOLATIVE PROEUCTION

DST DTSC ABD. FID NIM FIBLD NASE

of $10441969 \quad 71356001$ PISKTON

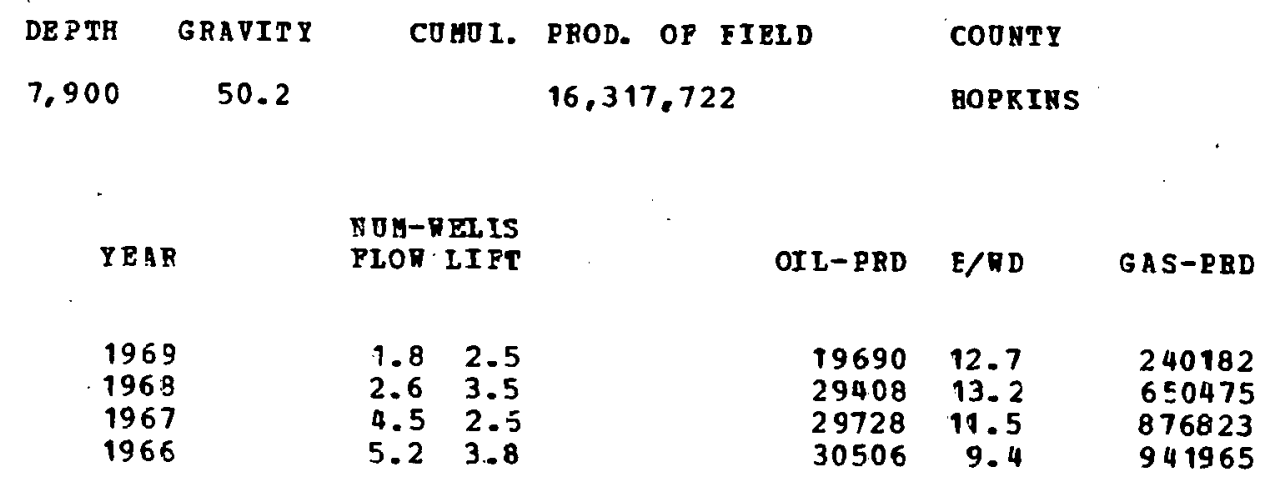

$16,317,722$ CUMOLATIVE PROD. FROE ABAEDONED PIBLDS 
ABANDONED ONSHORE O IL PIELDS RITH OVER 250,000 BBL. COMOLATIVE PRODOCTION

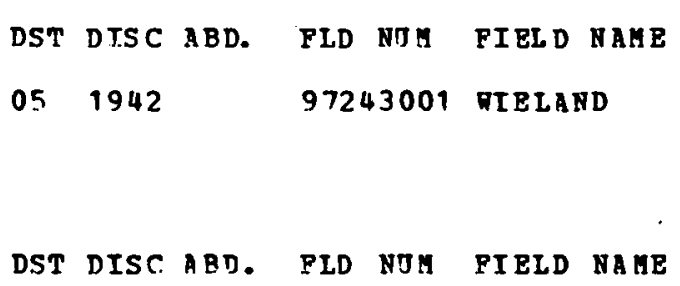

051943195215160001 CAMPBEIL

051943195215160001 CAMPBELL

COONT: HONT
DEPTH GRAVITY COMOL. PROD. OF FIELD COONTY

$\begin{array}{llll}4,352 & 31.0 & 374,690 & \text { HONT }\end{array}$

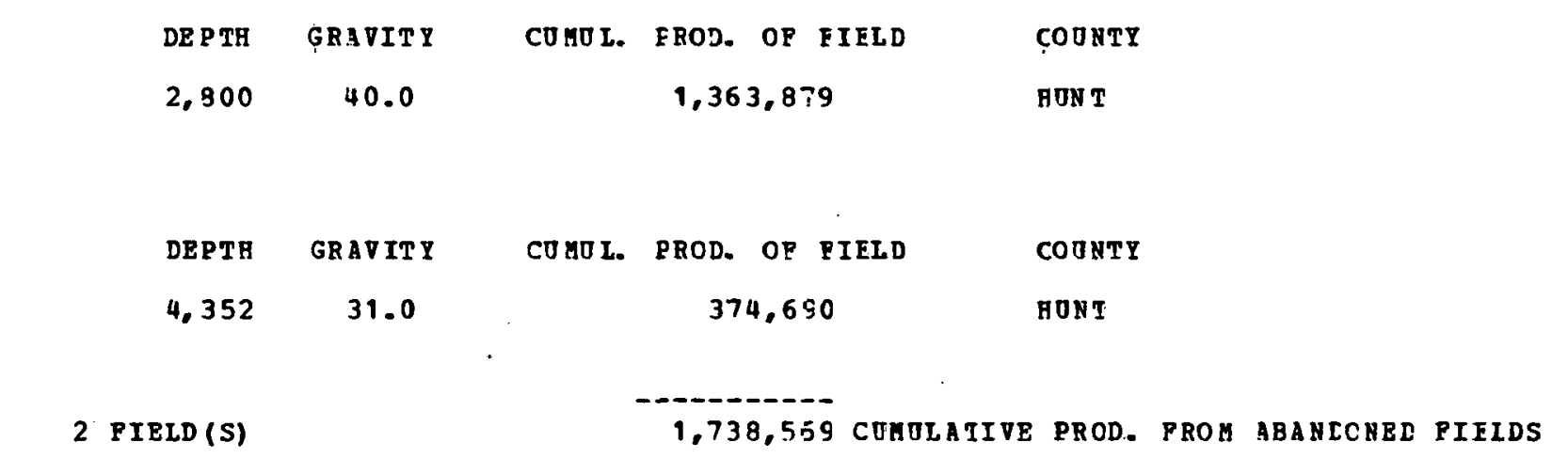


ABA NDONED ONSHORE OIL PIEIDS IITH OVER 250,000 RBL. CUMULATIVE PRODUCTICN

\begin{abstract}
DST DTSC ARD. PLD NTI FIRLD NAHE
051950196994991666 WAITER PAIR (nCODBINE)
\end{abstract}

051951196638399110 HAM GOSSETT (DEXTER, LO.)

\begin{tabular}{|c|c|c|c|c|}
\hline DE $\supseteq T M$ & GRAVITY & СОMOI. & PROD. OF PIELD & $\operatorname{CCONTY}$ \\
\hline 146 & 30.0 & & $. \quad 487,120$ & RAOFMAN \\
\hline
\end{tabular}

YEAR

NOH-WELIS

1969

1968

1968

1966

\begin{abstract}
DEPTH GRAVITY
\end{abstract}
4.030

36.0

\section{YEAR}

1966
FLOW LIFT

$$
\begin{array}{ll}
0.0 & 0.5 \\
0.0 & 1.0 \\
0.0 & 1.0 \\
0.0 & 1.0
\end{array}
$$

COHOL. FROD. OF PIELD

$\operatorname{CONNT}$

258,815

RAIIFHAN

$\begin{array}{rrr}\text { OIL-PRD } & \text { E/RD } & \text { GAS-PRE } \\ & & \\ 1550 & 8.5 & 6 \\ 2683 & 7.4 & 8 \\ 4367 & 12.0 & 12 \\ 5826 & 16.0 & 0\end{array}$

NOH-VELIS

OIL-PRD E/WD

GA S-P BD

$0.0 \quad 0.9$

$1465 \quad 4.4$

0

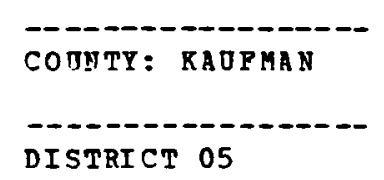

IILDS
745,935 COMOLATIVE PROD. PROM ABANLONED PIELDS $19,533,320$ COMULATIVE PROD. PROM ABANLONEL FIEIDS 
ABA NDONED ONSHORE OIL PIELDS VITH OVER 250,000 BBL. CUMULATIVE PRODUCTICN

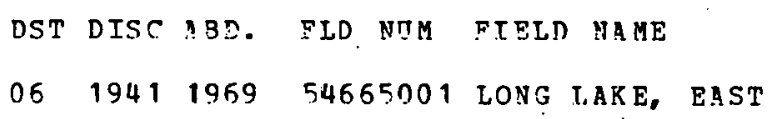

\begin{tabular}{|c|c|c|c|c|c|c|}
\hline \multirow{2}{*}{$\begin{array}{l}\text { DEPTH } \\
5,320\end{array}$} & \multirow{2}{*}{$\begin{array}{c}\text { SRAVITY } \\
42.8\end{array}$} & COMUL. & \multicolumn{2}{|c|}{ PROD. OF PIEID } & \multicolumn{2}{|l|}{ COONTY } \\
\hline & & & $3,801,80$ & & ANDERSOI & \\
\hline YEAF & & $\begin{array}{l}\text { NOM-WELLS } \\
\text { FLOF LIPT }\end{array}$ & & OII-PRD & $E / W D$ & GAS-PRD \\
\hline $\begin{array}{l}1968 \\
1968 \\
196 \\
1966\end{array}$ & & $\begin{array}{rr}0.0 & 8 . .6 \\
0.0 & 12.0 \\
0.0 & 12.0 \\
0.0 & 11.8\end{array}$ & & $\begin{array}{r}257 \\
7768 \\
12040 \\
19013\end{array}$ & $\begin{array}{l}0.1 \\
1.8 \\
2.7 \\
4.4\end{array}$ & $\begin{array}{r}343 \\
7726 \\
10273 \\
24931\end{array}$ \\
\hline DE PTH & GRAVITY & COMOL. & PROD. OF & PIELD & COUNTY & \\
\hline 10,184 & 52.8 & & 929,73 & & AN D ERSO N & \\
\hline YEAE & & $\begin{array}{l}\text { NOM-NELIS } \\
\text { PLOR LIFT }\end{array}$ & & OIL-PRD & $E / R D$ & GAS-PBD \\
\hline $\begin{array}{l}1973 \\
1972 \\
1971 \\
1970 \\
1968 \\
1968 \\
1967 \\
1966\end{array}$ & & $\begin{array}{ll}1.2 & 0.0 \\
3.0 & 0.0 \\
3.8 & 0.0 \\
4.9 & 0.1 \\
5.3 & 1.0 \\
7.1 & 0.9 \\
7.2 & 0.1 \\
7.3 & 0.8\end{array}$ & . & $\begin{array}{r}7258 \\
23322 \\
30441 \\
40126 \\
50066 \\
90351 \\
87342 \\
87243\end{array}$ & $\begin{array}{l}15.9 \\
2.1 .3 \\
21.8 \\
22.0 \\
21.7 \\
30.9 \\
32.6 \\
29.9\end{array}$ & $\begin{array}{r}63339 \\
244982 \\
211304 \\
189712 \\
172657 \\
206222 \\
118182 \\
85513\end{array}$ \\
\hline
\end{tabular}


ARANDONED ONSHORE OIL FIEIDS WITH OVER 250,000 BBL. COMULATIVE PRODOCTICN

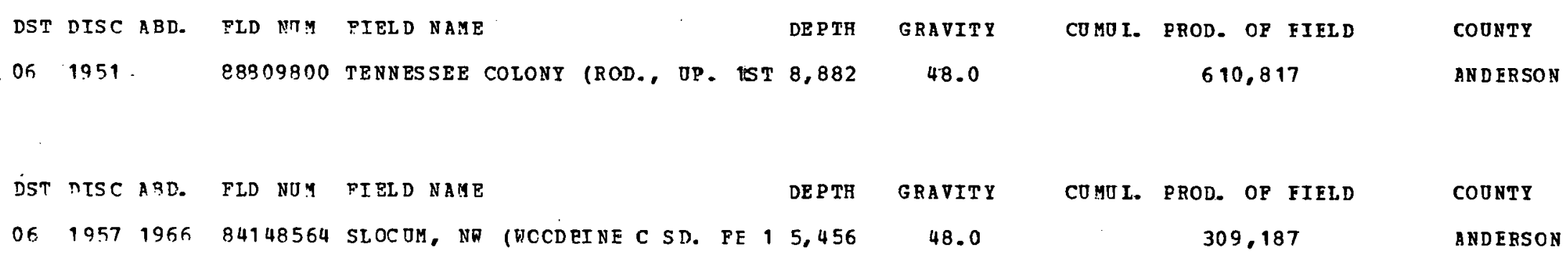

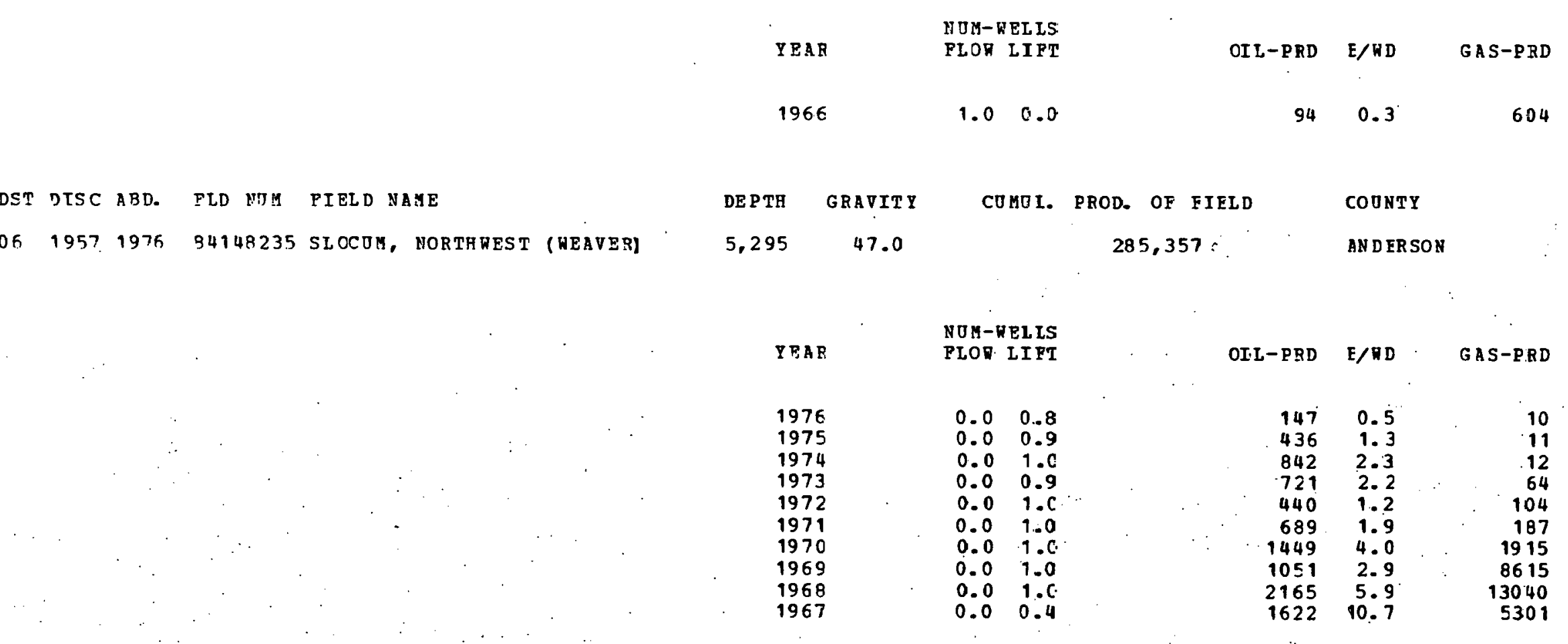


ABANDONED ONSHORE OIL PIELDS NITH OVER 250,000 BBL. CUMULATIVE FROLOCTION

$\begin{array}{ll}\text { DST DISC ABD. FID NOM PIPLD NAME } \\ \text { OE } 1944 & 29824001 \text { BYLAD }\end{array}$

COTNTY: $\mathrm{BONIE}$
DEPTH GRAVITY

7,681

1 PIELD (S)

41.0
CUMUI. EROD. OF FIELD
450,967
COUNTY
EOWIE

$-----1$

450,967 CUMOLATIVE PROD. FROM ABANDONED FIEIDS 
ABANDONED ONSHORE OIL PIELDS ITA OVER 250,000 BBL. COMULATIVE PRODUCTICN

DST DISC ABD. PLD NOM PIELD NAHE

OK $19551973 \quad 77807105$ RODESSA (ANHYCRITZ STR 5750)

$\begin{array}{cc}\text { DEPTH } & \text { GRAVITY } \\ 5,752 & 40.0\end{array}$

5,752

40.0

CUMOL. PROD. OP FIELD

311,353

COONTY

YEAR

1973

197

1970

1969

1968

1967

1966

comnty: cass

1 ?IELD (S)

\begin{tabular}{|c|c|c|c|c|}
\hline $\begin{array}{l}\text { NUA- } \\
\text { FLOW }\end{array}$ & $\begin{array}{l}\text { ELIS } \\
\text { LIFT }\end{array}$ & OIL-PRD & $E / 9 D$ & GAS-PRD \\
\hline $\begin{array}{l}0.0 \\
0.0 \\
0.0 \\
0.7 \\
1.0 \\
1.0 \\
1.0 \\
0.7\end{array}$ & $\begin{array}{l}0.2 \\
1.0 \\
1.0 \\
0.3 \\
0.0 \\
0.0 \\
0.0 \\
0.3\end{array}$ & $\begin{array}{r}48 \\
393 \\
275 \\
1047 \\
1128 \\
1472 \\
1638 \\
1957\end{array}$ & $\begin{array}{l}0.8 \\
1.1 \\
0.8 \\
2.9 \\
3.1 \\
4.0 \\
4.5 \\
5.4\end{array}$ & $\begin{array}{r}1864 \\
23989 \\
60117 \\
65900 \\
71846 \\
78484 \\
96009 \\
109623\end{array}$ \\
\hline
\end{tabular}

31 1, 353 COMOLATIVE PROD. PROM ABREONED FIEIDS 
ARANDONED ONSHORE OTL FIELDS HTH OVER 250,000 BBL. COMULATIVE PRODOCTICN

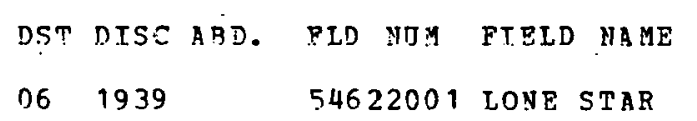

$\begin{array}{cc}\text { DEPTH } & \text { GRAVITY } \\ 4,008 & 37.0\end{array}$

DE PTH

4,846

\section{GRAVITY}

43.0
CUMOL. PROD. JF FIELD

878,051

CUMUL. PROD. OF FIELD

587,165
CCONT

CHEROREE

COU NTY

CHEROKEE.

\begin{tabular}{lll} 
& \multicolumn{2}{l}{ NUM-RELIS } \\
YEAR & FLOR LIFT \\
& & \\
1971 & 0.0 & 2.3 \\
1970 & 0.0 & 3.4 \\
1969 & 0.0 & 4.1 \\
1968 & 0.0 & 4.7 \\
1967 & 0.0 & 5.8 \\
1966 & 0.0 & 6.6
\end{tabular}

\begin{tabular}{|c|c|c|}
\hline OIL-PRD & E/WD & GAS-PRD \\
\hline $\begin{array}{r}1 \\
136 \\
6444 \\
7975 \\
10507 \\
18379\end{array}$ & $\begin{array}{l}0.0 \\
0.1 \\
4.3 \\
4.6 \\
5.0 \\
7.6\end{array}$ & $\begin{array}{r}0 \\
13 \\
117 \\
175 \\
224 \\
2325\end{array}$ \\
\hline
\end{tabular}

COUNTY: CHEROKEE

2 F IELD (S)

$1,465,216$ CUMULATIVE PROD. PROM ABANDONED FIELDS 
ARANDONED ONSHORE OIL PIELDS VITH OVER 250,000 BBL. COUULATIVE PRODUCTICN

DST DISC 9D. PID NOM FIPLD NAME

$05 \quad 1952$
95369016 MASKOM (AKIN SAND)

COOYTY: RARRISON

$\begin{array}{cccc}\text { JEPTH } & \text { GRAVITY } & \text { COHDL. PROD. OF FIELD } & \text { CCONTY } \\ \overline{5}, 198 & 40.0 & 732,986 & \text { EAREISON }\end{array}$

1 PIE D (S)
732,986 COMOLATIVE PROD. PRON ABANCONED PIELDS 
ABANDONED ONSHORE OIL FIELDS WITH OVER 250,000 BBL. COHOLATIVE PRODOCTION

DST DISC ABD. FLD NTI FIELD NAME

$06 \quad 1936 \quad 1970 \quad 36430001$ GRAPELAND

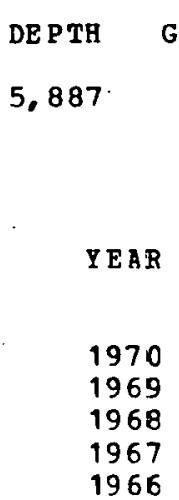

1 FIELD (S)

967

\section{GRAVIT Y}

39.7

NUM- QELIS
FLOW LIPT

$0.0 \quad 5.0$

$\begin{array}{ll}0.0 & 5.0 \\ 0.0 & 7.0\end{array}$

0.07 .0

$0.6 \quad 6.4$

COONTY: HOUSTON

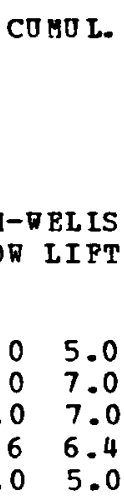

PRCD. OF FIELD

2.401 .448

COUNTY

月O OSTON 
ABANDONED ONSHORE OII. PIEZDS WITH OVER 250,000 BBL. CUMULATIVE PRODUCTICN

DST DISC AED. PID NOM FIELD NAME

$06 \quad 1943$

40353284 HENDERSON (ROSR DO.)

DST DISC ABD. FID. NOM PIELD NAME

Of $19551970 \quad 46793500$ JOHN C. ROBBINS (PETTIT)

DST DISC ABD. FLD NOM FIELD NAME

$06 \quad 1959197566461333$ OAR HILL. (PETTIT)

\begin{tabular}{|c|c|c|c|c|}
\hline DEPTR & GRAVITY & COTHE L. & ROD. OP PIELD & COUNTY \\
\hline 7.377 & 42.7 & & $3,692,020$ & EOSK \\
\hline
\end{tabular}

\begin{tabular}{|c|c|c|c|c|}
\hline DE PTH & GRAVITI & COMTL. & PROD. OF PIELD & CCONTY \\
\hline & 43.0 & & 566,295 & ROSK \\
\hline
\end{tabular}

YEAR
1970
1969
1968
1967
1966

\section{NOM-TELIS
PLON IIPT}

CIL-PRD E/WD.

GAS-P RD

$$
\begin{array}{ll}
0.0 & 0.9 \\
0.0 & 1.0 \\
0.0 & 4.8 \\
0.0 & 6.0 \\
0.0 & 5.2
\end{array}
$$

$\begin{array}{rr}321 & 1.0 \\ 11.53 & 3.2 \\ 1314 & 0.8 \\ 1536 & 0.7\end{array}$

6851
24675

20738

25061

25061
51785

DEPTH

GRAVITY

cono

\begin{tabular}{|c|c|c|}
\hline & \multicolumn{2}{|c|}{ NOM-RELLS } \\
\hline YE AR & PLOR. & $L=\mathrm{FI}$ \\
\hline $\begin{array}{l}1975 \\
1974 \\
1973 \\
1972 \\
1971 \\
1970 \\
1969 \\
1968 \\
1967 \\
1966\end{array}$ & $\begin{array}{l}0.0 \\
0.0 \\
0.0 \\
0.0 \\
0.3 \\
0.1 \\
0.0 \\
0.0 \\
0.0 \\
0.0\end{array}$ & $\begin{array}{l}0.04 \\
1.0 \\
1.0 \\
1.0 \\
0.0 \\
3.9 \\
1.0 \\
1.0 \\
1.0 \\
1.0\end{array}$ \\
\hline
\end{tabular}

7,165

40.0
GA S-P RD

306

1812

5001

8875

19752

22039

6050

4555

754 
ABANDONED ONSHORE OII FIELDS IITH OVER 250,000 BBL. CUGULATIVE FRODOCTION

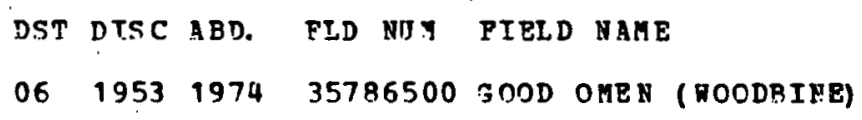

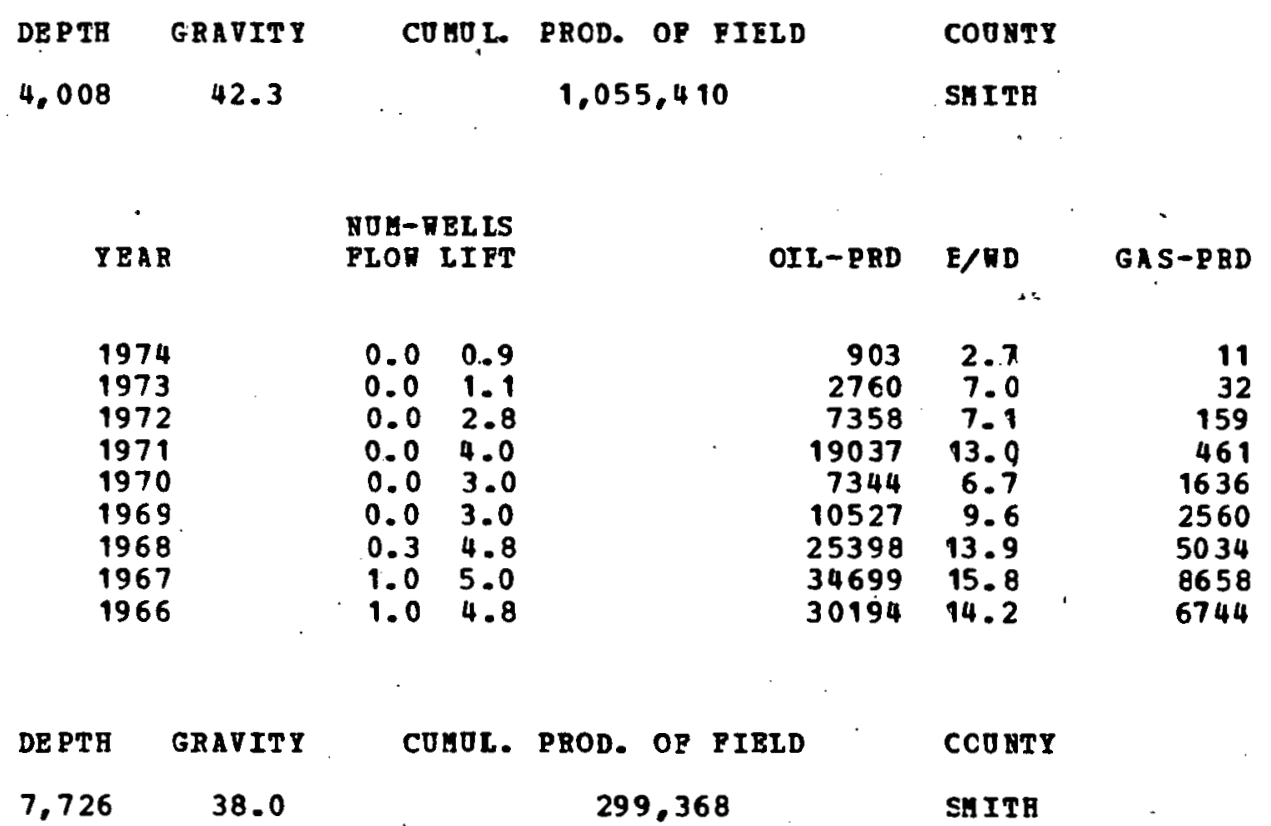

2 P IE LD (S) 
ABANDONED ONSHOFE OIL FIELDS WITH OVER 250,000 BBL. CCMULATIVE PRODUCTION

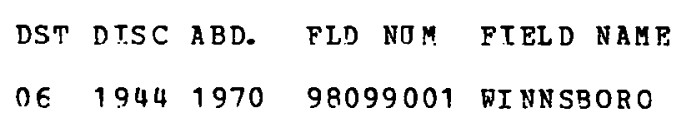
DE P TH
GRAVIT $\mathbf{Y}$
COHO L. PROD. OP FIELD
COUNTY
8,260
42.0
$1,191,549$
nOO L

\begin{tabular}{|c|c|}
\hline \multicolumn{2}{|c|}{ NOH-VELIS } \\
\hline FION & L I $? \mathrm{~T}$ \\
\hline $\begin{array}{l}1.0 \\
1.0 \\
1.0 \\
1.2 \\
2.0\end{array}$ & $\begin{array}{l}0.0 \\
0.2 \\
1.0 \\
1.8 \\
1.0\end{array}$ \\
\hline
\end{tabular}

$\begin{array}{rrr}\text { OIL-PRD } & \text { E/HD } & \text { GAS-PBD } \\ & & \\ 423 & 1.2 & 540 \\ 717 & 1.7 & 1641 \\ 951 & 1.3 & 4218 \\ 2745 & 2.5 & 36492 \\ 7331 & 6.7 & 140886\end{array}$
DE PTH
GRAVITY

8,360

6.3.0

CUMUE. PROD. OF PIELD

COUNTY

921,610

nOO C

\begin{tabular}{|c|c|c|}
\hline \multirow{2}{*}{ YEAR } & \multicolumn{2}{|c|}{ NOH-HELIS } \\
\hline & FLON & LI PT \\
\hline$-197 \epsilon$ & 0.0 & 0.3 \\
\hline 1975 & 0.0 & 1.0 \\
\hline 1974 & 0.0 & 1.0 \\
\hline 1973 & 0.0 & 1.5 \\
\hline 1972 & 1.9 & 2.0 \\
\hline 1971 & 2.9 & 1.5 \\
\hline 1970 & 3.0 & 0.0 \\
\hline 1969 & 3.0 & 0.3 \\
\hline 1968 & $3 . .0$ & 1.. 0 \\
\hline $\begin{array}{l}1967 \\
1966\end{array}$ & $\begin{array}{l}3.7 \\
5.0\end{array}$ & $\begin{array}{l}1.0 \\
1.0\end{array}$ \\
\hline
\end{tabular}


43A NDONED ONSHORE OIL FIELDS ITT OVER 250,000 BBL. CONOLATIVE PRODOCTICH

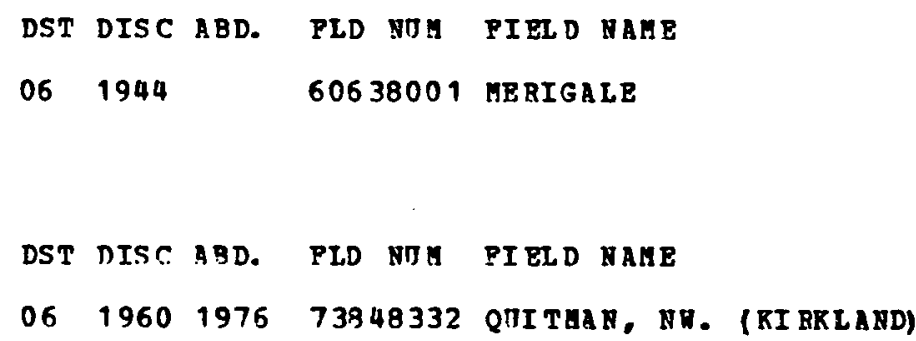

\begin{tabular}{|c|c|c|c|}
\hline DE PTH & GRAVITY & \multicolumn{2}{|c|}{ CU HO L. } \\
\hline 5,100 & 25.0 & & \\
\hline DE PTH & GRAVITI & \multicolumn{2}{|c|}{ CO סִ I. } \\
\hline 8,356 & 42.0 & & \\
\hline YEAE & & \multicolumn{2}{|c|}{$\begin{array}{l}\text { NOM-RELLS } \\
\text { PLOR LIPT }\end{array}$} \\
\hline $\begin{array}{l}1976 \\
1975 \\
1972 \\
1971 \\
1970 \\
1969 \\
1968 \\
1967 \\
1966\end{array}$ & & $\begin{array}{l}0.0 \\
0.0 \\
0.3 \\
1.4 \\
2.3 \\
3.6 \\
5.0 \\
6.8 \\
8.9\end{array}$ & $\begin{array}{l}0.3 \\
0.9 \\
0.0 \\
0.0 \\
0.0 \\
0.0 \\
0.0 \\
1.0 \\
2.2\end{array}$ \\
\hline
\end{tabular}

DE PTR

GRAVITY

5,550

24.0

CUMOL. PROD. OP FIELD

484,632

COUNTY

noo $\mathrm{C}$

\begin{tabular}{lll} 
& \multicolumn{2}{c}{ NOH-YELIS } \\
YEAR & PLOR LIPT \\
& & \\
1976 & 0.0 & 0.3 \\
1975 & 0.0 & 1.0 \\
1974 & 0.0 & 0.9 \\
1972 & 0.0 & 0.9 \\
1970 & 0.0 & 1.0 \\
1969 & 0.0 & 1.0 \\
1968 & 0.0 & 1.0 \\
1967 & 0.0 & 1.0 \\
1966 & 0.0 & 1.0
\end{tabular}

COONTY

noo $\mathrm{c}$

COONTY

RCOL

E/WD GAS-PRD

$\begin{array}{rr}5.3 & 3 \\ 4.9 & 11 \\ 6.8 & 19874 \\ 4.5 & 63454 \\ 2.6 & 57949 \\ 4.1 & 103623 \\ 5.4 & 104135 \\ 4.1 & 157193 \\ 6.1 & 231672\end{array}$

3

9874

63454

$\begin{array}{llr}2189 & 2.6 & 5799 \\ 5416 & 4.1 & 103623\end{array}$

$\begin{array}{lll}9776 & 5.4 & 104135\end{array}$

$\begin{array}{lll}11535 & 4.1 & 157193 \\ 24532 & 6.1 & 231672\end{array}$

$\begin{array}{rrr}\text { OIL-PRD } & \text { E/RI } & \text { GAS-PRD } \\ & & \\ 681 & 7.5 & 3 \\ 5467 & 15.0 & 12 \\ 4239 & 12.7 & 11 \\ 725 & 2.2 & 2 \\ 228 & 0.6 & 1 \\ 3235 & 8.9 & 129 \\ 4044 & 11.1 & 1025 \\ 6984 & 19.1 & 1669 \\ 7757 & 21.3 & 1912\end{array}$


ABANDONED ONSHOBE OIL PIELDS ITH OVER 250,000 BBL. CE GULAT IVE PRODUCTION

DST DISC ABD. PLD NOM FIEID NAME

061959197273845250 QTITHAN, BAST (RIRRLAND)

DST RISC ABD. FLD NOM PIELD NAGE

$061961 \quad 197473879400$ OUITHAN GLADYS (HENDERSON)

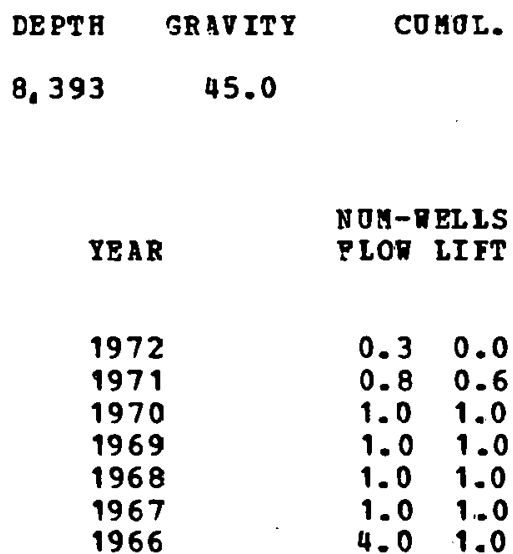

DEPTH GRAVIT Y

8,269

45.0

COHO 1. PROD. OF FI EID

\begin{tabular}{lll} 
& \multicolumn{2}{c}{ NOK-RELIS } \\
YEAR & PLOR LIFT \\
& \multicolumn{3}{c}{} \\
1974 & 0.0 & 0.4 \\
1973 & 0.1 & 1.0 \\
1972 & 1.8 & 0.3 \\
1971 & 2.0 & 0.0 \\
1970 & 2.0 & 0.0 \\
1969 & 2.6 & 0.0 \\
1968 & 3.0 & 0.0 \\
1967 & 3.0 & 0.0 \\
1966 & 2.5 & 0.5
\end{tabular}

COURT

309.182

nCOE

342.632

$\operatorname{CONTY}$

nood

$\begin{array}{rrr}\text { OIL-PRD } & \text { E/RE } & \text { GAS-PRD } \\ & & \\ 31 & 0.3 & 4497 \\ 1780 & 3.4 & 70945 \\ 3206 & 4.4 & 54236 \\ 3750 & 5.1 & 78549 \\ 6061 & 8.3 & 83799 \\ 6747 & 9.2 & 74812 \\ 10689 & 5.9 & 91838\end{array}$

$\begin{array}{rrr}\text { OIL-PRD } & \text { E/NI } & \text { GAS-PRD } \\ & & \\ 111 & 0.7 & 1702 \\ 742 & 1.9 & 9866 \\ 3357 & 4.6 & 38792 \\ 2875 & 3.9 & 47154 \\ 5367 & 7.4 & 127288 \\ 8697 & 9.2 & 181095 \\ 19540 & 17.8 & 312334 \\ 36723 & 33.5 & 375799 \\ 15781 & 14.4 & 91043\end{array}$


ABANDONED ONSHORE OIL PIELDS RITH OVER 250,000 B8L. COMULATIVE PRODOCTICN

\begin{abstract}
DST DISC A3D. FLD NIM FIELD NAME
$051951 \quad 1975 \quad 24404333$ DEM PREE (SUB-CLARKSUILLE)
\end{abstract}

$\begin{array}{cc}\text { DE PTH } & \text { GRAVITY } \\ 4,990 & 28.5\end{array}$

COMO1. PROD. OF PIELD

259.486

COUNTY

noO C

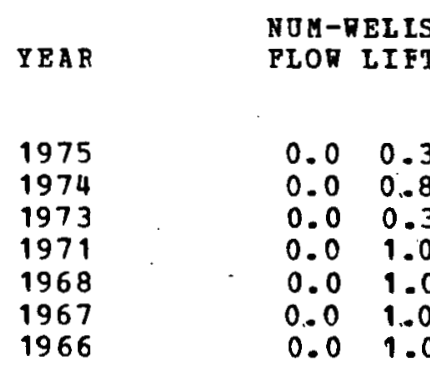

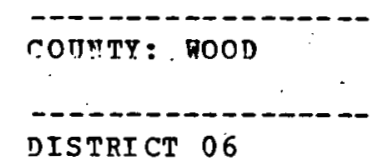

DISTRICT 06
8 FIELD (S)

24 FIEIDS

$\begin{array}{rrr}\text { OIL-PRD } & \text { E } R D & \text { GAS-PRD } \\ & & \\ 662 & 7.3 & 3 \\ 1529 & 5.0 & 12 \\ 596 & 4.9 & 4 \\ 2279 & 6.2 & 9 \\ 1882 & 5.2 & 11 \\ 2658 & 7.3 & 54 \\ 2421 & 6.6 & 48\end{array}$

$4,945,142$ CUMOLATIVE PROD. PROA ABANDONEC PIRLDS $22,188,152$ CUMOLATIVE PROD. FROM ABANDONED RIEIDS 
ABANDONED DNSHORE OEL FIELDS WITH OVER 250,000 BBL. COMOLATIVE FRODUCTION

IIS! DISC ABD. PLD NOM PIRLD NAME

O?R. 1954196140730666 HERRTRING (MORAN)

DST DTSC BD. FID NOY FIELD NAME

$07 R 1950197363026333$ MORRISETT (TANNEHILI, LO.)

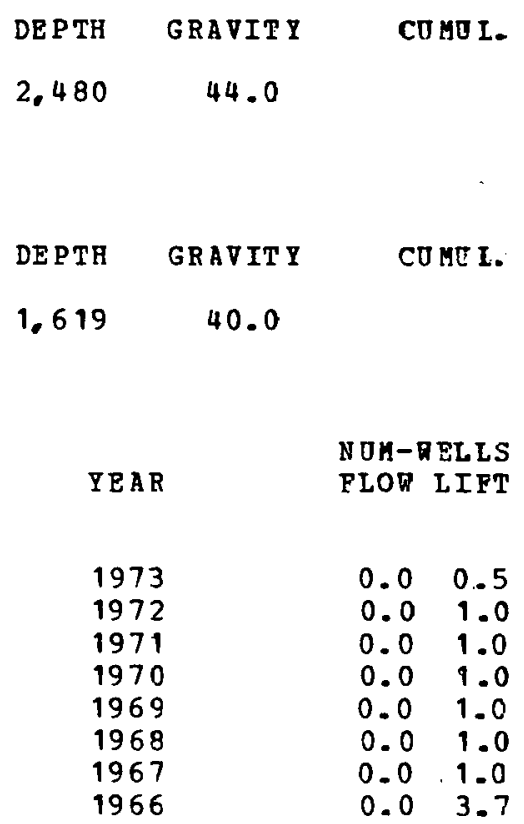

DE PTH

1.506
41.0
COMOL. PROD. OF FIELD

352,849
COONTY

CALLAHAN .

CODNTI

CALIAHAN
DST DISC ABD. FLD NOM FIELD NAME

OTR $19551968 \quad 64150500$ MYERS (HOPE, OPPER)

\section{OIL-PFD E/WD GAS-PRD}

$\begin{array}{rl}12 & 0.1 \\ 46 & 0.1 \\ 103 & 0.3 \\ 265 & 0.7 \\ 144 & 0.4 \\ 189 & 0.5 \\ 203 & 0.6 \\ 1153 & 0.9\end{array}$

C:ONTY

CALLAHAN

\begin{tabular}{|c|c|c|c|c|}
\hline & NUB-RELIS & & & \\
\hline YEAR & PLOW IIFT & OIL-PRD & $E /$ ตD & GAS-PRD \\
\hline $\begin{array}{l}1968 \\
1967 \\
1966\end{array}$ & $\begin{array}{ll}0.0 & 12.5 \\
0.0 & 15.0 \\
0.0 & 13.7\end{array}$ & $\begin{array}{r}1264 \\
10950 \\
15039\end{array}$ & $\begin{array}{l}1.3 \\
2.0 \\
3.0\end{array}$ & $\begin{array}{r}30 \\
54 \\
0\end{array}$ \\
\hline
\end{tabular}


ABANDONED ONSHORE OIL FIELDS MITH OVER 250,000 BBL. CUMOLATIVE FRODOCTION

DST DISC ABD. PID NOM FIELD NAGE

O7R 1953197079706500 ST. PATRICR (HOPE)

DST DISC ABD. PID NOE TIELD HAHE

079 19541969347.19500 GIDDENS (1900 SA ND)

$\begin{array}{lcc:c}\text { DEPTH } & \text { GRAPITY } & \text { COHOL. PROD. OP FIELD } & \text { CODNTY } \\ 1.772 & 42.0 & 292.406 & \text { CALIAHAN }\end{array}$

YEAR

ROH-RELIS

PLON LIFT

1970

1969

1968

1967

1966

DEPTH GRAVITY

1,920

40.4

OIL - PRD

E/最 C

$\begin{array}{rr}21 & 0.0 \\ 4479 & 1.1 \\ 4476 & 1.1 \\ 6278 & 1.6 \\ 12031 & 3.0\end{array}$

0. 0

1. 1

1.6
3.0

0.010 .8

COYUL. PROD. OP FIELD

260,433

COONTY

CALIAHAY

\section{NOH-RELLS}

TEAR FLOR IIFT

1963

1963

1967

1966

5 FIELD (S)

(s)

$\begin{array}{rr}\text { CIL-PRD } & \text { E/R } ~ \\ & \\ 1329 & 0.5 \\ 12375 & 2.0 \\ 25900 & 3.6 \\ 24843 & 3.1\end{array}$

24843 3.
A-PRD

1012

3636

3296

2788
GA S-PRD

88
643
2297
0


ARANDONED ONSHORE OIL FEEIDS WITH OVER 250,000 BBL. COMOLATIVR PRODUCTICN

DST DISC ABD. PLD NTM PTELD YAME

O7B $19421976 \quad 19395001$ COKER

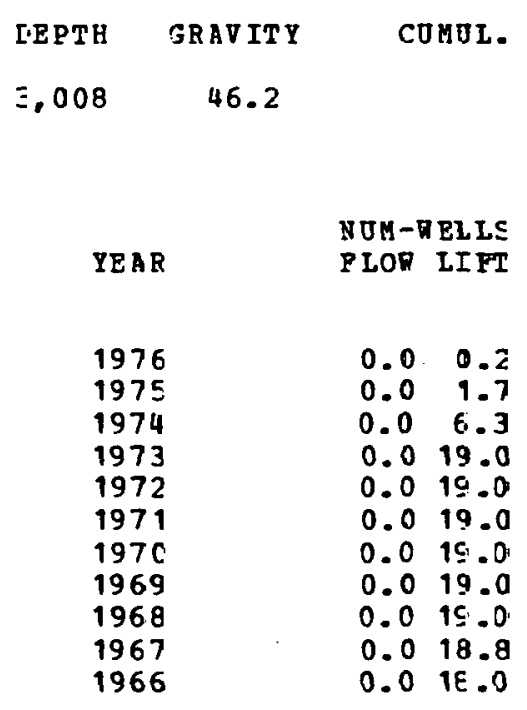

LEPTE GRAVITY

3. 475
COHEI. PROD. OP FIELD

307.939
COONTY

$1,705,019$

COL EMAN
DST DISC ABD. PID NOM FIELD NAME

O7B 1950197560026250 MC RILLIAMS-DAVIS (FAY)
40.0

\section{NOM-VELLS}

YEAR FION II FT

1975
1974
1973
1972
1971
1970
1969
1968
1967
1966

$$
\begin{array}{ll}
0.0 & 0.3 \\
0.0 & 1.7 \\
0.0 & 11.7 \\
0.0 & 2.0 \\
0.0 & 2.0 \\
0.0 & 4.5 \\
0.0 & 4.8 \\
0.0 & 5.0 \\
0.0 & 5.0 \\
0.0 & 5.0
\end{array}
$$

1966 $\equiv / R D$

GA S-P RD

$\begin{array}{rr}13 & 0.2 \\ 2482 & 4.1 \\ 2697 & 1.2 \\ 4060 & 0.6 \\ 10082 & 1.5 \\ 13900 & 2.0 \\ 10268 & 1.5 \\ 30014 & 4.3 \\ 16108 & 2.3 \\ 14804 & 2.2 \\ 8707 & 1.3\end{array}$

1
10
800
1800
1800
1800
1800
1960
2506
7969
25383

COJNTI

COLEGAN

$\begin{array}{rr}\text { OIL-PBD } & \text { E/HD } \\ & \\ 50 & 0.4 \\ 362 & 0.6 \\ 409 & 0.7 \\ 530 & 0.7 \\ 694 & 1.0 \\ 1125 & 0.7 \\ 2251 & 1.3 \\ 2954 & 1.6 \\ 3334 & 1.8 \\ 3674 & 2.0\end{array}$

GA S-PRD

7
80
36
80
217
370
1083
1876
3225
0


ABANDONED ONSHORE OIL FIEIDS WITH OVER 250,000 BBL. CUMULATIVE PRODUCTION

DST DISC ABD. FID NOM FIELD NAME

07 B 19501960 88751875 TEMPLETON (JENMI NGS SAND)
DEPTH

3,350

FIELD (S)
GRAV ITY

47.0
COONTY: COLEMAN
CUMOL. PROD. OP FIELD

271,647

CON NTY

COLEMAN
$2,284,605$ CDMOLAIIVE PROD. PROB ABANLONEL PIELDS 
ABANDONED ONSHORE OIE FIELDS WITH OVER 250,000 BBL. COMOLATIVE PBODOCTION

DST DISC ABD. FLD NOM EIFLD NAME

O7D $19541973 \quad 74863200$ PAVEN CREEK (CANYON SAND)

DST DISC ARD. FLD NTM FIELD NAME

07 P 1955197274863400 PAVEN CREEK (NOODLE CREEK)

DEPTH
4,228

YEAR

1973
1972
1971
1970
1969
1968
1967
1966

DEPTH

2.,657

GRAVIT 7

40.2

42.5
COMOL. PROD. OP FIELD

$1,347.477$

CONTY

NOM-WELIS
FLON LIPT

$0.0 \quad 2.6$

0.0 .4 .9

0.08 .3

$0.0 \quad 13.6$

0.019 .0

0.228 .7

$\begin{array}{ll}0.0 & 24.4 \\ 0.0 & 23.4\end{array}$

COMO L. PROD. OF FIELD

920,007

$\begin{array}{rrr}\text { OIL-PRD } & \text { E/RD } & \text { GAS-P } 3 D \\ & & \\ 1068 & 1.1 & 7313 \\ 3171 & 1.8 & 9648 \\ 6381 & 2.1 & 10780 \\ 15995 & 3.2 & 17724 \\ 31436 & 4.5 & 27169 \\ 50074 & 4.7 & 59297 \\ 31574 & 3.5 & 49071 \\ 21901 & 2.6 & 77017\end{array}$

CכONTY

PISHER

YEAP
1972
1971
1970
1960
1968
1967
1966

NOM-RELLS

FLOW LIFT

OIL-PRD E/WD

GA S-P EDD

1972

1971
1970

1969

$\begin{array}{ll}0.0 & 2.7 \\ 0.0 & 5.5 \\ 0.0 & 6.4 \\ 0.0 & 6.2 \\ 0.0 & 8.0 \\ 0.0 & 8.0 \\ 0.0 & 8.0\end{array}$

$\begin{array}{rr}363 & 0.4 \\ 1129 & 0.6 \\ 2115 & 0.9 \\ 2351 & 1.0 \\ 5049 & 1.7 \\ 5449 & 1.9 \\ 7306 & 2.5\end{array}$

10
36
54
869
158
331
391

10
36
54
869
2158
2331
2391


ABA NDONED OHSBORE OIL FIELDS ITH OVER 250,000 BBL. COHULATIVE PRODUCTICN

DST DISC ABD. FID NTI PIELD NAHE

O7R 1955197377491500 RO9Y, YORTH (CISC)

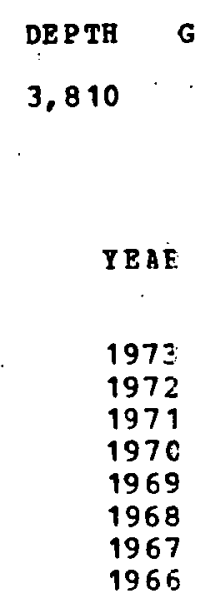

DE P TA

3,810

TEAE

1973

1972

1971

1970

1969

1968

1967

1966

COUNTY PISHER
3 FIELD (S)
COONTY

FIS BER

$\begin{array}{rrrrr}\begin{array}{l}\text { MOH-RELIS } \\ \text { PLOR LIPT }\end{array} & \text { OIL-PRD } & \text { E/RD } & \text { GAS-PRD } \\ & & & & \\ 0.0 & 0.7 & 1168 & 4.8 & 11 \\ 0.0 & 1.8 & 2707 & 4.0 & 21 \\ 0.0 & 2.0 & 4185 & 5.7 & 276 \\ 0.0 & 2.0 & 4719 & 6.5 & 442 \\ 0.0 & 2.0 & 3946 & 5.4 & 296 \\ 0.0 & 2.0 & 3408 & 4.7 & 962 \\ 0.0 & 2.0 & 6792 & 9.3 & 1390 \\ 0.0 & 2.0 & 10787 & 14.8 & 0\end{array}$

2,648, 150 CUMOLATIVE PROD. PROM ABANCONEC FIELDS 
ABA NDONED ONSHJRE OII FIEIDS NTT OVER 250,000 BBL. COHULATIVE PRODOCTICN

DST DISC ABD. PLD NTM FIELD YAME O7B $1941 \quad 1976 \quad 52496001$ LAYSONON

DST NISC APD. FLD NOM FIELD NAME

07R 1958197196003333 WEINERT, SOJTR (ZONE A)

DEPTH
2,684
YEAB

1976
1975
1974
1969
1968
1967
1966

\section{RAV IT Y}

40.0

COMJL. PROD. OF FIELD

$1,175,877$

$\operatorname{CCONTY}$

EAS REL

NOM-RELLS

PLOW LEFT

OIL-PRD E/RD

GA S-PRD

$\begin{array}{ll}0.0 & 0.1 \\ 0.0 & 1.0 \\ 0.1 & 0.3 \\ 0.0 & 1.4 \\ 0.0 & 2.7 \\ 0.0 & 1.0 \\ 0.0 & 1.0\end{array}$

$\begin{array}{rr}95 & 3.1 \\ 1354 & 3.7 \\ 8553 & 25.6 \\ 259 & 0.5 \\ 1582 & 1.6 \\ 2247 & 6.2 \\ 2661 & 7.3\end{array}$

9
219
1331
2
12
12
0

DEPTH GRAVITY

COMUL. FROD. OF FIELD

COONTY

4.735

39.0

$1,004,625$

HAS REL

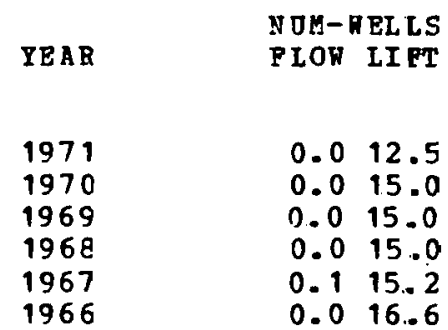

\begin{tabular}{|c|c|c|c|}
\hline LOW IIPT & OIL-PRD & $E / R D$ & GAS-PBD \\
\hline $\begin{array}{ll}0.0 & 12.5 \\
0.0 & 15.0 \\
0.0 & 15.0 \\
0.0 & 15.0 \\
0.1 & 15.0 \\
0.0 & 16.0\end{array}$ & $\begin{array}{l}26343 \\
35161 \\
34904 \\
30469 \\
37994 \\
60322\end{array}$ & $\begin{array}{r}5.8 \\
6.4 \\
6.4 \\
5.6 \\
6.8 \\
10.0\end{array}$ & $\begin{array}{r}8266 \\
8718 \\
8211 \\
6922 \\
7517 \\
14002\end{array}$ \\
\hline
\end{tabular}

GAS-PBD

8266

8718

8211

6922

7517

14002 
ABANDONED ONSHORE OIL FIELDS WITH OVER 250,000 BBL. CUUOLAT IVE PROLUCT ICN

DST DISC ABD. FLD NOM FIELD NAME

O 7B 1958197196003666 DEINERT, SO OTH (ZONE B)

$\begin{array}{cc}\text { DEPTE } & \text { GFAVITI } \\ 4,777 & 39.0\end{array}$

4,777

COUNTY: HASKPL
3 PIELD (S)

\begin{tabular}{|c|c|c|c|c|}
\hline YEAR & $\begin{array}{l}\text { YOH-BELLS } \\
\text { FLOR LIFT }\end{array}$ & OIL - PRD & E/R C & GAS-PRD \\
\hline $\begin{array}{l}1971 \\
1970 \\
1969 \\
1968 \\
1967 \\
1966\end{array}$ & $\begin{array}{ll}0.0 & 11.0 \\
0.0 & 13.0 \\
0.0 & 13.5 \\
0.0 & 15.0 \\
0.0 & 15.0 \\
0.0 & 15.0\end{array}$ & $\begin{array}{l}10642 \\
16695 \\
20121 \\
26262 \\
33941 \\
44651\end{array}$ & $\begin{array}{l}2.7 \\
3.5 \\
4.1 \\
4.8 \\
6.2 \\
8.2\end{array}$ & $\begin{array}{r}3243 \\
3035 \\
3758 \\
5545 \\
6855 \\
14448\end{array}$ \\
\hline
\end{tabular}
COHUL. PROD. OF FIELD
$\operatorname{CONNTY}$
803,881
HASREL

$2,985,383$ COROLATIVE PRCD. PROM ABANCONET FIELDS 
ABANDONED ONSHORZ OII FIELDS NTT OVER 250,000 BBL. CUHOLATIVE PRODUCTICN

\begin{abstract}
DST DISC ABD. PLD VITH PIELD VAME
O7B 1941197265824001 NOODLE, SOJTH
\end{abstract}

DST DISC ABD. PLD NOM PIELD NAME

$078 \quad 1938 \quad 1965 \quad 947001$ AKARD

DST תTSC ARD. - FLD NIM PIELD NAME

$07 R \quad 1951 \quad 1974 \quad 2420500$ AMYSEARS (KING)

$\begin{array}{ccccc}\text { DEPTH } & \text { GRAVITY } & \text { COLOL. } & \text { PROD. OF PIELD } & \text { CONNTY } \\ 3.036 & 44.0 & : & 1.414 .767 & \text { JONES }\end{array}$

YEAR

NOT-RELL.S

OIL-PRD E/RD GAS-PRE

1972

1971

1970

1969

1968

1967

1966

PLON LIFT

$\begin{array}{ll}0.0 & 1.3 \\ 0.0 & 3.0\end{array}$

0.03 .0

0.02 .5

$0.0 \quad 2.6$

0.03 .0

0.03 .0

185

0.4

$\begin{array}{rr}185 & 0.4 \\ 924 & 0.8 \\ 1836 & 1.7\end{array}$

$1836 \quad 1.7$

$\begin{array}{ll}1854 & 2.0 \\ 2840 & 3.0\end{array}$

$\begin{array}{ll}2840 & 3.0 \\ 7297 & 6.7\end{array}$

7297
10874

9.9

4

4
12
12
303
1396
2676
4318

DEPTE GRAVITY

CU UO L. PROD. OP FIELD

COUNT

2,154

39.4

$1,176,333$

JORES

DEPTH GRAVITY

COHOL. PROD. OF PIELD

CCUETI

3,086

44.0

$1,147,532$

JONES
MOM-RELLS FLOR LIFT

$\begin{array}{lll}0.0 & 1.3\end{array}$

0.02 .0

0.02 .0

0.02 .0

0.02 .6

0.03 .7

$0.0 \quad 4.0$

0.04 .0

0.04 .4

$\begin{array}{rr}\text { OIL - PRD } & \text { E/RD } \\ & \\ 493 & 1.0 \\ 1216 & 1.7 \\ 2365 & 3.2 \\ 2039 & 2.8 \\ 996 & 1.1 \\ 1339 & 1.0 \\ 1618 & 1.1 \\ 2012 & 1.4 \\ 3076 & 1.9\end{array}$

GA S-PRD

8
12
12
12
12
12
12
100
2119


APANDONED ONSHORE OII PIEIDS PITH OVER 250,000 BBL. COHULATIVE PRODUCTICH

\begin{abstract}
DST DISC ABD. FLD NDY FIBLD NABE
$07019491964 \quad 43572001$ HTM DHRSY GRAY

DST กIS : 9. FLD NIT PIELD NAME

$07019401976 \quad 30272001$ FARNSEORTH
\end{abstract}

\begin{tabular}{|c|c|c|c|}
\hline DE P IR & GRAVITY & \multicolumn{2}{|c|}{ co au I. } \\
\hline 2,122 & 38.0 & & \\
\hline DE PTH & GRAVITY & \multicolumn{2}{|c|}{ CD MO I. } \\
\hline 2,836 & 40.0 & & \\
\hline Y BAF & & $\begin{array}{l}\text { PJM- } \\
\text { FLON }\end{array}$ & $\begin{array}{l}\text { RL IS } \\
\text { LIFT }\end{array}$ \\
\hline $\begin{array}{l}1976 \\
1975 \\
1974 \\
1973 \\
1972 \\
1971 \\
1970 \\
1969 \\
1968 \\
1967 \\
1966\end{array}$ & $\begin{array}{l}6 \\
5 \\
4 \\
3 \\
2 \\
1 \\
0 \\
9 \\
8 \\
7 \\
6\end{array}$ & $\begin{array}{l}0.0 \\
0.0 \\
0.0 \\
0.0 \\
0.0 \\
0.0 \\
0.0 \\
0.0 \\
0.0 \\
0.0 \\
0.0\end{array}$ & $\begin{array}{r}2.1 \\
2.8 \\
1.3 \\
2.5 \\
3.0 \\
3.0 \\
3.0 \\
3.0 \\
5.0 \\
9.0 \\
10.1\end{array}$ \\
\hline
\end{tabular}

ROD. OF FIELD

$1,019,162$

CODNTY

JON ES

$\begin{array}{ll}\text { D. OF FIELD } & \text { COUNTY } \\ 900.287 & \text { JCNES }\end{array}$

$\begin{array}{rr}\text { OrL-PRD } & E / M D \\ & \\ 519 & 0.7 \\ 1371 & 1.3 \\ 654 & 1.4 \\ 992 & 1.1 \\ 2085 & 1.9 \\ 1916 & 1.7 \\ 2200 & 2 . C \\ 3299 & 3.0 \\ 7012 & 3.7 \\ 11706 & 3.6 \\ 18430 & 5.0\end{array}$

SA S-PRD

16
22
22
24
24
24
24
24
24
34
0

IST DISC AED. FLD NTM PIELD NAHE

DEPTH GRAVITY

CUHOL. PROD. OF FIELD

COUNTY

3,372
540,966

JONES
07? 1955197565823200 NOODLE, NQ. (CANYON SD. 3900)

YEAR

1975
1974
1973
1972
1971
1970
1969
1968
1967
1966

NOR-RELIS

PIOR IIFT

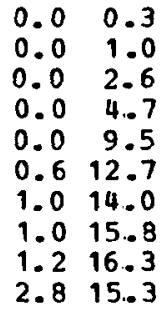

$\begin{array}{ll}0.0 & 0.3 \\ 0.0 & 1.0 \\ 0.0 & 2.6\end{array}$

$0.0 \quad 2.6$

$0.0 \quad 4.07$

0.6 12.7

1.014 .0

1.0

. 2.815 .3
OIL-PED E/ I C GAS-PRD

$\begin{array}{rrr}94 & 0.8 & 3 \\ 1549 & 4.2 & 12 \\ 1632 & 1.7 & 12 \\ 2376 & 1.4 & 19 \\ 3235 & 0.9 & 2690 \\ 7753 & 1.6 & 20382 \\ 11110 & 2.0 & 44219 \\ 12382 & 2.0 & 53129 \\ 14738 & 2.3 & 69791 \\ 21391 & 3.3 & 85410\end{array}$


ABANDONED ONSHORE OIL FIEIDS MITH OVER 250,000 BBL. CUMOLATIVE FRODOCTION

DST DISE ABD. PLD NTM PIELD NAME

07B $1948 \quad 1967 \quad 52047001$ LARGENT

DST DISC ABD. PLD NOM FIELD NAME

079 $1949 \quad 1962 \quad 32453001$ FRAgOSA

DST DISC ABD. FID NTI FIELD NAHE

07D $1940 \quad 1965 \quad 96199001$ STLTH

DST DISC ARD. PID NTM FIELD NAEE

0791949197439668166 HATCHETT (COOK)

$\begin{array}{ccccc}\text { DEPTH } & \text { GRAVITY } & \text { COMUI. } & \text { PROD. OP FIELD } & \text { CCUNTY } \\ 4,676 & 38.0 & 489,502 & \text { JONES }\end{array}$

TEAR

NOM- TELLS

PLOR LIFP

OIL-PRD E/UD

GA S-PRD

1967

0.04 .6

$\begin{array}{lll}10139 & 6.1 & 23204 \\ 14264 & 7.8 & 39239\end{array}$

1966

$0.0 \quad 5.0$

COHOI. PROD. OP PIELD

CCO RTY

DEPTH

GRAVITY

338,256

JON ES

2,936

40.0

DEPTH GRAVITI

COYOL. PROD. OP FIELD

COONTY

2,309

43.1

324,995

JON ES

DEPTH GRAVITY

COMU1. PROD. OF FIELD

COUNTY

2,085

40.0

300,093

JONES

YEAR

1974
1973
1972
1971
1970
1969
1968
1967
1966

NOM-RELIS

PIOR LIFT

OIL-PRD E/RD

GA S-PRD

$\begin{array}{cc}0.0 & 0.1 \\ 0.0 & 0.3\end{array}$

$\begin{array}{ll}0.0 & 0.1 \\ 0.0 & 0.3\end{array}$

0.010 .9

$\begin{array}{ll}0.0 & 2.9\end{array}$

0.02 .0

0.02 .1

$\begin{array}{ll}0.0 & 2.10 \\ 0.0 & 2.7\end{array}$

0.03 .0

$\begin{array}{rr}5 & 0.2 \\ 30 & 0.3 \\ 156 & 0.5 \\ 559 & 0.8 \\ 1180 & 1.6 \\ 1882 & 2.6 \\ 2387 & 3.3 \\ 5810 & 6.0 \\ 7649 & 7.0\end{array}$

0 
ABA NDONED ONSHORE OIL FIELDS RITE OVER 250,000 BBL. COMULATIVE PRODOCTICN

DST DISC ABD. FID NOM FIELD NAHE

07ロ 194919552649001 A NDERSON

DST DISC ABD. FID NOM FIELD NAME.

07म 1955196765855500 NOODIERIRK (ELLENBORGER)

$\begin{array}{lc}\text { DEPTH } & \text { GFAVIT } \\ 2,656 & 38.0\end{array}$

CO MO
L. PROD. OP EIELD
$2 / 8,885$

$$
\begin{aligned}
& \text { NOH-RELIS } \\
& \text { PLON LIFT } \\
& 0.0 \quad 0.3
\end{aligned}
$$

YEAR

1976

DEPTH GRAVITY

5,347

46.0
COHO I. PFOD. OF EIELD

271,048

\begin{tabular}{|c|c|}
\hline & NO: -1 \\
\hline YEAR & FION \\
\hline 1967 & 0.0 \\
\hline
\end{tabular}

COONTY

JON ES

12 FIEID (S)

$\begin{array}{rrr}\text { CIL-PRD } & \text { E/RD } & \text { GAS-PAD } \\ & & \\ 3410 & 5.3 & 2489 \\ 4416 & 6 . C & 4040\end{array}$

OTL-PBD E/

242

2.. 7

COUNTY

JON ES 
ABANDONFD ONSHCRE OII FIEIDS MITH OVER 250,000 BBL. CUMULATIVE PRODDCTION

DST DISC ABD. PLD NIIM FIELD NAME

OTP 1954197178632500 ROWAN 8 HOPE (PENN. REEF)

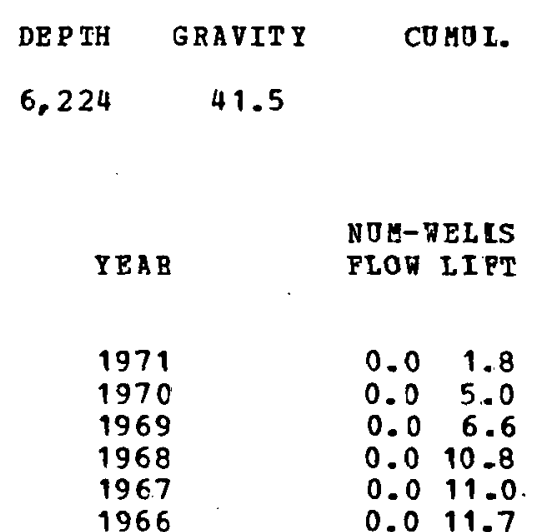

DEPTH

6,741
1966
DST DISC ABN. FLD NOM PIELD NAME

O7R $1955 \quad 1973 \quad 78224500$ ROSCOR (STRANN)
OIL-PRD E/RD GAS-PED

$\begin{array}{rr}640 & 1 . \\ 6546 & 3 . \\ 9733 & 4 . \\ 19870 & 5 . \\ 31813 & 7 \\ 35667 & 8 .\end{array}$

1.0
3.6

3. 6

5. 1

8.4

2440

17448

58865

184575

198561

205909

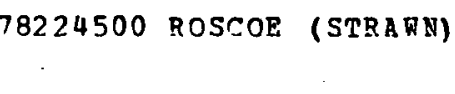

NOH- RELIS

\begin{tabular}{lll} 
YEAR & PION & LIFT \\
& & \\
1973 & 0.0 & 1.8 \\
1972 & 0.0 & 5.0 \\
1971 & 0.0 & 4.9 \\
1970 & 0.0 & 5.0 \\
1969 & 0.1 & 5.0 \\
1968 & 0.6 & 5.0 \\
1967 & 1.0 & 5.0 \\
1966 & 1.5 & 5.0 \\
\hline
\end{tabular}

OIL-PRD E/FD GAS-PRD

$\begin{array}{rrr}3666 & 5.5 & 492 \\ 9685 & 5.3 & 1212 \\ 10958 & 6.1 & 1212 \\ 11232 & 6.2 & 1212 \\ 11747 & 6.3 & 3150 \\ 17740 & 8.7 & 12840 \\ 17702 & 8.1 & 12840 \\ 4561 & 1.9 & 299\end{array}$


ABANDONED ONSRORE OIL FIELDS NITH OVER 250,000 BBL. CUMDLATIVE FRODUCTION

DST DISC ABD. FLD NOM PIELD NAME

O7E $1951 \quad 1976 \quad 26920284$ E. A. (CAMBRIAN SAND)

DEPTH GRAVITY

6,197

YEAR

1976

1975

1974

1973

1972

$19 ? 1$

1970

1969

1968

1967

1966

COUNTY : NOLAN
3 FIEID (S)
COMDI. PROD. OP FIELD COUNTY

286,791

IOL AN

\begin{tabular}{lrrrrr}
\multicolumn{2}{c}{$\begin{array}{c}\text { NOH-GRLIS } \\
\text { FLOW IIFT }\end{array}$} & OIL-PRD & E/RE & GAS-PRD \\
& & & & & \\
976 & 0.0 & 0.3 & 796 & 8.7 & 853 \\
975 & 0.0 & 1.0 & 4151 & 11.4 & 2510 \\
974 & 0.0 & 1.0 & 6305 & 17.3 & 2395 \\
973 & 0.0 & 0.9 & 5882 & 17.6 & 348 \\
972 & 0.0 & 1.0 & 9220 & 25.3 & 48 \\
971 & 0.0 & 1.0 & 8310 & 22.8 & 170 \\
970 & 0.0 & 1.6 & 15987 & 27.7 & 4634 \\
969 & 0.0 & 2.0 & 26949 & 36.9 & 7490 \\
968 & 0.0 & 2.0 & 34683 & 47.5 & 4206 \\
967 & 0.0 & 2.0 & 30375 & 41.6 & 2753 \\
966 & 0.0 & 1.8 & 24293 & 36.3 & 4943
\end{tabular}

$2,253,115$ CUMULATIVE PROD. FROM ABANDONED FIELDS 
ABANDONED ONSHORE OIL PIELDS HITH OVER 250,000 BBL. COMOLATIVE PRODOCTION

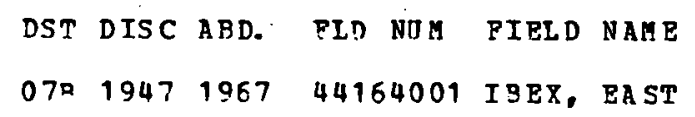

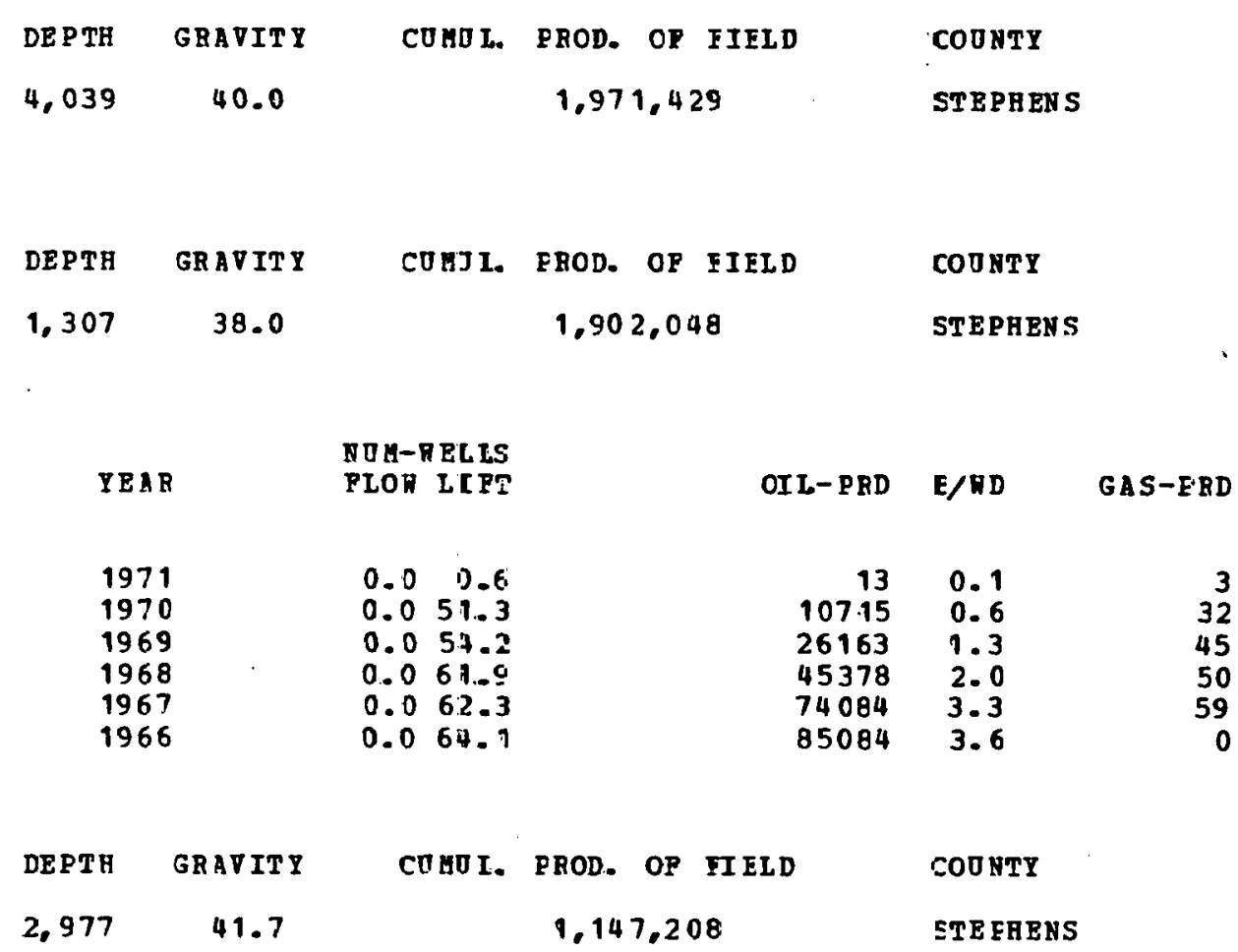

YEAR
1973
1972
1971
1970
1969
1968
1967
1966

$\begin{array}{ll}0.0 & 0.2 \\ 0.0 & 1.0 \\ 0.0 & 1.0 \\ 0.0 & 1.0 \\ 0.0 & 1.0 \\ 0.0 & 1.0 \\ 0.0 & 7.0 \\ 0.0 & 6.6\end{array}$

$\begin{array}{rr}103 & 1.7 \\ 723 & 2.0 \\ 999 & 2.7 \\ 1172 & 3.2 \\ 1384 & 3.8 \\ 1353 & 3.7 \\ 1511 & 0.6 \\ 1914 & 0.8\end{array}$


ABANDONED ONSHORE OIL FIELDS WITH OVER 250,000 BBL. COMULATIVE PRODUCTION

DST DISC AQD. FLD NDM FIELD NAME

0781949196263624500 MUFLLER -O- (ELLENBURGER)

DST DISC ABD. FI.D NDIY FIELD NA ME

$07919541966 \quad 36529332$ RICKELS (CONGLOHERATE 2ND)

DST DISC ABD. FLD VII PTELD NAGE

079 $1947 \quad 1962 \quad 16910001$ CHANEY

DST DISC ABD. PLD NOM FIELD NAME

07B 1950197097921750 TINDHAY (TANNEHILI)

\begin{tabular}{|c|c|c|c|c|c|c|}
\hline DEPTH & GRAVIT Y & COMOL. & PROD. OP & FIEID & CCONTY & \\
\hline 4,145 & 40.0 & & 995,95 & & STE PHENS & \\
\hline DEPTH & TRAVITY & COUOL. & PROD. OP & FIELD & CODNTY & \\
\hline 4.055 & 42.8 & & 662,51 & 2 & STEPBEN S & \\
\hline & R & $\begin{array}{l}\text { NOH-WELIS } \\
\text { PLON LIPT }\end{array}$ & & OIL-PRD & $E / R D$ & GA S-PRD \\
\hline & & $0.0 \quad 0.5$ & - & 420 & 2.3 & 2637 \\
\hline DEPTH & GRAVITY & CUMUL. & PROD. OP & PIEID & CCONTY & \\
\hline 3,365 & 43.0 & & 415.54 & & STE PHENS & \\
\hline DEPTH & GRAVITY & COMOL. & PROD. OF & FIELD & CODNTY & \\
\hline 1,289 & 40.0 & & $41+, 19$ & & STEPHEN S & \\
\hline
\end{tabular}

$\begin{array}{rrrrrr}\text { YERR } & \text { NOM-WEIIS } & \text { OIL-PRD } & \text { E/HD } & \text { GAS-PRD } \\ & \text { FLON LIPT } & & & \\ 1970 & 0.0 & 6.0 & 34 & 0.0 & 8 \\ 1969 & 0.0 & 6.0 & 103 & 0.0 & 12 \\ 1968 & 0.0 & 6.5 & 123 & 0.1 & 12 \\ 1967 & 0.0 & 8.0 & 392 & 0.1 & 45 \\ 1966 & 0.0 & 10.0 & 84 & 0.0 & 0\end{array}$


A AANDONED ONSHORE OII PIELES ATT OVER 250,000 BBL. COAOLATIVE PRODTCTICA

\begin{abstract}
DST DISC ABD. FLD NOM FIELD NAHE O7P $19551970 \quad 10935250$ BOTAR (CADDO)
\end{abstract}

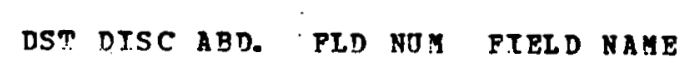

07R. 19431966 . 41446001 BILL

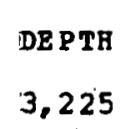

3,225

GRAVITI

42.3

$\operatorname{cog} \mathrm{I}$

PROD. OP EIEID

394,841

COONTY

STEPHEN S

$$
\begin{aligned}
& \text { NOS-RELIS } \\
& \text { FLOR ITPT }
\end{aligned}
$$

OIL-PRD

61
1888

1888
2032

2032
3170

3170
8591

5.2

4.0
3.6

$\begin{array}{cc}\text { CEPTH } & \text { GRAVITY } \\ 4,200 & 44.0 \\ & \\ \text { DZP TH } & \text { GRAVITY } \\ 3.372 & 40.0\end{array}$

COAVL. RROD. OF PIELD

379, 308

COOHTY

STEPBERS

\begin{tabular}{|c|c|c|c|c|}
\hline YEAR & $\begin{array}{l}\text { NOE-DELIS } \\
\text { PLOD IIPT }\end{array}$ & OIL-PRD & E/RD & GAS-PBD \\
\hline 1966 & $0.0 \quad 2.0$ & 465 & D. 6 & $v$ \\
\hline
\end{tabular}

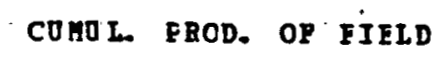

cooxtr

STRPHEH 5 
ABANDONED ONSHORE OIL FIEIDS VITH OVER 250,000 BEL. CIMOLATIUTE PRODOCTION

DST DISC ABD. FLD NDM FIELD NAME

O7B $19421970 \quad 77163001$ ROARK-NAIL

DST DISC BD. FID NJM FIELD NAME

$078 \quad 1947$

98598001 พOODLRY - THOBPE

DST DTSC ABD. PLD NTM PTELD NAME

078 1949196090210800 TIPTON (MISSISSIPPIAN)

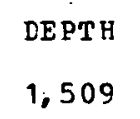

DEPTH

1,509

GRAV IT Y

36.0

COMUI. PROD. OF FIELD

346,361

COUNTY

STE FHENS

$\begin{array}{ll}\text { NOM- RFLLS } \\ \text { PION } \\ \\ \\ 0.0 & \\ 0.0 & 2.1 \\ 0.0 & 4.06 \\ 0.0 & 4.0 \\ 0.0 & .4 .7 \\ 0.0 & 5.0\end{array}$

$\begin{array}{rr}\text { OII-PRD } & E / R C \\ & \\ 1552 & 2.0 \\ 5677 & 3.4 \\ 8742 & 6.0 \\ 22014 & 12.9 \\ 31271 & 17.1\end{array}$

DEPTH GRAVITY

3,925

45.0

CUMUI. PROD. OP FIELD

$\therefore 345,915$

coonty.

STE PHENS

$\begin{array}{cccccc}\text { LEPTH } & \text { GRAVITY } & \text { COMOL. } & \text { PROD . OF PIBLD } & \text { CCUNTY } \\ 4.607 & 45.0 & \therefore & \cdots & 322,208 & \text { STEPHENS }\end{array}$

GAS-PRD

94
318

605

1672 
ABANDONED ONSHOHE OIL FIELDS NITE OVER 250,000 BBL. COUULATIVE PROLUCTION

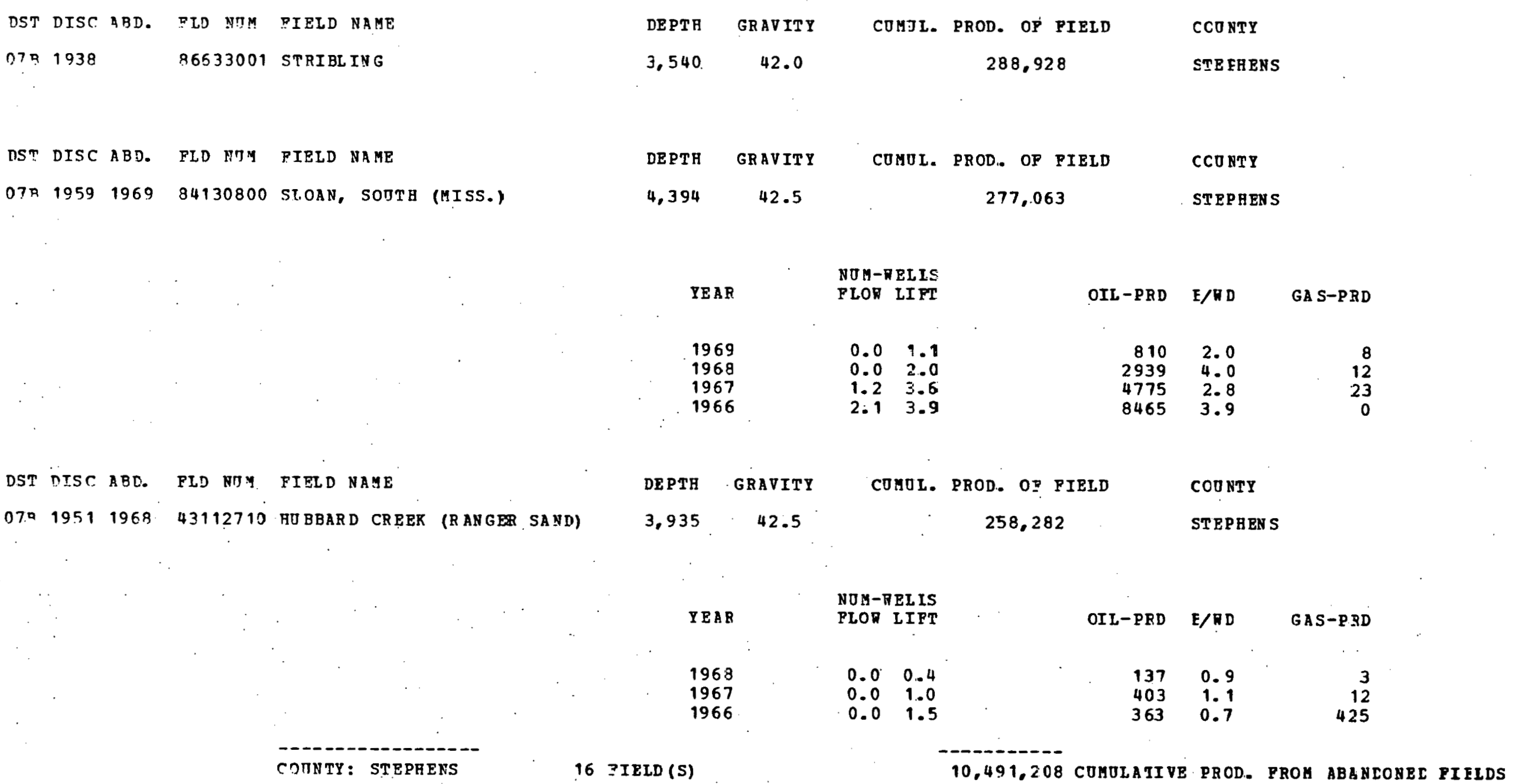


ABARDORED ONSHORE OIL PIELDS $\mathbb{E}$ ITH OVER 250,000 BBL. COMOLATIVE PRODOCTICN

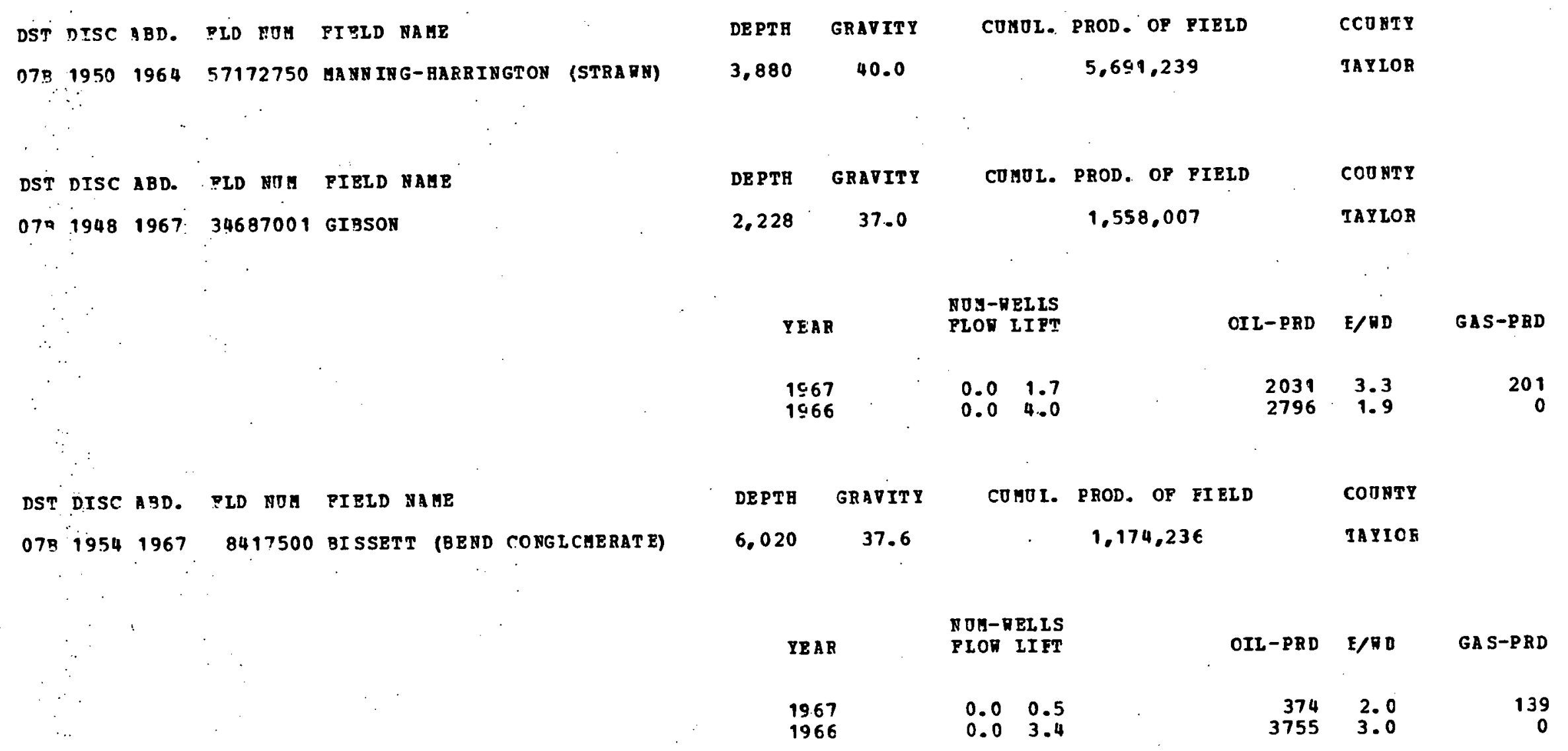


ABANDONED ONSHORE OIL FIRLDS NITH OVER 250,000 BBL. COHOLATIVE PRODOCTION

DST DISC ABD. FLD NMM PTRLD NAME

D $78 \quad 1951$

31250250 FLAT TOP, SOJTH (SHALLON)

DST DISC ABD. PLD NUM PIELD NAME

O7B $19411963 \quad 60666001$ MERKEL

DST DISC ABD. FID NTM PIBLD NAHE

O7D $19501962 \quad 24650009$ DIFKIE

DST DISC ABD. FID NDI FIELD NAHE

$070 \quad 10461957 \quad 63897001$ MPRPHREB

DST DISC AAD. PLD NOM FIEID NABE

O7E 1949196163592001 MUDGE-SLOAR

\begin{tabular}{|c|c|c|c|c|}
\hline DEPTH & GRAVIT Y & CE घOL. & PROD. OP PIELD & CCONTY \\
\hline 2,685 & 40.0 & & 898,802 & TAYICE \\
\hline DEPTH & GRAVITY & COMOL. & PROD. OF FIELD & CCONTY \\
\hline 2,591 & 38.0 & & 736,585 & TAYLOR \\
\hline DB P TH & GRA VITI & conoI. & PROD. OF FIELD & COONTI \\
\hline 4,470 & 41.0 & & 574,930 & TAYIOR \\
\hline DEPTH & GRAVITY & cosot. & PAOD. OP FIELD & COUNTY \\
\hline 3.062 & 41.0 & & 561,024 & TAYLOR \\
\hline DEPTH & GRAVIT Y & CO $90 \mathrm{~L}$. & PROD. OF FIEID & COONTY \\
\hline 4,498 & 40.0 & & 554.707 & TAYIOE \\
\hline
\end{tabular}


ARANDONED ONSHORE OIL PIELDS WITH OVER 250,000 BEL. CUMULATIVE FRODOCTICN

DST DISC ABD. FLD NOM PIELD NAME

$\begin{array}{lllll}07 R & 1956 \quad 1973 \quad 81436750 & \text { SCHICR (STRAWN) }\end{array}$

DST DISC ABD. PLD NDM PIRLD NAME

$079 \quad 1953 \quad 1970 \quad 13225250$ B.OLI (GARDUER)

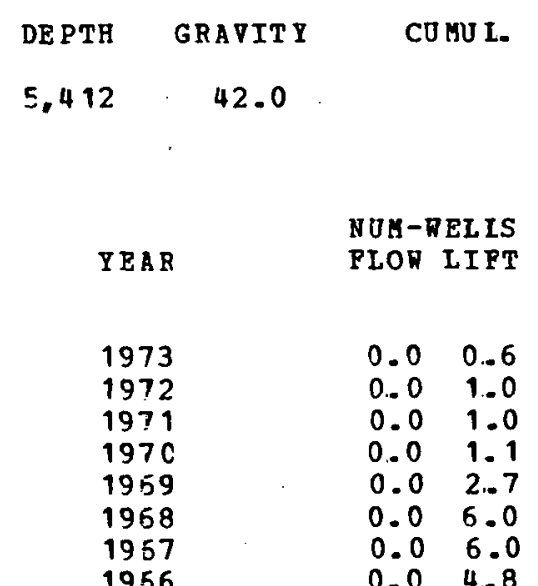

P ROD

$\begin{array}{ll} & \text { COONTY } \\ 535.028 & \text { TAYLOR }\end{array}$

\author{
DEPTH GRAVITY
}

4,274

39.0

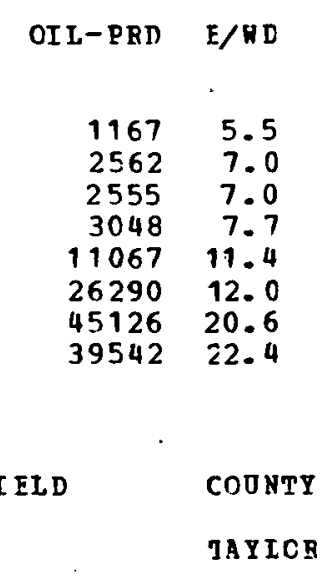

492,162

OIL-PRD E/WD

GA S-PRD

$\begin{array}{rrr}1334 & 2.0 & 636 \\ 1.840 & 2.5 & 1260 \\ 1801 & 2.5 & 1260 \\ 1880 & 1.9 & 1776 \\ 3966 & 2.5 & 0\end{array}$

GAS-PRD

52

558

1228

1662

5121

7915

6278
NOR-HELLS

FION LIFT

$$
\begin{array}{ll}
0.0 & 1.8 \\
0.0 & 2.0 \\
0.0 & 2.0 \\
0.0 & 2.7 \\
0.0 & 4.3
\end{array}
$$


ABY NDONED ONSHORE OII FIEEDS NITH OVBR 250,000 BBL. COHOLATIVE PRODUCTICY

$\begin{array}{lllll}\text { DST DISC ABD. } & \text { PID NNM } & \text { PIBLD NAHE } \\ \text { O7म } & 1953 & 1974 & 20803750 & \text { CORSICA } \\ \text { (STRARN) }\end{array}$

DST DISC ABD. PLD NJM PIBLD NAME

D7D 1954 1970.90984750 TRAVIS (STRARN 3300)

\begin{abstract}
078 1953 1974 20803750 CORSICA (STRAMN)
\end{abstract}

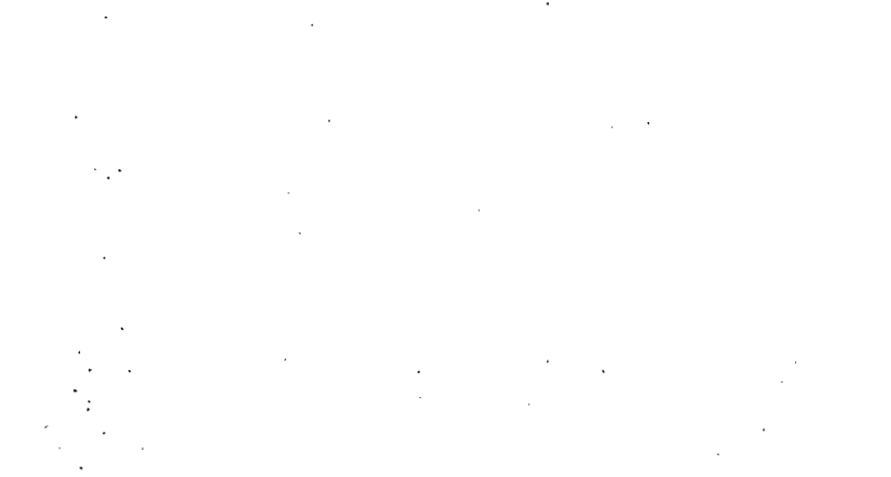

$\begin{array}{ccccc}\text { DEPTH } & \text { GRAVITY } & \text { COLOI. } & \text { PROD. OF FIELD } & \text { COONTY } \\ 5,182 & 40.9 & & 483,793 & \text { TAYLOR }\end{array}$

$\begin{array}{lrrrrr}\text { YEAR } & \begin{array}{r}\text { MDU-RELIS } \\ \text { PLOR }\end{array} & \text { OIFT } & & \\ & & & & & \\ 1974 & 0.1 & 0.0 & 45 & 1.5 & 4 \\ 1973 & 0.1 & 0.9 & 3720 & 10.2 & 503 \\ 1972 & 0.0 & 1.3 & 3382 & 6.9 & 3202 \\ 1971 & 0.0 & 2.2 & 6759 & 8.5 & 6224 \\ 1970 & 0.0 & 3.0 & 8301 & 7.6 & 5761 \\ 1969 & 0.0 & 3.0 & 10065 & 9.2 & 10848 \\ 1968 & 0.0 & 3.0 & 9569 & 8.7 & 13426 \\ 1967 & 0.0 & 2.3 & 13476 & 16.4 & 13764 \\ 1966 & 0.0 & 3.0 & 13256 & 12.1 & 20273\end{array}$

$\begin{array}{ccccc}\text { DEPTH } & \text { GRAVITY } & \text { COUUI. } & \text { PROD. OF PIELD } & \text { CODRTY } \\ 3,349 & 38.3 & & 479,007 & \text { TAYLOR }\end{array}$

IEAR
1970
1969
1968
1967
1966


ABANDONED ONSHORE OII FIELDS MITH OVER 250,000 BBL. CUGULATIVE PRODUCTION

DST DISC ABD. PID NOB FIELD NAME

07B 1954196896861800 ดหITE (TAN NEHILL)

DST DISC ABD. FLD NOM. FIELD HAHE

0781957 1971. 4359500 ATDRA (FRY)

DEPTH GRAVIT

2,735

39.2

YEAR

1968

1967

1966
COMOI. PROD. OP FIELD

448,126

COUNTY

IA YLC F

NOM-VELIS
PLON LIFT

$\begin{array}{ll}0.0 & 1.8 \\ 0.0 & 3.0 \\ 0.0 & 3.0\end{array}$

OIL-PRD E/RC

GA S-P RD

$\begin{array}{rr}862 & 1.3 \\ 3891 & 3.6 \\ 2425 & 2.2\end{array}$

COONTY

TAYIOR
COHU1. PROD. OF FIELD

402.543
707

23
4. 198

41.0

NOH-RELIS

YEAR

1971

1970

1969

1968

1967

1966
PLOW LIFT

0.0 .2 .0

$0.0 \quad 5.1$

0.0 .6 .2

0.07 .0

$0.0: 7.0$
OIL-PRD E/RD GAS-PRD

$\begin{array}{lll}366 & 0.5 & 1665\end{array}$

$1932 \quad 1.0 \quad 5458$

$4259 \quad 1.9 \quad 3907$

$\begin{array}{lll}5768 & 2.3 & .3640\end{array}$

$\begin{array}{lll}5813 & 2.3 & 3497\end{array}$

$\begin{array}{lll}7385 & 2.9 & 7256\end{array}$ 
A AANDONED ONSHORE OIZ FIZLDS MITH OVER 250,000 BBL. CTHOLATIVE PRODOCTION

DST DISC ARD. FLO NUM FTELD HAME

O7 B-1950 197349580001 KIOWA PEAR

DST DISC ABD. FLD NIM PIRLD NAME

O7P. $1944 \quad 1973 \quad 29753001$ EWALT

$\begin{array}{cc}\text { DEPTH } & \text { GRAVITY } \\ 5,285 & 37.0\end{array}$

COMOL. PROD. OP FIELD

377.598

COUNTY

TAYIOB

\begin{tabular}{lll} 
& \multicolumn{2}{l}{ NOM- YELES } \\
& FLON LIET \\
& & \\
1973 & 0.0 & 0.8 \\
1972 & 0.0 & 1.0 \\
1971 & 0.0 & 1.0 \\
1970 & 0.0 & 1.0 \\
1969 & 0.0 & 1.0 \\
1968 & 0.0 & 1.0 \\
1967 & 0.0 & 1.1 \\
1966 & 0.0 & 2.0
\end{tabular}

DEPTH GRAVITY

4,152

40.0

COMUL. PROD. OF PIELD

344,581

$\begin{array}{rr}\text { OIL-PRD } & E / \text { R D } \\ & \\ 2938 & 9.7 \\ 3916 & 10.7 \\ 3871 & 10.6 \\ 3177 & 8.7 \\ 3637 & 10.0 \\ 3700 & 10.1 \\ 5327 & 13.5 \\ 15930 & 21.8\end{array}$

GA S-PRD

384

513

. 511

864
1654

290

13
0

Y E A R

NUM-RELIS

PLON LIFT

$0.0 \quad 0.7$

0.01 .0

$0.0 \quad 9.0$

0.001 .7

0.02 .0

0.02 .0

0.81 .8

1.02 .0

$\begin{array}{rrr}\text { CIL-PRD } & \text { E/WD } & \text { GAS-PBD } \\ & & \\ 615 & 2.5 & 9 \\ 1234 & 3.4 & 12 \\ 2133 & 5.8 & 12 \\ 353 & 0.6 & 12 \\ 2748 & 3.8 & 21 \\ 3327 & 4.6 & 24 \\ 2937 & 3.1 & 6107 \\ 4584 & 4.2 & 12192\end{array}$


A BA NDONRD OASHORE OIL PIEIDS ITH OPER 250,000 BBL. COMULATIVE PRODECTICY

DST DISC ABD. PLD NTM FIELD NAMB

$0701954 \cdot 197096961200$ REITE (FAMP COLORADO)

DST DISC ABD. PLD ROA PIELD NAKE.

07P $1956 \quad 1969 \quad 40546656$ RERBRR (GRAY SAYD)

\begin{tabular}{|c|c|c|}
\hline DEPTH & GRAVITI & CO L L L \\
\hline 2,494 & 44.0 & . \\
\hline $78 A R$ & & $\begin{array}{l}\text { NOA-NELIS } \\
\text { PIOH IIFT }\end{array}$ \\
\hline $\begin{array}{l}1970 \\
1969 \\
1968 \\
1967 \\
1966\end{array}$ & & $\begin{array}{ll}0.0 & 0.3 \\
0.0 & 1.0 \\
0.0 & 2.7 \\
0.0 & 5.0 \\
0.0 & 5.0\end{array}$ \\
\hline
\end{tabular}

$\begin{array}{cc}\text { DEPTE } & \text { GRAVITI } \\ 4.901 & 44.0\end{array}$

COHOL. PROD: OP PIELD

326,741

$\begin{array}{rrr}\text { OIL-P RD } & \text { E/MD } \\ & & \\ & 154 & 1.3 \\ 1660 & 4.5 \\ 2681 & 2.8 \\ 5998 & 3.3 \\ 4818 & 2.6\end{array}$

GAS-PED

600

3150

1212

36
0
COORTY

IAYIOR
TEAR

1969

1969
1968

1967

1966
NOA-RELIS
PLOR. LIFT

$$
\begin{array}{ll}
0.0 & 1.0 \\
0.0 & 1.0 \\
0.0 & 1.0 \\
0.0 & 1.0
\end{array}
$$

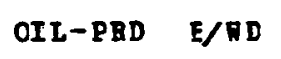

$\begin{array}{rr}667 & 1.8 \\ 1969 & 5.4\end{array}$

$2587 \quad 7.9$

$2999 \quad 8.2$
GAS-PBD

91

1039

7386 
ABANDONED ONSHORE OIL FIELDS NITH OVER 250,000 EBL. COMOLATIVE PRODUCTICN

DST DISC ABD. FID NTM FIELD NAGE

$07819541971 \quad 7409500$ BERTA (BEND CONGLOMERATE)

DST DISC ABD. FLD NOM FIELD NAME

O7B 1962197432654166 FRANKIRK, EAST (CADDO)

DEPTH
$E, 288$
YEAR
1971
1970
1969
1968
1967
1966

\section{DEPTH \\ GRAVITI}

5.395

39.0

YEAR

WJM-WELLS
FLON LIFT

1974

1973

1972

1971

1970

1969

1968

1967

1966

FLY W ELIS

$0.0 \quad 1.7$

$0.0 \quad 2.0$

$0.0 \quad 2.0$

0.0 2..0

$\begin{array}{ll}0.0 & 2.0 \\ 0.3 & 1.8\end{array}$
GR A VIT Y

38.0

CUMU I. FROD. OF FIELD

321,306

COUNTY

TAY LOR
CUMO 1. FROD. OF FIELD

303,884

CIL-PRD E/RD GAS-PED

$12712.1 \quad 120$

$\begin{array}{lll}3432 & 4.7 & 1319\end{array}$

6.5

54277.4

$\begin{array}{rrr}5427 & 1.4 & 6284 \\ 6768 & 9.3 & 0\end{array}$

$\begin{array}{lll}0.0 & 3.0 \\ 1966 & 0.0 & 2.7\end{array}$

$\begin{array}{ll}0.0 & 0.7 \\ 0.0 & 2.0 \\ 0.0 & 2.1 \\ 0.0 & 2.3 \\ 0.0 & 3.0 \\ 0.0 & 3.0 \\ 0.0 & 3.0 \\ 0.0 & 3.0 \\ 0.0 & 2.7\end{array}$

$E /$ R

GA S-PRD

$\begin{array}{rrr}3308 & 13.6 & 4910 \\ 9123 & 12.5 & 14295 \\ 12230 & 16.8 & 22971 \\ 13562 & 13.5 & 3144 \epsilon \\ 17502 & 16.0 & 32865 \\ 21123 & 19.3 & 39940 \\ 25006 & 22.8 & \equiv 5199 \\ 24475 & 22.4 & 25313 \\ 24053 & 24.0 & \Xi 1969\end{array}$


ABANDONED ONSHORE OIL FIELDS WITH OVZR 250,000 BBL. COMULATIVE FRODOCTICN

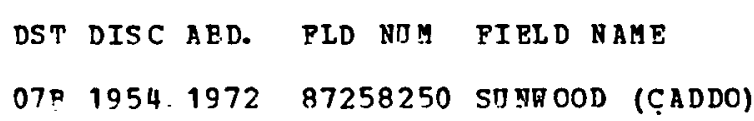

\begin{tabular}{|c|c|c|c|}
\hline DEPTH & GBAVIT Y & \multicolumn{2}{|c|}{ CO MO L. } \\
\hline 4.203 & 41.2 & & \\
\hline IEAR & & $\begin{array}{l}\text { NOM-1 } \\
\text { PLON }\end{array}$ & $\begin{array}{l}\text { EL IS } \\
\text { LIFT }\end{array}$ \\
\hline $\begin{array}{l}1972 \\
1971 \\
1970 \\
1969 \\
1968 \\
1967 \\
1966\end{array}$ & & $\begin{array}{l}0.0 \\
0.0 \\
0.0 \\
0.0 \\
0.0 \\
0.0 \\
0.0\end{array}$ & $\begin{array}{l}1.0 \\
1.0 \\
1.0 \\
1.5 \\
2.0 \\
2.0 \\
2.8\end{array}$ \\
\hline
\end{tabular}

\section{DEPTH GRAVITY}

4. 367

42.0

\section{NOR-NELIS}

PLOD LIFT

1975

1974

1973

1972

1971

1969

1968

1967

1966

$$
\begin{array}{ll}
0.1 & 0.2 \\
1.0 & 0.0 \\
1.0 & 0.0 \\
1.0 & 0.0 \\
1.0 & 0.0 \\
1.0 & 0.0 \\
1.0 & 0.0 \\
1.0 & 0.0 \\
1.0 & 0.0 \\
1.0 & 0.0
\end{array}
$$

CONTY

TATIOR

$$
287,311
$$

$\begin{array}{rr}\text { CII-PRD } & \text { E/RI } \\ 1268 & 3.5 \\ 1264 & 3.5 \\ 2340 & 6.4 \\ 1976 & 3.6 \\ 1897 & 2.6 \\ 3638 & 5.0 \\ 4705 & 4.5\end{array}$

GAS-PRD

COONTY

TAYIOR

31
11
23
18
31
35
0


ABANDONRD ONSHORE OIL FIEIDS MITA OVER 250,000 BBL. COUDLATIVE PROLOCTION

NST DISC ABD. FLD NOY FIELD NAME

$078 \quad 19421967 \quad 91040001$ TRENT

DST. DISC ABD. FID NDE FIELD NABE

0791961197591498426 TROY (CADDO, OPPER -8-)

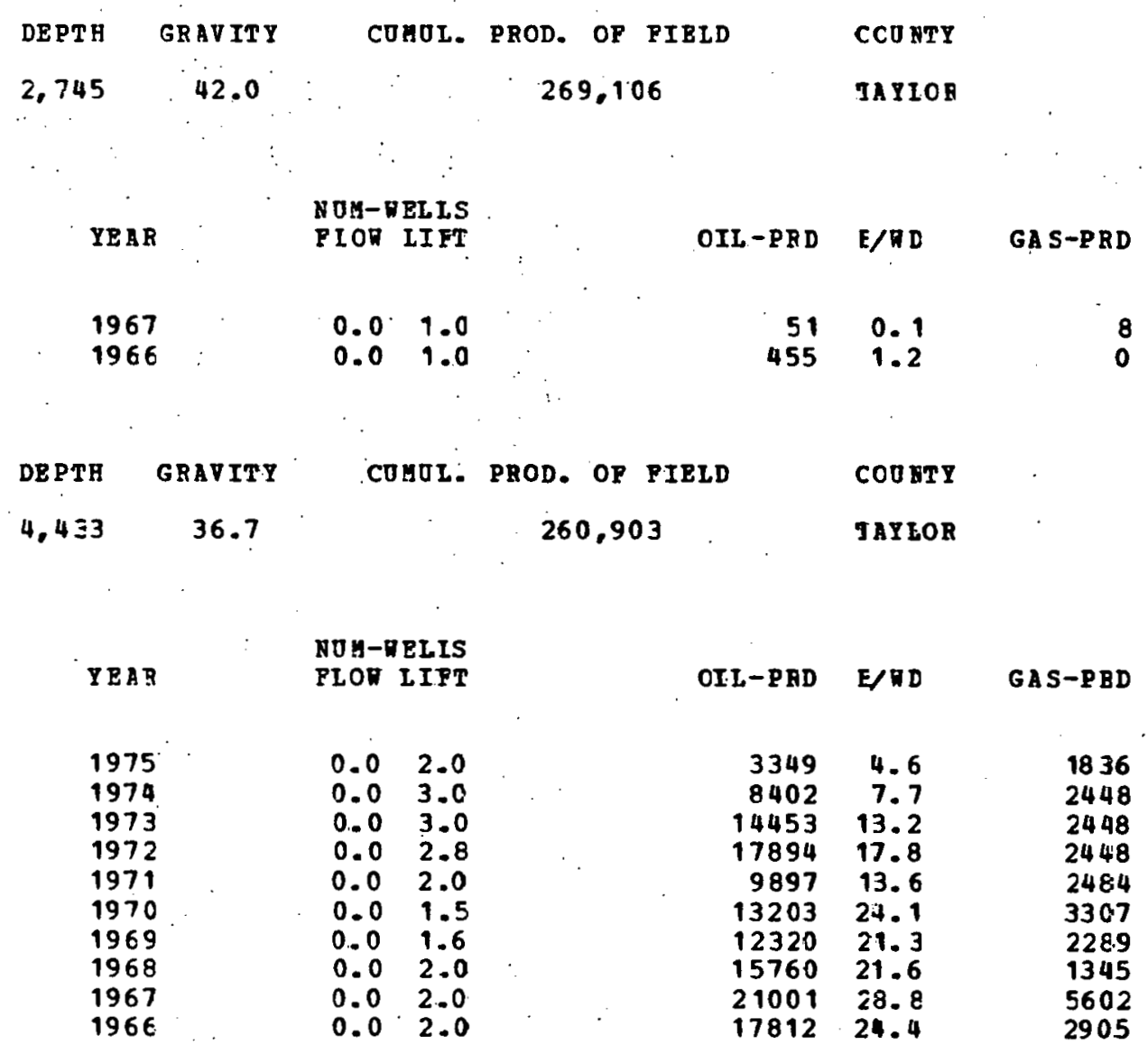

24 FIELD (S)

$17,690,625$ COHULATIVE PRCD. PROM ABANCONBD PIELDS

48,202,949 COMULATIVE PROD. PROM ABANDONED PIEIDS 
APA NDONED OHSHORE OIL PIELDS ITH OVER 250,000 BBL COMULATIVE PRODDCTICN

DST DISC.ABD.: FLD TYM FIPLD VIAGE

O7C $1952 \quad 1973 \quad 12175852$. BRONTE (4800 SAN D)

DST DISC ABD. FID NOM FIELD NAME

07ᄃ 1953196612175426 BRONTE (ELLEN BUR GER)

\begin{tabular}{|c|c|c|c|}
\hline OE PT & GRAV IT $Y$ & \multicolumn{2}{|c|}{ COHOL: } \\
\hline 4,838 & 45.0 & & \\
\hline & & NOM-n & EL LS \\
\hline YEAR & & FLOH & LI PT \\
\hline $\begin{array}{l}1973 \\
1972 \\
1971 \\
1970 \\
1969 \\
1968 \\
1967 \\
1956\end{array}$ & & $\begin{array}{r}0.0 \\
0.0 \\
0.0 \\
13.4 \\
18.7 \\
21.8 \\
22.0 \\
15.3\end{array}$ & $\begin{array}{l}0.6 \\
3.7 \\
4.6 \\
4.8 \\
5.1 \\
3.2 \\
3.0 \\
1.1\end{array}$ \\
\hline
\end{tabular}

DE PTH

GRAVITY

5,700

49.2

COMUL. PROD. OE FIELD

286,422

OIL-PRD E/RE GAS-PRD

$\begin{array}{rr}258 & 1 . \\ 9525 & 7 . \\ 33819 & 20 . \\ 104756 & 15 . \\ 401445 & 46 . \\ 457971 & 50 . \\ 416141 & 45 . \\ 324977 & 54 .\end{array}$

1.2
7.1
20.2
15.8
46.3
50.2
45.6
54.2

990

12678

48606

225125

898925

441951

303853

292051

YEA

NOM-WEILIS

FLOW LIFT

OIL-PRD E/WD

GAS-P BD

$0.0 \quad 0.9$

$4115 \quad 12.3$

0 
ABANDONED ONSHORE OII FIIIDS UITH OVER 250,000 BBL. CJMOLATIVE FRODUCTION

DST DTSC ABD, PLD NDM PIELD NAME 07C· 1953196674877600 RAWLINGS (4500)

$\begin{array}{ccccc}\text { DEPTH } & \text { GRAVITY } & \text { COMOL. } & \text { PROD. OF PIELD } & \text { CCONTY } \\ 4.500 & 44.5 & & 267.517 & \text { CORE }\end{array}$

YE A R

NOM-WELIS PLOW LIFT

$\begin{array}{rrr}\text { OII-PRD } & \text { E/HD } & \text { GAS-PED } \\ 2943 & 3.2 & 857\end{array}$

$0.0 \quad 2.5$

$6,628,966$ COMULATIVE 2ROD. FROM ABANDONED FIEIDS
COJNTY : COKE

3 FIELD (S) 
ABANDONED ONSHORE OIL FIEIDS HITH OVER 250,000 BBL. COUULATIVE FROLUCTION

DST DISC ABD. PLD NTM FIELD NAME

O7C 1964197429292200 ESCONDIDO (ELIENPORGER)

DST DISC ABD. FLD NOM PIELD NAHE

O7C 1963196629292600 ESCONDIDO (PENN, LONER)

$\begin{array}{ccccc}\text { DE PTH } & \text { GRAVITY } & \text { COMOI. } & \text { PROD. OF FIELD } & \text { COONTY } \\ 9,043 & 40.6 & 979,179 & \text { CROCRETT }\end{array}$

YEAR

NOM-TELIS

PLOD LIFT

OIL-PRD E/WD

GAS-PRD

1974

1973

1972

1971

1970

1969

1968

1967

$\begin{array}{ll}0.0 & 0.1 \\ 0.0 & 1.0 \\ 0.0 & 1.0 \\ 0.0 & 2.2 \\ 0.6 & 3.3 \\ 1.0 & 3.9 \\ 1.0 & 4.0 \\ 1.7 & 4.0 \\ 2.5 & 3.0\end{array}$

131443.2

1857350.9

2934280.4

4477954.5

$38066 \quad 26.6$

$47964 \quad 26.7$

$\begin{array}{ll}47964 & 26.7 \\ 73920 & 40.5\end{array}$

$\begin{array}{rr}73920 & 40.5 \\ 180437 & 87.2\end{array}$

$255301 \quad 127.2$

\section{0}

35297

17311

119581

119581

61390

35720

40845

38132

$\begin{array}{ccccc}\text { DEPTH } & \text { GRAVITY } & \text { CONOL. } & \text { PROD. OF FIELD } & \text { COONTY } \\ 8,306 & 45.8 & & 346,329 & \text { CROCRETT }\end{array}$

\begin{tabular}{|c|c|c|c|c|}
\hline YEAR & $\begin{array}{l}\text { NUM-NELIS } \\
\text { FLOR LIFT }\end{array}$ & OIL-PRD & E/HE & GAS-PRD \\
\hline 1966 & $0.8 \quad 0.0$ & 22607 & 74.3 & 24051 \\
\hline
\end{tabular}


ABANDONED ONSHORE OEL FIELDS WITH OVER 250,000 BBL. CUMULATIVE PRODUCTION

DST DJSC ABD. PLD NTM FIELD NABE

O7C 1967197429296333 ESCONDIDO, NH. (ELLEN.)

$\begin{array}{ccccc}\text { DEPTH } & \text { GRAVITY } & \text { COEOL. } & \text { PROD. OP FIELD } & \text { CCORTY } \\ 9,400 & 45.0 & 263,997 & \text { CROCKETT }\end{array}$

YEAR

NDM-RELIS

PION IIFT

OIL-PRD E/RD

GA S-P RD

$0.0 \quad 0.5$

0.01 .0

0.01 .0

0.01 .0

0.01 .0

0.01 .0

0.01 .0

$0.0 \quad 0.5$

1968

1967

COONTY: CROCKETT

3 PIELD (S)

$\begin{array}{rrr}4847 & 26.6 & 5289 \\ 13425 & 36.8 & 6658 \\ 32521 & 89.1 & 3210 \\ 28040 & 76.8 & 2686 \\ 35399 & 97.0 & 3499 \\ 53180 & 145.7 & 5309 \\ 61795 & 169.3 & 6134 \\ 34790 & 190.6 & 3479\end{array}$

$1,-0-0 .--0$

1,589,505 COHDLATIVE PROD. PROA ABAHCORED PIELDS 
ABANDONED ONSHORE OIL PIELDS ITH OVER 250,000 BBL. COHULATIVE PRODDCTICN

DST DISC ABD. FID NTIS PTELD NAME

07C 1955196986115400 STILES (ELLENBURGER)

NST DISC ABD. PI.D NTI PIELD NAME

O7C 195319757927200 BIG LARE, W. (ELIENBORGER)

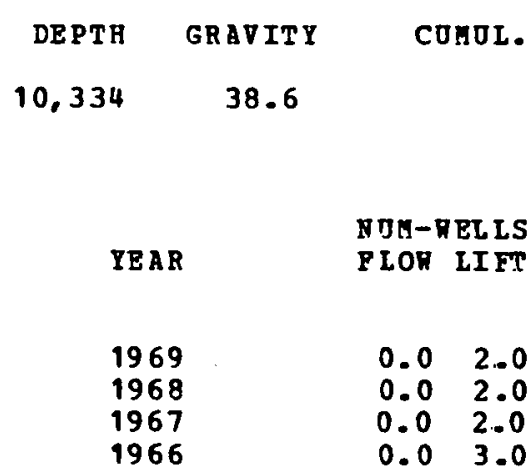

DE PTH GRAVITY

9,240

YEAR

1975

1974

1973

1972

1971

1970

1969

1968

1967

1966

COUNTY : REAGAN $\operatorname{cov} \operatorname{sT}$

EEAGA

$$
727,056
$$

$\begin{array}{rrr}\text { OIL-PRD } & \text { E/RD } & \text { GAS-PRD } \\ & & \\ 5020 & 6.9 & 28203 \\ 4527 & 6.2 & 32590 \\ 6054 & 8.3 & 28957 \\ 9000 & 8.2 & 40982\end{array}$

COHOL. PROD. OP PIELD

COONTY

422,061

FBAGAN

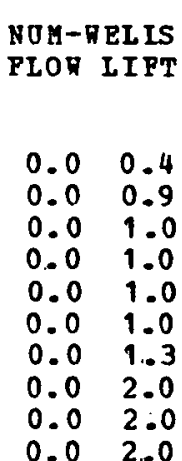

GAS-PBD

$\begin{array}{rr}65 & 0.4 \\ 1758 & 5.3 \\ 5671 & 15.5 \\ 5660 & 15.5 \\ 7038 & 19.3 \\ 6512 & 17.8 \\ 7752 & 15.9 \\ 8571 & 11.7 \\ 9176 & 12.6 \\ 8548 & 11.7\end{array}$

$1,149,117$ COMOLATIVE PROD. PROM ABANDONED FIELDS 
ABANDONED ONSHORE OIL FIELDS UITA OVER 250,000 BBL. COMOLATIVE ERODOCTION

DST DISC ABD. FLD NOM FIELD NAME

O7r 1952197221735625 CRERS, SCOTH (GAFDNER, OP.)

$\begin{array}{cc}\text { DEPTH } & \text { GRAVITI } \\ 3,835 & 44.0\end{array}$

3,835

YEAR

1972

1971

1970

1969

1968

1967

1966

DST DISC ABD. FLD NOM FIELD NAME

O7C $9954 \quad 1970 \quad 80728333$ SANPORD (PRY)

\section{DEPTH GRAVITY}

4,518

40.6
COMU1. PROD. OP FIELD

$$
2,145,022
$$

CODNTI

RON NELS

\begin{tabular}{|c|c|c|c|c|}
\hline YEAR & $\begin{array}{l}\text { NOM-KELIS } \\
\text { PLOW LIFT }\end{array}$ & CIL-P BD & $E / H D$ & GAS-PAD \\
\hline $\begin{array}{l}1970 \\
1969 \\
1968 \\
1967 \\
1966\end{array}$ & $\begin{array}{rr}0.0 & 0.5 \\
0.0 & 3.3 \\
0.0 & 10.0 \\
0.0 & 45.4 \\
0.0 & 72.8\end{array}$ & $\begin{array}{r}82 \\
7478 \\
15675 \\
65187 \\
185456\end{array}$ & $\begin{array}{l}0.4 \\
6.3 \\
4.3 \\
3.9 \\
7.0\end{array}$ & $\begin{array}{r}2 \\
232 \\
488 \\
2700 \\
18010\end{array}$ \\
\hline
\end{tabular}


ABANDONED ONSHORE OIL FIELDS WITH OTER 250,000 BBL. COMOLATIVE FRODUCTION

DST DISC ABD. FLD NOM FIELD NAME

07C $19521971 \quad 86932500$ SUEI SE (PRY SAND)

DST DISC ABD. FID NOM FIELD NAME

07C 1950197065883250 NORA (GARDNER SAND)

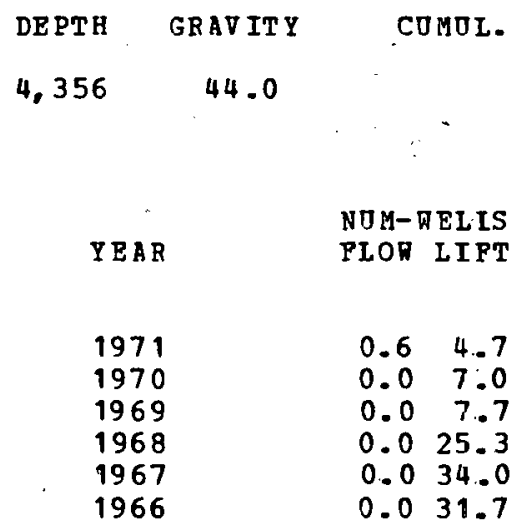

PROD. OP FIELD

CCONTY

$1,969,368$

RON N ELS

$\begin{array}{lcc}\text { DEPTH } & \text { GRAVITY } & \text { CUMOI. } \\ 4.062 & 46.8 & \\ & & \\ & & \text { NOM- AELIS } \\ \text { YEAR } & \text { FIOR LIFT } \\ & \\ 1970 & 0.0 \quad 0.9 \\ 1967 & 0.00 .0 \\ 1966 & & 0.010 .9\end{array}$

OIL-PRD
968
7172
20354
57213
90741
15485

WD
0.5
2.8
7.3
6.2
7.3
1.3

COONT.Y

FON NEIS
G A S - P BD

7
46
177
36
34
1312

$$
1,726,275
$$

OIL-PRD E/RD

GA S-PRD

$1164 \quad 3.5 \quad 8$

$\begin{array}{lll}4432 & 0.0 & 11783\end{array}$ 
ABANDONFD ONSHCRE OI= FIEIDS RITH CVER 250,000 BBL: COMOLATIVE PRODUCTICN

DST DISC ABD. FID NTM FIELD NAME

07C 1952197421735375 CREWS, SOUTH (FRY, LO.)

DST DISC ABD. FLD NOM PIELD NAME

O7r $1951 \quad 1975 \quad 53657664$ IINDEMANN (ICMILIAN SD)

\begin{tabular}{|c|c|c|c|}
\hline DEPTH & GRAVITY & \multicolumn{2}{|c|}{ CO HE L. } \\
\hline 3,621 & 44.2 & & \\
\hline YEAR & & $\begin{array}{l}\text { NOM- } \\
\text { PLOW }\end{array}$ & $\begin{array}{l}\text { ELIS } \\
\text { LIFT }\end{array}$ \\
\hline $\begin{array}{l}1974 \\
1973 \\
1972 \\
1971 \\
1970 \\
1969 \\
1968 \\
1967 \\
1966\end{array}$ & & $\begin{array}{l}0.0 \\
0.0 \\
0.0 \\
0.0 \\
0.0 \\
0.0 \\
0.0 \\
0.0 \\
0.0\end{array}$ & $\begin{array}{l}0.5 \\
1.0 \\
1.0 \\
1.0 \\
1.0 \\
1.0 \\
1.0 \\
1.0 \\
7.2\end{array}$ \\
\hline
\end{tabular}

ROD. OF FIEL

$1,318,248$

COONTY

GON NELS

\begin{tabular}{|c|c|c|c|}
\hline DE PTH & GRAVITY & CU MO:L. & PROD. OP FIEID \\
\hline 2,46 & 43.4 & & $1,196,948$ \\
\hline
\end{tabular}

$1,196,948$
NOH-VELIS

PLON LIPT

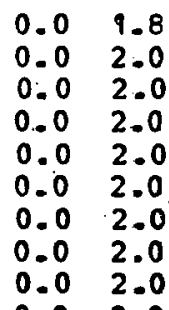

$0.0 \quad 2.0$

1975

1973

1972

1971

1970

1969

1966
GAS-PRD

$\begin{array}{rrr}1116 & 6.1 & 8 \\ 2172 & 6.0 & 12 \\ 2306 & 6.3 & 12 \\ 2534 & 6.9 & 12 \\ 2325 & 6.4 & 12 \\ 2248 & 6.2 & 12 \\ 2351 & 6.4 & 450 \\ 2647 & 7.3 & 794 \\ 3020 & 1.2 & 0\end{array}$

RON NELS 
ABANDONED ONSHORE OIL FIELDS ITH ORER 250,000 BBL. COYULATIVE PRODUCTICN

DST DISC ABD. FLD NDM FIELD NAME

O7C $1927 \quad 1953 \quad 59801001$ MCMIILAN

DST DISC ABD. FLD NIT FIELD NAME

O7C 1952196368271500 PACE (PRY SAND)

DST DISC.ABD. FLD NOM TIELD NAME

O7r $19501970 \quad 87792666 \quad$ SYKES (HORRIS)

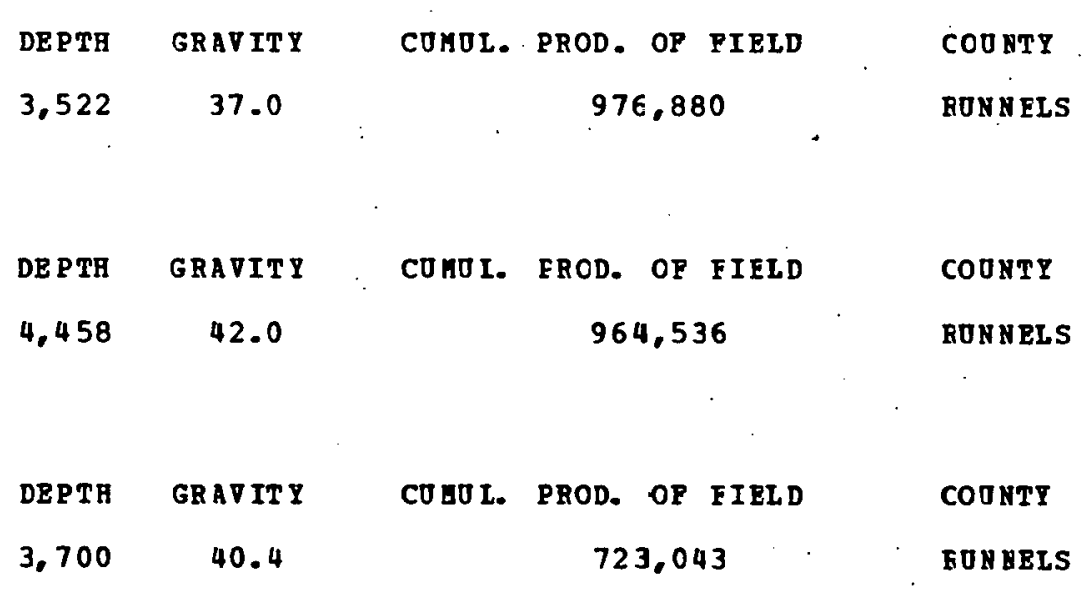

YBAR

1970
1969
1968
1967
1966

MUR-RELLS

OIL-PBD E/RE GAS-PRD

0.01 .3

0.08 .0

$0.0 \quad 8.0$

0.0 .8 .0

0.010 .0

$\begin{array}{rrr}243 & 0.5 & 2 \\ 3857 & 1.3 & 2870 \\ 5839 & 2.0 & 7499 \\ 12342 & 4.2 & 11318 \\ 17761 & 4.9 & 0\end{array}$


ABANDONED ONSHORE OIL FIELDS RITH OVER 250,000 BBL.. CUUOLATIVE PRODUCTICN

DST DISC ABD. PLD NDM PIELD NAME

O7C 1951197169640001 PATSY

DST DISC ABD. FLD NIE FIELD YAME

O7C $1951 \quad 1970 \quad 98182071$ NINTERS (CADDC)

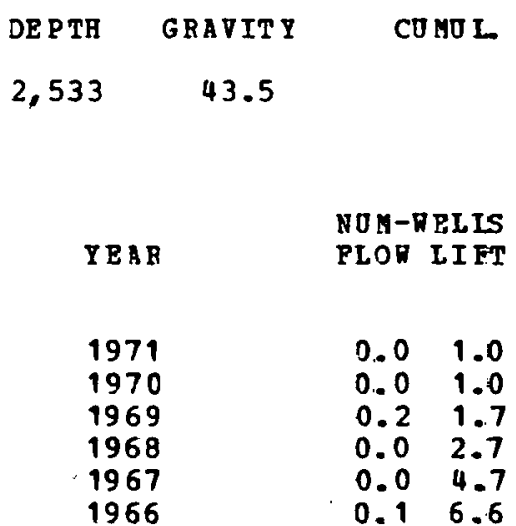

DEPTH GRAVIT Y

4,516

46.0

CDHOI. PROD. OP FIELD

$565,3 \subseteq 8$

CIL-PRD E/RD

GAS-PRD

$\begin{array}{rr}191 & 0.5 \\ 635 & 1.7 \\ 2231 & 3.3 \\ 3886 & 3.9 \\ 7273 & 4.3 \\ 10720 & 4.4\end{array}$

11

12

15
21

307

$10720 \quad 4.4$

COONTY

FON NELS

\begin{tabular}{|c|c|c|}
\hline & $N O M-R$ & REL I \\
\hline IEAR & FLOW & LI FI \\
\hline $\begin{array}{l}1970 \\
1969 \\
1968 \\
1967 \\
1966\end{array}$ & $\begin{array}{l}0.0 \\
0.0 \\
0.0 \\
0.0 \\
0.0\end{array}$ & $\begin{array}{l}2 \\
2 \\
1\end{array}$ \\
\hline
\end{tabular}

$\begin{array}{rrr}\text { OII-PRD } & \text { E/WD } & \text { GAS-PRD } \\ & & \\ 401 & 0.4 & 246 \\ 1917 & 2.0 & 1143 \\ 2350 & 3.2 & 1303 \\ 5341 & 7.3 & 2700 \\ 8468 & 23.2 & 0\end{array}$


ABANDONED ONSHORE OIL FIELDS WITH OVER 250,000 BBL. COMOLATIVE PRODUCTICN

DST DISC ABD. PLD NDY PIELD NAME

O7C $19501974 \quad 81304665$ SAXON (SERRATT)

DST DISC ABD. FLD NOM FIELD NAME

07. 195219676348666 BAYS (GOEN LIME)

\begin{tabular}{|c|c|c|c|}
\hline DE P TH & GRAVITY & \multicolumn{2}{|c|}{$\mathrm{CU} \mathrm{MO} \mathrm{L}$. } \\
\hline 2,010 & 42.0 & & \\
\hline & & NOM-W & EL IS \\
\hline YEAR & & PIOW & L I PT \\
\hline 1974 & & 0.0 & 0.8 \\
\hline 1973 & & 0.0 & 1.8 \\
\hline .1972 & & 0.0 & 2.0 \\
\hline 1971 & & 0.0 & 2.. 6 \\
\hline 1970 & & 0.0 & 3.0 \\
\hline 1969 & & 0.0 & 3.. 0 \\
\hline $196 ?$ & & 0.0 & 3.0 \\
\hline 1966 & & 0. & 2 \\
\hline
\end{tabular}

ROD. OF FIELD

539,822

COJNTY

RUNNELS

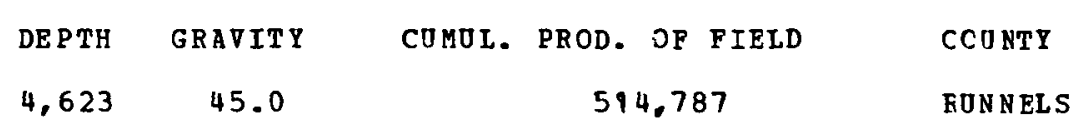

YEAP

NUM-RELIS

OIL-PRD E/HD

GAS-PRD

1966

$0.0 \quad 1.7$

3139

5.2 
ABANDONED ONSHORE OII FIELDS RITH OVER 250,000 BBL. COAOLATIVE PRODOCT ION

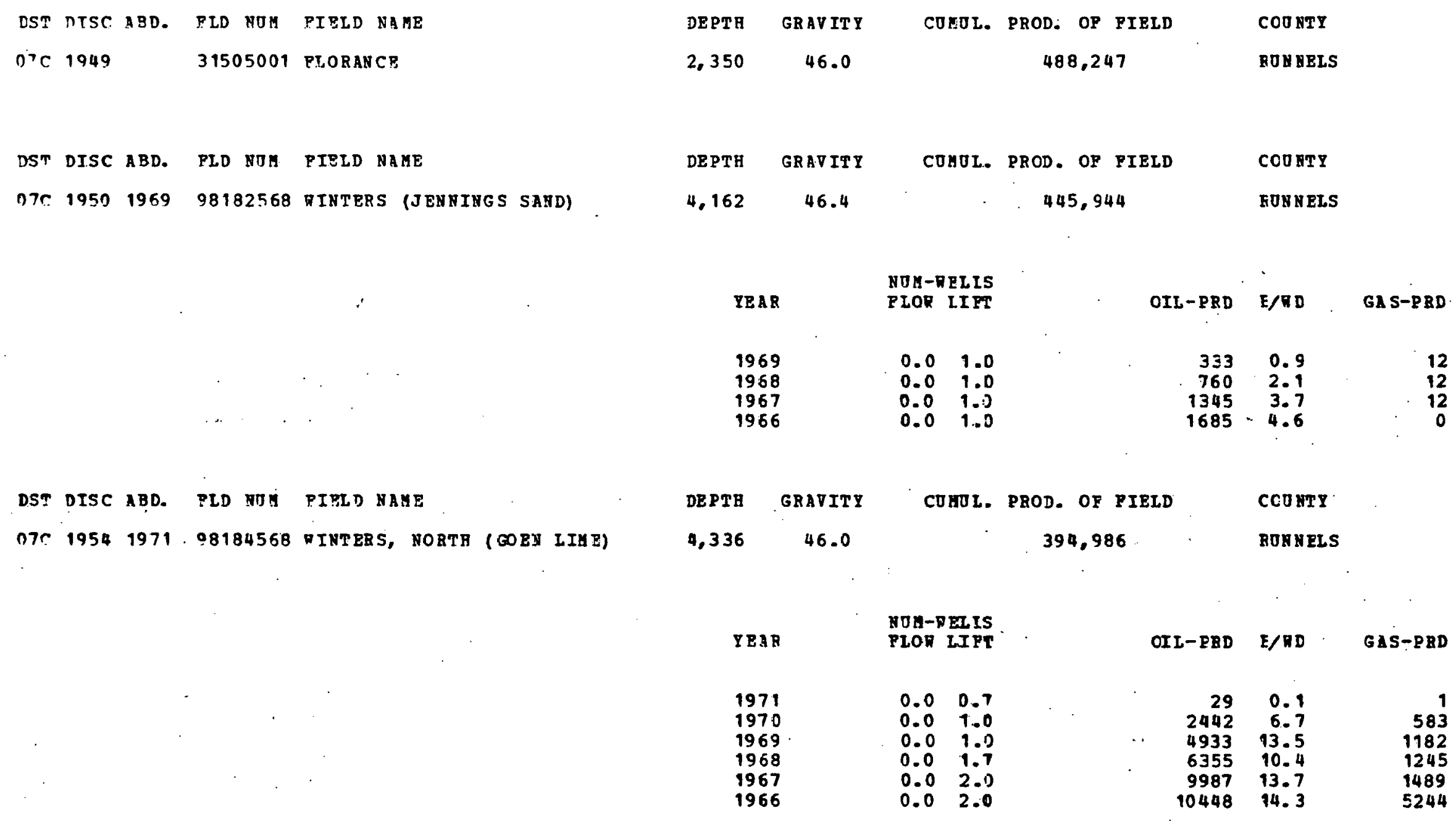


ABANDONED ONSHORE OIL FIELDS VITH OVER 250,000 BPL. COMOLATIVE PRODUCTICN

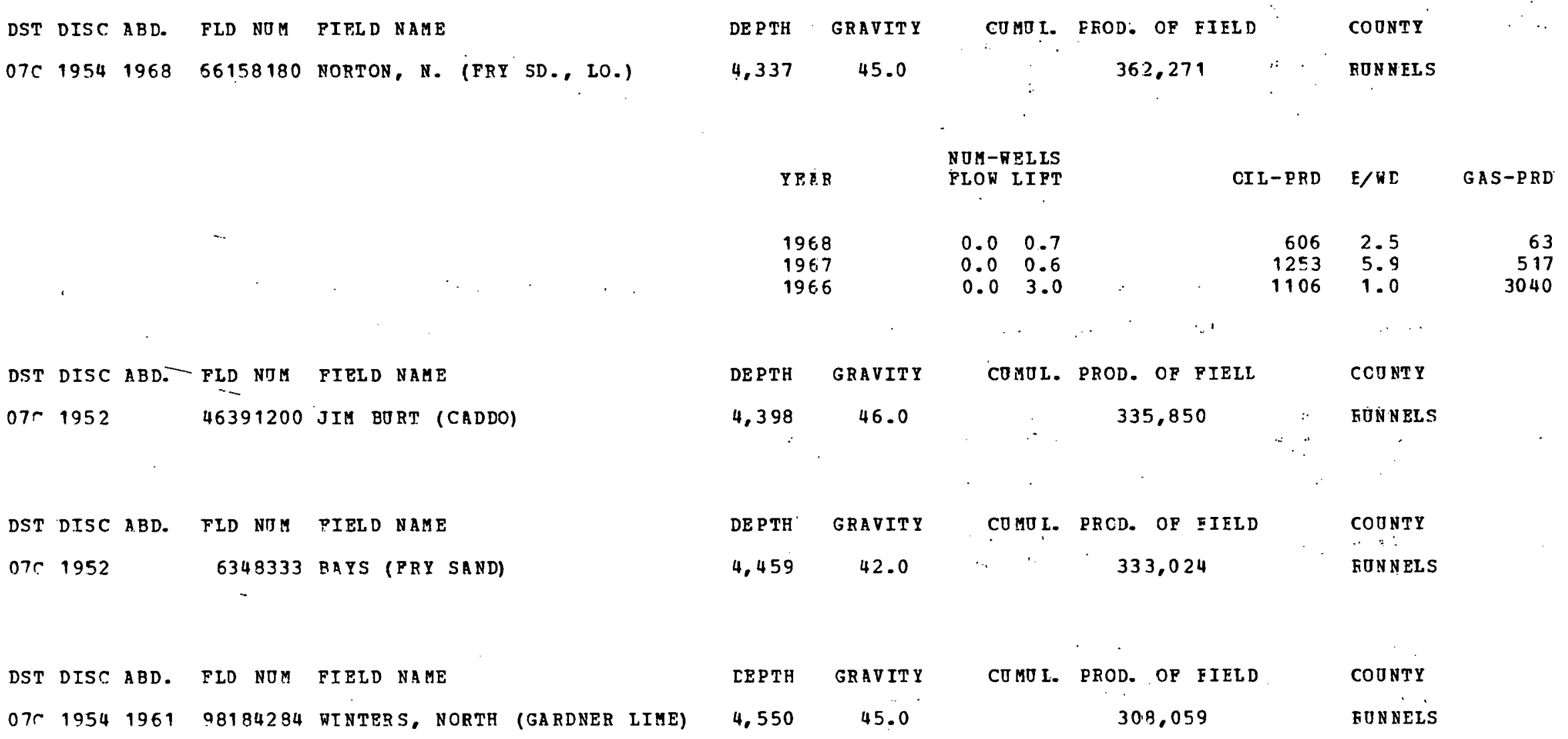


ABANDONED ONSHORE OEL EIEIDS NTH OVER 250,000 BBL. COMULATIVE PRODOCTICN

DST DISC ABD. PLD NUM FIELD NAME

O7م $19551974 \quad 32011852$ FORT CHADBOURNE (MORRIS SD.)

\begin{tabular}{|c|c|c|c|}
\hline DEPTH & GRAVITY & \multicolumn{2}{|c|}{ CU } \\
\hline 4,968 & 38.6 & & \\
\hline YEAR & S & $\begin{array}{l}\text { NOM- } \\
\text { FLOR }\end{array}$ & $\begin{array}{l}\text { EL LS } \\
\text { LIFT }\end{array}$ \\
\hline $\begin{array}{l}1974 \\
1973 \\
1972 \\
1971 \\
1970 \\
1969 \\
1968 \\
1967 \\
1966\end{array}$ & $\begin{array}{l}4 \\
2 \\
6 \\
3 \\
3 \\
7 \\
7\end{array}$ & $\begin{array}{l}0.0 \\
0.0 \\
0.0 \\
0.0 \\
0.0 \\
0.0 \\
0.0 \\
0.0 \\
0.0\end{array}$ & $\begin{array}{l}1.2 \\
0.2 \\
1.0 \\
1.0 \\
1.0 \\
1.4 \\
2.0 \\
2.0 \\
1.1\end{array}$ \\
\hline
\end{tabular}

DE PTH

GRAVITY

3,000
43.0
COUUL. PROD. OF PIELD

272,404
COONTY

BONNELS.

O7. 1956196769770666 PAUL THOHAS (3000 SAND)

Y E AR

1967

1966
NOM-VRLIS

$0.0 \quad 1.7$

0.02 .0
303,718

OIL-PRD E/RD

GAS-PRD

$\begin{array}{rrrr}900 & 2.1 & & 7 \\ 49 & 0.8 & & 5 \\ 321 & 0.9 & 9 \\ 2.00 & 0.5 & 7 \\ 1907 & 5.2 & \\ 4213 & 8.1 & 12 \\ 6848 & 9.4 & 246 \\ 10115 & 13.9 & 3243 \\ 9379 & 14.0 & & 7821 \\ & & & 4737\end{array}$

COONTY

FON NELS 
1 BA IDOAED ORSHORE OIL FIELDS ITH OVER 250,000 B8L. COHOLATIVE PBODOCTICH

DST DISC ABD. PLD NTY PIELD NAHE

O7ก $19571966 \quad 62199500$ UITCRELL (PRY SAND)

DST DISC ABD. PLD NUE FIELD MAAE

O7C 1959197263271250 MOTLEY, NR (CANYOM)
DEPTH
GRAVIT Y
COIOL. FROD. OP FIELD
COONTY
4,322
44.4
272,281
RON NELS

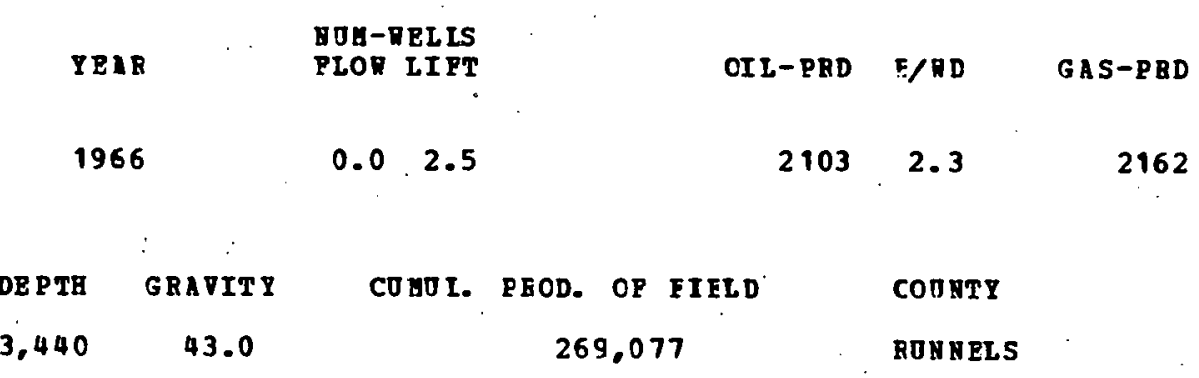

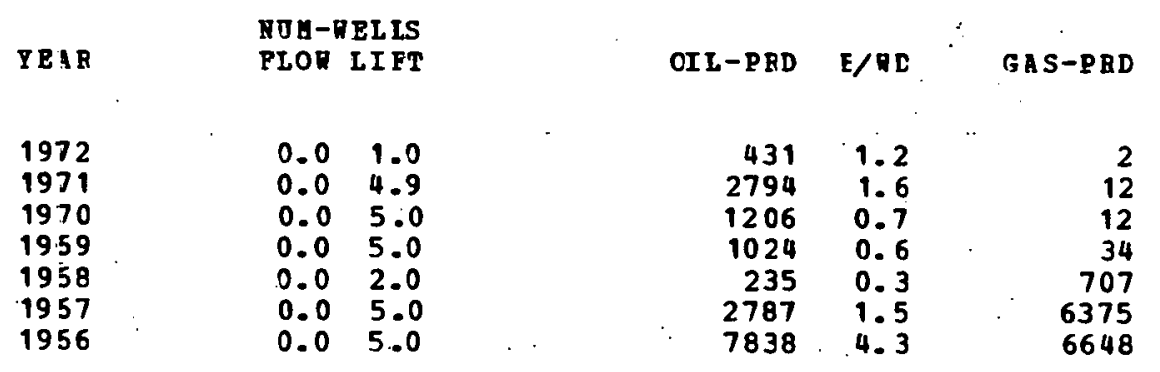

COOHTY : ROHEELS
24 PIELD (S) 
A BANDONED ONSHORE OII RIEIDS WITH OVER 250,000 BBI. ZTMOLATIVE FRODOCTION

DST DISC ABD. FID NTU FIELD NAEE

07C $19531975 \quad 21340500$ SOX-RROWN (STRAWM)

DST DISC ABD. PLD NUA FIELD NAME

07C $19521968 \quad 14010333$ BTTLER (CANYON)

$\begin{array}{ccccl}\text { DEPTH } & \text { GRAVITY } & \text { COMOL. } & \text { PROD. OF FIELD } & \text { COUNTY } \\ 5,550 & 42.0 & & 623,346 & \text { SCHLEICHER }\end{array}$

Y B A R

NOM-RELIS
FLOR LIFT

OIL-PRD E/WD

GAS-P BD

1976

1974

1973

1972

1972

1970

1969

1968

1967

1966

$\begin{array}{ll}0.0 & 1.3 \\ 0.0 & 1.8 \\ 0.0 & 2.3 \\ 0.0 & 3.0 \\ 0.0 & 2 . .7 \\ 0.0 & 2.0 \\ 0.0 & 2.3 \\ 0.0 & 3.8 \\ 0.0 & 4.0 \\ 0.0 & 5.0\end{array}$

$140 \quad 0.3$

19302.

60667.4

$2225 \quad 2.0$

$2843 \quad 2.9$

$\begin{array}{ll}5286 & 7.2 \\ 2392 & 2.9\end{array}$

3.0

$6462 \quad 4.4$

$8527 \quad 4.7$

$$
\begin{array}{r}
8 \\
11 \\
3942 \\
2211 \\
552 \\
15 \\
2068 \\
5462 \\
8972 \\
6799
\end{array}
$$

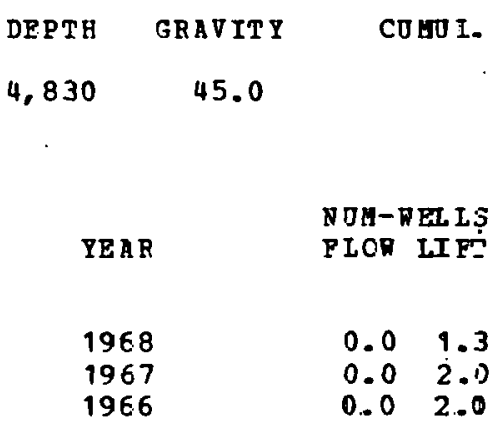

366,645

CODNTY

SCHIEICHER

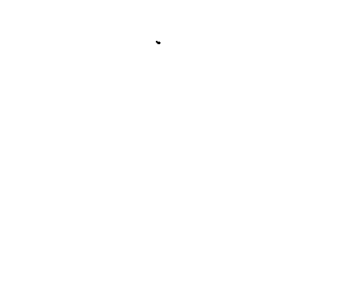

COUN TY : SCHL E ICAEF
2 PIELD (S)

$\begin{array}{rrr}\text { OIL-PRD } & \text { F/HD } & \text { GAS-PED } \\ & & \\ 845 & 1.7 & 1145 \\ 3064 & 4.2 & 9526 \\ 5340 & 7.3 & 146 \mathrm{S1}\end{array}$

989,991 COHJLATIVE PROD. PROM ABAHDONED PIELDS 
ABANDONED ONSHORE OIL PIELDS WITH OVER 250,000 BBL. COBOLAT IVE PRODUCTION

DST NTSC ABD. FLD NT:I PIELD NAYE

07 C 19531968 . 597332 DAMC (DEVONTAM)

DST DISC ABD. FID VOM FIELD NAEE

07C $19531972 \quad 587498$ ADAMC (ELLEN BURGER)

$\begin{array}{rcccc}\text { DEPTH } & \text { GRAVITI } & \text { CUEOL. } & \text { PROD. OF FIELD } & \text { CONNTY } \\ 10.490 & 47.1 & 4.035,160 & \text { OPTOK }\end{array}$

YEAR

ROH-RELIS

OII-PRD E/RD GAS-PBD

1968

1967
1966

$\begin{array}{rr}9.3 & 0.0 \\ 23.1 & 0.0\end{array}$

$\begin{array}{ll}23.1 & 0.0 \\ 24.0 & 0.0\end{array}$

60633017.8

6063317.8

$156555 \quad 18.6$

$186333 \quad 21.3$

536135

1565050

1565188

$\begin{array}{rcccc}\text { DEPTH } & \text { GRAVITY } & \text { CUMOL. } & \text { PROD. OP PIELD } & \text { COOHTY } \\ 11,575 & 53.2 & 962,328 & \text { OPTON }\end{array}$

\begin{tabular}{|c|c|c|}
\hline YEAR & $\begin{array}{l}\text { NOH- } \\
\text { PLON }\end{array}$ & $\begin{array}{l}\text { ELIS } \\
\text { LIFI }\end{array}$ \\
\hline $\begin{array}{l}1972 \\
1971 \\
1970 \\
1969 \\
1968 \\
1967 \\
1966\end{array}$ & $\begin{array}{l}0.0 \\
0.0 \\
0.0 \\
0.0 \\
0.0 \\
0.0 \\
1.5\end{array}$ & $\begin{array}{l}0.5 \\
1.0 \\
1.9 \\
2.0 \\
2.0 \\
2.0 \\
0.05\end{array}$ \\
\hline
\end{tabular}

$\begin{array}{rrr}\text { OrL-PRD } & \text { E/RD } & \text { GAS-PBD } \\ & & \\ 5316 & 29.1 & 1886 \\ 1.2852 & 35.2 & 2158 \\ 14211 & 20.3 & 19142 \\ 8420 & 11.5 & 10791 \\ 18300 & 25.1 & 10984 \\ 32717 & 44.8 & 12033 \\ 25153 & 34.5 & 14683\end{array}$


ABANDOMED ONS 9ORE OIL ZIELDS WITH OVER 250,000 BBL. COHTLATIVE PROLUCTION

DST DISC ABD. FLD NYI FIBLD NABE

DEPTR GRATITY

$07 \mathrm{C} 19621975$

2212222 A.MACKER-TIPPETT, S. (BEN), EP.) 9,590

45.0
YEAB

1975

1974

1973

1972

1971

1970

1969

1968

1967

1966

DST DLSC ABD. FLD NOY FIELD NABE

07r 19511962.31236333 FLAT ROCK (ELLENBDRGER)

DST DISC ABD. PLD NO PIBLD NAGE

DEPTH GRAVITI

07C 1961197518105900 CHRISTOVAL (STRADE 5100, LORER) 5, 156

\section{DE PTH}

10,726

GRAVIT Y

60.2

(3)

39.0
COHJL. PROD. OP PIELD

732.255

$\operatorname{cov} \operatorname{sit}$

OFTCR

$\begin{array}{ll}\text { MOH-WELLS } \\ \text { PLOH LIFT } \\ \\ 0.0 & 0.2 \\ 0.7 & 0.0 \\ 1.0 & 0.0 \\ 1.0 & 0.0 \\ 1.0 & 0.0 \\ 1.0 & 0.0 \\ 0.1 & 0.9 \\ 1.2 & 1.9 \\ 2.0 & 3.0 \\ 9.0 & 2.4\end{array}$

COARL. PROD. OP PIBLD

COONTI

524,306

CP.TON

CU घUT. RBOD. OF EIELD

CODNTY

416,564

oprox

Y EAR
1975
1974
1973
1972
1971
1970
1969
1968
1967
1966

HOM-DELIS

PLOD LITT

$\begin{array}{ll}0.0 & 0.9 \\ 0.0 & 0.8\end{array}$

0.01 .0

0.01 .0

$0.02 .-2$

$0.0 \quad 3.0$

0.03 .0

0.03 .0

$0.4 \quad 2.6$

1.91 .1

$\begin{array}{rrr}\text { OIL-PRD } & \text { E/ GD } & \text { GAS-PRD } \\ & & \\ 2902 & 8.7 & 4349 \\ 2690 & 9.8 & 1547 \\ 3461 & 9.5 & 2638 \\ 7647 & 21.0 & 7369 \\ 5643 & 7.1 & 9710 \\ 8368 & 7.6 & 6813 \\ 8546 & 7.8 & 10682 \\ 20726 & 18.9 & 19118 \\ 27852 & 25.4 & 26565 \\ 39331 & 35.9 & 47085\end{array}$

GA S-PRD

1412
10564

10564

17653
43629

83246

108404

15149

22231

87143

173209 
A BA NDONED ONSHORE OIL PIELDS NTT OVER 250,000 BBL. COMULATIPE ERODUCTICA

DST DISC ABD. PLD NOM FIELD NAME.

07r 1963197349418600 KI Rg nOdNTAIN, S. (8200)

DST DISC ABD. FLD NUY FIELD HAHE

07C $19541967 \quad 89134500$ TEXEL (ELLENBURGER)

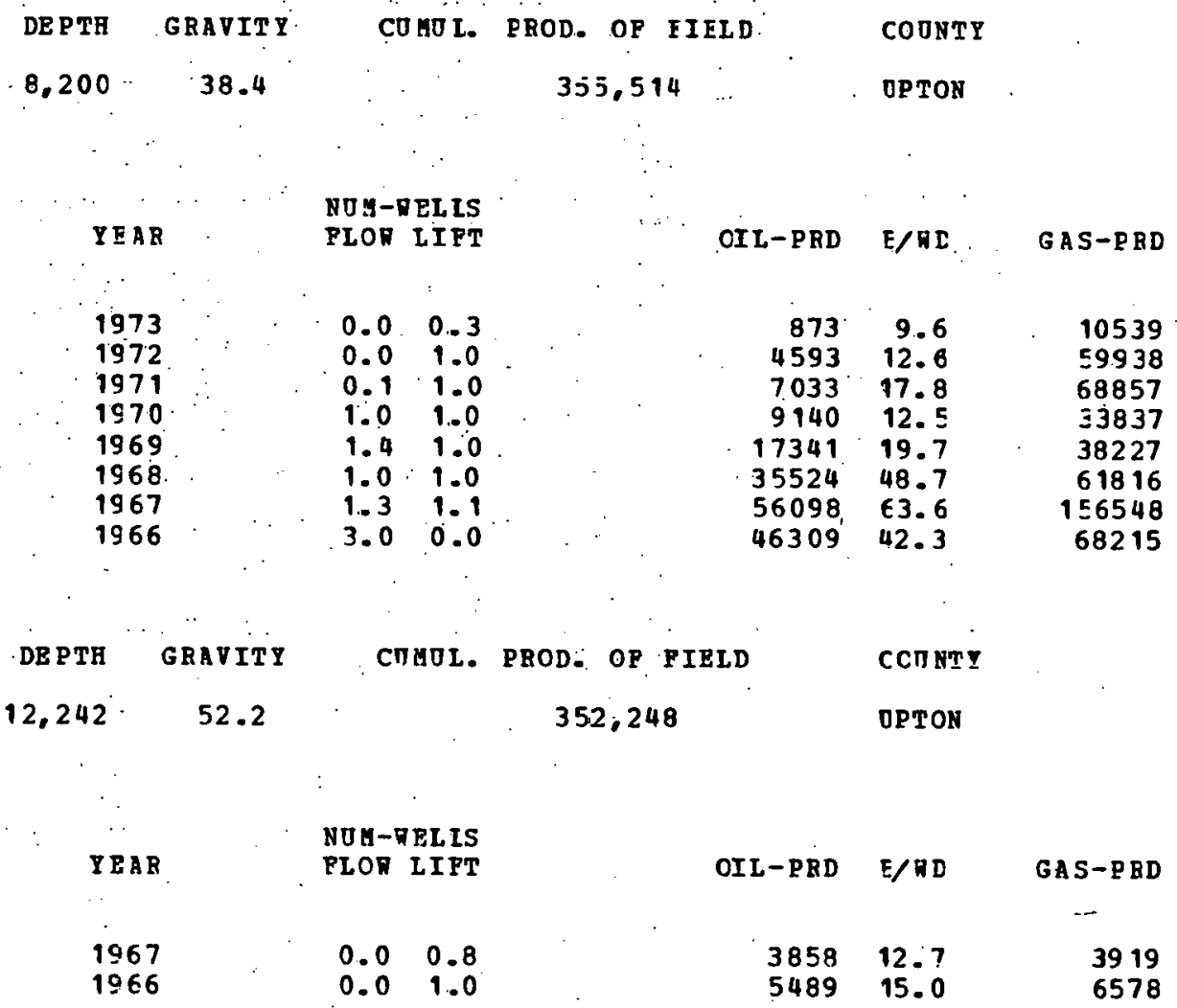


ARANDONED ORSHORE CII PIELDS VITH OVER 250,000 REL. COMOLATIVE PRODOCTICN

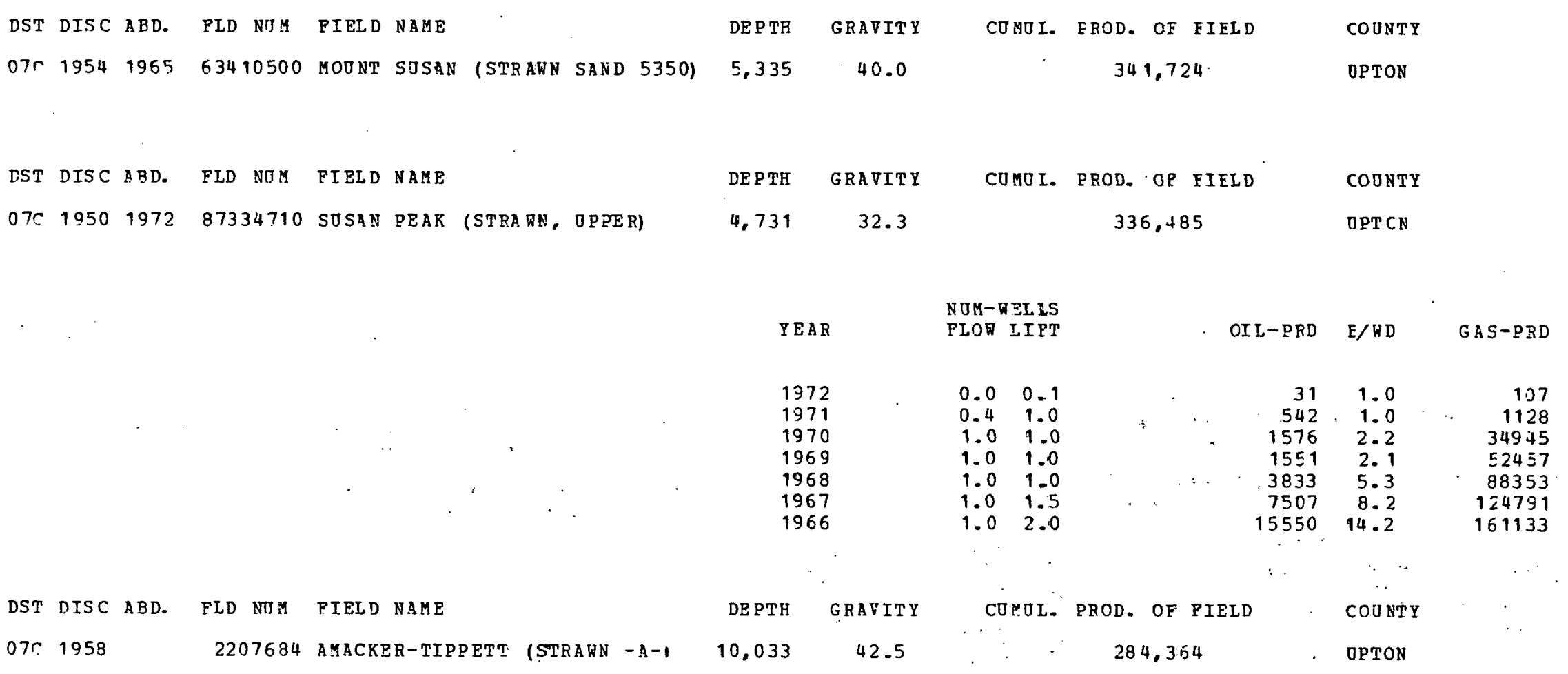


ABANDONFD ONSHORE OIL FIELDS DITH OVER $250,000^{\circ}$ BBL. COMOLAT IVE PRODOCT ION

DST DISC ARD. PID NOM PIRLD NAHE

O?C $19541972 \quad 4310200$ ATRICE (BARKET SAND)

$\begin{array}{cc}\text { DEPTE } & \text { GRAVITY } \\ 4,676 & 35.4\end{array}$

TEAR

1972

1971

1970

1969

1968

1967

1966

COONTY : OPTOR

DISTRICT $07 \mathrm{C}$
11. PIELD (S)

45 PIELDS
COAOL. PROD. OP PIELD

271.791

CODNTI

EFTCR
ROH-RELLS PIOT LI PT

$0.0 \quad 0.7$

$0.0 \quad 3.0$

0.03 .0

0.07 .0

0.07 .5

0.07 .3

3.04 .0

$\begin{array}{rrr}\text { OIL-PAD } & \text { E/ND } & \text { GAS-PBD } \\ & & \\ 350 & 1.4 & 2739 \\ 1.096 & 1.0 & 19407 \\ 2051 & 1.9 & 43355 \\ 6747 & 2.6 & 40933 \\ 7927 & 2.9 & 39952 \\ 10600 & 4.0 & 55967 \\ 14212 & 5.6 & 38425\end{array}$

8,612,749 CUHDLATIVE PROD. FROA ABALDONBD TIBLDS

38,177,878 COHOLATIVE PRCD. PROA ABAHCOREL FIELDS 
ABA NDONED CNSHORE OIL FIZLDS ITH OVER 250,000 EBI. COMOLATIVE PRODOCTICA

NST DISC ABD. PLD YYM PIPLD NAME

08

195619715166555 BARKE (PENN.)

\begin{tabular}{|c|c|c|}
\hline DEPTH & GRAVITY & COHJL. \\
\hline 8,956 & 43.1 & \\
\hline IEAR & & $\begin{array}{l}\text { NOM-RELIS } \\
\text { FLOR LIFT }\end{array}$ \\
\hline $\begin{array}{l}1971 \\
1970 \\
1969 \\
1968 \\
1967 \\
1966\end{array}$ & & $\begin{array}{rr}0.0 & 2.6 \\
0.1 & 28.7 \\
0.8 & 72.6 \\
0.0 & 65.5 \\
0.0 & 69.2 \\
0.8110 .1\end{array}$ \\
\hline
\end{tabular}

8

CCONTY

$11,910,058$

ANDEENS

$\begin{array}{rccc}\text { DEPTH } & \text { GRAVITY } & \text { CJMOL. PHOD. OF PIELD } & \text { CCOHTY } \\ 11,000 & 40.5 & 1,260,503 & \text { AN D RERS }\end{array}$

DST DISC ABD. FLD NOM FIBLD NAME

I A A R

NOH-9ELIS

OIL-PRD

E/T D

GA S-P BD

23332.5

15.8

$292727 \quad 10.9$

49923420.9

87888534.8

106542026.3

20319

472118

344433

983909

$17<1083$

2898814

OA 1949197325189600 DOLLARRIDE, BAST (SILURIAE)

$\begin{array}{ll}0.0 & 0.2 \\ 0.0 & 1.3 \\ 0.0 & 2.4 \\ 0.0 & 2.0 \\ 1.7 & 1.2 \\ 4.0 & 0.8 \\ 6.0 & 1.0 \\ 2.3 & 6.03\end{array}$


ABA NDONED ONSHORE OIL PIELDS NITA OVRR 250,000 BBL. COMULATIVE PRODUCTICN

\begin{tabular}{|c|c|c|c|}
\hline DE P TE & GRAVIT $Y$ & \multicolumn{2}{|c|}{ CO מס I. } \\
\hline 8,120 & 43.8 & & \\
\hline YEAR & & $\begin{array}{l}\text { NUG- } \\
\text { FLOR }\end{array}$ & $\begin{array}{l}\text { ELIS } \\
\text { LIFT }\end{array}$ \\
\hline $\begin{array}{l}1973 \\
1972 \\
1971 \\
1970 \\
1968 \\
1967 \\
1966\end{array}$ & & $\begin{array}{l}0.0 \\
0.0 \\
0.0 \\
0.0 \\
0.0 \\
0.0 \\
0.0\end{array}$ & $\begin{array}{l}0.1 \\
0.4 \\
1.0 \\
0.8 \\
0.5 \\
1.0 \\
1.8\end{array}$ \\
\hline
\end{tabular}

932,679

COONTI

AN DREMS
DST OTSC ABD. PLD NOH PIELD NALE

$0819471973 \quad 33232500$ FILLERTON, NOSTH (DEVONIAN)

DST DISC ABD. FLD NOM FIELD NABE

$081942 \quad 58085001$ MASCHо

DST DISC ABD. FLO NTY FIELD NAHE

$0819591973 \quad 8976500$ BLOCK A-41 (ELIENBORGER) $\begin{aligned} & \text { YEAB } \\ & 1973 \\ & 1972 \\ & 1971 \\ & 1970 \\ & 1968 \\ & 1967 \\ & 1966\end{aligned}$

DEPTH GRAVITY

4,615

32.0

DEPTH

11.707

GRAV ITY

45.2
COMUL. PROD. OP FIELD

543.549

CUHOL. PROD. OP FIELD

524,478

$\begin{array}{rrr}\text { CIL-PRD } & \text { E/RD } & \text { GAS-PED } \\ & & \\ 477 & 15.7 & 1 \\ 1029 & 6.8 & 1210 \\ 8447 & 23.1 & 3531 \\ 6668 & 21.9 & 7852 \\ 139 & 0.8 & 150 \\ 2848 & 7.8 & 16077 \\ 4150 & 6.2 & 26682\end{array}$

COONTY

ANDEERS

CCOATY

AND BERS

\begin{tabular}{lll} 
& \multicolumn{2}{c}{ NOM RELIS } \\
YEAR & FLON LI FT \\
& & \\
1973 & 0.0 & 0.2 \\
1972 & 0.0 & 1.0 \\
1971 & 0.0 & 1.0 \\
1970 & 0.0 & 1.0 \\
1969 & 0.0 & 1.0 \\
1968 & 0.0 & 1.0 \\
1967 & 0.0 & 1.8 \\
1966 & 0.0 & 2.0
\end{tabular}

GA S-P RD

2
468
1494
343
12
12
19
0


ABANDONED ONSHORE OIL FIELDS RITH OVER 250,000 BBL. COMULAT IVE RRODUCTICN

DST DISC ABD. FID NIM EIELD NAME

081961196961118664 MIDLAND PARMS (STRAWN)

DST DISC ABD. FLD NDM FIELD NAME

08

$\begin{array}{ccccc}\text { DEPTH } & \text { GRAVITY } & \text { COMOL. } & \text { PROD. OF EIELD } & \text { CCUNTY } \\ 9,690 & 43.6 & & 353.440 & \text { ANDEERS }\end{array}$

YEAR

NOH-NELLS
PIOW LETT

OIL-PRD E/WD

GA S-PRD

1969

1968

1967

1966

$$
\begin{array}{ll}
0.0 & 2.8 \\
0.0 & 4.0 \\
0.0 & 0.8 \\
0.0 & 4.0
\end{array}
$$

12910

1496310.

1886762.

$33443 \quad 22.9$

88385

124105

145215

120037

$\begin{array}{ccccc}\text { DEPTH } & \text { GRAVITY } & \text { CDMOL. } & \text { PROD. OF PIELD } & \text { COUNTY } \\ 9,669 & 50.1 & & 339,296 & \text { ANDREWS }\end{array}$

YEAR

NOH-TELLS

PLOR LIPT

OI L-PRD

GAS-PRD

$\begin{array}{lll}1970 & 0.9 & 0.0 \\ 1969 & 1.0 & C .0 \\ 1968 & 1.0 & 0.0 \\ 1967 & 0.3 & 0.0 \\ 1966 & 1.0 & 1.03\end{array}$

$\begin{array}{rrrr}12014 & 35.9 & & 4948 \\ 15883 & 43.5 & & 22636 \\ 8136 & 22.3 & & 3972 \\ 9283 & 101.7 & & 8556 \\ 7111 & 15.6 & & 12073\end{array}$


A AANDONED ONSHORE OIL FIELDS NITH OVER 250,000 BBL. CUMOLAT IVE PRODOCTION

DST DISC ASD. FID NOM . FIELD NAME

D8 $\quad 1956 \quad 1974 \quad 30394625$ FASKEN (PENN. 8800)

DST DISC ABD. FLD NOV FISLD NAME

$0819591971 \quad 2725250$ ANDRENS (ELLFNBURGER)

DEPTH GR
8,776
YEAR
1974
1973
1972
1970
1969
1968
1967
1966

DE PTH

GRAVIT Y

12,516

46.0

\section{NOM-VELLS}

. II

$\begin{array}{ll}0.0 & 0.1 \\ 0.0 & 0.8 \\ 0.0 & 0.6 \\ 0.0 & 0.8 \\ 0.0 & 1.0 \\ 0.0 & 1.0 \\ 0.0 & 1.0 \\ 0.0 & 2.6\end{array}$

$\begin{array}{ll}0.0 & 0.1 \\ 0.0 & 0.8\end{array}$

$\begin{array}{ll}0.0 & 0.6\end{array}$

.00 .8

010

$0.0 \quad 2.6$

COMUL. PROD. OF FIELD

334,396

OIL-PRD E/VI

$\begin{array}{rr}1 & 0.0 \\ 896 & 2.9 \\ 1657 & 7.8 \\ 610 & 2.0 \\ 3903 & 10.7 \\ 4872 & 13.3 \\ 6450 & 17.7 \\ 12021 & 12.7\end{array}$

0.0
2.9
7.8
2.0
10.7
3.3
7.7
2.7

CCONTY

AN DRERS
GA S-PRD

73
2717
2227
2180
4785
7819
16597
12259

YEAR
1971
1970
1969
1968
1967
1966

NTM-VELIS

PLOW LIPT

$0.0 \quad 1.4$

$0.0 \quad 1.4$

0.02 .0

$0.0 \quad 2: 0$

$0.0 \quad 0.7$

$0.0 \quad 2.0$

$\begin{array}{rr}\text { OII-FRD } & E / H \\ & \\ 4643 & 9 . \\ 6773 & 9 . \\ 9735 & 13 . \\ 11050 & 15 . \\ 13510 & 55 . \\ 15502 & 21 .\end{array}$

GAS-PRD

26725

15181

13013

10985

47816 
ABANDONED OASRORE OIL FIELDS RITH OVER 250,000 BBL. COUULATIVE PRODOCTICN

DST DISC ABD: FLD NOM PIBLD NAME

08195819699202498 BLOCK 9 (PUSSELKAN)

DEPTH
11,683

YBA

1969
1968
1967
1966

DST DISC ABD. PLD NUT PIBLD NAEE

OB 1959196869193952 PARKER (MOLFCA

$\begin{array}{cc}\text { DEPTE } & \text { GRAVITY } \\ 8,895 & 36.0\end{array}$

YE AR

1968
1967

1966
1. F IEID (S)

\begin{tabular}{|c|c|c|c|}
\hline RAVIT Y & CO HO L. & FROD, OP FIELD & COONTY \\
\hline 45.6 & & 326,001 & $\mathbf{S}$ \\
\hline
\end{tabular}

\begin{tabular}{|c|c|c|c|c|c|}
\hline $\begin{array}{l}\text { NOM- } \\
\text { PLON }\end{array}$ & $\begin{array}{l}\text { ELLS } \\
\text { II?T }\end{array}$ & & OIL-PRD & $E / R D$ & GAS-PRD \\
\hline $\begin{array}{l}0.0 \\
0.0 \\
0.0 \\
0.0\end{array}$ & $\begin{array}{l}0.8 \\
1.0 \\
1.0 \\
1.0\end{array}$ & & $\begin{array}{l}3675 \\
6252 \\
6899 \\
9469\end{array}$ & $\begin{array}{l}13.4 \\
17.1 \\
18.9 \\
25.9\end{array}$ & $\begin{array}{r}2101 \\
28595 \\
75949 \\
\equiv 4633\end{array}$ \\
\hline
\end{tabular}

COTHTY : AN DRERS

\begin{tabular}{|c|c|c|c|c|}
\hline $\begin{array}{l}\text { NOL-D } \\
\text { PLOW }\end{array}$ & $\begin{array}{l}\text { EL LS } \\
\text { LI FT }\end{array}$ & OIL -PRD & E/RC & GAS-PRD \\
\hline $\begin{array}{l}0.0 \\
0.0 \\
0.0\end{array}$ & $\begin{array}{l}1.3 \\
4.3 \\
4.0\end{array}$ & $\begin{array}{r}2008 \\
9377 \\
25679\end{array}$ & $\begin{array}{r}4.4 \\
6.4 \\
17.6\end{array}$ & $\begin{array}{r}2543 \\
24347 \\
60086\end{array}$ \\
\hline
\end{tabular}

COONTY

ANDEE HS 
ABANDONED ONSHORE OIL PIELDS NITH OVER 250,000 BBL. COMOLATIVE PRODUCT ION

DST DISC ABD. FLD NIH FIELD NAHE

081954196626544500 DONE, SOOTHEAST (SAN ANDRES)

1

DST DISC $\triangle B D$. PLD NTM PIBLD NAME

OB $19551968 \quad 94482125$ VADDELL (DEVCNIAM)

\section{DE P TH \\ GRAVIT}

3,520

32.1

COMOL. PROD. OP FIELD

764.700

COUNT

CRANE

\begin{tabular}{|c|c|c|c|c|}
\hline YEAR & $\begin{array}{l}\text { NOL-RELIS } \\
\text { PLOR LIPT }\end{array}$ & OIL-PRD & $E / R D$ & GAS-PRD \\
\hline & $0.0 \quad 0.5$ & 514 & 2.8 & 2629 \\
\hline
\end{tabular}

DE PTH GRAVIT

9,868

43.0

CD MO I. PROD. OP FIELD

479,670

COONTY

CRANE

\begin{tabular}{|c|c|c|c|c|}
\hline TEAB & $\begin{array}{l}\text { NOH-WELLS } \\
\text { PLOR LIFT }\end{array}$ & OIL-PRD & $E / R D$ & GAS-PRD \\
\hline $\begin{array}{l}1968 \\
1967 \\
1966\end{array}$ & $\begin{array}{ll}0.5 & 0.5 \\
1.0 & 1.0 \\
1.0 & 4.0\end{array}$ & $\begin{array}{r}2466 \\
14347 \\
15994\end{array}$ & $\begin{array}{r}6.8 \\
19.7 \\
8.8\end{array}$ & $\begin{array}{r}3589 \\
21073 \\
18211\end{array}$ \\
\hline
\end{tabular}


A BANDONED ONSHORE OIL FIELDS NITA OVER 250,000 BBE. COHOLATIVE PRODUCTION

DST DISC ABD. PLD NOM PIELD HAME

$08 \quad 1956197327775332$ RDMARDS -04- (CONNELL SE.)

\section{DEPTH \\ GR AV IT Y}

11.785

47.1

COMDL. PROD. OP FIELD

379,423

COUNTY

CRANE

\begin{tabular}{|c|c|c|c|c|}
\hline IEAR & $\begin{array}{l}\text { NOH-WELIS } \\
\text { PION LIIFT }\end{array}$ & OIL-PBD & $E /$ T [ & GA S-PRD \\
\hline $\begin{array}{l}1973 \\
1972 \\
1971 \\
1970 \\
1969 \\
1968 \\
1967 \\
1966\end{array}$ & $\begin{array}{ll}0.0 & 1.3 \\
0.0 & 1.0 \\
0.0 & 1.0 \\
0.0 & 1.0 \\
0.0 & 1.0 \\
0.0 & .0 \\
0.0 & 1.0 \\
0.0 & 1.9\end{array}$ & $\begin{array}{r}230 \\
4975 \\
12580 \\
12557 \\
11681 \\
12128 \\
11373 \\
12766\end{array}$ & $\begin{array}{r}2.5 \\
13.6 \\
34.5 \\
34.4 \\
32.0 \\
33.2 \\
\equiv 1.2 \\
18.2\end{array}$ & $\begin{array}{l}2279 \\
5670 \\
3799 \\
1483 \\
1378 \\
1427 \\
4398 \\
2542\end{array}$ \\
\hline
\end{tabular}

DST DISC ABD. PLD NOH PIELD NAHE

OB 19611970
DEPTH

5,096

Y E A R

1970

1969

1968

1967

1966

\section{GRAVIT Y}

41.0
CUHOL. PROD. OF FIELD

272,508

\section{NOS-RELIS}

PLOR IIFT

$\begin{array}{ll}0.0 & 0.8 \\ 0.7 & 1.4 \\ 1.0 & 2.2 \\ 1.0 & 3.0 \\ 1.0 & 3.0\end{array}$
GAS-PRD

$\begin{array}{rrr}\text { CIL-PRD } & \text { E/RD } & \text { GAS-PRD } \\ & & \\ 512 & 1.7 & 578 \\ 1387 & 1.8 & 1951 \\ 4597 & 4.0 & 4863 \\ 19705 & 13.5 & 12367 \\ 25958 & 17.8 & 17763\end{array}$


A BANDONED ONSHORE OIL FIELDS RITH OVER 250,000 BBL. COUULATIVE PRODUCT ION

DST DTSC ABD. PLD NTI PIELD NAHE

nB $1954 \quad 1973 \quad 52624500$ IEA (ICKEE)

\begin{tabular}{|c|c|c|c|c|}
\hline DEPTE & GR AV IT Y & COEOL. & PROD. OP PIELD & CCORTY \\
\hline 7,490 & 42.5 & & 262.483 & CRA ME \\
\hline
\end{tabular}

RE AR

NOH-BELIS

FLOD LIFT

OIL-PED E/HC

GA S-P RD
1973

1972

1971

1970

1969
1968

1968
1967

1966

0.01 .6

$0.0 \quad 2.0$

0.02 .0

0.02 .0

0.02 .0

0.02 .0

0.02 .0

0.02 .0
5 FIELD (S)

$\begin{array}{rrr}11613 & 20.1 & 20919 \\ 15672 & 21.5 & 14372 \\ 28506 & 39.0 & 9239 \\ 33730 & 46.2 & 8897 \\ 22017 & 30.2 & 6455 \\ 19036 & 26.1 & 8511 \\ 17142 & 23.5 & 12748 \\ 14024 & 19.2 & 7375\end{array}$

COONTY: CRANE
2,158,784 COKOLATIVE PROD. PROA ABANDONEC PIEIDS 
ABA RDONED ONSHORE OIL PIBLDS ITH OVER 250,000 BBL. COUOLATIVE PRODOCTICN

DST DISC ABD. PID NTM PIDLD NAME

OS $19531973 \quad 70537396$ PENRELL (POSSELEAN)

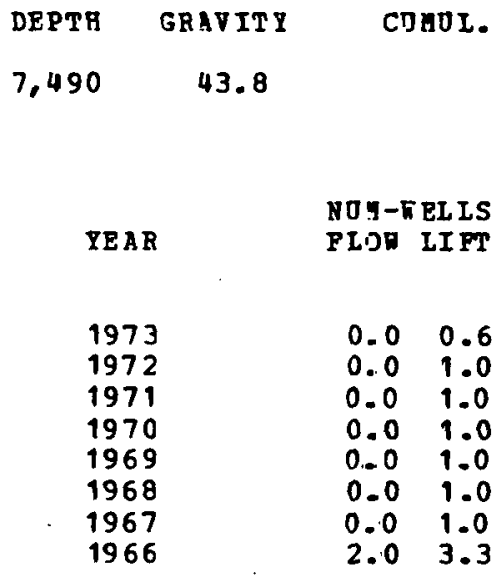

CCOATY

$1,824,087$

ECTCB
DST DISC ABD. FID NUM FIELD NAME

$08 \quad 195019.76 \quad 99275750$ YARBROJGH \& ALIEN (RADDEIL)
DE PTH

10,110
GRAVITY

39.0
TOKOL. PROD. OF PIELD

$1,066,331$
OII-PRD E/RD GAS-PRD

$\begin{array}{rrr}1923 & 9.0 & 5152 \\ 4046 & 11.9 & 6803 \\ 4436 & 12.2 & 2043 \\ 5955 & 16.3 & 1352 \\ 4572 & 12.5 & 587 \\ 3108 & 8.5 & 1465 \\ 3625 & 9.9 & 599 \\ 4640 & 2.4 & 7502\end{array}$

$\cot \operatorname{RTY}$

ECTOR

$\begin{array}{lrrrrr}\text { TEAR } & \begin{array}{r}\text { NOH-REIIS } \\ \text { PIOR IIFT }\end{array} & \text { OII-PRD } & \text { E/RD } & \text { GAS-PRD } \\ & & & & & \\ 1976 & 0.0 & 0.3 & 1363 & 11.2 & 4804 \\ 1975 & 0.0 & 1.0 & 5111 & 14.0 & 20431 \\ 1974 & 0.0 & 1.0 & 5324 & 14.6 & 15065 \\ 1973 & 0.0 & 1.0 & 7657 & 21.0 & 16899 \\ 1972 & 0.0 & 1.9 & 10643 & 15.2 & 43560 \\ 1971 & 0.0 & 2.5 & 21659 & 23.7 & 51629 \\ 1970 & 0.0 & 2.8 & 41121 & 39.8 & 42483 \\ 1969 & 0.2 & 4.8 & 91533 & 50.2 & 90587 \\ 1968 & 0.9 & 6.2 & 123126 & 47.6 & 109335 \\ 1967 & 1.0 & 5.9 & 117302 & 46.5 & 130097 \\ 1966 & 1.0 & 5.0 & 105107 & 48.0 & 99760\end{array}$


ABA NDORED ONSHORE OII PIELDS RITA OVER 250,000 8BL. COHOLATIVE PRODJCTICR

DST DISC ABD. PLD NII PIELD RAHE

$08 \quad 1956 \quad 1976 \quad 88071522$ TXI (PERMSTLVANIAN)

DST DISC ABD. PLD NOA FIBLD NABE

$08 \quad 19531967 \quad 730250$. ADDIS (SAN ANDRES)

$\begin{array}{ccccc}\text { DEPTE } & \text { GRAVITY } & \text { COHOL. PROD. OP EIELD } & \text { COOHTY } \\ 8,450 & 40.0 & & -1: 045,392 & \text { ECTOR }\end{array}$

\begin{tabular}{|c|c|c|}
\hline & \multicolumn{2}{|c|}{ NOH-RELIS } \\
\hline ZEAR & & \\
\hline $\begin{array}{l}1976 \\
1975 \\
1974 \\
1973 \\
1972 \\
1971 \\
1970 \\
1969 \\
1968 \\
1967 \\
1966\end{array}$ & $\begin{array}{l}0.0 \\
0.0 \\
0.0 \\
0.0 \\
0.0 \\
0.0 \\
0.0 \\
0.0 \\
0.0 \\
0.0 \\
0.0\end{array}$ & $\begin{array}{r}2.3 \\
7.0 \\
7.0 \\
7.0 \\
7.2 \\
7.2 \\
8.0 \\
7.0 \\
7.7 \\
10.4 \\
11.0\end{array}$ \\
\hline
\end{tabular}

E/HE

GAS-PED

$\begin{array}{rrr}388 & 0.5 & 5 \\ 1816 & 0.7 & 2819 \\ 1856 & 0.7 & 10042 \\ 5884 & 2.3 & 52993 \\ 13207 & 5.0 & 52500 \\ 19234 & 7.4 & 61902 \\ 18223 & 6.2 & 76622 \\ 17330 & 6.8 & 70451 \\ 16148 & 5.8 & 80967 \\ 28068 & 7.4 & 142080 \\ 48523 & 12.1 & 208777\end{array}$

DE PTE

GRAVITY

CUMUL. PROD. OP FIELD

$\operatorname{cov} \operatorname{NTT}$

4,260

34.4

904,381

ECTOR

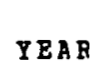

NUM-NELIS

PION IIFT

OIL-PRD E/RD

GAS-PRD

1967

0.02 .0

$\begin{array}{lr}0.0 & 2.0 \\ 0.0 & 24.0\end{array}$

3373
42925

4.6

3088 
ABANDONED ONSHCZE OII FIELDS HITH OVER 250,000 BBL. COMOLATIVE FRODUCTICN

DST DISC ABD. FLD NUM FIELD NAME

OR $19601976 \quad 30398375$ FASKEN, SOOTH (ELLENBURGER)

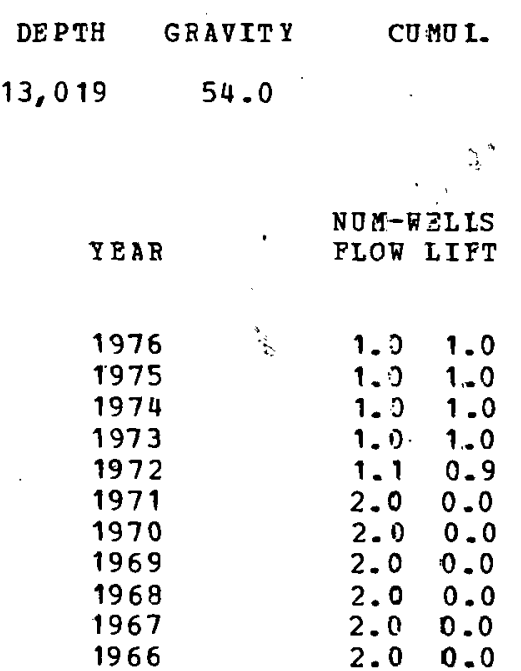

DE PTH

12,130

GRAVITY

51.0

$\begin{array}{rrr}\text { CIL-PRD } & \text { E/RD } & \text { GAS-PBD } \\ & & \\ 23377 & 32.0 & 24194 \\ 29147 & 39.9 & 27484 \\ 32845 & 45.0 & 52174 \\ 31320 & 42.9 & 49711 \\ 25992 & 35.6 & 49847 \\ 26190 & 35.9 & 49984 \\ 34526 & 47.3 & 43582 \\ 30183 & 41.3 & 34459 \\ 29741 & 40.7 & 35483 \\ 29034 & 39.8 & 24171 \\ 30480 & 41.8 & 22356\end{array}$

806,089

COUNTY

ECTOR

DST DISC ABD. PLD NT I FIELD NAME
OB 1963197418254800 CIRCLE BAR (FOSSELMAN)

\begin{tabular}{lll} 
& \multicolumn{2}{l}{ NOH-RELIS } \\
YEAB & PLOW IIFT \\
& & \\
1974 & 0.0 & 0.7 \\
1973 & 0.0 & 2.2 \\
1972 & 0.0 & 3.0 \\
1971 & 0.0 & 3.4 \\
1970 & 0.4 & 3.0 \\
1969 & 1.0 & 4.2 \\
1968 & 1.0 & 5.9 \\
1967 & 1.0 & 2.6 \\
1966 & 1.3 & 5.8
\end{tabular}

E/WD

GAS-PRD

$\begin{array}{rrr}2033 & 7.4 & 4449 \\ 5306 & 6.7 & 14792 \\ 13571 & 12.4 & 28585 \\ 18559 & 14.9 & 54968 \\ 17868 & 14.3 & 39464 \\ 37248 & 19.8 & 95623 \\ 42702 & 16.9 & 93288 \\ 41776 & 31.9 & 60470 \\ 72285 & 28.0 & 112242\end{array}$


ABANDONED ONSHORE OII FIEIDS WITH OVER 250,000 BBL. COUULATIVE PRODUCTION

DST PTSC ARD. PID NTM FIELD NAME

D9 $1962 \quad 1974 \quad 90275125$ YARBRODGH \& ALLEN (CONNEIL)

\section{DEPTE: GRAVITY}

10,317

41.2

YEAR

DST DISC AAD. FLD NUM FIELD NAME

OR $19591970 \quad 2598666$ ANDECTOR, NORTH (WADDELL)

\begin{abstract}
DEPTH GRAVITY
8,085

43.0
\end{abstract}

CUMOL. PROD. OP PIELD

490,644

COUNTY

ECTOR
1974

1973

1972

1970

1969

1969

1968

1967
1966

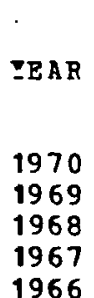

NOL- VELIS

PLOH LIFT

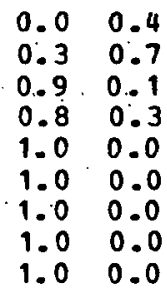

$0.3 \quad 0.7$

$0.9 \cdot 0.1$

.00 .0

$.0 \quad 0.0$

1.00 .0

CUMO I. PROD. OP FIELD

396,622

\section{OIL-PRD E/RI GAS-PRD}

$\begin{array}{lll}2467 \quad 16.2 & 3758\end{array}$

$\begin{array}{rrr}17025 & 50.9 & 25824 \\ 52112 & 142.8 & .27323\end{array}$

$\begin{array}{rrr}52112 & 142.8 & 27323 \\ 58782 & 138.0 & 39538\end{array}$

$60753166.4 \quad 27737$

44016120.6 . 24245

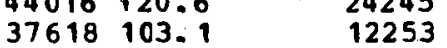

$37618103.1 \quad 12253$

3494793.6
28377850

$\begin{array}{lll}28377 \quad 77.7 & 7149\end{array}$

\begin{tabular}{lll} 
& \multicolumn{2}{c}{ NOH-WELLS } \\
IEAR & FIOV & LIFT \\
& & \\
1970 & 0.4 & 0.3 \\
1969 & 1.8 & 1.0 \\
1968 & 3.0 & 1.0 \\
1967 & 3.0 & 2.7 \\
1966 & 3.0 & 3.0
\end{tabular}

OIL-PRD E/HD GAS-PRD

$672 \quad 2.8 \quad 6300$

$6404 \quad 6.2 \quad 55442$

$7985 \quad 5.5 \quad 84242$

$\begin{array}{rrr}14011 & 6.8 & 120812\end{array}$

$\begin{array}{lll}14780 & 6.8 & 120812\end{array}$ 
ABANDONED CNSHORE OIL FIELDS RITH OVER 250,000 BBL. COMOLATIVE PRODUCTION

DST DISC ABD. FLD NOM PIBLD NAME

$09 \quad 19571976 \quad 30398250$ PASKEN, S. (DEVCNIAN)

\begin{tabular}{|c|c|c|c|}
\hline DE PTE & GRAV IT Y & \multicolumn{2}{|c|}{ cosol. } \\
\hline 11,540 & 44.6 & & \\
\hline YEAR & & $\begin{array}{l}\text { NOM- } \\
\text { PLOR }\end{array}$ & $\begin{array}{l}\text { EEIS } \\
\text { LI FT }\end{array}$ \\
\hline $\begin{array}{l}1976 \\
1975 \\
1974 \\
1973 \\
1972 \\
1971 \\
1970 \\
1969 \\
1968 \\
1967 \\
1966\end{array}$ & & $\begin{array}{l}0.9 \\
1.0 \\
1.0 \\
1.1 \\
1.0 \\
1.0 \\
1.0 \\
1.0 \\
1.0 \\
2.0 \\
2.0\end{array}$ & $\begin{array}{l}0.0 \\
0.0 \\
0.0 \\
0.0 \\
0.0 \\
0.0 \\
1.0 \\
1.0 \\
1.0 \\
0.0 \\
2.0 \\
2.0\end{array}$ \\
\hline
\end{tabular}

DEPTH

SRATITY

B. 650
CONOL. PROD. OP FIELD

359,780
COONTY

ECTCR

DST DISC MD. PLD NTM PIRLD NAEE

(nOLF CAMP -E-)

44.0

TEAR

NUM-TELLS

FLOR IIFT

1972

1971

1970

1969

1968

1967
1966

$\begin{array}{ll}0.0 & 1.0 \\ 0.0 & 9.0 \\ 0.0 & 1.0 \\ 0.0 & 1.0 \\ 0.0 & 1.0 \\ 0.0 & 1.0 \\ 0.0 & 1.0\end{array}$

0.09 .0

$0.0 \cdot 1.0$

0.01 .0

0.01 .0
387,998

$\begin{array}{rrr}\text { OII-PRD } & \text { E/HD } & \text { GAS-PRD } \\ & & \\ 5109 & 15.3 & 20210 \\ 5920 & 16.2 & 19526 \\ 8002 & 21.9 & 3652 \\ 8059 & 20.4 & 3715 \\ 1957 & 5.4 & 8229 \\ 8016 & 17.6 & 32132 \\ 11653 & 16.0 & 31706 \\ 13355 & 18.3 & 34169 \\ 13261 & 18.2 & 38181 \\ 25264 & 23.7 & 87970 \\ 33880 & 23.2 & 129531\end{array}$

COUNTY

ECT CA 
ABA NDONED ONSHORE OIL FIELDS RITH OVER 250,000 BBL. COMOLATIVE PRODUCTICN

DST DISC ABD. FLD NTM FIELD NAME

081966196821289200 CORDEN, NORTH (CISCO)
DE P TH
GRAVITY
CUMOL. PROD. OP FIELD
CONNTY
8,895
42.0
310,033
FCTOR

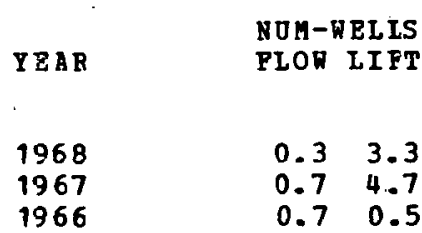

$\begin{array}{cc}\text { DE PTH } & \text { GRAVITY } \\ 8,804 & 40.2\end{array}$

COMOL. PROD. OP PIEID

302,217

$\begin{array}{rr}\text { CIL-PRD } & \text { E/HD } \\ & \\ 90296 & 69.0 \\ 187493 & 54.8\end{array}$

GAS - PRD

43652 95.

94693

DST DISC ABD. PLD NTI PIELD NAHE

$081961 \quad 1970 \quad 50873710$ METZ (RADDELL)

$\operatorname{coONT} \mathrm{Y}$

FCTOR
NOH-NELIS

IEAR

1970

1969

1968

1967

1966

\begin{tabular}{lll} 
& \multicolumn{2}{l}{ NOH-WELIS } \\
TAR & FLON IIFT \\
& & \\
970 & 0.0 & $1 . .2$ \\
969 & 0.0 & 2.0 \\
968 & 0.0 & 2.8 \\
967 & 0.0 & 3.9 \\
966 & 0.0 & 4.0
\end{tabular}

$\begin{array}{rrr}\text { OIL-PRD } & \text { E/HD } & \text { GAS-PBD } \\ & & \\ 6471 & 14.2 & 3511 \\ 12601 & 17.3 & 1798 \\ 14199 & 14.1 & 1298 \\ 33309 & 23.3 & 3132 \\ 39132 & 26.8 & 18207\end{array}$

1298

18207 
ABANDONED CNSHORE OII PIELDS ITR OVER 250,000 BBI. COAOLATIVE PRODUCTICA

DST DISC ABD. PLD NOE FIBLD NAME

O 1960197430399875 . PASREN, SOOTH (ROLPCAEP)

DST DISC ABD. FID WU PIELD NABE

081952.196670537660 PENRELL (NADDELL)

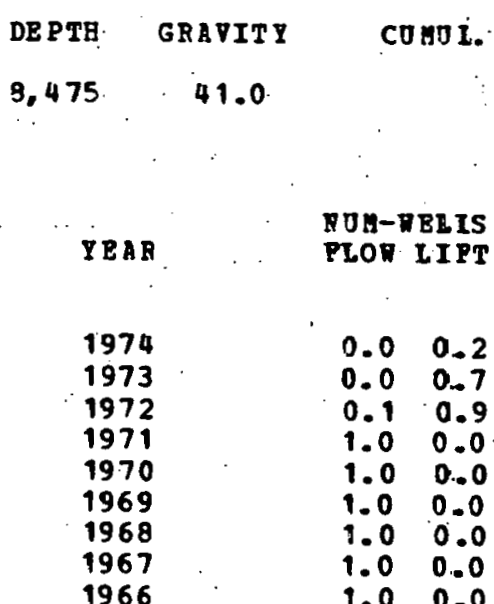

8544

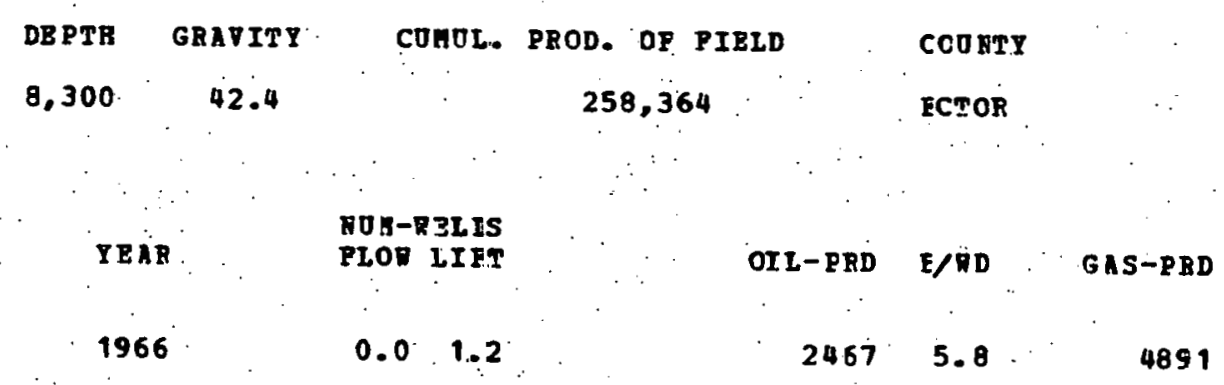


ABANDONED ONSHORE OII FIELDS HITH OVER 250,000 BBL. CUMULATIVE RRODUCTION

DST DISE ARD. FLD NUM FIELD NAME

$08 \quad 1959 \quad 1968 \quad 74793666$ RATLIFF (PENN.)

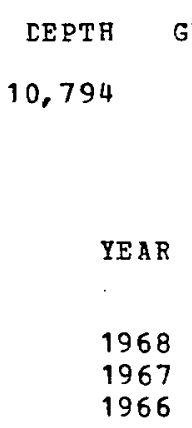

ГEPTH

10,794

YEAR

1968

1967

1966

COJNTY: BCTOF
15 FIELD ( $)$

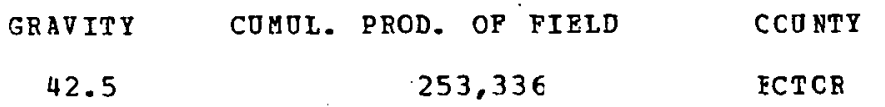

NOM-WELIS FLOW LIFT

\section{OII PRD E/H I}

GA S-P RD

$0.0 \quad 0.8$

$\begin{array}{ll}0.7 & 1.3 \\ 1.8 & 1.3\end{array}$

$797 \quad 2.6$

$7119 \quad 9.8$

$14078 \quad 12.2$

1593

8274

18238

9,372,275 COMULATIVE PROD. PROM ABANDONED FIELDS 
ABANDONED OBSHORE OIL FIEIDS IITH OVER 250,000 BBE. COHOLATIVE PRODOCT IOM

DST DTSC ABD. PID NOA PIELD NAME

$08 \quad 1952$
20704750 CORONE T $(2900)$

COTNTT: HORAR
DEPTH GRAVITY

2,740

19.9

I FIBID (S)
COYOI. PROD. OP FIELD

531,661

CODNTY

HOR ARD

531,661 COLOLATIFE PROD. FROY ABAMDONED PIBLDS 
ABANDONED ORSHORE OIL FIBLDS DITA OVER 250,000 BgL. COMULATIVE PRODUCTICN

DST DISC ABD. PLD YYTM PIRLD MA AE

$08 \quad 1963 \quad 1969 \quad .78865666 \quad$ PDEBEH (POSSELMAM)

$\begin{array}{rcccc}\text { DEPTE } & \text { GRAVITY } & \text { CUNOL. PROD. OP PIELD } & \text { CCORTY } \\ 12,980 & 48.8 & 299,928 & \text { MARTIN }\end{array}$

YEAR

NOE-RELLS

FLOR IIFT

OIL-PRD E/RD

GA S-PRD

1969

1968

1967

$\begin{array}{ll}0.0 & 0.6 \\ 0.0 & 2.0 \\ 0.0 & 2.5 \\ 0.0 & 3.0\end{array}$

$3036 \quad 14.3$

1300917.8

$\begin{array}{ll}17292 & 19.0\end{array}$

351

2172

2568

1966

1 PIELD (S)

299,928 COADLATIE PROD. PROA ABANEOHED PIEIDS 
ARA NDONED ONSHORE OIL FIELDS MITH OVER 250,000 BBL. COHULATIPE PRODOCTICN

DST DISC ABD. PLD NOM FIELD HAME

$08 \quad 1950 \quad 1970$

293750 ABRLL, BAST (RADDELL)

DST DISC ABD. FID NOE FIELD NABE

081949196297889426 T. C. I. (SAN ANDRES)

DST DISC: ABD. PLD NOM PIELD NAME

$08 \quad 1941 \quad 1969 \quad 96373009$ RENTZ

$\begin{array}{ccccc}\text { DEPTH } & \text { GRAVITY } & \text { CUHOL. PROD. OF PIELD } & \text { CCONTY } \\ 6,008 & 44.2 & 321.909 & \text { EECCS }\end{array}$

TEAR

\section{NOM-RELIS}

PLON IIFT

OIL-PRD E/WD

GA S-PRD

1970

1969

1968

1967

$0.0 \quad 0.8$

0.01 .0

$0.0 \quad 1.0$

0.01 .0

321.909

EBC CS

1966

$\begin{array}{ccccc}\text { DEPTH } & \text { GRAVITY } & \text { COAOL. PROD. OP PIEID } & \text { COONTY } \\ 2,200 & 35.1 & 305,662 & \text { EECCS }\end{array}$

DEPTH GRAVITY

COEOL. PROD. OP PIELD

CCORTY

4,400

33.0

305,013

ERCCS

\begin{tabular}{|c|c|}
\hline IEAR & $\begin{array}{l}\text { EOn-D } \\
\text { PLon }\end{array}$ \\
\hline $\begin{array}{r}1969 \\
1968 \\
1967 \\
1966\end{array}$ & $\begin{array}{l}0.0 \\
0.0 \\
0.0 \\
0.0\end{array}$ \\
\hline
\end{tabular}

$\begin{array}{rrr}\text { OII-PRD } & \text { E/HI } & \text { GAS-PRD } \\ & & \\ 771 & 4.2 & 304 \\ 2581 & 7.1 & 797 \\ 2989 & 8.2 & 2563 \\ 3846 & 10.5 & 2153\end{array}$


ABANDONED ONSHORE OIL FIELDS HIT OVER 250,000 BBL. CDGULATIVE PRODUCTION

DST DISC ABD. FLD NOM PIELD NAME

OR $19511969 \quad 40391001$ HENDERSON-PECOS

$\begin{array}{lr}\text { DE PTH } & \text { GRAVITY } \\ 2,040 & 26 . .2\end{array}$

TEAR

1969

1968

1967

COUNTY: PECOS
4 PIELD (S)

\section{COMOL. PROD. OP PIELD CODNTY}

$30.4,045$

ERCCS

$\begin{array}{lrrrrr}\text { MEAR } & \begin{array}{l}\text { NOM-VELIS } \\ \text { FIOR IIFT }\end{array} & \text { OIL-PRD } & \text { E/RI } & \text { GAS-PRD } \\ 1969 & 0.0 & 1.7 & 560 & 0.9 & 5 \\ 1968 & 0.0 & 4.0 & 2349 & 1.6 & 12 \\ 1967 & 0.0 & 6.0 & 4139 & 1.9 & 24 \\ 1966 & 0.0 & 7.5 & 5744 & 2.1 & 0\end{array}$

$1,236,629$ COHOLATIVE PROD. PROA ABANDONED PIEIDS 
AB4 NDONEL ONSHORE JIL PIELDS ITH OPER 250,000 BBL. CUMOLATIVE PRODOCTICA

DS? DTSC ABD. PLO NOM PIELD HAME

D8 $1948 \quad 1972 \quad 25020001$ DIXIBLAND

$\begin{array}{ccccc}\text { DEPTE } & \text { GRAVTT } & \text { CONOL. } & \text { PROD. OP PIELD } & \text { CCOHTY } \\ 3,855 & 40.0 & & 349,714 & \text { FEB }\end{array}$

YEAR

ROA-RELIS PLOD LIFT

OIL-PRD E/RD

GA S-PRD

COJNTY : RERVES
1972

1971

1970

1969

1968

1967

1966

1 PIEID (S)

$$
\begin{array}{ll}
0.0 & 1.0 \\
0.1 & 2.9 \\
0.1 & 5.6 \\
0.0 & 6.0 \\
0.0 & 6.0 \\
0.0 & 6.0 \\
0.0 & 6.0
\end{array}
$$$$
32+\quad 0.9
$$$$
2367 \quad 2.2
$$$$
2563 \quad 1.2
$$$$
2655 \quad 1.2
$$$$
37.90 \quad 1.7
$$$$
\begin{aligned}
& 4134 \cdot 1.9 \\
& 5033
\end{aligned}
$$ 
ABANDONED ONSHORE OIL FIELDS MITH OVER 250,000 BBL. CUMOLAT IVE PRODUCTION

DST DISC ABD. PLD NIM PIELD NAHE

081967197421603500 CREDO, SE. (HCLFCAMP)

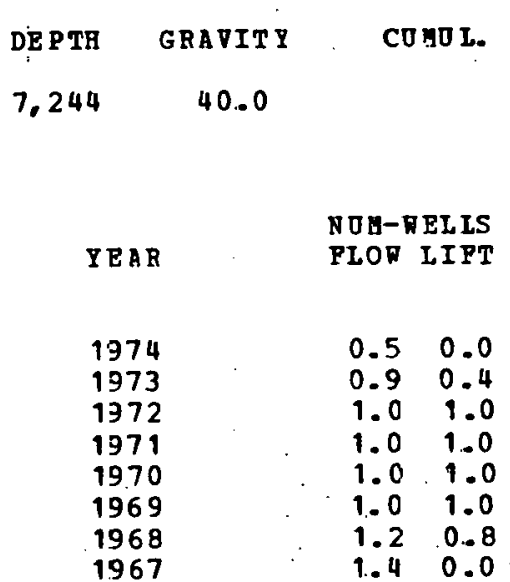

1 PIEID (S)
COONTI

STERLIN G
251,057

$\begin{array}{rrrr}\text { CII-PRD } & \text { E/RD } & \text { GAS-PRD } \\ & & \\ 411 & 2.3 & \\ 1829 & 3.8 & 9472 \\ 5807 & 8.0 & 30949 \\ 15090 & 20.7 & 49320 \\ 18819 & 25.8 & 82646 \\ 45971 & 63.0 & 45617 \\ 86352 & 18.3 & 106073 \\ 76778 & 148.5 & 128232 \\ 73843\end{array}$

251,057 COMOLATIVE PRCD. PROM ABABLONEC PIELDS 
ABANDONED ONSHORE OEL FIELDS WITH OVER 250,000 BEL. CUMOLATIVE ERODUCTION

DST DISC ABD. FLD NTM FIELD NAME

$08 \quad 1942 \quad 1969 \quad ? 3561001$ DYOTE

DST DISC ABD. FLD NUM FIELD NAME.

$08 \quad 19651975 \quad 91364500$ TRIPLP-S (PENN 81725)

\begin{tabular}{|c|c|c|c|}
\hline DE P TH & GRAVITY & \multicolumn{2}{|c|}{ CO } \\
\hline 2,827 & 19.0 & & \\
\hline$Y F A R$ & & $\begin{array}{l}\text { YOM- } \\
\text { PLOB }\end{array}$ & ELIS \\
\hline $\begin{array}{l}1969 \\
1968 \\
1967 \\
1966\end{array}$ & & $\begin{array}{l}0.0 \\
0.0 \\
0.0 \\
0.0\end{array}$ & $\begin{array}{l}0.4 \\
1.0 \\
0.3 \\
1.0\end{array}$ \\
\hline
\end{tabular}

338.486

COUNT

NAR D

$\begin{array}{ccccc}\text { DEPTH } & \text { GRAVITY } & \text { COMUI. FROD. OZ FIELD } & \text { COUNTY } \\ 8.823 & 39.9 & & \ldots 290,312 & \text { HARD }\end{array}$

YEAR
1975
1974
1973
1972
1971
1970
1969
1968
1967
1966

NOM-WEZLS

PIOK LIFT

$\begin{array}{rr}3.3 & 0.4 \\ 8.3 & 0.8 \\ 6.7 & 1.0 \\ 10.0 & 1.0 \\ 0.0 & 3.0 \\ 0.0 & 3.0 \\ 0.0 & 3.0 \\ 0.0 & 3.0 \\ 0.0 & 3.0 \\ 0.2 & 4.6\end{array}$
OII-PRD E/WD GAS-PRD

$\begin{array}{ll}315 & 2.1\end{array}$

$1068 \quad 2.9$

$1304 \quad 14.3$

19895.4

$\triangle \mathrm{AABD}$

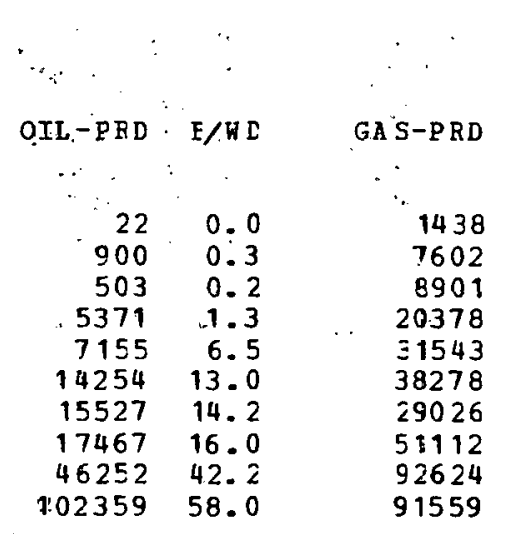


ABANDONED ONSHORE OIL FIELDS RITH. OVER 250,000 BBL. CO EOLAT IVE PRODUCTION

DST DISC ABD. PLD ROA PIELD MAUE

$08 \quad 1965197295138348$ RARD, S. (PBH.))

DEPTE GRAVITY

8.080

43.0

COHOL. PROD. OP TIBID

$255.46 \varepsilon$

\begin{tabular}{rr} 
OIL-PBD & E/RC \\
& \\
3897 & 10.7 \\
2498 & 4.8 \\
7660 & 10.5 \\
14976 & 20.5 \\
29840 & 40.9 \\
69362 & 95.0 \\
118580 & 162.4 \\
\hline
\end{tabular}

MOR-DELIS
PIOD IIFT

$\begin{array}{ll}0.0 & 1.0 \\ 0.0 & 1.4 \\ 0.0 & 2.0 \\ 0.6 & 1.4 \\ 1.0 & 1.0 \\ 1.0 & 1.0 \\ 1.8 & 0.3\end{array}$ coursy.

TARC
GA S-PAD

3 PIBLD (S) 
ABANDONED ONSHORE OIL FIELDS NITH OVER 250,000 BBL. CUMULATIYE PRODUCTION

DST DISC ABD. FLD NOM FIELD NAME

O8 1957196962417450 MONAHANS, NORTH (FUSSELHAM

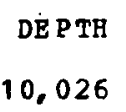

DE P TH

10,026

GRAVITY

37.1
COONTI

DIN KL ER

$\begin{array}{lrrrrr}\text { YEAR } & \begin{array}{r}\text { NOM-AEIIS } \\ \text { PLOR LIPT }\end{array} & \text { CIL-DRD } & \text { E/WD } & \text { GAS-FRD } \\ & & & & & \\ 1969 & 0.0 & 2.7 & 5170 & 5.3 & 11040 \\ 1968 & 0.0 & 4.0 & 51277 & 35.1 & 55011 \\ 1967 & 0.0 & 1.8 & 51707 & 81.0 & 72195 \\ 1966 & 0.1 & 2.9 & 41477 & 37.9 & \$ 1384\end{array}$

DST DISC ABD. FID ROM PIELD NAGE

$\begin{array}{llllll}08 & 1956 & 1972 & 18272333 & \text { CIPCIE } 2 & \text { (DEVONI AH) }\end{array}$

\author{
DEPTH GRAVITY \\ COMOL. PROD. OF FIELD \\ 520,178 \\ COONTY \\ 9,186 \\ 34.5
}

VUM-NELES

YEAR

F LOH LI ?T

1972

1971

1970

1969

1968

1967

1966
$0.0 \quad 2.0$

$0.0 \quad 1.6$

$\begin{array}{lll}0.0 & 1.0 \\ 0.0 & 1.0\end{array}$

0.01 .8

$\begin{array}{ll}0.0 & 3.0 \\ 0.9 & 2.2\end{array}$
983,139

$41477 \quad 37.9$ 
ABANDONRD ONSHORE OIL FIEIDS RITH OVER 250,000 BBL C CUOLATIVE ERODOCTION

DST DISC ABD. FID NTM PIELD NAME

$08 \quad 1964 \quad 1971$

DEPTH GRAVITI

9.233

36.4
CONOL. FROD. OF FIELD

325,288
CODNTY

RINRL RR

YEAR

1971
1970
1969
1968
1967
1966

COONTY: DINRIER

DISTRICT 08
3 PIEID (S)

45 PIEIDS
AS-P BD

\begin{tabular}{|c|c|c|c|c|c|}
\hline $\begin{array}{l}0.0 \\
0.0 \\
0.0 \\
0.0 \\
0.0 \\
0.0\end{array}$ & $\begin{array}{l}0.4 \\
1.9 \\
3.0 \\
3.0 \\
3.0 \\
3.0\end{array}$ & ' & $\begin{array}{r}637 \\
11708 \\
28334 \\
33463 \\
37275 \\
57797\end{array}$ & $\begin{array}{r}4.2 \\
16.7 \\
25.9 \\
30.6 \\
34.0 \\
52.8\end{array}$ & $\begin{array}{r}3049 \\
12195 \\
14313 \\
\vdots 1943 \\
29083 \\
26025\end{array}$ \\
\hline
\end{tabular}

$1,828,605$ COBOL ATIVE PROD. PROA ABANDOHED FI ELDS $34,090,493$ COHOLATIVE PRCD. PROE ABAECORBD PIIIDS 
ARANDONED ONSHOBE OIL FIBIDS NITH OVER 250,000 B9L. COMULATIVE PRODOCT ICY

DST DISC ABD. PID ROA PIELD RAME

O8A 1957.197655573500 LDCY (PENN.)

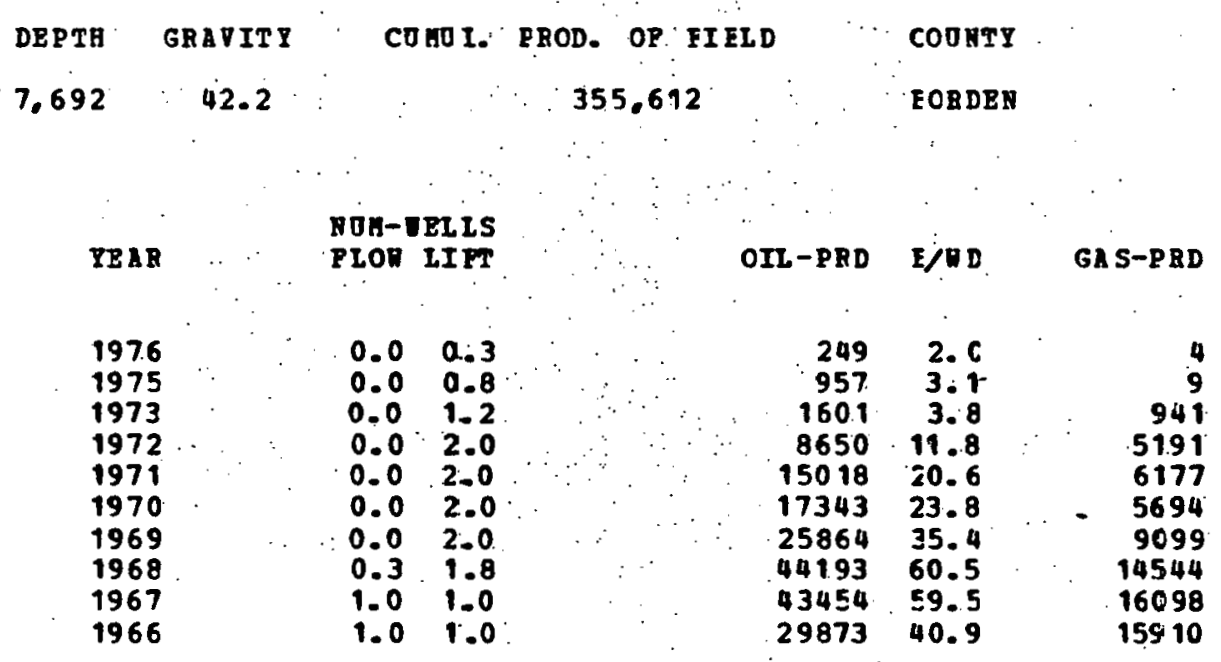

DEPTH GRAUITY

$8,381 \cdot 40.0$

CUBOL. PROD. OF PIBLD

286,472

CContr

EOR LEN

YEAR
1969
1968
1967
1966

MOH-RELLS

PLON II PT

OIL-PRD E/RD

GAS-PRD

0.30 .0

1.90 .0

2.0. 0.0

$2.0 \quad 0.0$

10138.

$\begin{array}{rr}8476 & 12.1 \\ 11997: 16.4\end{array}$

$11997: 16.4$
13539 18.5

3
23
22
0 
APANDONED ONSHORE OII FIELDS VITE OVER 250,000 BBI. COMULATIVE PRODOCTICN

DST DISC ABD. FLD NOM FIELD NAME

089 $19501969 \quad 15359500$ CANING (HOLFCAMP)

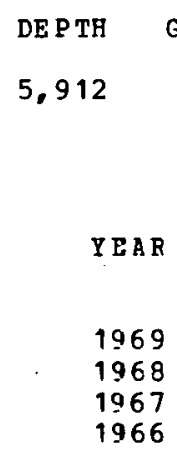

DE P TH

5,912

Y E A R

1969

1968

1967

1966

3 PIELD (S)

\section{GRAVIT Y}

41.0

COMOL. EROD. OF FIEI.D

268.289

COONTY

EOR DEN
NOM-RELIS

FLOG LIFT

$\begin{array}{ll}0.0 & 0.9 \\ 0.0 & 1.0 \\ 0.0 & 1.0 \\ 0.0 & 1.0\end{array}$ 
ABANDONED GNSHORE OIL FIßLDS NITH OVER 250,000 BBI. CUMOLATIVE PRODUCTION

DST DTSC ABD. FLD NTH PIELD NAME

084 $1936 \quad 1952 \quad 263.72001$ DDGGAN
DEPTH GRAVIT

5,000

31.0

1 IIELD (S)
COMOI. PRCD. OF FIELD

545.172

cONTY

COC HR AN
COINTY : COCHRAN 
ABANDONED ONSHORE OIL FIELDS ITT OVER 250,000 BBL. COHOLATIVE PRODUCTICN

DST DISC ABD. FID NOM FIELD NAME

O8A 1956197463800500 MTNGERVIILE, B. (CANYON REEF)

DEPTH GRAVITY

9,077

36.5
ROM-RELLS

IEAR

1974

1973

1972

1971

1970

1969

1968

1967
1966

DST DISC ABD. FID NOH FIELD NAHE

OBA 1951197053702500 IINDSEY (STRARM)

\section{DEPTH GRAVITY}

10,948

45.2

\section{PLON LIPT}

$\begin{array}{ll}0.0 & 0.3 \\ 0.0 & 2.0\end{array}$

$\begin{array}{ll}0.0 & 2.0 \\ 0.0 & 2.0\end{array}$

$0.0 \quad 2.0$

0.03 .0

0.03 .0

$0.0 \quad 4.7$

$0.3 \quad 4.7$

0.05 .0
CTHO L. PROD. OP FI ELD

431,837

$\begin{array}{rrr}\text { OIL-PRD } & \text { E/RD } & \text { GAS-PRD } \\ & & \\ 310 & 2.5 & 92 \\ 3636 & 5.0 & 697 \\ 7146 & 9.8 & 1048 \\ 13432 & 12.3 & 1565 \\ 18314 & 16.7 & 2610 \\ 25254 & 23.1 & 5774 \\ 32199 & 18.9 & 10028 \\ 48390 & 26.5 & 13842 \\ 45084 & 24.7 & 16002\end{array}$

TEAR
1970
1969
1968
1967
1966

2 PIELD (S)

$\operatorname{coNTP}$

CA HSON
897.414

COONTY

CAHSON

$\begin{array}{lrrrr}\begin{array}{r}\text { NOH-REIIS } \\ \text { PION IIFT }\end{array} & \text { OIL-PRD } & \text { E/HD } & \text { GAS-PRD } \\ & & & & \\ 0.0 & 0.1 & 349 & 11.5 & 315 \\ 0.0 & 1.0 & 5189 & 14.2 & 4669 \\ 0.0 & 1.0 & 12089 & 33.1 & 11046 \\ 0.0 & 1.0 & 11276 & 30.9 & 10147 \\ 0.0 & 1.0 & 11998 & 32.9 & 11895\end{array}$

1,329,251 COMULATIVE PROD. PROM ABANDONBD PIELDS 
ABANDONED ONSHORE OIL FIEIDS WITR OVER 250,000 BBL. COUOLATIVE PROROCTION

DST DISC ABD. PLD NOM PIBLD MAME

$083 \quad 1940 \quad 1973 \quad 95417001$ DASSOR 66

\begin{tabular}{|c|c|c|}
\hline DBPTH & GEAVITY & co.go L. \\
\hline 6,900 & 32.0 & \\
\hline Y BAR & & $\begin{array}{l}\text { NOH-RELIS } \\
\text { PLOV IIPT }\end{array}$ \\
\hline $\begin{array}{l}1973 \\
1972 \\
1971 \\
1970 \\
1969 \\
1968 \\
1967 \\
1966\end{array}$ & & $\begin{array}{l}1.2204 .8 \\
1.2275 .5 \\
1.6285 .2 \\
1.3299 .8 \\
1.0307 .6 \\
1.3312 .6 \\
2.6292 .2 \\
4.6293 .7\end{array}$ \\
\hline
\end{tabular}

DEPTH GRAVITY

8,742

29.0
CDMUL. PROD. OP PIELD

$1,404,126$
ConNT

GAINES

$\begin{array}{lll}\text { OIL-PRD } & \text { E/RD } & \text { GAS-PBD } \\ & & \\ 1437015 & 19.1 & 2938395 \\ 2052893 & 20.3 & 4083830 \\ 1942039 & 18.5 & 3565209 \\ 2105984 & 19.2 & 3494388 \\ 2228862 & 19.8 & 3223484 \\ 2520992 & 22.0 & 2815343 \\ 2591760 & 24.1 & 3595001 \\ 2343017 & 21.5 & 2916303\end{array}$

COU NTY

GAINES

O8A 19561976 \$2233600 SEMIYOLE, R. (LEONARD)

YEAA
1976
1975
1974
1973
1972
1971
1970
1969
1968
1967
1966

\section{NOH-TELIS}

PION LIFT

$\begin{array}{ll}0.0 & 0.3 \\ 0.0 & 1.0 \\ 0.0 & 1.0 \\ 0.0 & 1.0 \\ 0.0 & 3.0 \\ 0.3 & 2.8 \\ 1.8 & 9.7 \\ 2.2 & 2.5 \\ 3.0 & 2.0 \\ 3.0 & 2.5 \\ 3.0 & 3.0\end{array}$

CIL-PRD E/RD

5536.1

$\begin{array}{rr}3665 & 10.0 \\ 4855 & 13.3\end{array}$

$\begin{array}{ll}4855 & 13.3 \\ 7159 & 19.6\end{array}$

$7159 \cdot 19.6$

$32614 \quad 29.8$

$53884 \quad 49.2$

$75705 \quad 57.9$

$104370 \quad 61.3$

$\begin{array}{rr}104370 & 61.3 \\ 722427 & 67.1\end{array}$

$141186 \quad 70.3$

$\begin{array}{ll}141186 & 70.3 \\ 119331 & 54.5\end{array}$
GAS-P AD

16
115
146
216
1904
2059
2645
2690
5629
5227
8715


ABANDONED ONSHORE OIL PIELDS RITH OVER 250,000 BBL. CO GOLATIVE PRODUCTION

DST DISC ABD. PLD NOM PTELD NAME

O8A $19531975 \quad 1964666$ AL SABROOK (RCLFCAMP)

DST DISC A SD. PLD NOG PIELD NAEE

08A $19561976 \quad 81913750$ SRAGRAVES (STRATM)
DEPTH GRAVITY CUUOL. PROD. OP PIELD CCONTY

$9,125 \quad 38.5 \quad 9.053,164 \quad$ GAINES

$\begin{array}{lrrrrr}\text { YEAR } & \begin{array}{r}\text { NOE-RELIS } \\ \text { PIOV LIPT }\end{array} & \text { OIL-PRD } & \text { E/R D } & \text { GAS-PRD } \\ & & & & & \\ 1976 & 0.0 & 0.9 & 4466 & 13.3 & 941 \\ 1975 & 0.0 & 0.8 & 6250 & 20.5 & 1374 \\ 1974 & 0.0 & 1.0 & 13516 & 37.0 & 2974 \\ 1973 & 0.0 & 1.0 & 14050 & 38.5 & 3094 \\ 1972 & 0.0 & 1.0 & 17745 & 48.6 & 3903 \\ 1971 & 0.0 & 1.0 & 16937 & 46.4 & 3727 \\ 1970 & 2.0 & 3.0 & 18390 & 10.1 & 4067 \\ 1969 & 2.0 & 3.0 & 25399 & 13.9 & 5678 \\ 1968 & 2.2 & 2.8 & 28912 & 15.8 & 6444 \\ 1967 & 3.0 & 2.0 & 26913 & 14.7 & 6070 \\ 1966 & 3.0 & 2.0 & 27521 & 15.1 & 6131\end{array}$

$\begin{array}{rcccc}\text { DEPTE } & \text { GRAVITI } & \text { COHOL. } & \text { PROD. OF FIEID } & \text { COOHTY } \\ 11,243 & 47.0 & & 913,031 & \text { GAIRES }\end{array}$

NOH- RELLS
YEAR PLON LIET

OIL-PRD EYRD GAS-PRD

$1976 \quad 0.0 \quad 0.1$

$1975 \quad 0.0 \cdot 1.4$

19740.02 .1

$1973 \quad 0.01 .8$

19720.02 .0

197100.03 .1

$197 \mathrm{C} \quad 0.04 .0$

19690.04 .0

$1968 \quad 0.04 .0$

$1967 \quad 0.01 .1$

1966

$\begin{array}{rrr}17 & 0.6 & 17 \\ 1340 & 2.6 & 200 \\ 2413 & 3.2 & 198 \\ 1214 & 2.0 & 22 \\ 2534 & 3.5 & 357 \\ 7009 & 6.2 & 472 \\ 12959 & 8.9 & 694 \\ 11460 & 7.8 & 687 \\ 20490 & 14.0 & 1068 \\ 28607 & 72.3 & 1137 \\ 26221 & 18.0 & 3466\end{array}$


ABANDONED ONSHORE OIL FIEIDS RITH OVER 250,000 BBL. COAOLAT IVE PRODUCTION

DST PTSC ABD. PLD NMM FIELD NAME

ORA $19521969^{\circ} 46134250$ JENR INS, NORTH (CANYON)
DEPTH

8,590

YE $\mathbf{A R}$

1969

1968

1967

1966

GR AV ITY

41.8

\section{NOM-RELLS
FLOR LIPT}

$\begin{array}{ll}0.0 & 0.7 \\ 0.0 & 1.0 \\ 0.0 & 1.0 \\ 0.0 & 1.0\end{array}$

OIL-PRD E/RD

$\begin{array}{ll}1104 & 4.5 \\ 2582 & 7.1 \\ 1756 & 4.8 \\ 2110 & 5.8\end{array}$

GA S-PRD

1154

3242

5218
DST DISC ABD. FLD NIT FIELD NAME

O8A 1961197695398500 RASSON, EAST (HOLFCAM, LORER)
DEPTH

8,765
GRAVITY

38.1
COMOL. PROD. OF PIELD

478,366
COO ATY

GAINES

YEAR
1976
1975
1974
1973
1972
1971
1970
1969
1968
1967
1966

\section{NOH-RELLS}

FLON LIET

$0.0 \quad 0.8$

0.01 .0

$0.0 \quad 1.0$

0.01 .0

0.01 .3

$0.0 \quad 3.4$

$0.0 \quad 4.0$

$0.0 \quad 4.0$

0.04 .0

0.04 .0

$0.0 \quad 4: 0$ $\begin{array}{lll}5244 & 17.2 & 11157\end{array}$

$\begin{array}{lll}4943 & 13.5 & 15631\end{array}$

$\begin{array}{lll}4886 & 13.4 & 16798\end{array}$

$\begin{array}{lll}5615 & 15.4 & 21404\end{array}$

$\begin{array}{lll}9275 & 19.1 & 19138\end{array}$

$\begin{array}{lll}15387 & 12.3 & 12177\end{array}$

$\begin{array}{lll}21878 & 15.0 & 9267\end{array}$

$\begin{array}{lll}17388 & 11.9 & 4751\end{array}$

$\begin{array}{lll}18114 & 12.4 & 11119\end{array}$

$3037220.8 \quad 18684$

$\begin{array}{lll}39301 & 26.9 & 29383\end{array}$ 
ABANDOHED ONSRORE OIL PIEIDS RITH OVER 250,000 BBL. COMULAT IVE PRODUCT ION

DST DISC ABD. FLD NOM FTELD NAME

OBA 1952196946134750 JPNRINS, NORTH (DEVONIAN)

DST. DISC ABD.

08. 19491970

77316426 ROBERTSON (DEVONIAN)

\begin{tabular}{|c|c|c|c|}
\hline DEPTH & GRAVITY & \multicolumn{2}{|c|}{ CU MOL. } \\
\hline 9,677 & 38.0 & & \\
\hline & & NOM-1 & IELLS \\
\hline YEAR & & & LI PT \\
\hline $\begin{array}{l}1969 \\
1968 \\
1967 \\
1965\end{array}$ & & $\begin{array}{l}0.0 \\
0.3 \\
0.2 \\
0.0\end{array}$ & $\begin{array}{l}0.0 \\
0.0 \\
0.1 \\
1.0\end{array}$ \\
\hline
\end{tabular}

DE PTH GRAVITY

10,050

36.0

YEA R

NTU-WELIS

PLOV IIFT

1970

1969

1969

1967

1966

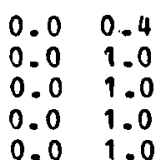

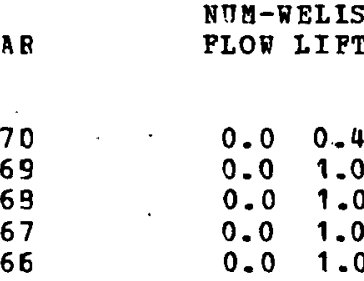

OD. CP FIRLD

469,155

$\operatorname{COONTY}$

GAI NES $\begin{array}{ll}0.0 & 0.1 \\ 0.0 & 1.0\end{array}$

COMOL. PROD. CIP PIELD

448.844
GA S-PRD

1
6
9
0

$\begin{array}{rr}1 & 0.0 \\ 1239 & 13.6 \\ 1334 & 14.6 \\ 3616 & 9.9\end{array}$

COONTY

EAINES

$\begin{array}{rr}\text { CIL-PRD } & \text { E/ } \\ & \\ 1505 & 9.9 \\ 7496 & 20.5 \\ 7234 & 19.8 \\ 9127 & 25 . \\ 10193 & 27.9\end{array}$

GAS-PRD

2637

10548

10548

10548

10548 
ABANDONED ONS YORE OIL. FIELDS NITH OVER 250,000 BEL. COUOLATIVE PRODOC?ION

DST DISC ABD. FID NTM PIELD HAHE

OBA $19561970 \quad 82225426$ SEMTNOLE (HOLFCA KP)
DEPTH

9, 120

YE AR
1970
1969
1968
1967
1966

DST DISC ABD. FLD NOM FIELD NAME

O8A 1958197582233800 SEMINOLE, W. (WOLFCAMP FB E)

\section{GR AV ITY}

27.4

444,289

CCUNTY

GAINES
DEPTH GRAVIT Y

9. 118
32.0
NOM-VEL
PIOT LI ZT

LOW LIET

0.01 .2

$0.7 \quad 0.3$

$1.0 \quad 0.0$

1.. 0.0 .0

$1.0 \quad 0.0$

CU YO 1. PROD. OF FIELD

431,507

$\begin{array}{rrr}\text { OII-PRD } & \text { E/HD } & \text { GAS-PRD } \\ & & \\ 12453 & 29.2 & 1268 \\ 50690 & 138.9 & 508 \\ 45621 & 125.0 & 258 \\ 41219 & 112.9 & 559 \\ 34106 & 93.4 & 1167\end{array}$

COONTY

GAI NES

YEAR

1975
1974
1973
1972
1971
1970
1969
1968
1967
1966

NOYI-VILI.S

PLOW छIET

$0.0 \quad 1.0$

$0.0 \quad 1.0$

$0.0 \quad 1.0$

$0.0 \quad 1.0$

$0.0 \quad 1.0$

0.011 .0

0.02 .0

0.02 .0

0.02 .5

0.03 .0
OIL-PRD E/WD GAS-PRD

$\begin{array}{rrr}6572 & 18.0 & 198 \\ 6607 & 18.1 & 199 \\ 8319 & 22.8 & 251 \\ 9724 & 26.6 & 310 \\ 10562 & 28.9 & 335 \\ 11399 & 31.2 & 349 \\ 17010 & 23.3 & 2058 \\ 19139 & 26.2 & 1982 \\ 26735 & 29.3 & 1914 \\ 38113 & 34.8 & 2914\end{array}$


ABANDONED ONSHORE OIL FIBLDS ITH OVER 250,000 BBL. CUHOLATIVE PRODUCTICA

DST. DISC ABD. FLD NOM FIELD HAME

OQ 19561972 S1920666 SEAGRAVES, R. (STRANN)

$$
\begin{array}{rr}
\text { DEPTE } & \text { GRAVITI } \\
11,454 & 44.0
\end{array}
$$

covor

L. FROD. OP FIELD

\begin{tabular}{|c|c|c|}
\hline EAB & $\begin{array}{l}\text { YOS-D } \\
\text { PLOR }\end{array}$ & $\begin{array}{l}\text { EL IS } \\
\text { LI PT }\end{array}$ \\
\hline $\begin{array}{l}1972 \\
1971 \\
1970 \\
1969 \\
1968 \\
1967 \\
1966\end{array}$ & $\begin{array}{l}0.0 \\
0.0 \\
0.0 \\
0.0 \\
0.0 \\
0.0 \\
0.0\end{array}$ & $\begin{array}{l}1.9 \\
2.0 \\
3.0 \\
2.0 \\
2.0 \\
1.0 \\
1.0\end{array}$ \\
\hline
\end{tabular}

424.238

CODKTY

GAIMES

DEPTE GRAVITI

8,861

46.2
COMUL. PROD. OP FIBLD

410,076
GAS-P BD

$\begin{array}{rrr}2696 & 3.9 & \\ 7866 & 10.8 & 8 \\ 12904 & 11.8 & 14 \\ 17574 & 24.1 & 33 \\ 20659 & 28.3 & 24 \\ 5361 & 14.7 & 24 \\ 5233 & 14.3 & 12 \\ & & 0\end{array}$

coomet

caings

O8: 1957197277318333 ROBERTSOH, MORTH (CAMYOB)

MOA-DELIS

FLOD IIPT

$0.0 \quad 0.1$

$0.0 \quad 0.2$

0.01 .0

0.01 .0

$0.0 \quad 1.0$
CII-PRD E/UD

GAS-PBD

$\begin{array}{rr}106 & 3.5 \\ 540 & 8.9 \\ 6781 & 18.6 \\ 6269 & 17.2 \\ 6579 & 18.0 \\ 5553 & 15.2\end{array}$


ABANDONED ONSHORE OIL FIZIDS IITH OVER 250,000 B9L. COEULATIVE PRODUCTICY

DST DISC ABD. PLD NTI PIRLD NAME

ORA 1970

65971500 MDRHAN, S. (HOLFSAMP)

DST DIS A AD. PLD NOY PIELD MAGE

O88 $19641969 \quad 96408332$ RESCOTT (HISS.)

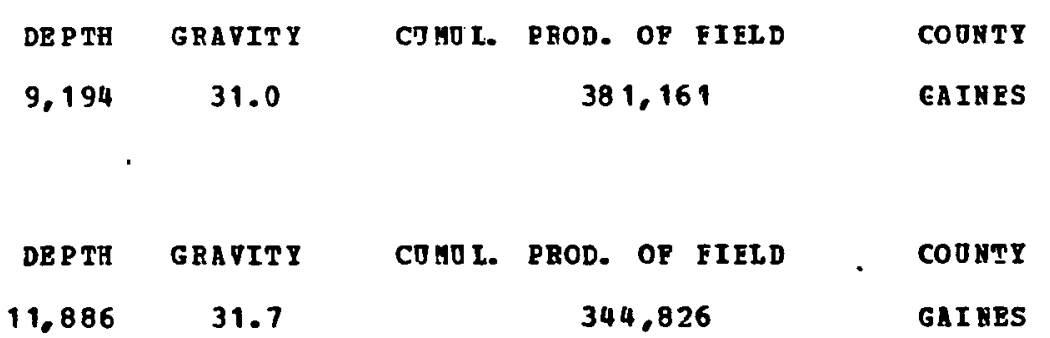

Y E AR

MOA-RELS

PLOD LIPY

$\begin{array}{ll}0.6 & 0.0 \\ 1.0 & 0.0 \\ 0.3 & 0.0 \\ 1.0 & 0.0\end{array}$

OIL-PRD E/RD

25159118.2

64440176.5

57831633.8

55532152.1
GAS-P RD

1441

3076

2715
DST DISC AD. FLD NTM PIELD NAME

OBA 1961197316862333 CHAMPHON, NORTH (STRAHW)

$\begin{array}{rcccc}\text { DEPTH } & \text { GRAYITY } & \text { COAUI. } & \text { PROD. OP FIELD } & \text { CODHTY } \\ 11.069 & 42.4 & & 341.773 & \text { GAIRES }\end{array}$

RUM-DELIS

PLOD LIFT

$\begin{array}{ll}0.0 & 0.6 \\ 0.0 & 1.0 \\ 0.0 & 3.0 \\ 0.0 & 3.0 \\ 0.0 & 3.0 \\ 0.0 & 3.0 \\ 0.0 & 3.0 \\ 0.0 & 3.0\end{array}$

OIL-PRD E/RD

$3249 \quad 15.3$

$11888 \quad 32.6$

1289711.8

$15550 \quad 14.2$

2346321.4

$27968 \quad 25.5$

3298230.1

$40148 \quad 36.7$
GA S-P RD

325

1920

2803

9660

11123

16513

10830

15371 
ABANDONED ONSHORE OIL FIELDS RTTH OVER 250,000 BBL. CUMOLATIVE ERODOCT ION

DST DISC ABD. FLD NTM PIELD NAME

OSA 1965197179009333 ROSSELL, SW. (DEVONIAN)
DEPTH

11,220

GRAV ITY

45.6

IEAR

1971

1970

1969

1968

1967

1966
OD. OF FIBLD

3190,192

CCUNTY

GAINIS
DST DISC ABD. FLD NOM FIELD NAME

08: 1959195977316568 ROBE RT SON (ELLEN BURGER)

DST DISC ABD. FLD NOM PIELD NAME

O8. 195819712406333 AMRON, NORTH (ST RAWM)
NOM- RELLS
FIOU LIFT

$0.0 \quad 2.0$

$0.0 \quad 2.0$

$0.0 \quad 2.7$

$0.0 \quad 3.5$

$0.2 \quad 3.7$

\section{DE PTE \\ 11,900 \\ GRAVITY \\ 40.0 \\ DE P TA \\ GRAVITY}

11,310

46.0

COMUI. PRCD. OF FIELD

273,153

OIL-PFD E/W E

GA S-P RD

$\begin{array}{rr}8357 & 11.4 \\ 13374 & 18.3 \\ 18748 & 19.3 \\ 29250 & 22.9 \\ 47753 & 32.7 \\ 1122472 & 85.7\end{array}$

610

732
319

24

2604

16886

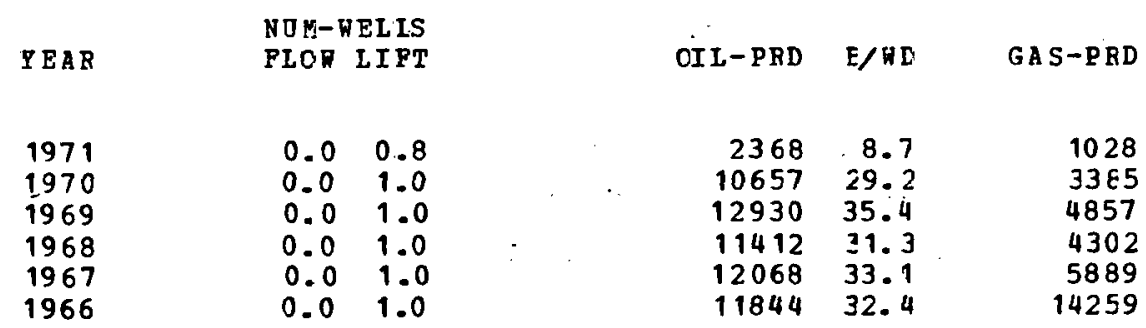


ABANDOKED ONSHORE OIT FIELDS ITH OVER 250,000 BBL. COHULATIVE PRODUCTICE

DST DISC ABD. PID MIM FIELD NAKE

ORA 1955197435200500 GLASCO, N. (DEVONIAN)

$\begin{array}{rcccc}\text { DEPTH } & \text { GRAVITY } & \text { COEDI. FROD. OF FIELD } & \text { COUHTY } \\ 12,608 & 37.0 & 254,006 & \text { GAINES }\end{array}$

YEAB

KOH-TELS
PLOD LET

OIL-FRD E/WD

GAS-PBD

1974

1973

1972

1971

1970

1969

1968

1967

1966

19 PIELD (S)

$\begin{array}{rrr}6858 & 18.8 & 12 \\ 2663 & 8.0 & 19 \\ 240 & 0.7 & 23 \\ 3780 & 10.4 & 34 \\ 1770 & 4.8 & 588 \\ 5708 & 15.6 & 12 \\ 5657 & 15.5 & 12 \\ 7206 & 19.7 & 12 \\ 6702 & 18.8 & 12\end{array}$

$\because \because$ COTHTY: GAIBES

46,826,767 COHOLATIVE PROD. PROK ABABLOBBC FIEIDS 
A5ANDONED ONSHORE OII PIELDS VTTH OVER 250,000 BBL. COMDLATIVE PRODOCTICN

DST DIST ABD. PLD NUM PTELD NALE

OBA $1954 \quad 1975 \quad 47758333$ JTSTICEBURG (GLORIETA)

DST DISC ABD. FID NDM FIELD NAME

$0817756 \quad 1974 \quad 25544500$ DORTARD (SAN ANDRES)

\begin{tabular}{|c|c|c|c|c|}
\hline DEPTH & GRAVITY & COMOL. & PROD. OF FIELD & CCUNTY \\
\hline 2,48 & $40: 0$ & & $3,2 \in 8,423$ & GARZA \\
\hline
\end{tabular}

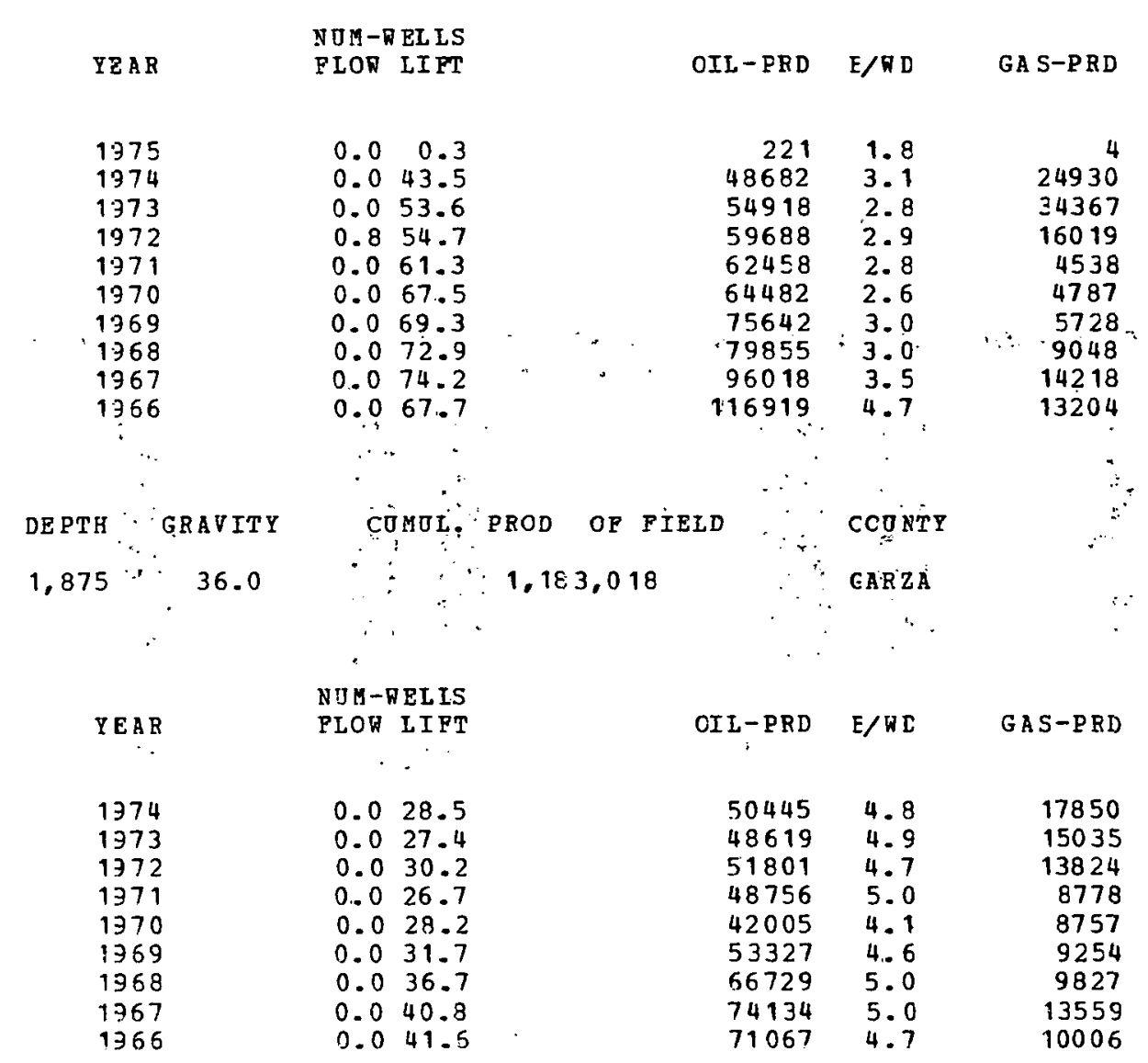


ABANDONED ONSHORE OIZ FIELDS MITH OVER 250,000 BBI. CUMOLAT IVE PRODOCTION

DST DIST ARD. FLD NDM FIELD NAME

OS $1958 \quad 1975 \quad 88611142$ TEAS (ELLEN BURGER)

DST DISC BD. FLD NOM" "IELD NAME

OQ4 1958196738866333 HAPPY (ELLENRDRGER)

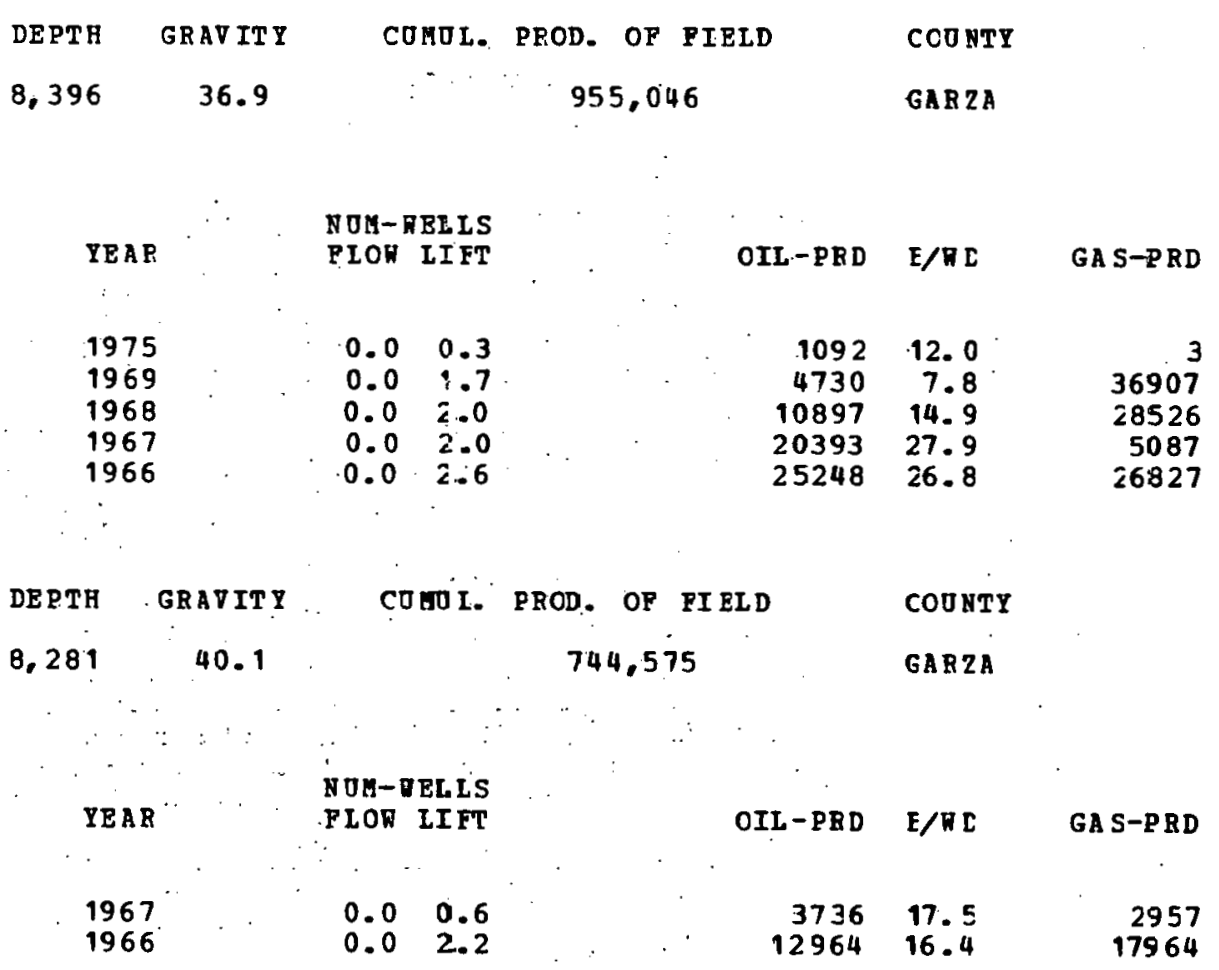


ABAYDONBD OHSHORE OIL PIELDS DITH OVER 250,000 BBL. COAOLATIVE PRODUCTIOH

DST DISC ABD. PLD NOA PIELD HARE

OB 19581972.98611284 TEAS (ELLBABORGRB, RORTB)

$\begin{array}{cc}\text { DEPTH } & \text { GRAVITY } \\ 8,538 & 39.6\end{array}$

COHOL. PROD. OE FIELD

336,210

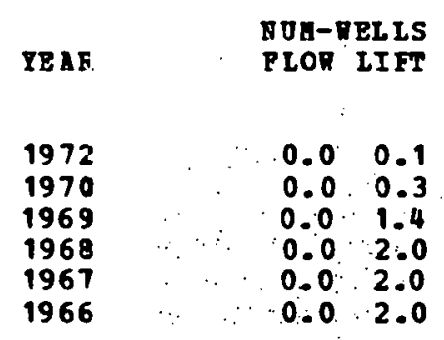

5 FIEID (S)
$\operatorname{CCONTY}$

GAR 2 A
$6,487,272$ COMOLATIVE PROD. PROM ABAHLOHEC PIELDS 
ABANDONED ONSHORE OIL PIEIDS VITH OVEF 250,000 BBL, COUOLATIVE PRODICTICA

DST DISC ABD. FLD NOI FIELD NAUE

08A 1948
3554500 ARICK (DOLFCAAP)

coonti:
DEPTH

4,798

PIELD (S)
GRAVITY

42.2

CIMOL. PROD OP. FIELD

385,964

CD NTY

EA L. $\mathrm{E}$ 
ABANDONED ONSHORE OII PIELDS RITH OVER 250,000 BBL. COGOLATIFE PRODJCTIOA

DST DISC ABD. PLD NTE PIELD NAHE

$084^{\circ} 1950 \quad 1972 \quad 19346852$ COSDELL $(4900)$

\begin{tabular}{|c|c|c|c|}
\hline DEPTA & GRAVITY & \multicolumn{2}{|c|}{ CO 1 . } \\
\hline 4.910 & 43.2 & & \\
\hline$\because "$ & & $808-8$ & ELIS \\
\hline IEAB & 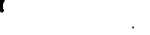 & FLON & LIF \\
\hline $\begin{array}{l}1972 \\
1971 \\
1970 \\
1969 \\
1968 \\
1967 \\
1966\end{array}$ & & $\begin{array}{l}0.0 \\
0.0 \\
0.0 \\
0.0 \\
0.0 \\
0.0 \\
0.0\end{array}$ & $\begin{array}{l}1.02 \\
3.0 \\
3.6 \\
4.0 \\
4.0 \\
4.0 \\
4.5\end{array}$ \\
\hline
\end{tabular}

DEPTH

GRAVITY

4,538
38.6
COUOL. PBOD. OF FIELD

263,479

COOBTY

KEET

$972,858 \quad$
OrI-PRD

951
3881
9423
16751
22660
28925
37154

DST DISC ABD. FLD NOH PIELD NABE

O8A 1952197619347750 COSDELI, EAST (COGDELI SAND)
GAS-PRD

36
30
12
407
2059
2565
5461

\begin{tabular}{lll} 
YEAB & \multicolumn{2}{c}{ ROA-RELIS } \\
PLOR & LI FT \\
& & \\
1976 & 0.0 & 3.0 \\
1975 & 0.0 & 3.0 \\
1974 & 0.3 & 2.3 \\
1973 & 0.0 & 2.0 \\
1972 & 0.0 & 3.0 \\
1971 & 0.0 & 3.0 \\
1970 & 0.0 & 3.0 \\
1969 & 0.0 & 3.0 \\
1968 & 0.0 & 3.0 \\
1967 & 0.0 & 3.0 \\
1966 & 0.0 & 3.0
\end{tabular}

$\begin{array}{rrr}\text { OIL-PRD } & \text { E/RD } & \text { GAS-PRD } \\ & & \\ 871 & 0.8 & 12 \\ 1253 & 1.1 & 12 \\ 1488 & 1.6 & 10 \\ 986 & 1.4 & 12 \\ 2716 & 2.5 & 12 \\ 3022 & 2.8 & 17 \\ 3028 & 2.8 & 422 \\ 3840 & 3.5 & 880 \\ 3899 & 3.6 & 988 \\ 5131 & 4.7 & 1053 \\ 6375 & 5.8 & 1078\end{array}$


ABAMDOHED ONSBORE OIL FIEIDS RITH OVER 250,000 BEL. COHOLATIVE FROCOCT ION

DST DISC ABD. PLD NOH PIELD HAEE

O8\ 195519713335500 ARAR [ELLEMBORGEG]

DST DISC ABD. PID NOE PIBLD RABE

081 1957. 197490846500 TONLB. (CANYON)

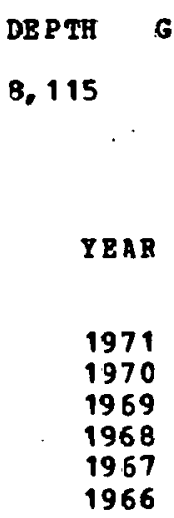

DBPTH

8, 115

\section{1}

1970

1969

1968

1966

GRAVITY

41.0

COHEI. PROD. OF FIELD

coontr
NOH-DELIS
PLOR IIFT

$\begin{array}{ll}0.0 & 1.2 \\ 0.0 & 2.0 \\ 0.0 & 2.0 \\ 0.0 & 2.5 \\ 0.0 & 3.0 \\ 0.0 & 3.0\end{array}$

DE PTR

GRAVIT Y

7. 116

40.0
SCORRY

687.094

$\begin{array}{rr}\text { OIL-FRD } & \text { E/RD } \\ & \\ 3206 & 7.5 \\ 9854 & 13.5 \\ 12930 & 17.7 \\ 14394 & 15.8 \\ 18537 & 16.9 \\ 26845 & 24.5\end{array}$

CUMOL PROD. OP FIELD

430,240

COOHTY

SCOBRI
GAS-PBD

1796

3885

6416

6416 0

\begin{tabular}{|c|c|c|}
\hline YBAR & $\begin{array}{l}\text { NOH- } \\
\text { PIOA }\end{array}$ & $\begin{array}{l}\text { EL IS } \\
\text { II FT }\end{array}$ \\
\hline $\begin{array}{l}1974 \\
1973 \\
1972 \\
1971 \\
1970 \\
1969 \\
1968 \\
1967\end{array}$ & $\begin{array}{l}0.0 \\
0.0 \\
0.0 \\
0.0 \\
0.0 \\
0.0 \\
0.0 \\
0.0\end{array}$ & $\begin{array}{l}1.5 \\
3.13 \\
3.4 \\
4.13 \\
4.0 \\
4.0 \\
4.0 \\
4.0\end{array}$ \\
\hline
\end{tabular}

OIL-PRD E/RD

GAS-P BD

$\begin{array}{ll}3472 & 6.3 \\ 7163 & 6.5\end{array}$

$\begin{array}{ll}7163 & 6.5 \\ 6260 & 5.0\end{array}$

$\begin{array}{rr}6260 & 5.0 \\ 15045 & 10.3\end{array}$

$16915 \quad i 1.6$

$18434 \quad 12.6$

$18298 \quad 12.5$

1900413.0

1977813.5 
ABANDONED ONSBORE OIL FIELDS ITA OVER 250,000 BBL. COHULATIVE PRODOCTICH

DST DISC ABD: PLD NTM PIBID NAME

OB. $19571960 \cdot 40716666$ HERALEIGH (6200)

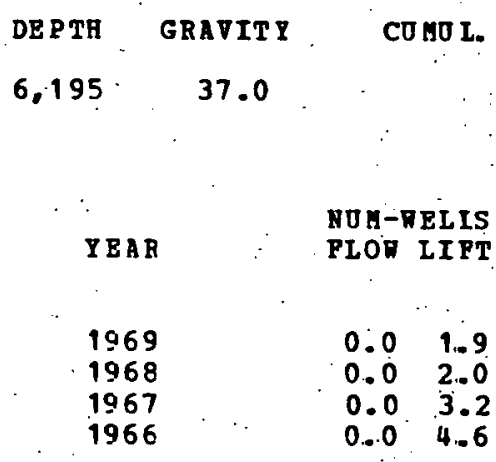

COOHTY

SCORRY
$30-1,214$

$\begin{array}{rr}\text { OIL-PRD } & E / R D \\ & \\ 1401 & 2.0 \\ 1920 & 2.6 \\ 3996 & 3.5 \\ 6579 & 3.9\end{array}$

GAS-P BD

1257

1944

2889 4496 
ABANDONED OESHORE OIL FIEIDS ITH OVER 250,000 BBL: COMULATIVE PRODOCTION

DST DISC ABD. FID NOM FIELD NAHE

O8A $1954 \quad 1971$

$\begin{array}{cc}\text { DE PTH } & \text { GRAVITY } \\ 8,846 & 42.5\end{array}$

DE PTH

8,846

YEAR

1971

1970

1969

1968

1967

1966

DST DISC ABD. FID NOM PIELD NABE

DEPTE

GRAVITY

96184500 ตELLAAN, NW. (SI LERC-DEV ONIAM)
12,763

2 IIELD (S)

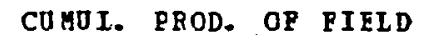

0.0 .2 .7

$0.04 . .0$

0.04 .0

$0.0-4.0$

0.06 .0

CUMU I. PROD. OP FIELD

303.680

$\begin{array}{rr}7434 & 7 . \\ 19955 & 13 . \\ 22495 & 15 . \\ 30080 & 20 . \\ 45153 & 33 . \\ 52700 & 24 .\end{array}$

COUNTY

TER RY

34.2 
AEANDONED ORSHORE OIL PIELDS ITH OVER 250,000 BBL. COHULATIVE PRODUCTICE

DST DISC ABD: PLD ITI FIELD NAEE

OAA $1954 \quad 1967 \quad 71823333$ PLATANG (DEVOHIAM)

DST DTSC ABD. FLD NGS PIELD NAHE

OB\ 1954197212160800 BRONCO (DOLPCAMP)

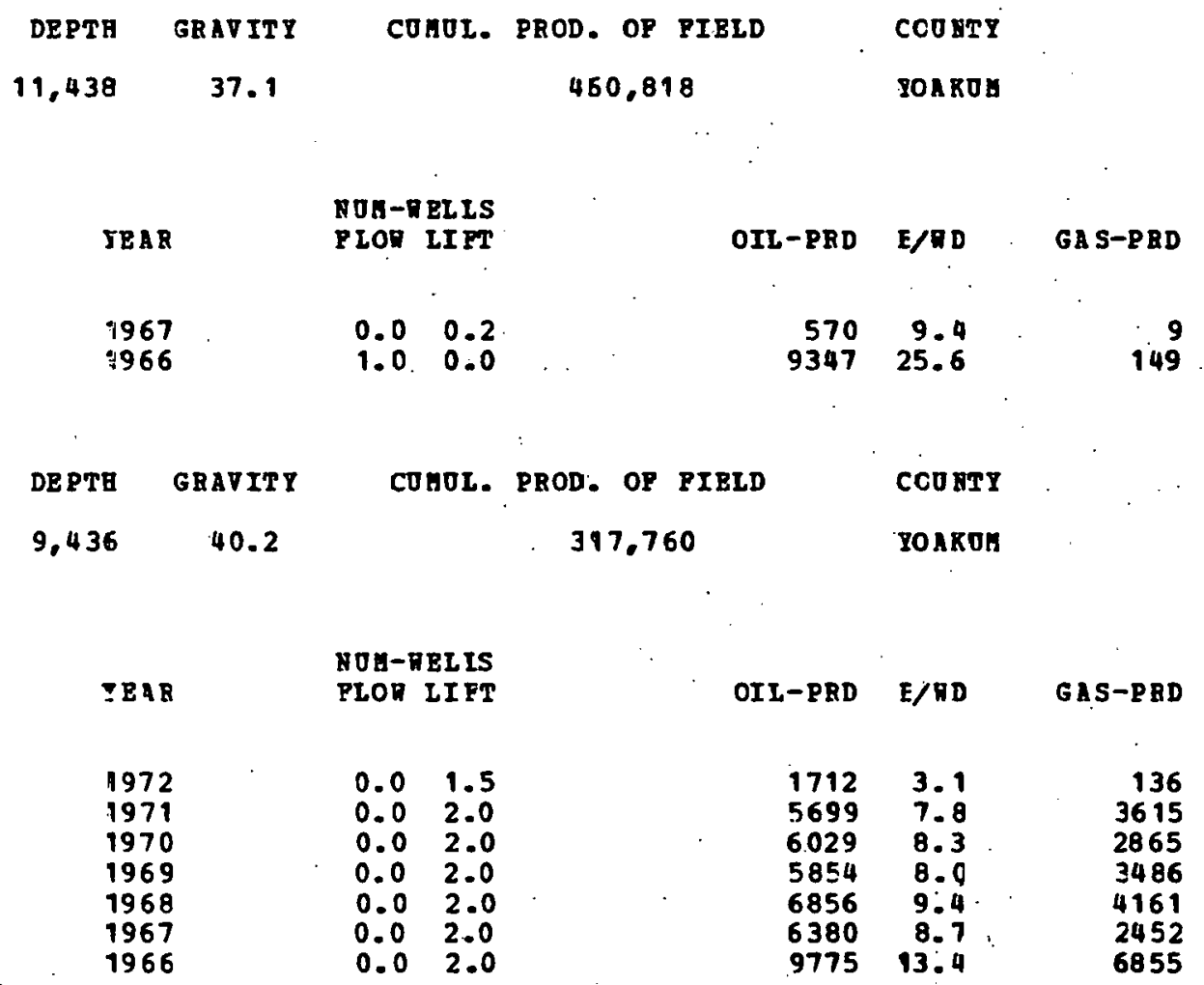


ABANDONED CNSHORE OIL FIELDS RITH OVER 250,000 BBL. COHOLATIVE PRODUCTION

DST DISC ABD. FID NTH FIDID NAME

ORA 1960197144379666 INDIAN CAMP (DEVONIAN)

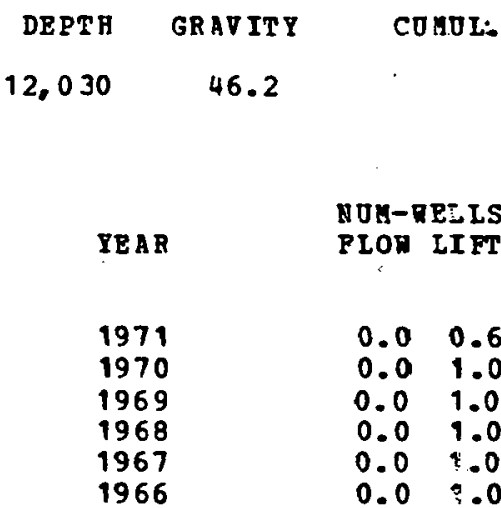

3 PIELD (S)

\begin{abstract}
COJUTY: YOAKD
\end{abstract}
DISTRICT OBA

41 PIEIDS
$\operatorname{ccoNTY}$
YOAROX
286,701

OIL PRD E/RC

GA S-PRD

$\begin{array}{rrr}2514 & 11.8 & 906 \\ 5606 & 15.4 & 1956\end{array}$

6147

$1102130.2 \quad 2142$

$2014255.2 \quad 2064$

$14372 \quad 39.4 \quad 2064$

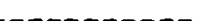

$1,065,279$ CUMULATIVE PBOD. FROM ABAYLONBD RIEIDS

61,785,463 COMOLATIVE PROD. FROA ABAYDONBD FIELDS 
ABAKDONED ONSHORE OIL FIELDS NITH OVER 250,000 BBL. COUOLATIVE PRODOCT. ION

DST DISC ABD. PLD NOL PIBLD NAME

091949197424208666 DEHOSS (STRANM)

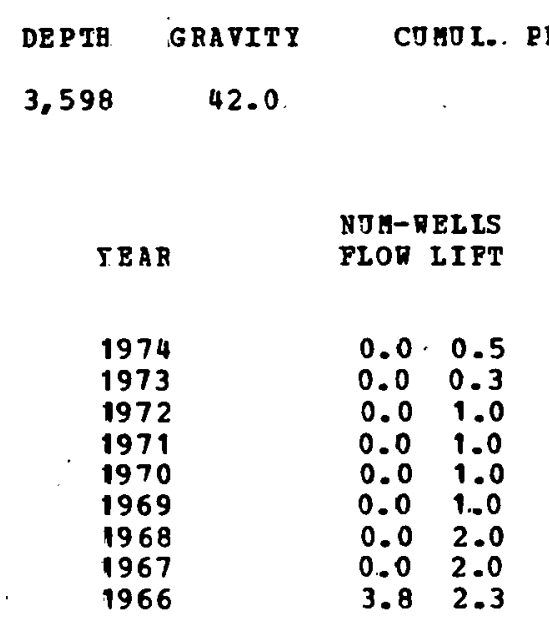

DEPTE GRAVITT

3.900

41.0
COHOL: PQOD..OF FIELD

667,686

$\begin{array}{rr}\text { OIL-PRD } & E / R D \\ & \\ 408 & 2.2 \\ 307 & 2.5 \\ 1102 & 3.0 \\ 1686 & 4.6 \\ 2008 & 5.5 \\ 1566 & 4.3 \\ 2940 & 4.0 \\ 6571 & 9.9 \\ 5491 & 2.5\end{array}$

COOMTY

ARC BER

091948197575962852 RENO (STRAaN 3900 SAND)

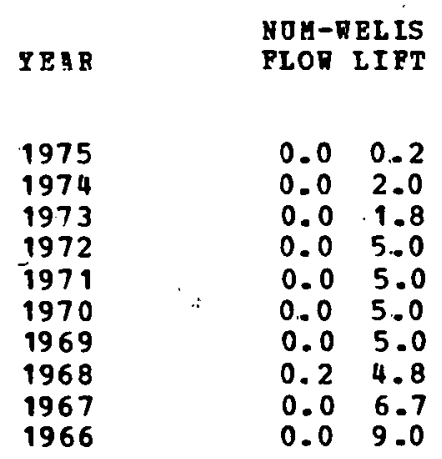

$\begin{array}{rr}\text { OIL-PRD } & \text { E/WD } \\ & \\ 111 & 1.8 \\ 658 & 0.9 \\ 751 & 1.1 \\ 2161 & 1.2 \\ 3201 & 1.8 \\ 5291 & 2.9 \\ 8504 & 4.7 \\ 26972 & 14.8 \\ 6503 & 2.7 \\ 7937 & 2.4\end{array}$

GAS-P RD

8
31
109
189
202
242
349
595
0

GAS-PRD

1
12
14
123
193
276
302
487
696
0


ABANDONED CNSHORE OIL FI

DST DISC ABD. FLD NOM PIBLD NAME

$09 \quad 1949$

DST DISC ABD. FLD NOA PIELD NAHE

$09 \quad 1951 \quad 1968 \quad 60156500$ MPDDERS (STRAMM)
94049750 VOGTSBERGER (STRAWN)

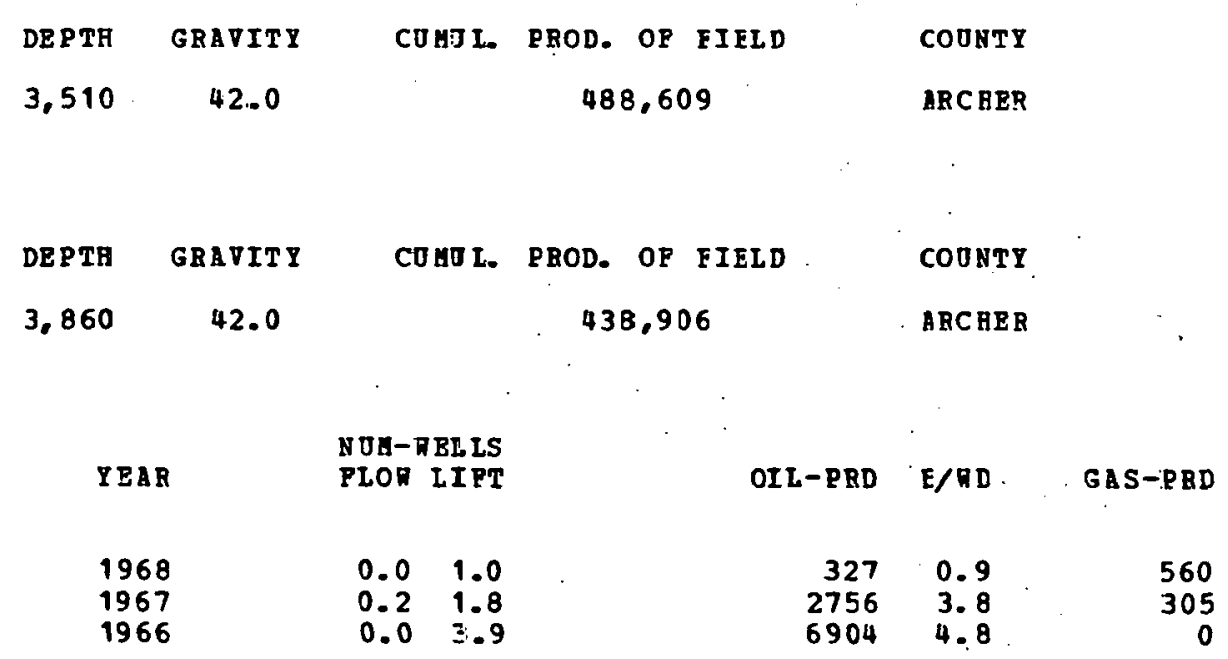

DEPTH GRAVITY COMUL. PROD. OF PIELD CCUNTY

2,533

42.6

372,894

ARCBER

091950

46879600 JOHN KAY (CANYON, OPPER)

DE P TH

GRAVITY

42.0
CUMTL. PROD, OF FIELD

342,750
COONTY

$09 \quad 1954196890950498$ TRANS-CONTINEHTAI (MISS. 5550) 5,550

$\begin{array}{rrr}2029 & 2.4 & 3777 \\ 8476 & 7.7 & 13200 \\ 13428 & 12.3 & 13200\end{array}$


ABANDONED OHSHORE OIL FIELDS ITTR OVER 250,000 BBL. COUOLATIVB PRODOCTIOA

DST -DISC ABD. PLD RTM FIELD NABE

09. 1950197160269400 MEGARGEL (MISSISSIPPI IIHE)

DEPTE GRATITY

5.072

43. 0

YEAR

1971

1970

1969

1967

1966
CUHOL. PROD. OP PIBLD

328,557

FUH-RELIS PLOQ LIFT

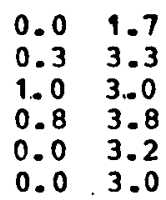

$0.3 \quad 3.3$

$1.0 \quad 3.0$

$0.0 \quad 3.2$

$0.0 \quad 3.0$

DEPTH GRAVITY

3,550

42.0

COMUL. PROD. OF PIELD

269.838

$\begin{array}{rr}\text { OIL-PRD } & \text { E/RD } \\ & \\ 1877 & 2.9 \\ 5608 & 4.3 \\ 9968 & 6.8 \\ 13781 & 8.2 \\ 11286 & 9.8 \\ 9889 & 9.0\end{array}$

GA S-PRD

12
28
36
39
36
0

$0919481974 \quad 46879400$ JORN KAY (CANYOH, LONER) $\operatorname{cod} \operatorname{RT}$

ABC AB B $\operatorname{cov} \operatorname{RTY}$

BRC BER

Y

1974
1973
1972
1971
1970
1969
1968
1967
1966

NOM-RELIS

PLOR LIFT

$0.0 \quad 2.3$

$\begin{array}{ll}0.0 & 2.0 \\ 0.0 & 7.0\end{array}$

0.06 .8

0.06 .0

0.06 .0

0.06 .0

0.06 .0

0.06 .0 
ABANDONED OBSHORE OII PIELDS IITH OVER 250,000 B3L. CUUOLATIVE ERODUCTION

DST DISC ABD. FLD NOM FIBLD NABE

$0919481963 \quad 64665001$ YEAL

DST DISC ABD. FLD NOM PIELD NAHE

$09 \quad 1948$

13792001 BURNS-ICRERT

DST DISC ABD. PLD NOB FIELD BAHE

$091951 \quad 196720163500$ CONNER (UISSISSIPDI IIHE)

\section{DE PTA}

5,208

GRAVITY

47.0

DEPTH

4.509

GRAVITY

41.5

CEUOL. PROD. OF FIELD

253,698

DE P TH

5,150

GRAVIT I

43.. 0

CUMOL. PROD. OF FIELD

253,394

COONT Y

\section{ARC BER \\ CON NTY \\ AECHER \\ CCONTY \\ ARC BER}

YEA

1967

1966

COONTT: ARCHER

11 PIELD (S)

$$
\text { MOH-RELLS }
$$

$$
\text { CII-ERD E/RD }
$$

GAS-PBD

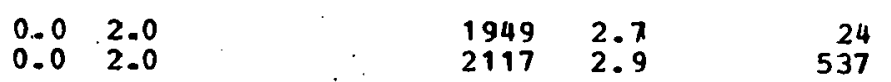


ABANDONED ONSHORE OIL FIEIDS AITH OVRR 250,000 BBI. COMULATIVE PRODOCTICN

DST DISC ARD. PLD NOM PIELD NAGE

091952197182479500 SEYHOOR, EAST (STRANN)

DST DISC ABD. PLD NOE FIELD NAME

$09 \quad 1951 \quad 1964 \quad 82478565$ SEYMOOR (STRARH)

DST DISC ABD. FLD NOM FIELD NAME

$09 \quad 1953 \quad 75887500$ RE NDHA M (MISSISSIPPI)

$\begin{array}{ccccc}\text { DEPTH } & \text { GRAVITY } & \text { COMOL. } & \text { PROD. OF PIELD } & \text { CCOHTY } \\ 4,717 & 40.0 & & 2,065,082 & \text { EATLOR }\end{array}$

YEAR

NOK-RBLIS

FIOR LIFT

OIL-PRD E/RE

GA S-PRD

1971
.1970

1970
1969

1969
1968

1967

1966

$\begin{array}{rr}0.0 & 1.0 \\ 0.0 & 2.0 \\ 0.0 & 3.0 \\ 0.0 & 5.5 \\ 0.0 & 7.0 \\ 0.0 & 12.4\end{array}$

8782.4

$2627 \quad 3.6$

76336.0

9337
17927

$179227 . \mathrm{C}$

300

0.07 .0

38327

8.5

DE PTH

GRAVITY

COMUL. PROD. OF PIELD

$\operatorname{cod} \operatorname{sTr}$

4,747

40.0

850.714

EAY LOR

DEPTH GRAVITI

COHOI. PROD. OF EIELD

COOHTY

5,402

39.8

458,253

EAYLOR 
ABANDONED ONSHORE OIL PIEIDS MITH OVER 250,000 BEL. CUMOLAT IVE FROLOCTICN'

DST DISC ABD. PLD NOA PIELD NAGE

$09 \quad 1948 \quad 1974 \quad 481001$ ACRE

DST DISC ABD. FLD NUE PIELD NAYE

$09 \quad 1951 \quad 197365357600$ NPRPORT (STRARN)

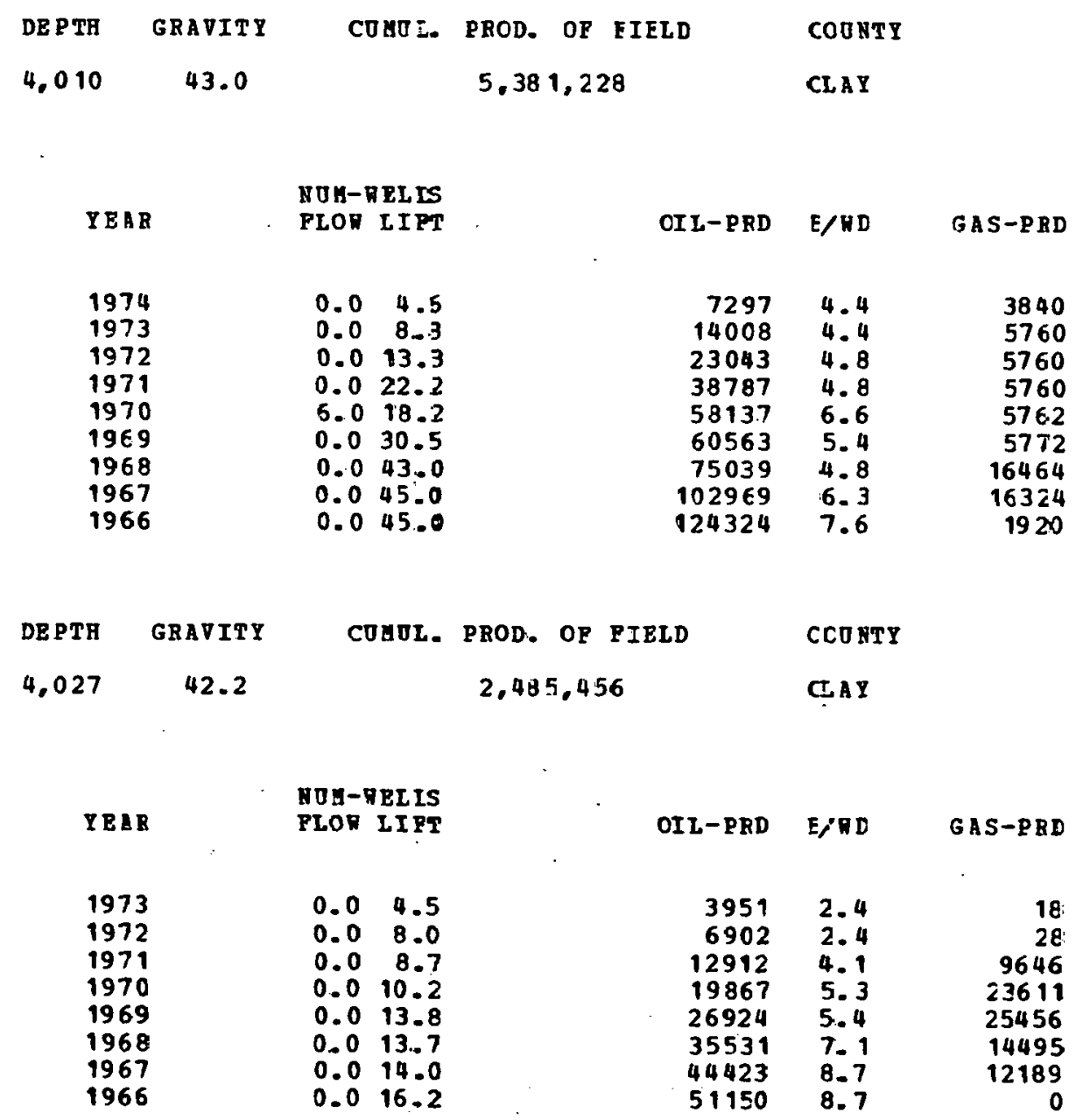


ABANDORED ONSHOBE OIL PIELDS ITH OVER 250,000 BBL. CUMULATIVE PRODUCTICH

DST DISC ABD. PLD NOM PIELD MAHE

$09 \quad 1947 \quad 1974 \quad 12749750$ PRTART (SIMPSON)

$\begin{array}{ll}\text { DEPTH } & \text { G } \\ 6,261 \\ \\ \\ \text { IEAR } \\ \\ 1974 \\ 1973 \\ 1972 \\ 1971 \\ 1970 \\ 1969 \\ 1968 \\ 1967 \\ 1966\end{array}$

DST DISC ABD. PID NTM PIELD NAGE

$09 \quad 19551975 \quad 50897666$ LA-PAN (IISSISSIPPIAN)

$6,24 ?$

\begin{abstract}
GRAVITY
\end{abstract}
43.8

CU RU L. PBOD. OP FIELD

939,798

COONTY

CIAY

\begin{tabular}{|c|c|c|c|c|}
\hline YEAR & $\begin{array}{l}\text { RTA-RELIS } \\
\text { PLOD LIFT }\end{array}$ & .CII-PRD & $E / D D$ & GAS-PBD \\
\hline $\begin{array}{l}1974 \\
1973 \\
1972 \\
1971 \\
1970 \\
1969 \\
1968 \\
1967 \\
1966\end{array}$ & $\begin{array}{ll}0.0 & 1.7 \\
0.0 & 2.9 \\
0.0 & 3.0 \\
0.0 & 3.0 \\
0.0 & 3.8 \\
0.0 & 4.0 \\
0.0 & 4.6 \\
0.0 & 5.0 \\
0.0 & 5.9\end{array}$ & $\begin{array}{r}1476 \\
2792 \\
3975 \\
5443 \\
5752 \\
6288 \\
8625 \\
9097 \\
11631\end{array}$ & $\begin{array}{l}2.4 \\
2.6 \\
3.6 \\
5.0 \\
4.9 \\
4.3 \\
5.2 \\
5.9 \\
5.4\end{array}$ & $\begin{array}{r}600 \\
885 \\
900 \\
900 \\
2835 \\
3362 \\
3709 \\
8635 \\
0\end{array}$ \\
\hline
\end{tabular}

$\begin{array}{cccc}\text { DEPTE } & \text { GRAVITY } & \text { CONOL. PROD. OF PIEID } & \text { CODHTY } \\ 6,247 & 45.0 & 902.792 & \text { CLAY }\end{array}$

45.0

902,792

CLAI

\begin{tabular}{lrl} 
& \multicolumn{2}{c}{ NOH-RELIS } \\
TEAR & PLOR IIPT \\
& & \\
1975 & 0.0 & 1.06 \\
1974 & 0.2 & 2.7 \\
1973 & 0.0 & 3.0 \\
1972 & 0.0 & 3.0 \\
1971 & 0.0 & 3.02 \\
1970 & 0.5 & 4.5 \\
1969 & 1.0 & 5.0 \\
1968 & 1.8 & 4.2 \\
1967 & 2.0 & 4.0 \\
1966 & 3.0 & 3.0
\end{tabular}

$\begin{array}{rrr}\text { OII-PRD } & \text { E/RD } & \text { GAS-PBD } \\ & & \\ 251 & 0.4 & 12604 \\ 1047 & 1.0 & 24844 \\ 1424 & 1.3 & 30359 \\ 1247 & 1.1 & 24203 \\ 1593 & 1.4 & 30357 \\ 2456 & 1.3 & 31501 \\ 2621 & 1.2 & 54105 \\ 3469 & 1.6 & 1 C 9458 \\ 7726 & 3.5 & 85162 \\ 16727 & 7.6 & 0\end{array}$


ABA NDONED ONSHOEE OIL FEELDS RITH OVER 250,000 BBב. COUOLATIVE PRODOCTICA

DST DISC ABD. PLD NTM FIELD NAME

091948

12749500 BRYANT (EDHARDS)

DST DISC ABD. FID NDM PIELD NAHE

091046196940014666 HEARD (ELLENBORGER)

DST DISC ABD. FID NOM. FIBID NAME

$091943 \quad 99056500$ VYNN (MISS. LIME)

DST DISC 3 3D. FLD NOM PIELD NAME

ก9 $1950 \quad 1958$

2255500 AMBASSADOR (MISSISSIPPI LIME)

\begin{tabular}{|c|c|c|}
\hline DEPTH & GRAVITY & СОНОL. \\
\hline 6,080 & 41.0 & \\
\hline DEPTH & GRAVITY & corol. \\
\hline 6,493 & 41.0 & \\
\hline$\therefore$ & & \\
\hline YEAR & & $\begin{array}{l}\text { NOL-RELIS } \\
\text { YLOA IIFT }\end{array}$ \\
\hline $\begin{array}{l}1965 \\
1968 \\
1967 \\
1966\end{array}$ & & $\begin{array}{ll}0.0 & 0.2 \\
0.0 & 1.0 \\
0.0 & 1.0 \\
0.0 & 0.9\end{array}$ \\
\hline
\end{tabular}

861,399

$\operatorname{coNTY}$

CIAY

CONNTY

CLAY

536,549
OIL-PRD E/RD

$22 \quad 0.4$

$1409 \quad 3.9$

2491 6.8

$\begin{array}{ll}2491 & 6.8 \\ 3208 & 9.6\end{array}$

DEPTH GRAVITI

6,454

48.0

COHC1. PROD. OF FIELD

467,888

COUNTY

CIAY

DEPTE

GRAV ITY

COUUL. PROD. OP FIEID

CCONTY

5,954

42.0

437,165

CLAY 
ABANDONED ONSHORE OII. FIELDS WITA OVER 250,000 BBL. COAOLAT IVE FRODUCTION

DST DISC ABD. PID NOI PIQLD . NABE

09194119563082500 ANTELOPB (UISSISSIPPI LIME)

DST DISC ABD. PLD NOH PIELD NAHE

O9 $19501974 \quad 481500$ ACAE (HISSISSIPP I LIEE)

DST DISC BBD. PID NOH FIELD NAEE

O9 1953196936126500 GONAN (CADDO, LOHER)

\begin{tabular}{|c|c|c|c|c|}
\hline DEPTH & GRAVIT Y & conol. & PROD. OP PIELD & CoONTY:- \\
\hline 5,670 & 38.0 & & 379,458 & CLAY \\
\hline DEPTH & GRAVITY & COMOL. & PROD. OP PIELD & CCOATY \\
\hline 5,780 & 41.0 & & 350,138 & CLAY \\
\hline
\end{tabular}

$\begin{array}{lll}\text { YEAR } & \text { NOH-甘ELIS } \\ & \text { FLOP } & \text { IIFT } \\ 1974 & & \\ 1973 & 0.0 & 0.6 \\ 1972 & 0.0 & 1.0 \\ 1971 & 0.0 & 1.0 \\ 1970 & 0.0 & 1.8 \\ 1969 & 0.3 & 1.8 \\ 1968 & 0.0 & 2.0 \\ 1967 & 0.0 & 2.0 \\ 1966 & 0.0 & 2.0 \\ 1968 & 0.0 & 2.0\end{array}$

$\begin{array}{rr}\text { OIL-PRD } & E / R D \\ & \\ 1729 & 8.1 \\ 3409 & 9.3 \\ 4057 & 11.1 \\ 6314 & 9.4 \\ 6576 & 9.0 \\ 6551 & 9.0 \\ 5539 & 7.6 \\ 5233 & 7.2 \\ 6120 & 8.4\end{array}$

GA S-PRD

DEPTH GRAVITY

CUROI. PROD. OP FIELD.

$\operatorname{codNTY}$

5,958

43.0

346,316

CL A.I

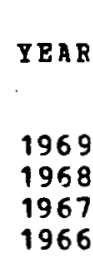

NOH-NELIS

OIL-PRD $E / R D$

GAS-PRD

1969

1958
1967

1966
890.3

$\begin{array}{rl}1453 & 2.0 \\ 2802 & 3.8\end{array}$

28023.8

$5314 \quad 4.9$ 
ARA NDONED ONSHORE OIL FJEIDS VITH OVER 250,000 BBL. COMOLATIVE PRODOCTICN

DST DISC ABD. PID NTM FTELD NAME

$09 \quad 19551968$
DE P TH

40478500 HENRIETTA, NORTH (CONGLOFERATE) 6,226
GRAVITY

43.0
COEOL. FROD. OF FIFLD

270,790

DEPTH GRAVITY

4,582
COHEI. PROD. OF PIELD

$266,8.89$
091953196913838500 BDRNS-MIDRAY (STRANN)
NOM-RELIS PLOD LIPT

$0.0 \quad 0.7$

$0.0 \quad 2.0$

42.0

\begin{tabular}{lll} 
IEAR & \multicolumn{2}{c}{ NOM-RELIS } \\
& PLON LIPT \\
1968 & & \\
1967 & 0.0 & 0.7 \\
1966 & 0.0 & 2.0 \\
& 0.0 & 2.0
\end{tabular}

\begin{tabular}{|c|c|c|}
\hline Y E AR & $\begin{array}{l}\text { NOM-D } \\
\text { PLOR }\end{array}$ & $\begin{array}{l}\text { IELI } \\
\text { LIF }\end{array}$ \\
\hline $\begin{array}{l}1969 \\
1968 \\
1967 \\
1966\end{array}$ & $\begin{array}{l}0.0 \\
0.0 \\
0.0 \\
0.0\end{array}$ & $\begin{array}{l}2 . \\
5 . \\
5 . \\
5 .\end{array}$ \\
\hline
\end{tabular}

CODNTY

CI. A Y 
ABA NDONED ONSHORE OIL PIEIDS ITH OVER 250,000 BBL. COBULATIVE PRODUCTION

DST DISC ABD. FLD NOM FIELD NAME

OO 1955196855361500 NEHPORT, NH. (BRYSON)

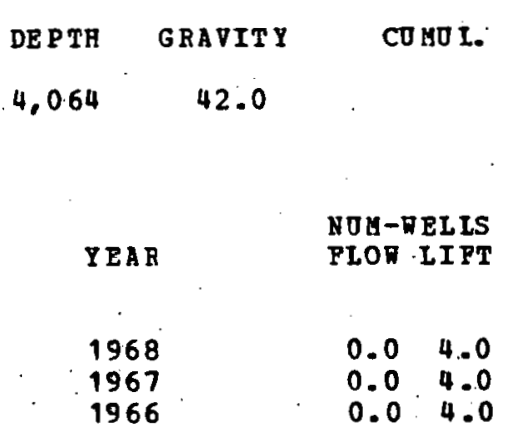

14 PIELD(S)
CONNTY

CLAY

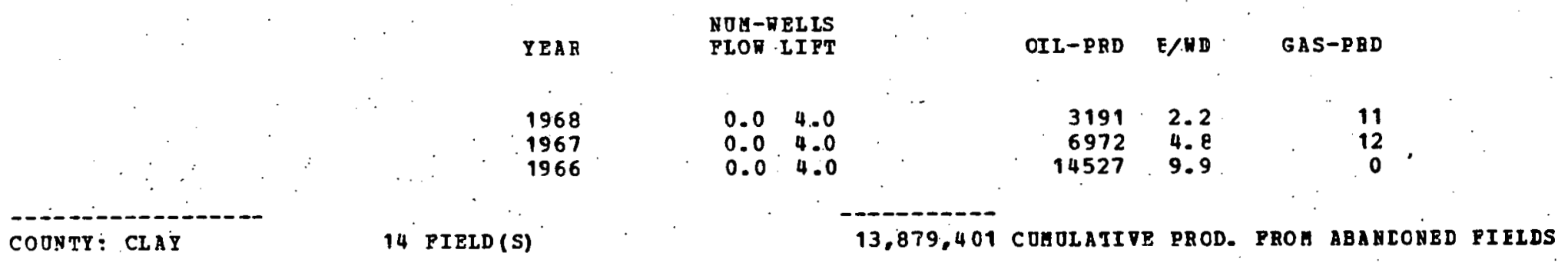


ABANDONED ONSHORE OIS FIELDS WITH OVER 250,000 BBI. COMULATIVE PRODOCTICN

DST DTSE ARD. FID NIM PIELD NAME

$09 \quad 19411962 \quad 8261500$ 9INDEL (ELIENBURERR)

DST DISC ABD. PLD NMM PIEID NAME.

$09 \quad 19571972.98505333$ \#OODBINE (STRAWN 3700)

$\begin{array}{ccccc}\text { DEPTH } & \text { GRAVITY } & \text { COMCL. PROD.. OP FIELD } & \text { CODRTY } \\ 3,200 & 35.2 & & 339.338 & \text { COORE } \\ & & & & \\ \text { DEPTE } & \text { GRAVITI } & \text { CEMOL. PROD. OP FIELD } & \text { CODRTY } \\ 3.745 & 26.0 & 251,758 & \text { COORE }\end{array}$

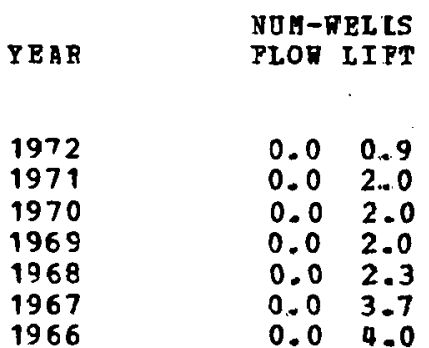

2 PIELD (S)

$\begin{array}{rrr}\text { OII-PRD } & \text { E/RD } & \text { GAS-FBD } \\ & & \\ 1376 & 0.0 & 5 \\ 1330 & 1.9 & 170 \\ 2416 & 3.3 & 123 \\ 3825 & 4.7 & 120 \\ 6996 & 5.2 & 192 \\ 8263 & 5.7 & 353\end{array}$

591,096 COHOLATIVE PRCD. PROH ABANEONBE PIELDS 
AFANDONED ONSHORE OIL PIELDS NITH OVER 250,000 BBL. COHULATIVE PRODUCTICA

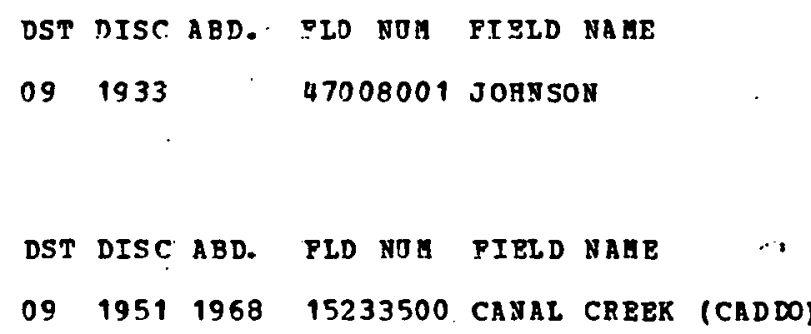

$091951 \quad 1968 \quad 15233500$. CANAL CREEK (CADDO)

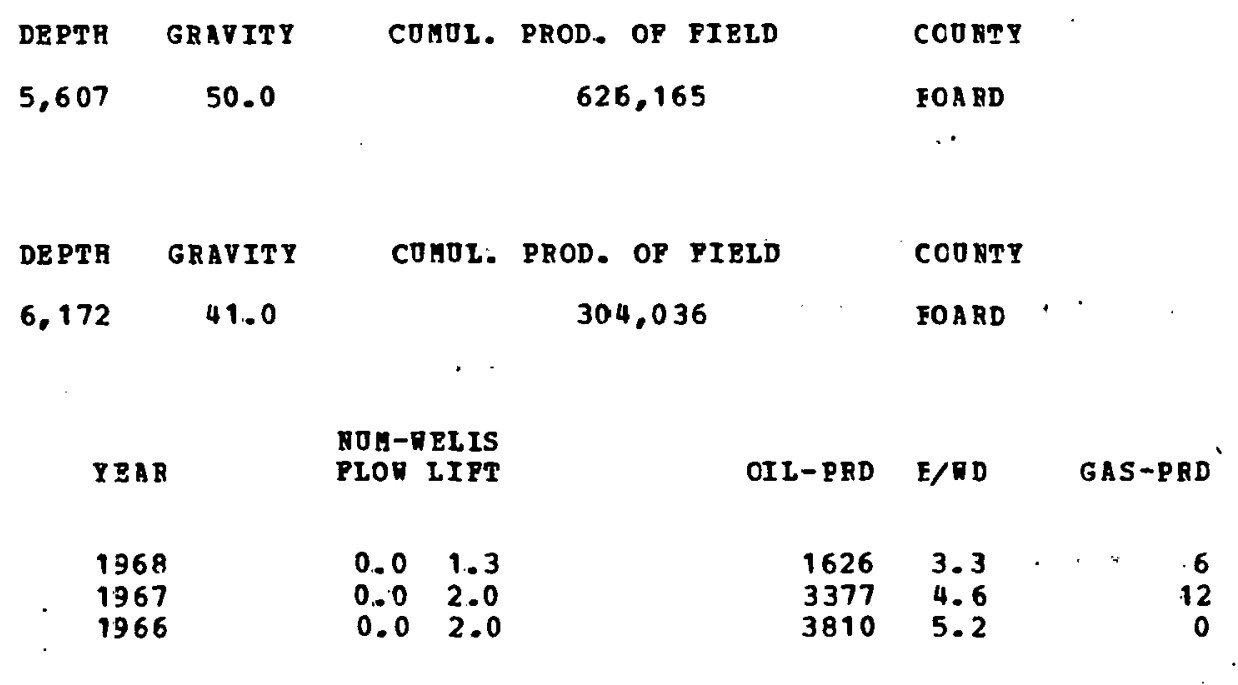

2 FIRID (S) 
ABANDOHED ONSHORE OIZ PIELDS ITH OVER 250,0.00 BBL, COHOLATIVE FRODUCTICE

DST DISC ABD. PLD ROA PIELD NAHE

O9 $1951197559802500 \cdot$ MCAILLAN (OII CREER SAND)

DST DISC ABD. PLD MU PIELD NAHB

091951197363706400 EJLDER (ELLENBOR GER)

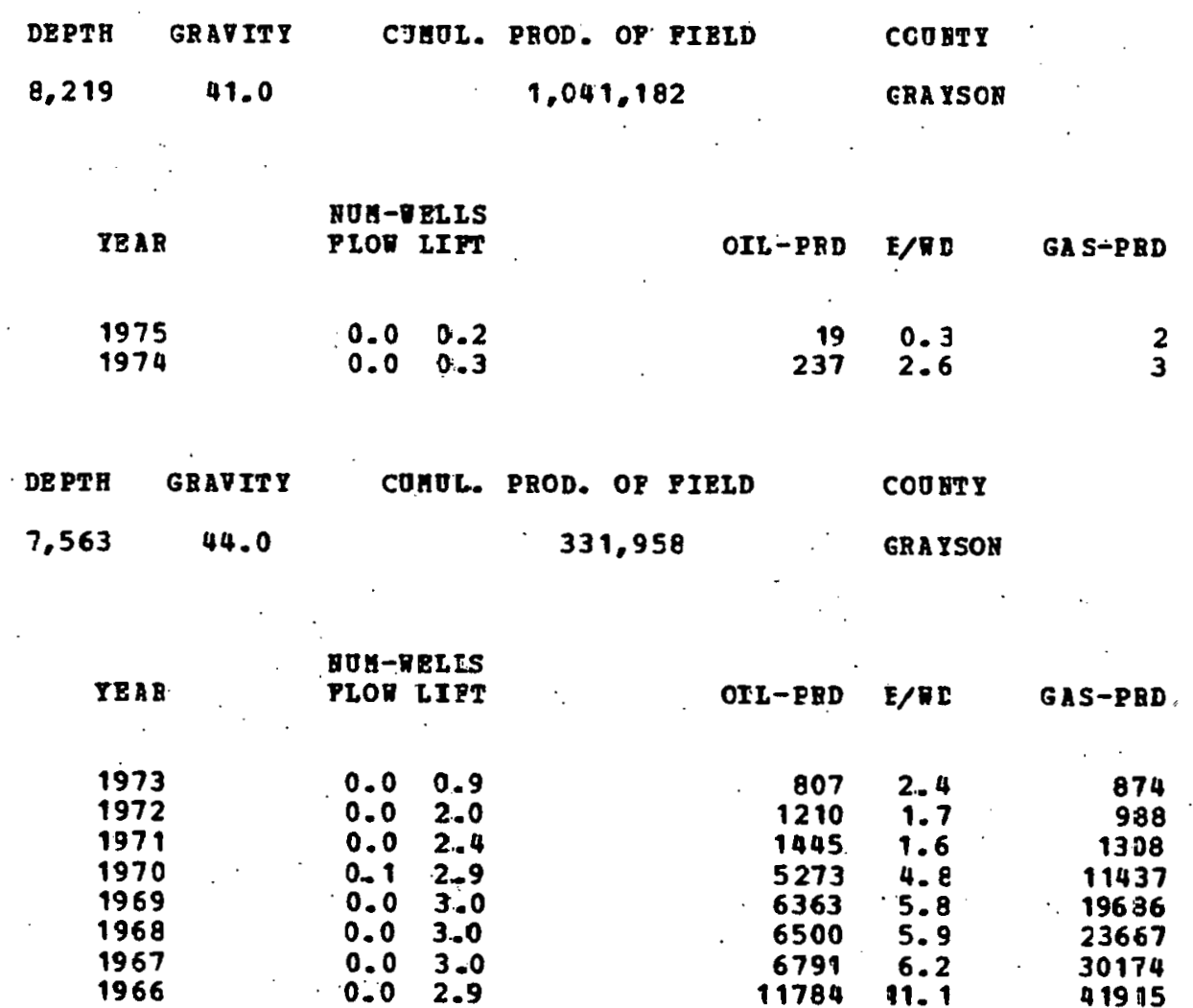


ABANDONED ONSHORE OIL FIELDS VITE OVER 250,000 BBL. COUULATIVE PRODOCTICN

DST DISC ABD. FLD NIM FIELD NAME

$09 \quad 1954 \quad 1974 \quad 83162510 \quad$ SHERHAN (PENNSYLVANIAN)

DE P TH
8,542

$Y 3 A$
1974
1973
1972
1971
1970
1969
1968
1967
1966

DST DISC ABD: PID NOY PIELD NAME

$0919561972 \quad 71239166$ PHILPICK (RIVERS IDE)

5,008

$\begin{array}{cccc}\text { GRAVITI } & \text { CUMDL. } & \text { PROD. OF FIELD } & \text { COONTY } \\ 37.5 & 287.793 & \text { GRAYSON }\end{array}$

\section{NOM-HELIS \\ PLOW LIPT}

CIL-PRD E/RD

GAS-PRD

$\begin{array}{ll}0.3 & 0.4 \\ 0.8 & 0.9 \\ 1.1 & 0.9 \\ 1.0 & 1.0 \\ 1.0 & 0.6 \\ 1.0 & 0.0 \\ 1.0 & 0.0 \\ 1.0 & 0.0 \\ 1.0 & 0.0\end{array}$

$\begin{array}{rrr}321 & 1.3 & 717 \\ 809 & 1.3 & 29339 \\ 19.09 & 2.6 & 68617 \\ 2621 & 3.6 & 80133 \\ 3115 & 5.4 & 82447 \\ 1530 & 4.2 & 84913 \\ 1179 & 3.2 & 69908 \\ 1850 & 5.1 & 103156 \\ 3135 & 8.6 & 139290\end{array}$

\section{DEPTE: GRAVITY}

COMUL. PROD. OF FIELD

COUNTY

34.0

271,469

GRA YSON

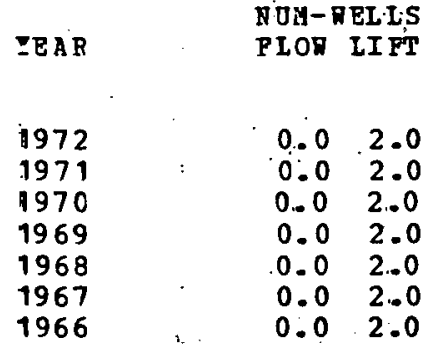

$\begin{array}{rrr}\text { OIL-PRD } & \text { E/RD } & \text { GAS-PRD } \\ & & \\ 814 & 1.1 & 12 \\ 1217 & 1.7 & \therefore \\ 1745 & 2.4 & 12 \\ 2258 & 3.1 & 12 \\ 2716 & 3.7 & 12 \\ 5325 & 7.3 & 360 \\ 13326 & 18.3 & 56635\end{array}$


ABANDONED OKS RORE OII PIELDS ITR OVER 250,000 BRL. CUYULATI PE PRODUCTIOA

DST DISC ABD. PID NTY FIELD RAEE

091963197638678166 HANDY, SE. $(\mathrm{H}-1)$

DEPTH GRAVITI

40.3

Y E A R

1976

1975

1974

1973

1972

1971

1970

1969

1968

1967

1966

COONTI: GRAYSON
5 PIELD (S)
CORI I. PROD. OF FIFID ' COURTY

251,584

GRA YSON

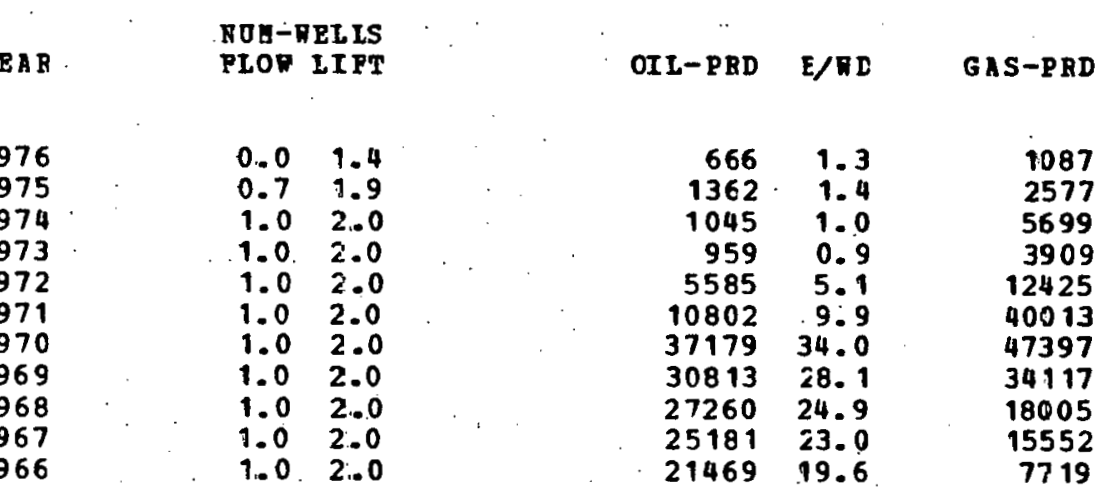


ABA BDONED OHSHORE OII PIBLDS ITH OVER 250,000 BBL. COUULATIVE PRODOCTICX

DST DISC ABD. FLD EOA FIELD MA GE

09 1965. 197689620500 TRRASH (OSAGE)

$\begin{array}{ccccc}\text { DEPTH } & \text { GRAVITY } & \text { CONOL. PROD. OP PIELD } & \text { CCORTY } \\ 8,880 & 49.8 & 1,433,519 & \text { EAREEHAS }\end{array}$

$\begin{array}{rrrrr}\text { ROH-RELIS } & & \\ \text { FLOR LIFI } & \text { OIL-PRD } & \text { E/RD } & \text { GAS-PRD } \\ & & & & \\ 1.7 & 0.0 & 38992 & 64.1 & 136144 \\ 2.3 & 0.0 & 94598 & 111.1 & 264126 \\ 3.0 & 0.0 & 125822 & 114.9 & 355738 \\ 3.5 & 0.0 & 100765 & 78.9 & 319294 \\ 5.0 & 0.0 & 86092 & 47.2 & 344804 \\ 5.0 & 0.0 & 86213 & 47.2 & 425325 \\ 5.0 & 0.0 & 95800 & 52.5 & 492054 \\ 5.0 & 0.0 & 110913 & 60.8 & 743650 \\ 5.0 & 0.0 & 126721 & 69.4 & 727426 \\ 5.9 & 0.0 & 276091 & 127.8 & 970258 \\ 4.9 & 0.0 & 281595 & 156.9 & 557644 \\ & & & & \end{array}$

COUHTY: GARDEQAN

1 PIELD (S) 
ABANDONED ONSHOFE OLL PIELDS NITH OVER 250,000 BBE. COAOLATIVE PRCDOCTICH

DST DISC ABD. PLD NOM PIRLD MAHE

$091951 \quad 197595180666$ MARD-HCCOLLODGR (STRAWY)

DST DISC ABD. FLD ROA FIELD NAME

$0919511964 \quad 17508666$ CHRRRYXIRK (STRADI)

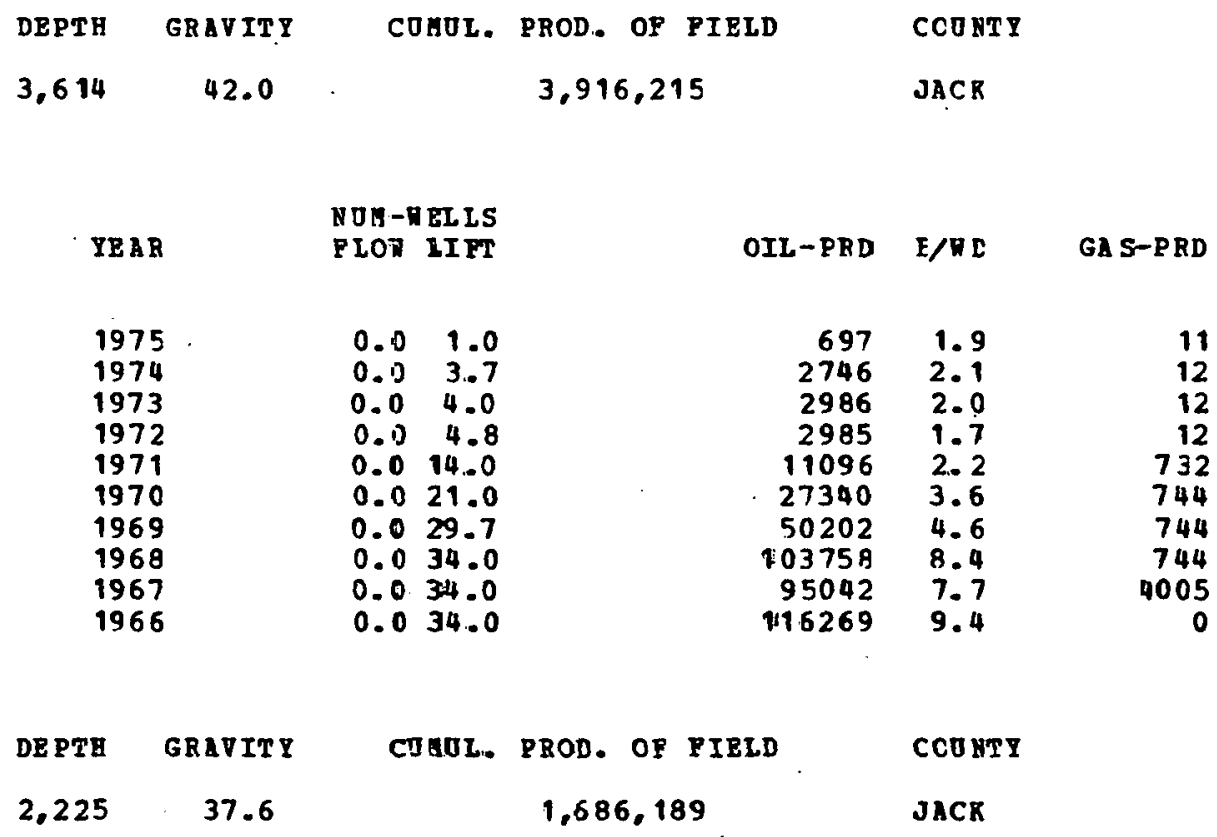


ABAMDONED ONSHORE OIL PIELDS MITH OVER 250,000 BBL. CUMULATIVE FROCUCT ION

DST DIST, AAD, FLD MITM PIELD NAME

$09 \quad 1952$

16107498 CART-HAG (CONGLOBERATE OPPER)

DST DISC ABD. FLD NJH PIELD NBME

$0919511969 \quad 88963666$ TEX-AM (STRARN)

DST DISC ABD. PLD NOM FIRL VIARE

$09 \quad 1954 \quad 1969 \quad 5263500$ BALL (STRARH, IPPBR)

$\begin{array}{cccc}\text { DEPTH } & \text { GRAVITY } & \text { COROL. PROD. OP PIELD } & \text { COONTY } \\ 5,158 & 42.0 & 1,109,823 & \text { JACK } \\ & & & \\ \text { DEPTH } & \text { GRAVITY } & \text { CONOL. PROD. OP PIRLD } & \text { CCORTP } \\ 3,035 & 40.0 & 726,447 & \text { JACK }\end{array}$

\begin{tabular}{|c|c|c|c|c|}
\hline IE AR & $\begin{array}{l}\text { NOA-RELIS } \\
\text { PLOR II TT }\end{array}$ & OIL -PRD & $E / R D$ & GA S-P RD \\
\hline $\begin{array}{l}1969 \\
1968 \\
1967 \\
1966\end{array}$ & $\begin{array}{ll}0.0 & 2.3 \\
0.0 & 7.8 \\
0.0 & 8.0 \\
0.0 & 8.0\end{array}$ & $\begin{array}{r}862 \\
5203 \\
6325 \\
9058\end{array}$ & $\begin{array}{l}1.0 \\
1.8 \\
2.2 \\
3.1\end{array}$ & $\begin{array}{r}6 \\
24 \\
24 \\
0\end{array}$ \\
\hline
\end{tabular}

DEPTH GRAVITY CONOL. PROD. OP PIBLD CCOATY

$3,020 \quad 40.0$

636,389

JACK

\begin{tabular}{lrr} 
YEAR & \multicolumn{2}{l}{ NUS-DELIS } \\
& PLOR LIPT \\
& & \\
1969 & 0.0 & 0.7 \\
1968 & 0.0 & 2.0 \\
1967 & 0.0 & 2.0 \\
1966 & 0.0 & 10.7
\end{tabular}

GAS-PBD

$\begin{array}{rr}0.0 & 2.0 \\ 0.0 & 2.0 \\ 0.0 & 10.7\end{array}$

$0.0 \quad 10.7$

OIL-PRD E/RD

3
12
12
0 
ABANDONED ONSHORE OIL PIELDS ITI OVRR 250,000 BEL. COAOLATIVE PRODOCTICN

DST DISC ABD. PLO NRY FIELD RAME

OO 1952197125892333 DODGS, E. (ANTELOPE STSARN)

$\begin{array}{ccccc}\text { DEPIA } & \text { GRAVITY } & \text { CONOL. PROD. OF EIELD } & \text { COONTY } \\ 3.244 & 39.0 & & 306,468 & \text { JACK }\end{array}$

Y E A B

NOA-REIS

PLOR IIFT

1971

1970

1969

1968

1967

1966

$\begin{array}{ll}0.0 & 4.0 \\ 0.0 & 4.0 \\ 0.0 & 4.0 \\ 0.0 & 4.0 \\ 0.0 & 4.0 \\ 0.0 & 4.0\end{array}$

DEPTH

GRAVITY

4,514

41.0

CO SO 1. EROD. OP FIELD

299.344

CII-PRD E/RD

GAS-PBD

$1521 \quad 1.0$

$2.325 \quad 1.6$

$3209 \quad 2.2$

$3414 \quad 2.3$

$3998 \quad 2.7$

41292.8

COONTY

JACR

YEAR

NDA-VBLIS

PIOA IIFT

OIL-PBD E/RD

GA S-PRD

1968

1967

$0.0 \quad 0.3$

$\begin{array}{rr}842 & 9.2 \\ 827 & 27.2\end{array}$

82727.2

7 PIELD (S) 
ABANDONED ONSHORE OIL PIELDS RITG OVER 250,000 BBL. COUULATIVE PRODOCTICH

DST DISC ABD. FLD NTM PIELD NAME

$09 \quad 1957 \quad 1972 \quad 45796500$ JARVIS : (TANNEHILL)

DST DISC ABD. PLD NOM PIELD NAME

$0919571973 \cdot 50007333$ KNOX CITY (TARERIII)

$\begin{array}{ccccc}\text { LEPTH } & \text { GRAVITI } & \text { CUNOL. PROD. OP PIELD } & \text { CCONTY } \\ 2.067 & 35.0 & 518.184 & & \text { KNOX }\end{array}$

YSAR

NOH-RELIS

OIL-PRD E/RD GAS-PRD

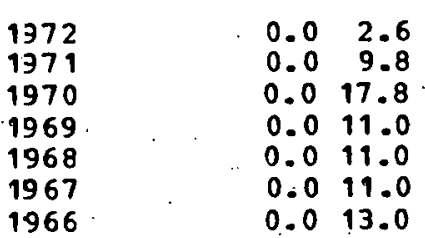

13111.4

$\begin{array}{rr}6809 & 1.9\end{array}$

$35535 \quad 5.5$

7102017.7

6460976.

28657
11284

.

.013 .0

7.1
2.4

COONTY

DEPT!̣

GRAVIT 1

COEOL. PROD. OF FIELD

309.479

RYOX

2,296

38.0

.

Kro

\begin{tabular}{crr} 
YEAR & \multicolumn{2}{c}{ NOH-WELIS } \\
PLON IIFT \\
1973 & & \\
1972 & 0.0 & 0.3 \\
1971 & 0.0 & 2.8 \\
1970 & 0.0 & 3.0 \\
1969 & 0.0 & 3.8 \\
1968 & 0.0 & 9.0 \\
1967 & 0.0 & 12.0 \\
1966 & 0.0 & 12.0 \\
& 0.0 & 12.0
\end{tabular}

GAS-PRD

0.7

0.9
2.2

2. 2

3. 3

2. 1

1.6

2. 1

2
10
12
12
12
12
12
0


ABANDONED ONSHORE OEL FIELDS WITH OVER 250,000 BBL. COMULATIVE PRODUCTICN

DST DISC ABD. FLD NOM FIELD NAME

091956197265614500 NOBA (STRAHN)

\begin{tabular}{|c|c|c|}
\hline DEP TH & GRAVIT Y & I סע ס ב \\
\hline 4,868 & 38.6 & \\
\hline & & NOM-RELIS \\
\hline IEAR & & FIOQ IIPT \\
\hline $\begin{array}{r}1972 \\
1968 \\
+\quad 1967 \\
1966\end{array}$ & & $\begin{array}{ll}0.0 & 0.1 \\
0.0 & -1.7 \\
0.0 & 2.2 \\
0.0 & : 4.0\end{array}$ \\
\hline
\end{tabular}

3 PIELD (S)

$\begin{array}{rrrr}\text { OII-PED } & \text { E/HI } & \text { GAS-PRD } \\ & & & \\ & & \\ 477 & 15.7 & & 0 \\ 1532 & 2.5 & 18 \\ 3120 & 4.3 & + & 219 \\ 3818 & 2.6 & + & 0\end{array}$

1,086,852 CUMOIATIVE PROD. FROM ABANDONED FIELDS 
ABAYDONED OBSHORE OIL PIEIDS RITE OVER 250,000 BBL. COUULATIPE ERODUCTION

DST DISC ABD. PLD RIA FIELD RAHE

$09 \quad 1950 \quad 1967 \quad 48916500$ KERNEDY (CORGLOKERATE)

DST DISC ARD. PID NOM PIELD NALE

09. $19501970 \quad 2440333 \times 18-50$ ? (CONGLCAERATB)

$\begin{array}{ccccc}\text { DEPTE } & \text { GRAVITY } & \text { COEOL. FROD. OP EIELD } & \text { COUHTY } \\ 6,491 & 40.0 & & 1,306,934 & \text { NOHTAGOB }\end{array}$

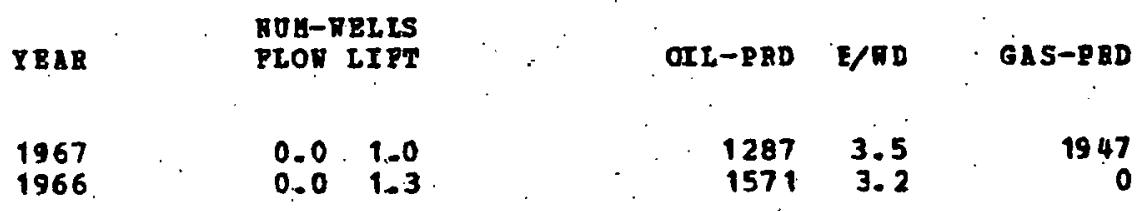

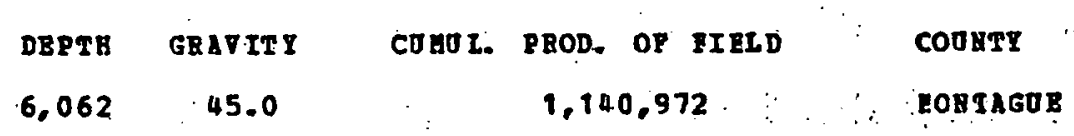

\begin{tabular}{|c|c|}
\hline YEAR- & $\begin{array}{l}\text { NDU-DELLS } \\
\text { FLOQ LIPX }\end{array}$ \\
\hline $\begin{array}{l}1970 \\
1969 \\
1968 \\
1967 \\
1966\end{array}$ & $\begin{array}{rr}0.0 & 1.6 \\
0.0 & 3.0 \\
0.0 & 3.2 \\
0.0 & 9.0 \\
0.0 & 11.0\end{array}$ \\
\hline
\end{tabular}


APANDONED ONSHORE OII. FIIIDS VITA ORER 250,000 BBL. COMULATIVR PRODOCTICN

DST DISC ABD. FLD NTY FIBLD NAME

$091950 \quad 1966 \quad 10988952$ 9ONIE (VIOLA LIEESTONE)

00 195719693574333 ARIES, REST (CADDO)

DST DISC ABD. FLD NUM PIBLD NAME

$0919421969 \quad 80552001$ SARDRRS

$\begin{array}{ccccc}\text { DEPTH } & \text { GRAVITY } & \text { COMOL. } & \text { PROD. OP FIELD } & \text { CONNTY } \\ 6,470 & 43.3 & & 677,990 & \text { MONTAGUE }\end{array}$

YEAR

NOH-TELIS

1966

0.01 .2

CIL-PRD E/RD

GAS-PBD

$1060 \quad 2.5$

0

DEPTA GRAPIT

CU⿴囗十. PHOD. OP FIELD

COONTY

6,100

42.0

659,421

MONTAGOE

$\begin{array}{lrr} & \text { MOA-NELLS } \\ \text { YEAR } & \text { PLOR ITPT } \\ & & \\ 1969 & 0.0 & 5.8 \\ 1968 & 0.0 & 8.4 \\ 1967 & 2.7 & 6.3 \\ 1966 & 3.1 & 6.0\end{array}$

$\begin{array}{rrr}\text { OIL-PRD } & \text { E/NE } & \text { GAS-PAD } \\ & & \\ 2422 & 1.1 & 14717 \\ 5052 & 1.6 & 54626 \\ 8108 & 2.5 & 61759 \\ 12757 & 3.8 & 95713\end{array}$

DEPTH GRAVITY

CUAOL PROD. OP PIELD

COUNTY

5,975

36.8

535,702

MONTAGUE

$\begin{array}{lrrrrr}\text { YEAR } & \begin{array}{c}\text { NOM-RELIS } \\ \text { PLOD LIFT }\end{array} & \text { OIL - PRD } & \text { E/RE } & \text { GAS-PRD } \\ & & & & & \\ 1968 & 0.0 & 0.6 & 604 & 2.8 & 1789 \\ 1967 & 0.0 & 1.0 & 1742 & 4.8 & 4387 \\ 1966 & 0.0 & 1.0 & 1992 & 5.5 & 4435\end{array}$


A BA NDONED ONSHORE OIL FIEIDS IITH OVER 250,000 BBL. COMULATIVE PRODUCTICN

DST DTSC ABD. PLD NOY FIELD NAHE

091960197127144875 BARES (VIOLA IIME)

DST DISC ABD. PLO NOM PIELD NAHE

091952197092436500 ONDERMOOD-RAY (CCRGLOHERATE)

$\begin{array}{cccc}\text { DEPTH } & \text { GRAVITI } & \text { COHOL. FEOD. OF FIELD } & \text { CONATY } \\ 6,295 & 46.8 & 519.627 & \text { MONTAGOE }\end{array}$

\begin{tabular}{|c|c|c|}
\hline Y Q A B & \multicolumn{2}{|c|}{$\begin{array}{l}\text { NOB-RELIS } \\
\text { PLOR LIFT }\end{array}$} \\
\hline $\begin{array}{l}1971 \\
1970 \\
1969 \\
1968 \\
1967 \\
1966\end{array}$ & $\begin{array}{l}3.0 \\
3.0 \\
3.0 \\
4.3 \\
4.9 \\
4.6\end{array}$ & $\begin{array}{l}0.0 \\
0.0 \\
0.0 \\
0.0 \\
0.0 \\
0.0\end{array}$ \\
\hline
\end{tabular}

DEPTH GRAVITY

COMOL. PROD. OP FIELD

COONTI

$6.023 \quad 42.2$

475,399

$\begin{array}{rrr}\text { OII-PRD } & \text { E/RD } & \text { GAS-PRD } \\ & & \\ 765 & 0.7 & 119137 \\ 5254 & 4.8 & 351689 \\ 32024 & 29.2 & 477 \\ 70234 & 44.4 & 12 \\ 2604 & 1.5 & 2 \\ 301 & 0.2 & 19760\end{array}$

\begin{tabular}{lll} 
TEAR & \multicolumn{2}{c}{ ROA-DELIS } \\
PLOT IIFT \\
1970 & & \\
1969 & 0.0 & 0.4 \\
1968 & 0.0 & 3.8 \\
1967 & 0.0 & 2.2 \\
1966 & 0.0 & 1.0 \\
& 0.0 & 1.0
\end{tabular}

OIL-PRD E/RI

GAS-PRD

$\begin{array}{rr}53 & 0.3 \\ 3359 & 2.4 \\ 4883 & 6.2 \\ 1338 & 3.7 \\ 1663 & 4.6\end{array}$

3
52
13
11
0 
A BA NDONED ONSHORE OIZ PIELDS ITTH OVER 250,000 BBL C CUOLATIVE PRODUCTICA

DST DISC ABD. PLD NOY FIELD HAHE

09

79650656 SAI HT J0。

(EON GLOMERATE 6300) 6,582

GRAVIT

40.0

COUUI. FROD. OP PIELD

467,612

OIL-PRD $E / V I$

5703.7

1234.3 .4

$1580 \quad 4.3$

2039

$2750 \quad 3.2$

53154.9

695260

142.0913 .0

1967

1966

DST DISC ABD. PLD NDE PIELD NAHE

$0919501967 \quad 10105001$ BOEDEK ER

DEPTE GRAVITY

6,794

43.9
COJOL. PROD. OF PIELD

450,075
GAS-PAD

$$
\begin{array}{r}
10 \\
12 \\
12 \\
12 \\
1327 \\
2862 \\
4306 \\
6265 \\
5835
\end{array}
$$

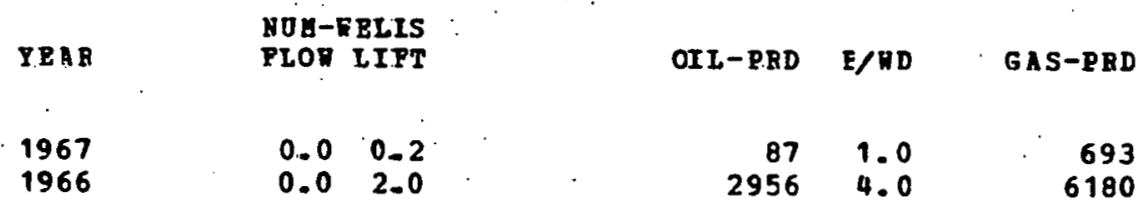


ABA MDOBED OXSHORE OII FIELDS WTH OVER 250,000 BBL. COEOLATIVB PRODDCTICB

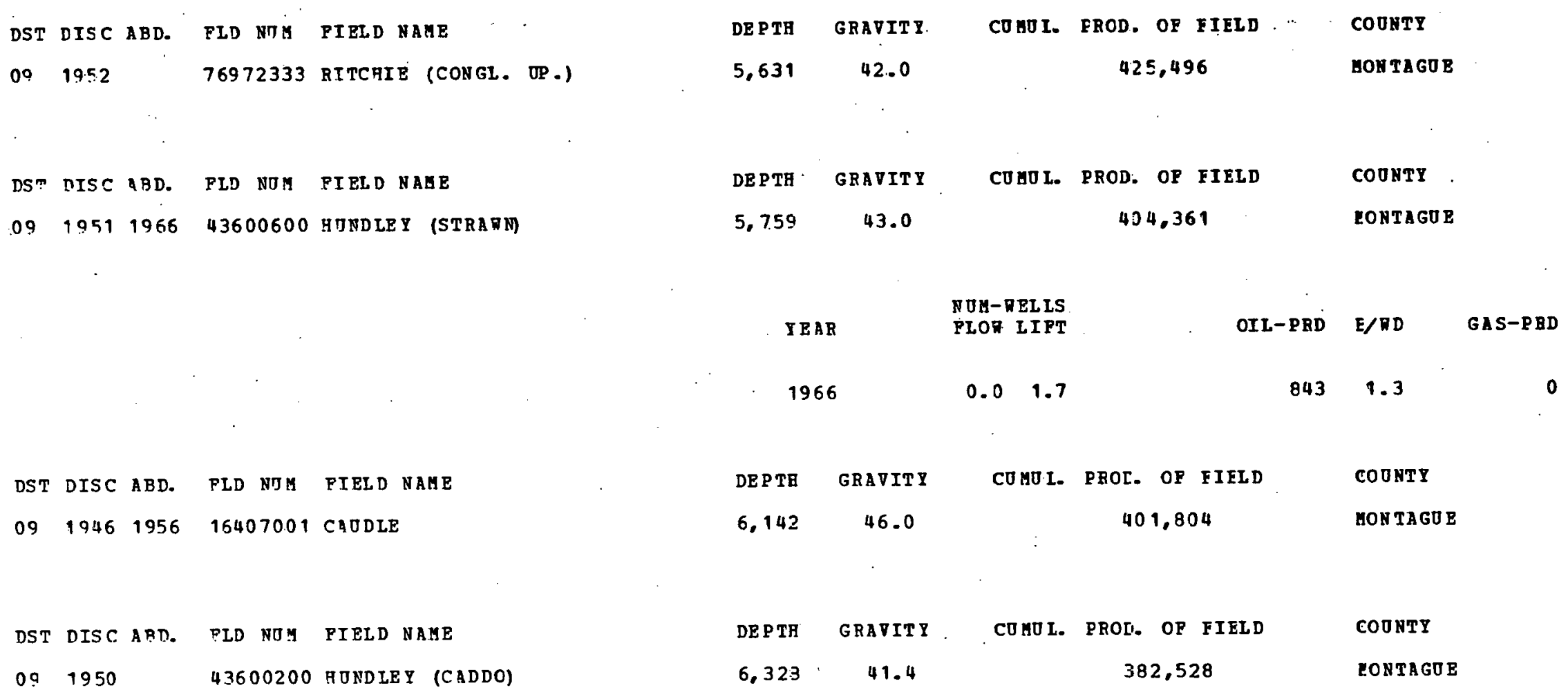


ABANDONED ONSHORE OIL FIEIDS ITH OVER 250,000 BBI. CUMULATIVE PRODUCTICA

DST DISC ABD. FLD NOM FIELD NAME

OO $1941958 \quad 18977001$ CIINGINGSHTTR

DST DISC ABD. FLD NIT FIELD NAME

091952197327144500 EANES (CONGLOMERATE)

\begin{tabular}{|c|c|c|}
\hline DEPTH & GRAVITY & Co $20 \mathrm{I}$. \\
\hline 6,131 & 38.5 & \\
\hline DEP TB & GRAVITY & CU: 1. \\
\hline 5,854 & 42.0 & \\
\hline YEAR & & $\begin{array}{l}\text { YOH-DELIS } \\
\text { PLOR IIPT }\end{array}$ \\
\hline $\begin{array}{l}1973 \\
1972 \\
1971 \\
1970 \\
1969 \\
1968 \\
1967 \\
1966\end{array}$ & & $\begin{array}{rr}0.1 & 0.0 \\
1.5 & 0.0 \\
6.0 & 0.0 \\
6.0 & 0.0 \\
6.0 & 0.0 \\
8.8 & 11.6 \\
10.0 & 0.0 \\
10.0 & 1.0\end{array}$ \\
\hline
\end{tabular}

DEPTH GRAVITY

5,338

43.9

CJ UEI. PROD. OF FIELD

289,234

OII-PRD E/HE GAS-PBD

$\begin{array}{rr}31 & 1.0 \\ 320 & 0.6 \\ 1018 & 0.5 \\ 6091 & 2.8 \\ 9567 & 4.4 \\ 13731 & 1.8 \\ 41719 & 11.4 \\ 74275 & 20.3\end{array}$

COONTY

NONTAGUE

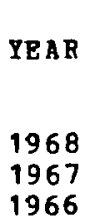

16 FIELD (S)
COUNTY

MONTAGUE

COONTY

NONTAGOE

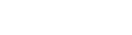

$\begin{array}{rr}1.0 & 372 \\ 0.6 & 4937\end{array}$

$0.5 \quad 26068$

161891

195095

$5 \approx 1875$

1533145

1157025

\begin{tabular}{|c|c|c|c|}
\hline PLON LI FT & $O I I-P R D$ & E/H D & GA S-P RD \\
\hline $\begin{array}{ll}0.0 & 2.0 \\
0.0 & 2.0 \\
0.0 & 2.0\end{array}$ & $\begin{array}{l}4233 \\
6311 \\
8018\end{array}$ & $\begin{array}{r}5.8 \\
8.6 \\
11.0\end{array}$ & $\begin{array}{l}22710 \\
34340 \\
35152\end{array}$ \\
\hline
\end{tabular}

O9 1945 1968 56808001 MAGUIRE

COTNTY: MONTAGJE 
ABANDONED ONSHORE OIL PIELDS HITH OVER 250,000 BBL. COHOLATIVE PROCOCTION

DST DTSC ABD. PLD NOM PIELD NAHB

091948196738553200 RAMMON (CANYON SAND)

DST DISC A BD. PID NOM PIBLD NAUE

091949197038553800 คA HMON (VOGTSBERGER)

$\begin{array}{ccccc}\text { DEPTH } & \text { GRAVITY } & \text { COMOL. } & \text { PROD. OP FIELD } & \text { COUNTY } \\ 2,964 & 42.0 & 1,043,053 & \text { NICHITA }\end{array}$

YEAR

NDV-RELIS

OII-PRD E/RD

GAS-PRD

1967

1966

$\begin{array}{rr}0.0 & 9.8 \\ 0.0 & 13.0\end{array}$

$\begin{array}{rr}1274 & 0.4 \\ 991 & 0.2\end{array}$

8
1094

DEPTH GRAVITY

CO MO 1. FROD, OP FI ELD

COUNTY

4. 859

42.0

$1,010,729$

IICHITA

YEAR

NOM-NELLS

PLOR IIFT

OIL-PRD E/WL

GA S-PRD

1970

1969

1968

1967

1966

$0.0 \quad 5.0$

0.05 .0

$0.0 \quad 6.4$

$0.0 \quad 12.0$

0.012 .0

$6675 \quad 3.7$

$8494 \quad 3.6$

$12810 \quad 2.9$

$17414 \quad 4.0$ 
ABA RDONED JNSHORE OIL PIEIDS RITH OVER 250,000 BBL. COHOLATIVE PRODOCTICA

DST DISC ABD. PLD WOH FIBLD NABB

091952197348712001 REHPHER, PBST
DE P TH
GRAVIT Y
CO MO I.
ROD. OF FIEID
CONNTY
3,906
42.5
928,823
ดIC 日ITA

\begin{tabular}{lll} 
YEAR & \multicolumn{2}{c}{ NOEL-REIIS } \\
PLON & EIPT \\
& & \\
1973 & 0.0 & 5.5 \\
1972 & 0.0 & 6.0 \\
197.1 & 0.0 & 6.0 \\
1970 & 0.0 & 6.0 \\
1969 & 0.0 & 6.1 \\
1968 & 0.0 & 6.0 \\
1967 & 0.0 & 6.0 \\
1966 & 0.0 & 6.0
\end{tabular}

$\begin{array}{rr}\text { OII-PRD } & \text { E/RD } \\ & \\ 1413 & 0.7 \\ 2103 & -1.0 \\ 2911 & 1.3 \\ 3470 & 1.6 \\ 3036 & 1.4 \\ 2888 & 1.3 \\ 3956 & 1.8 \\ 3802 & 1.7\end{array}$

GAS-PBD

12
12
12
12
12
12
12
0

DST DISC ABD. PLD ROM PIELD NA GE

$0919381967 \quad 77594666$ MOCR CROSSI RG (ELLERBORGER)
GRAVIT Y

39.0
COUO 1. PROD. OP FI ELD

788.810
$\operatorname{COONTP}$

nICคITA
IE A R

1967

1966
NOB-RELIS

PIOD II FT

$0.0 \quad 0.4$

$0.0 \quad 5.0$
OII.PRD E/HD

$\begin{array}{rrr}167 & 1.1 & 2 \\ 451 & 1.3 & 0\end{array}$


ABANDONED ONSHORE OIL FIELDS MITA OVER 250,000 B.BL. CUHOLATIVE PRODUCT ION

DST DISC ABD. FLD NIM FTELD NAME

$09 \quad 1941$

39282001 RARROL D

DST DISC ABD. PLD NOM FIELD HA GE

$0919561971^{\circ}$

30215500 PARGO, D. (STRARN CONGLOAERATE)

DEPTH

GRAVITY

45.4

COEOL. PROD. OF PIELD

\begin{tabular}{lll} 
& \multicolumn{2}{c}{ NOH-AELIS } \\
YEAR & PLOR LIFT \\
& & \\
1971 & 0.0 & 1.8 \\
1970 & 0.0 & 5.0 \\
1969 & 0.0 & 5.3 \\
1968 & 0.0 & 7.8 \\
1967 & 0.0 & 7.3 \\
1966 & 0.0 & 6.0
\end{tabular}

DE PTH

GRAVITY

42.0

CUHOL. PROD. OF PIELD

$09 \quad 1947$

30209852 PARGO (ELLENBURG ER)

6,250
474.481

CCọ̣TY

IIC BITA

COONTI

\section{CCO ETY}

IIC BITa

$\begin{array}{rrr}\text { OII - PRD } & \text { E/RD } & \text { GR S-PRD } \\ & & \\ 310 & 0.5 & 197 \\ 488 & 0.3 & 826 \\ 3309 & 1.7 & 4268 \\ 5749 & 2.0 & 4074 \\ 4726 & 1.8 & 5087 \\ 5547 & 2.5 & 12263\end{array}$

324,959

vIC 
ABANDONED ONSHORE OIL FIELDS NITA CVER 250,000 BBL. COUOLATIVE PROEOCTION

DST DISC ABD. PLS NOM FIELD NAME

$09 \quad 1943 \quad 1969 \quad 77925001$ ROGERS-MCCRARY

\begin{abstract}
DEPTH GRAVITT
1,489

39.0
\end{abstract}

COYOL. PROD. OF PIELD

267.660

COONTY

NICHITA

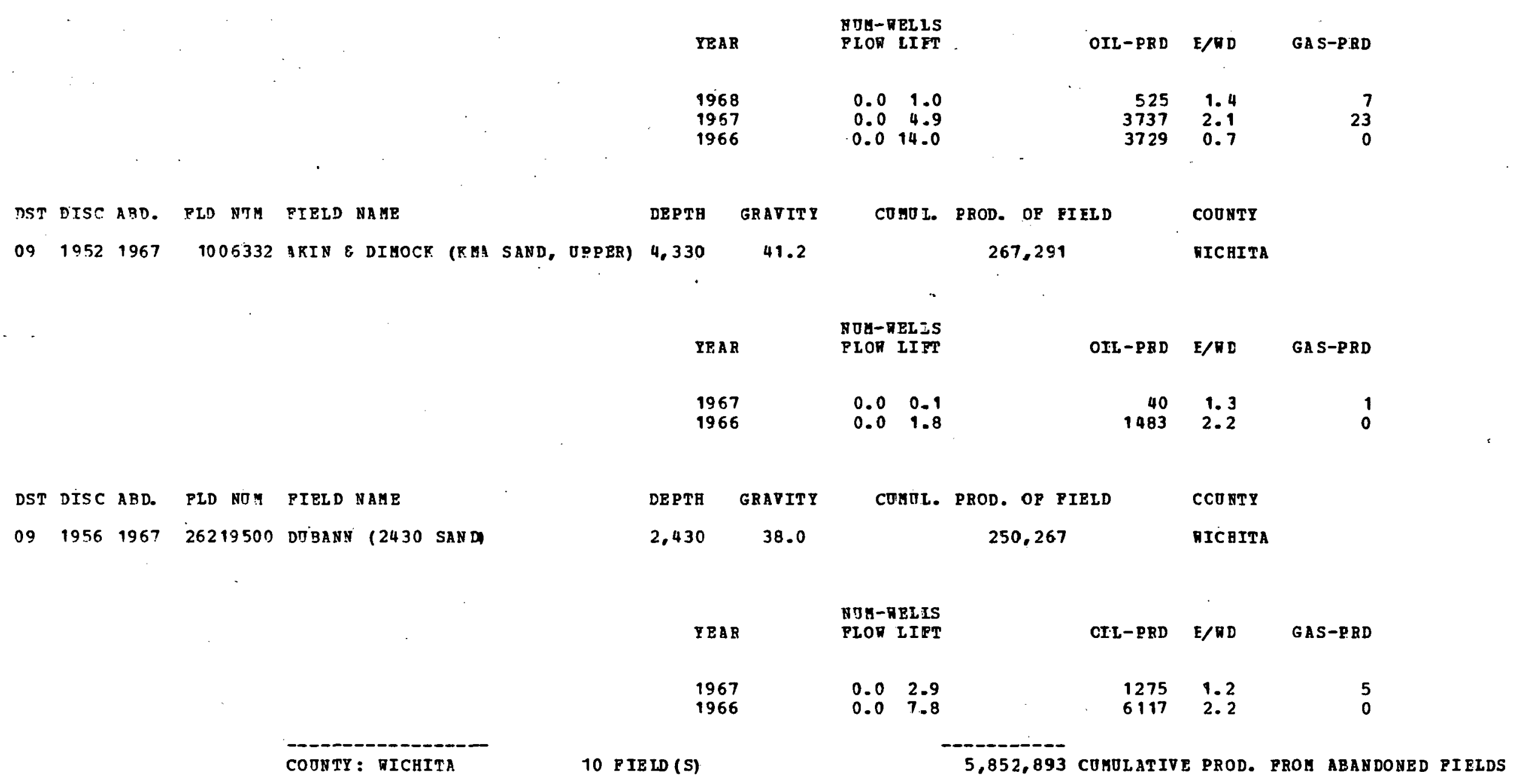


ABANDONED ONSHORE OIL FIELDS RITH OVER 250,000 BBL. COUDLAT IVE FROLUCTICN *

DST DISC ABD. FLD NUM PIELD NAME

$09 \quad 1933$

82450001 SEREIL

DST DISC ABD. PID NOM PTELD NAME

$0919541974 \quad 34076830$ GARVEY (STRANN 3200)

DST DISC ABD. FLD NUM PIELD NAME

$0919411969 \quad 1599001$ ALLAR

\begin{tabular}{|c|c|c|c|c|}
\hline DE P TH & GRAVITY & COMUI. & PROD. OF FIELD & COUNTY \\
\hline 3,800 & 44.0 & & $1,401,955$ & YODNG \\
\hline DEPTH & GRAVIT I & COMU I. & PROD. OF FIELD & COUNTY \\
\hline 3,262 & 41.0 & & $1,126,873$ & ICONG \\
\hline
\end{tabular}

YEAR
1974
1973
1972
1971
1970
1969
1968
1967
1966

NOA-RELIS

FLON LIPT

OIL-PRD E/RD

GA S-PRD

$\begin{array}{ll}0.0 & 1.8 \\ 0.0 & 7.0 \\ 1.7 & 5.3 \\ 0.0 & 7.0 \\ 0.0 & 7.0 \\ 0.0 & 7.0 \\ 0.0 & 7.0 \\ 0.0 & 6.4 \\ 0.0 & 6.0\end{array}$

$733 \quad 1.1$

$4110 \quad 1.6$

$4451 \quad 1.7$

$4786 \quad 1.9$

52362.0

62412.4

$6802 \quad 2.7$

57012.4

1966

$5974 \quad 2.7$

9
53
731
703
875
1251
1383
2126
0

DEPTH

GRAVIT Y

COMOL. EROD. OF FI ELD

COUNTY

2,750

38.0

$1,121,913$

ICONG

\begin{tabular}{ccc} 
& \multicolumn{2}{c}{ NOM-YELIS } \\
YEAR & FLON LIFT \\
& & \\
1969 & 0.0 & 1.5 \\
1968 & 0.0 & 2.5 \\
1967 & 0.2 & 3.0 \\
1966 & 0.0 & 3.0
\end{tabular}

$\begin{array}{rr}\text { OIL - PRD } & \text { E/HD } \\ & \\ 1383 & 2.5 \\ 3374 & 3.7 \\ 5802 & 5.0 \\ 6920 & 6.3\end{array}$

GA S-P RD

9
12
12
0 
ABANDONED ONSHORE OII PIELDS IITH OVER 250,000 BBL. CUBOLATIVE PROLOCTION

DST DISC ARD. PLD NJM FIELD NA UE

0

\author{
(STRANR 3200)
}

DE PTH

3,200

GRAVIT Y

42.0

COHOL. PROD. OP PIELD

C.CUNTY

YEA R

1966

$$
\begin{aligned}
& \text { NUM-RELLS } \\
& \text { PLOW LIFT }
\end{aligned}
$$

0.014 .2

825,761

ICD NG

3,476

GR A IT Y

42.0

CUMUL. PROD, OF. FIELD

742.346

E/M D

14847

2. 9

GA S-P RD

442

\begin{abstract}
$09 \quad 1946$ 488 14332. KENDALI (CADDO)
\end{abstract}

DST DISC ABD. FID NDM FIELD NAME

091941197649961500 KNOX, NORTH (CADDO)
DEPTE GRAVIT Y

$3,950 \quad 42.0$

CLMUL. PROD. OF PIELD

667,354

$\operatorname{CCONTY}$

YOUNG

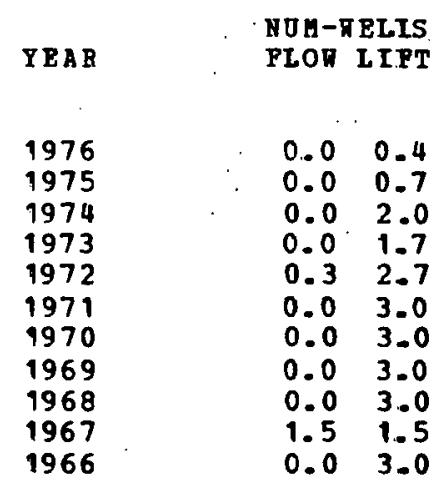

$\begin{array}{rrr}\text { OIL-PRD } & \text { E/WD } & \text { GAS-PRD } \\ & & \\ 258 & 1.7 & 6 \\ 298 & 1.2 & 724 \\ 554 & 0.8 & 1728 \\ 559 & 0.9 & 1440 \\ 692 & 0.7 & 1728 \\ 1215 & 1.1 & 1728 \\ 1503 & 1.4 & 1728 \\ 1102 & 1.0 & 1728 \\ 948 & 0.9 & 1728 \\ 1226 & 1.1 & 3108 \\ 1439 & 1.3 & 5040\end{array}$


A SANDONED ONSHORE OIL FIELDS WITE OVER 250,000 BBL. COHOLATIVE FROLOCTION

DST DTSC ABD. PLD NTM PIELD NAEE

09195519699703500 BLDONT (BOTTRAE).

DST DISC ABD. PID NOM. FIELD NAEE

Og 1942196368313500 PADGTTT (HISSISSIPPI LIHE)

DST DISC ABD. gID NOM PIELD YAME

0919481974,95286750 RARREH (STRAYH)

$\begin{array}{ccccc}\text { DEPTH } & \text { GRAVITY } & \text { CUROL. PRCD. OF PIELD } & \text { COURTY } \\ 2.134 & 41.8 & \ldots & 506,683 & \text { YCOAG }\end{array}$

\begin{tabular}{|c|c|c|c|c|}
\hline YEAR & $\begin{array}{l}\text { ROH-RELIS } \\
\text { PLON LIFT }\end{array}$ & OIL-PBD & E/RC & GA S-PRD \\
\hline $\begin{array}{l}1968 \\
1967 \\
1966\end{array}$ & $\begin{array}{ll}1.5 & 4.5 \\
0.0 & 9.0 \\
0.0 & 9.0\end{array}$ & $\begin{array}{l}1233 \\
1884 \\
4143\end{array}$ & $\begin{array}{l}0.6 \\
0.6 \\
1.3\end{array}$ & $\begin{array}{r}14 \\
24 \\
3036\end{array}$ \\
\hline
\end{tabular}

DEPTH GRATITI COEI. FRCD. OF FIELD COOATY

$4.800 \quad 44.2 \quad 491.890 \quad$ YCONG

DEPTH GRAVITI COMOL. PROD. OF FIELD CCOATY

$\begin{array}{llll}2,912 & 41.0 \quad 382.628 & \text { YCOBG }\end{array}$

\begin{tabular}{|c|c|c|c|c|c|}
\hline YEAB & $\begin{array}{l}\text { Y On- } \\
\text { F LON }\end{array}$ & $\begin{array}{l}\text { ELLS } \\
\text { LIFT }\end{array}$ & OIL-PRD & $E / D D$ & GA S-PRD \\
\hline $\begin{array}{l}1974 \\
1973 \\
1972 \\
1971 \\
1970 \\
1969 \\
1968 \\
1967 \\
1966\end{array}$ & $\begin{array}{l}0.0 \\
0.2 \\
0.0 \\
0.0 \\
0.0 \\
0.0 \\
0.2 \\
0.0 \\
0.0\end{array}$ & $\begin{array}{l}1.2 \\
1.8 \\
2.0 \\
2.0 \\
2.0 \\
2.0 \\
1.8 \\
2.0 \\
2.0\end{array}$ & $\begin{array}{r}134 \\
959 \\
1602 \\
2444 \\
3251 \\
5278 \\
6920 \\
7778 \\
9026\end{array}$ & $\begin{array}{r}0.3 \\
1.3 \\
2.2 \\
3.3 \\
4.5 \\
7.2 \\
9.5 \\
10.7 \\
12.4\end{array}$ & $\begin{array}{r}1261 \\
2520 \\
2455 \\
1740 \\
1740 \\
1740 \\
1740 \\
1596\end{array}$ \\
\hline
\end{tabular}


9 9ANDONED ONSHORE OIL PIELDS ITH OVER 250,000 B9L. COHULATIVE PRODUCTICA

DST DISC ABD. PLD NDY PIELD NAKE

091948197695286250 MARREN (CADDO)

\begin{tabular}{|c|c|c|c|}
\hline DEPTH & GRAVIT Y & \multicolumn{2}{|c|}{ CD } \\
\hline 3,639 & 45.0 & & \\
\hline TEAR & & $\begin{array}{l}\text { ROH-T } \\
\text { PLON }\end{array}$ & $\begin{array}{l}\text { EL IS } \\
\text { LIFT }\end{array}$ \\
\hline $\begin{array}{l}1976 \\
1974 \\
1973 \\
1972 \\
1971 \\
1970 \\
1969 \\
1968 \\
1967 \\
1966\end{array}$ & & $\begin{array}{l}0.0 \\
0.0 \\
0.0 \\
0.0 \\
0.0 \\
0.0 \\
0.0 \\
0.1 \\
0.0 \\
0.0\end{array}$ & $\begin{array}{l}0.2 \\
1.2 \\
2.0 \\
2.3 \\
3.0 \\
3.0 \\
2.7 \\
2.0 \\
2.0 \\
1.0\end{array}$ \\
\hline
\end{tabular}

COONTY

367,422

YOONG
DST DISC ABD. FLD NOM PIELD NABE

091950196846013500 JEAN, REST (STHA DH?)
DEPTH GBAVIT $Y$

3,108

42.0
COHOL. PROD. OP FIELD

364.812
CIL-PRD E/RE

$\begin{array}{rrr}29 & 0.5 & 2 \\ 342 & 0.7 & 1055 \\ 797 & 1.1 & 2520 \\ 1640 & 2.0 & 2372 \\ 1503 & 1.4 & 4125 \\ 2008 & 1.8 & 4920 \\ 4563 & 4.7 & 6900 \\ 3113 & 4.1 & 3932 \\ 1578 & 2.2 & 3425 \\ 2148 & 3.1 & 1838\end{array}$

YEAR

1968

1967

1966

\section{NOL-RELIS}

PLON IIFT

$$
\begin{array}{ll}
0.0 & 2.1 \\
0.0 & 5.0 \\
0.0 & 5.5
\end{array}
$$

\section{OII}

$\begin{array}{llr}1014 & 1.3 & 985 \\ 3402 & 1.9 & 1623 \\ 3353 & 1.7 & 1402\end{array}$


A BA NDONED ONSHORE OIL PIEIDS NTTA OVER 250,000 BBL. COEOLATIVE PRODUCTICH

DST DISC ABD. FLD NOA PIELD NAME

091949

22867166 DALY (CADDO)

OST DISC ABD. PLD NOH PIBLD NAEE

0요 1939

3222001 ARZAE GRAHAH

DST DISC ABD. PLD NTH PIELD HAHE

091954

69384142 MILES-JACRSON (BEND CONGL)

DST DISC ABD. FLD NOI FIELD NAME

D9 $19561974 \quad 41337500$ RIGHTORER (MISSISSIPPIAN)

\begin{tabular}{|c|c|c|c|c|}
\hline DE PTH & GRAVITI & CO I. & EROD. OF FIELD & COONTY \\
\hline 3,856 & $47 . .4$ & $\cdot$ & 355,299 & YOUNG \\
\hline DEP TH & GRAVITY & COHOL. & PROD. OF FIELD & COUNTY \\
\hline 4.000 & 32.0 & & 327,035 & YOOKG \\
\hline DEPTH & GRAVITY & cu no I. & PROD. OF FIELD & COUNTI \\
\hline 5,310 & 43.0 & & 272.772 & ICO NG \\
\hline DEPTH & GRAVITI & COKסL. & PEOD. OF PIELD & $\operatorname{ccosTr}$ \\
\hline 5,160 & 44.0 & & 271,406 & YCO YG \\
\hline
\end{tabular}

IE AR

NOB-RELIS

FION LIPT

OIL-PRD E/RE

GA S-PRD

$1974 \quad 0.0 \quad 0.8$

$1973 \quad 0.01 .0$

0.01 .1

19710.02 .0

$197 \mathrm{C} \quad 0.31 .8$

$1969-1.01 .0$

1.01 .0

$\begin{array}{lll}1967 & 1.1 & 0.9 \\ 1966 & 2.0 & 0.0\end{array}$

$\begin{array}{rrr}701 & 2.6 & 10 \\ 1180 & 3.2 & 12 \\ 1611 & 4.1 & 131 \\ 4601 & 6.3 & 2179 \\ 6168 & 8.4 & 935 \\ 7078 & 9.7 & 792 \\ 7930 & 10.9 & 1294 \\ 13525 & 18.5 & 3280 \\ 13852 & 19.0 & 0\end{array}$

CONNTY: YOONG

DISTRICT 09
15 FIELD (S)

89 FIELDS
9,226,649 COMOLATIVE PROD. FROM ABANDONED FIELDS $60,654,327$ CUMULAIIVE PRDD. PROM ABANCONED FIELDS 
ABARDONFD IONSHORE OIL FIELDS MITH OVER 250,000 BBL. CUGOLATIVE PRODUCT IOA

DST DISC ABD. FLD VII PIELD HAEE

1019541971.75696500 RERH (GRAMTTE DASH)

DEP IA
6,164


$Y B A B$
1971
$197 C$
1969
1968
1967
1966

1 PIELD (S)
COEU L. PROD. OF FIELD

727.248

40.6

MOE-TELIS

PLOV IIPT

0.02 .3

0.04 .0

0.04 .0

0.0 4.0

$\begin{array}{ll}0.0 & 4.0 \\ 0.0 & 4.2\end{array}$

CONBTY : RARTLEY

.0

coogry

HABTL BI
GAS-PBD

$\begin{array}{ll}4328 & 5.1 \\ 8047 & 5\end{array}$

8047
16645

16645.91 .4
$44248 \quad 30.3$

82638 . 56.6

$49219 \quad 32$.

\section{4}

214

401

948

2791

5183

3050

7.27, 248 CUEOL ATIVE PROD. PROA ABANDOBEB FIELDS 
ABANDONED ONSHORE OIL FIELDS FITH OVER 250,000 BBL. CUMOLATIVE FROCUCT ION

DST DISC ABD. PLD NOM FIBLD NAEE

1019591966,70743071 PERRYTON (CLEVELAND)

DEPTH GRAVITI

6,688

40.8

Y E A R

1966

COONTY: OCHILTREE.
-

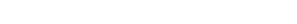

1 'PIELD (S)
COHOL. PROD. OF FIELD

323,243

COONTI

OĆH ILTREE 
ABANDONED ONSHOEE OIL PIELDS ITH OVER 250,000 BBL. CURULATIVE PRODOCTICN

DST DISC ABD. DLD NOM PIEID NAME

$10 \quad 1955 \quad 1970 \quad 73768852$ QTINDUNO (TORONTO)

$\begin{array}{cccccc}\text { DEPTH } & \text { GRAVITY } & \text { COMUL. } & \text { PROD. OP FIELD } & \text { CCONTY } \\ 6.274 & 40.2 & \vdots & 352,592 & \text { BOBERTS }\end{array}$

YE AR

NOL-RELLS. FLOR IIFT

1970

1969

1968

1967

1966

COONTY: ROBRRT

1 PIELD (S)

DISTRICT 10

$\begin{array}{rr}\text { OIL-PRD } & \text { E/RI } \\ \cdots & \\ 6385 & 11.7 \\ 13933 & 19.1 \\ 14515 & 19.9 \\ 8268 & 11.3 \\ 10478 & 14.4\end{array}$

1047814.4 $\begin{array}{rr}0.0 & 1.5 \\ 0.3 & 1.8\end{array}$

$1.0 \quad 1.0$

1.01 .0
ALL DISTRICTS

GA STRD

623

940
2167

55665

55665

352,592 COAOLATIVE PROD. PROM ABANDONBD FIELDS 1,403,083 COMOLATIVB PROD. PROM ABALCOMEL PIELDS

$760,684,612$ COSOLATIVE PROD. PROQ ABAMLONEL FIEIDS 